\title{
Pred-Skin: A web portal for accurate prediction of
}

\section{human skin sensitizers}

Joyce V. B. Borba, ${ }^{a, b, \neq}$ Rodolpho C. Braga, ${ }^{c, \neq}$ Vinicius M. Alves,, , Eugene N. Muratov, ${ }^{b, d}$ Nicole Kleinstreuer, ${ }^{e}$ Alexander Tropsha, ${ }^{b}$ and Carolina H. Andrade ${ }^{a, *}$

${ }^{a}$ Laboratory for Molecular Modeling and Drug Design, Faculty of Pharmacy, Universidade Federal de Goiás, Goiânia, GO, 74605-170, Brazil.

${ }^{b}$ Laboratory for Molecular Modeling, UNC Eshelman School of Pharmacy, the University of North Carolina at Chapel Hill, Chapel Hill, NC, 27599, USA.

c InsilicAll, Sao Paulo, SP, Brazil.

${ }^{d}$ Department of Pharmaceutical Sciences, Federal University of Paraiba, Joao Pessoa, PB, 58059, Brazil.

${ }^{e}$ National Toxicology Program Interagency Center for the Evaluation of Alternative Toxicological Methods, National Institute of Environmental Health Sciences, Research Triangle Park, NC, USA. 
Table S1. Skin sensitization data from all five assays

\begin{tabular}{|c|c|c|c|c|c|c|c|c|c|}
\hline Compound name & SMILES & \begin{tabular}{|l|}
$\begin{array}{l}\text { Human } \\
\text { result }\end{array}$ \\
\end{tabular} & $\begin{array}{l}\text { LLNA } \\
\text { result }\end{array}$ & DPRA & KeratinoSens & h-CLAT & $\begin{array}{l}\text { Human } \\
\text { reference }\end{array}$ & LLNA reference & $\begin{array}{l}\text { Non-animal } \\
\text { reference }\end{array}$ \\
\hline $\begin{array}{l}\text { 1,2-Benzisothiazolin-3- } \\
\text { one }\end{array}$ & $\mathrm{O}=\mathrm{C} 1 \mathrm{NSc} 2 \operatorname{cccc} 12$ & Sensitizer & Sensitizer & Positive & Positive & Positive & $\begin{array}{l}\text { ICCVAM (2011) } \\
\text { and Basketter et } \\
\text { al. (2014) }\end{array}$ & ICCVAM (2013) & Urbisch et al. (2015) \\
\hline $\begin{array}{l}\text { 1,2-Dibromo-2,4- } \\
\text { dicyanobutane }\end{array}$ & $\begin{array}{l}\mathrm{BrCC}(\mathrm{Br})(\mathrm{CCC} \# \mathrm{~N}) \mathrm{C} \# \\
\mathrm{~N}\end{array}$ & Sensitizer & Sensitizer & Positive & Positive & Positive & $\begin{array}{l}\text { Basketter et al. } \\
(2014)\end{array}$ & ICCVAM (2013) & Urbisch et al. (2015) \\
\hline 1,4-Dihydroquinone & Oc1ccc(O)cc1 & Sensitizer & Sensitizer & Positive & Positive & Positive & $\begin{array}{l}\text { Basketter et al. } \\
(2014)\end{array}$ & $\begin{array}{l}\text { ICCVAM }(2013)+ \\
\text { REACH }(2008-2014)\end{array}$ & Urbisch et al. (2015) \\
\hline $\begin{array}{l}2,4- \\
\text { Dinitrochlorobenzene }\end{array}$ & $\begin{array}{l}\text { Clc1ccc }(\operatorname{cc} 1 \mathrm{~N}(=\mathrm{O})=\mathrm{O}) \\
\mathrm{N}(=\mathrm{O})=\mathrm{O}\end{array}$ & Sensitizer & Sensitizer & Positive & Positive & Positive & $\begin{array}{l}\text { ICCVAM (2011) } \\
\text { and Basketter et } \\
\text { al. (2014) }\end{array}$ & ICCVAM (2013) & Urbisch et al. (2015) \\
\hline 2,5-Toluenediamine & $\operatorname{Cc} 1 \operatorname{cc}(\mathrm{N}) \operatorname{ccc} 1 \mathrm{~N}$ & Sensitizer & Sensitizer & Positive & Positive & Positive & $\begin{array}{l}\text { Basketter et al. } \\
\text { (2014) }\end{array}$ & $\begin{array}{l}\text { ICCVAM }(2013)+ \\
\text { REACH }(2008-2014)\end{array}$ & Urbisch et al. (2015) \\
\hline 2-Aminophenol & $\mathrm{Nc1} \operatorname{ccccc} 1 \mathrm{O}$ & Sensitizer & Sensitizer & Positive & Positive & Positive & $\begin{array}{l}\text { Basketter et al. } \\
(2014)\end{array}$ & ICCVAM (2013) & Urbisch et al. (2015) \\
\hline 2-Hydroxyethyl acrylate & $\mathrm{OCCOC}(=\mathrm{O}) \mathrm{C}=\mathrm{C}$ & Sensitizer & Sensitizer & Positive & Positive & Positive & $\begin{array}{l}\text { Basketter et al. } \\
(2014)\end{array}$ & ICCVAM (2013) & Urbisch et al. (2015) \\
\hline $\begin{array}{l}2- \\
\text { Mercaptobenzothiazole }\end{array}$ & $\mathrm{S}=\mathrm{C} 1 \mathrm{Nc} 2 \mathrm{ccccc} 2 \mathrm{~S} 1$ & Sensitizer & Sensitizer & Positive & Positive & Positive & $\begin{array}{l}\text { ICCVAM (2011) } \\
\text { and Basketter et } \\
\text { al. (2014) }\end{array}$ & ICCVAM (2013) & Urbisch et al. (2015) \\
\hline $\begin{array}{l}\text { 2-Nitro-1,4- } \\
\text { phenylenediamine }\end{array}$ & $\begin{array}{l}\mathrm{Nc} 1 \operatorname{ccc}(\mathrm{N}) \mathrm{c}(\mathrm{c} 1) \mathrm{N}(=\mathrm{O}) \\
=\mathrm{O}\end{array}$ & Sensitizer & Sensitizer & Positive & Positive & Positive & $\begin{array}{l}\text { Basketter et al. } \\
(2014)\end{array}$ & ICCVAM (2013) & Urbisch et al. (2015) \\
\hline $\begin{array}{l}3 \text { and 4-(4-Hydroxy-4- } \\
\text { methylpentyl)-3- } \\
\text { cyclohexane-1-carbox- } \\
\text { aldehyde [Lyral } \\
\text { HMPCC] }\end{array}$ & $\begin{array}{l}\mathrm{CC}(\mathrm{C})(\mathrm{O}) \mathrm{CCCC} 1=\mathrm{CC} \\
\mathrm{C}(\mathrm{CC} 1) \mathrm{C}=\mathrm{O}\end{array}$ & Sensitizer & Sensitizer & Positive & Positive & Positive & $\begin{array}{l}\text { Basketter et al. } \\
\text { (2014) }\end{array}$ & ICCVAM (2013) & Urbisch et al. (2015) \\
\hline $\begin{array}{l}3,3^{\prime}, 4^{\prime}, 5- \\
\text { Tetrachlorosalicylanilid } \\
\text { e }\end{array}$ & $\begin{array}{l}\text { Oc1c(Cl)cc(Cl)cc1C(= } \\
\text { O)Nc1 } 1 \mathrm{ccc}(\mathrm{Cl}) \mathrm{c}(\mathrm{Cl}) \mathrm{cl} 1\end{array}$ & Sensitizer & Sensitizer & Positive & Positive & Positive & $\begin{array}{l}\text { ICCVAM (2011) } \\
\text { and Basketter et } \\
\text { al. (2014) }\end{array}$ & ICCVAM (2013) & Urbisch et al. (2015) \\
\hline 4-Phenylenediamine & $\mathrm{Nc1} \operatorname{ccc}(\mathrm{N}) \mathrm{ccl}$ & Sensitizer & Sensitizer & Positive & Positive & Positive & $\begin{array}{l}\text { ICCVAM (2011) } \\
\text { and Basketter et } \\
\text { al. (2014) }\end{array}$ & $\begin{array}{l}\text { ICCVAM (2013) + } \\
\text { REACH }(2008-2014)\end{array}$ & Urbisch et al. (2015) \\
\hline $\begin{array}{l}\text { 5-Methyl-2,3- } \\
\text { hexanedione (acetyl } \\
\text { isovaleryl) }\end{array}$ & $\mathrm{CC}(\mathrm{C}) \mathrm{CC}(=\mathrm{O}) \mathrm{C}(\mathrm{O})=\mathrm{C}$ & Sensitizer & Sensitizer & Positive & Positive & Positive & $\begin{array}{l}\text { ICCVAM (2011) } \\
\text { and Basketter et } \\
\text { al. (2014) }\end{array}$ & ICCVAM (2013) & Urbisch et al. (2015) \\
\hline Bandrowski's base & $\begin{array}{l}\mathrm{Nc} 1 \mathrm{ccc}(\mathrm{cc} 1) \mathrm{N}=\mathrm{C} 1 \mathrm{CC}( \\
=\mathrm{N}) \mathrm{C}(\mathrm{CC} 1=\mathrm{N})=\mathrm{Nc} 1 \mathrm{cc} \\
\mathrm{c}(\mathrm{N}) \mathrm{cc} 1\end{array}$ & Sensitizer & Sensitizer & Positive & Positive & Positive & $\begin{array}{l}\text { White et al. } \\
(2006)\end{array}$ & ICCVAM (2013) & Urbisch et al. (2015) \\
\hline Benzyl bromide & BrCc1 $1 \mathrm{cccc} 1$ & Sensitizer & Sensitizer & Positive & Positive & Positive & $\begin{array}{l}\text { Basketter et al. } \\
\text { (1999) }\end{array}$ & ICCVAM (2013) & Urbisch et al. (2015) \\
\hline Benzylidene acetone & $\mathrm{CC}(=\mathrm{O}) \mathrm{C}=\mathrm{Cc} 1 \mathrm{ccccc} 1$ & Sensitizer & Sensitizer & Positive & Positive & Positive & ICCVAM (2011) & ICCVAM (2013) & Urbisch et al. (2015) \\
\hline
\end{tabular}




\begin{tabular}{|c|c|c|c|c|c|c|c|c|c|}
\hline Compound name & SMILES & $\begin{array}{l}\text { Human } \\
\text { result }\end{array}$ & $\begin{array}{l}\text { LLNA } \\
\text { result }\end{array}$ & DPRA & KeratinoSens & h-CLAT & Human reference & LLNA reference & Non-animal reference \\
\hline $\begin{array}{l}\text { Bisphenol A diglycidyl } \\
\text { ether }\end{array}$ & $\begin{array}{l}\mathrm{CC}(\mathrm{C})(\mathrm{c} 1 \mathrm{ccc}(\mathrm{OCC} 2 \mathrm{CO} 2) \\
\mathrm{cc} 1) \mathrm{c} 1 \mathrm{ccc}(\mathrm{OCC} 2 \mathrm{CO} 2) \mathrm{cc} \\
1\end{array}$ & Sensitizer & Sensitizer & Positive & Positive & Positive & $\begin{array}{l}\text { Basketter et al. } \\
\text { (2014) }\end{array}$ & ICCVAM (2013) & Urbisch et al. (2015) \\
\hline Carvone & $\begin{array}{l}\mathrm{CC}(=\mathrm{C}) \mathrm{C} 1 \mathrm{CC}=\mathrm{C}(\mathrm{C}) \mathrm{C}(=\mathrm{O}) \\
\mathrm{C} 1\end{array}$ & Sensitizer & Sensitizer & Positive & Positive & Positive & ICCVAM (2011) & $\begin{array}{l}\text { ICCVAM (2013) + } \\
\text { REACH (2008-2014) }\end{array}$ & Urbisch et al. (2015) \\
\hline Cinnamic aldehyde & $\mathrm{O}=\mathrm{CC}=\mathrm{Cc} 1 \mathrm{ccccc} 1$ & Sensitizer & Sensitizer & Positive & Positive & Positive & $\begin{array}{l}\text { ICCVAM (2011) } \\
\text { and Basketter et } \\
\text { al. (2014) }\end{array}$ & ICCVAM (2013) & Urbisch et al. (2015) \\
\hline Cinnamyl alcohol & $\mathrm{OCC}=\mathrm{Cc} 1 \mathrm{ccccc} 1$ & Sensitizer & Sensitizer & Positive & Positive & Positive & $\begin{array}{l}\text { ICCVAM (2011) } \\
\text { and Basketter et } \\
\text { al. (2014) }\end{array}$ & ICCVAM (2013) & Urbisch et al. (2015) \\
\hline Citral & $\mathrm{CC}(\mathrm{C})=\mathrm{CCCC}(\mathrm{C})=\mathrm{CC}=\mathrm{O}$ & Sensitizer & Sensitizer & Positive & Positive & Positive & $\begin{array}{l}\text { ICCVAM (2011) } \\
\text { and Basketter et } \\
\text { al. (2014) }\end{array}$ & ICCVAM (2013) & Urbisch et al. (2015) \\
\hline Diethyl maleate & $\begin{array}{l}\mathrm{CCOC}(=0) \mathrm{C}=\mathrm{CC}(=0) O C \\
\mathrm{C}\end{array}$ & Sensitizer & Sensitizer & Positive & Positive & Positive & $\begin{array}{l}\text { ICCVAM (2011) } \\
\text { and Basketter et } \\
\text { al. (2014) }\end{array}$ & ICCVAM (2013) & Urbisch et al. (2015) \\
\hline $\begin{array}{l}\text { Diphenylcyclopropenon } \\
\text { e }\end{array}$ & $\begin{array}{l}\mathrm{O}=\mathrm{C} 1 \mathrm{C}(=\mathrm{C} 1 \mathrm{c} 1 \mathrm{ccccc} 1) \mathrm{c} \\
1 \mathrm{ccccc} 1\end{array}$ & Sensitizer & Sensitizer & Positive & Positive & Positive & $\begin{array}{l}\text { Basketter et al. } \\
\text { (2014) }\end{array}$ & ICCVAM (2013) & Urbisch et al. (2015) \\
\hline $\begin{array}{l}\text { Ethylene glycol } \\
\text { dimethacrylate }\end{array}$ & $\begin{array}{l}\mathrm{CC}(=\mathrm{C}) \mathrm{C}(=\mathrm{O}) \mathrm{OCCOC}(= \\
\mathrm{O}) \mathrm{C}(\mathrm{C})=\mathrm{C}\end{array}$ & Sensitizer & Sensitizer & Positive & Positive & Positive & $\begin{array}{l}\text { Basketter et al. } \\
\text { (2014) }\end{array}$ & ICCVAM (2013) & Urbisch et al. (2015) \\
\hline Ethylenediamine & NCCN & Sensitizer & Sensitizer & Positive & Positive & Positive & $\begin{array}{l}\text { ICCVAM (2011) } \\
\text { and Basketter et } \\
\text { al. (2014) }\end{array}$ & ICCVAM (2013) & Urbisch et al. (2015) \\
\hline Formaldehyde & $\mathrm{C}=\mathrm{O}$ & Sensitizer & Sensitizer & Positive & Positive & Positive & $\begin{array}{l}\text { ICCVAM (2011) } \\
\text { and Basketter et } \\
\text { al. (2014) }\end{array}$ & $\begin{array}{l}\text { ICCVAM (2013) + } \\
\text { REACH }(2008-2014)\end{array}$ & Urbisch et al. (2015) \\
\hline Glutaraldehyde & $\mathrm{O}=\mathrm{CCCCC}=\mathrm{O}$ & Sensitizer & Sensitizer & Positive & Positive & Positive & $\begin{array}{l}\text { ICCVAM (2011) } \\
\text { and Basketter et } \\
\text { al. (2014) }\end{array}$ & ICCVAM (2013) & Urbisch et al. (2015) \\
\hline Glyoxal & $\mathrm{O}=\mathrm{CC}=\mathrm{O}$ & Sensitizer & Sensitizer & Positive & Positive & Positive & $\begin{array}{l}\text { ICCVAM (2011) } \\
\text { and Basketter et } \\
\text { al. (2014) }\end{array}$ & $\begin{array}{l}\text { ICCVAM }(2013)+ \\
\operatorname{REACH}(2008-2014)\end{array}$ & Urbisch et al. (2015) \\
\hline Hydroxycitronellal & $\mathrm{CC}(\mathrm{CCCC}(\mathrm{C})(\mathrm{C}) \mathrm{O}) \mathrm{CC}=\mathrm{O}$ & Sensitizer & Sensitizer & Positive & Positive & Positive & $\begin{array}{l}\text { ICCVAM (2011) } \\
\text { and Basketter et } \\
\text { al. (2014) }\end{array}$ & ICCVAM (2013) & Urbisch et al. (2015) \\
\hline Imidazolidinyl urea & $\begin{array}{l}\text { OCN1C(NC(=O)NCNC(= } \\
\text { O)NC2N(CO)C(=O)NC2 } \\
=0) C(=0) N C 1=0\end{array}$ & Sensitizer & Sensitizer & Positive & Positive & Positive & $\begin{array}{l}\text { ICCVAM (2011) } \\
\text { and Basketter et } \\
\text { al. (2014) }\end{array}$ & ICCVAM (2013) & Urbisch et al. (2015) \\
\hline Lauryl gallate & $\begin{array}{l}\operatorname{cccccccccccCOC}(=0 \\
\operatorname{cc} 1 \mathrm{cc}(\mathrm{O}) \mathrm{c}(\mathrm{O}) \mathrm{c}(\mathrm{O}) \mathrm{c} 1\end{array}$ & Sensitizer & Sensitizer & Positive & Positive & Positive & $\begin{array}{l}\text { Basketter et al. } \\
\text { (2014) }\end{array}$ & ICCVAM (2013) & Urbisch et al. (2015) \\
\hline Methyl 2-nonynoate & CCCCCCC\#CC $(=0) O C$ & Sensitizer & Sensitizer & Positive & Positive & Positive & ICCVAM (2011) & ICCVAM (2013) & Urbisch et al. (2015) \\
\hline Methyl 2-octynoate & CCCCCC\#CC $(=O) O C$ & Sensitizer & Sensitizer & Positive & Positive & Positive & ICCVAM (2011) & ICCVAM (2013) & Urbisch et al. (2015) \\
\hline
\end{tabular}




\begin{tabular}{|c|c|c|c|c|c|c|c|c|c|}
\hline Compound name & SMILES & $\begin{array}{l}\text { Human } \\
\text { result }\end{array}$ & $\begin{array}{l}\text { LLNA } \\
\text { result }\end{array}$ & DPRA & KeratinoSens & h-CLAT & Human reference & LLNA reference & Non-animal reference \\
\hline Methyl methacrylate & $\operatorname{coc}(=O) C(C)=C$ & Sensitizer & Sensitizer & Positive & Positive & Positive & $\begin{array}{l}\text { Basketter et al. } \\
(2014)\end{array}$ & $\begin{array}{l}\text { ICCVAM (2013) + } \\
\text { REACH (2008-2014) }\end{array}$ & Urbisch et al. (2015) \\
\hline Methylisothiazolinone & $\mathrm{CN} 1 \mathrm{SC}=\mathrm{CC} 1=\mathrm{O}$ & Sensitizer & Sensitizer & Positive & Positive & Positive & $\begin{array}{l}\text { ICCVAM (2011) } \\
\text { and Basketter et } \\
\text { al. (2014) }\end{array}$ & ICCVAM (2013) & Urbisch et al. (2015) \\
\hline Oxazolone & $\begin{array}{l}\mathrm{CCOC}=\mathrm{C} 1 \mathrm{~N}=\mathrm{C}(\mathrm{OC} 1=0) \\
\mathrm{c} 1 \mathrm{ccccc} 1\end{array}$ & Sensitizer & Sensitizer & Positive & Positive & Positive & $\begin{array}{l}\text { Basketter and } \\
\text { Kimber (2006) }\end{array}$ & ICCVAM (2013) & Urbisch et al. (2015) \\
\hline Perillaldehyde & $\begin{array}{l}\mathrm{CC}(=\mathrm{C}) \mathrm{C} 1 \mathrm{CCC}(\mathrm{C}=0)=\mathrm{CC} \\
1\end{array}$ & Sensitizer & Sensitizer & Positive & Positive & Positive & ICCVAM (2011) & ICCVAM (2013) & Urbisch et al. (2015) \\
\hline Phenylacetaldehyde & $\mathrm{O}=\mathrm{CCc} 1 \mathrm{ccccc} 1$ & Sensitizer & Sensitizer & Positive & Positive & Positive & ICCVAM (2011) & ICCVAM (2013) & Urbisch et al. (2015) \\
\hline Propyl gallate & $\begin{array}{l}\mathrm{cCCOC}(=0) \mathrm{c} 1 \mathrm{cc}(\mathrm{O}) \mathrm{c}(\mathrm{O}) \\
\mathrm{c}(\mathrm{O}) \mathrm{c} 1\end{array}$ & Sensitizer & Sensitizer & Positive & Positive & Positive & $\begin{array}{l}\text { Basketter et al. } \\
\text { (2014) }\end{array}$ & ICCVAM (2013) & Urbisch et al. (2015) \\
\hline $\begin{array}{l}\text { Tetramethylthiuram } \\
\text { disulfide }\end{array}$ & $\begin{array}{l}\mathrm{CN}(\mathrm{C}) \mathrm{C}(=\mathrm{S}) \mathrm{SSC}(=\mathrm{S}) \mathrm{N}(\mathrm{C}) \\
\mathrm{C}\end{array}$ & Sensitizer & Sensitizer & Positive & Positive & Positive & $\begin{array}{l}\text { ICCVAM (2011) } \\
\text { and Basketter et } \\
\text { al. (2014) }\end{array}$ & ICCVAM (2013) & Urbisch et al. (2015) \\
\hline p-Benzoquinone & $\mathrm{O}=\mathrm{C} 1 \mathrm{C}=\mathrm{CC}(=\mathrm{O}) \mathrm{C}=\mathrm{C} 1$ & Sensitizer & Sensitizer & Positive & Positive & Positive & $\begin{array}{l}\text { Basketter et al. } \\
\text { (1999) }\end{array}$ & ICCVAM (2013) & Urbisch et al. (2015) \\
\hline trans-2-Hexenal & $\mathrm{CCCC}=\mathrm{CC}=\mathrm{O}$ & Sensitizer & Sensitizer & Positive & Positive & Positive & ICCVAM (2011) & ICCVAM (2013) & Urbisch et al. (2015) \\
\hline Butyl glycidyl ether & cCCCOCC1CO1 & Sensitizer & Sensitizer & Positive & Positive & Negative & $\begin{array}{l}\text { ICCVAM (2011) } \\
\text { and Basketter et } \\
\text { al. (2014) }\end{array}$ & ICCVAM (2013) & Urbisch et al. (2015) \\
\hline Isoeugenol & $\operatorname{coc} 1 \mathrm{cc}(\mathrm{C}=\mathrm{CC}) \operatorname{ccc} 10$ & Sensitizer & Sensitizer & Positive & Positive & Negative & $\begin{array}{l}\text { ICCVAM (2011) } \\
\text { and Basketter et } \\
\text { al. (2014) }\end{array}$ & ICCVAM (2013) & Urbisch et al. (2015) \\
\hline Ethyl acrylate & $\operatorname{ccOC}(=\mathrm{O}) \mathrm{C}=\mathrm{C}$ & Sensitizer & Sensitizer & Positive & Positive & & ICCVAM (2011) & $\begin{array}{l}\text { ICCVAM (2013) + } \\
\text { REACH }(2008-2014)\end{array}$ & Urbisch et al. (2015) \\
\hline $\begin{array}{l}\text { Methylanisylidene } \\
\text { acetone }\end{array}$ & $\begin{array}{l}\mathrm{CCC}(=0) \mathrm{C}=\mathrm{Cc} 1 \mathrm{ccc}(\mathrm{OC}) \\
\mathrm{cc} 1\end{array}$ & Sensitizer & Sensitizer & Positive & Positive & & ICCVAM (2011) & ICCVAM (2013) & Urbisch et al. (2015) \\
\hline 3,4-Dihydrocoumarin & $\mathrm{O}=\mathrm{C} 1 \mathrm{CCc} 2 \operatorname{ccccc} 2 \mathrm{O} 1$ & Sensitizer & Sensitizer & Positive & Negative & Positive & ICCVAM (2011) & ICCVAM (2013) & Urbisch et al. (2015) \\
\hline 3-Propylidenephthalide & $\begin{array}{l}\mathrm{CCC}=\mathrm{C} 1 \mathrm{OC}(=0) \mathrm{c} 2 \mathrm{ccccc} \\
12\end{array}$ & Sensitizer & Sensitizer & Positive & Negative & Positive & ICCVAM (2011) & ICCVAM (2013) & Urbisch et al. (2015) \\
\hline Citronellol & $\mathrm{CC}(\mathrm{CCO}) \mathrm{CCC}=\mathrm{C}(\mathrm{C}) \mathrm{C}$ & Sensitizer & Sensitizer & Positive & Negative & Positive & ICCVAM (2011) & ICCVAM (2013) & Urbisch et al. (2015) \\
\hline Maleic anhydride & $\mathrm{O}=\mathrm{C} 1 \mathrm{OC}(=\mathrm{O}) \mathrm{C}=\mathrm{C} 1$ & Sensitizer & Sensitizer & Positive & Negative & Positive & $\begin{array}{l}\text { Basketter and } \\
\text { Kimber (2006) }\end{array}$ & REACH (2008-2014) & Urbisch et al. (2015) \\
\hline Penicillin G & $\begin{array}{l}\mathrm{CC} 1(\mathrm{C}) \mathrm{SC} 2 \mathrm{C}(\mathrm{NC}(=\mathrm{O}) \mathrm{Cc} \\
3 \operatorname{ccccc} 3) \mathrm{C}(=\mathrm{O}) \mathrm{N} 2 \mathrm{C} 1 \mathrm{C}( \\
\mathrm{O})=0\end{array}$ & Sensitizer & Sensitizer & Positive & Negative & Positive & $\begin{array}{l}\text { ICCVAM (2011) } \\
\text { and Basketter et } \\
\text { al. (2014) }\end{array}$ & ICCVAM (2013) & Urbisch et al. (2015) \\
\hline Phenyl benzoate & $\begin{array}{l}\mathrm{O}=\mathrm{C}(\mathrm{O} 1 \mathrm{ccccc} 1) \mathrm{c} 1 \mathrm{cccc} \\
\mathrm{c} 1\end{array}$ & Sensitizer & Sensitizer & Positive & Negative & Positive & $\begin{array}{l}\text { ICCVAM (2011) } \\
\text { and Basketter et } \\
\text { al. (2014) }\end{array}$ & ICCVAM (2013) & Urbisch et al. (2015) \\
\hline
\end{tabular}




\begin{tabular}{|c|c|c|c|c|c|c|c|c|c|}
\hline Compound name & SMILES & $\begin{array}{l}\text { Human } \\
\text { result }\end{array}$ & $\begin{array}{l}\text { LLNA } \\
\text { result }\end{array}$ & DPRA & KeratinoSens & h-CLAT & $\begin{array}{l}\text { Human } \\
\text { reference }\end{array}$ & LLNA reference & Non-animal reference \\
\hline Trimellitic anhydride & $\begin{array}{l}\mathrm{OC}(=0) \mathrm{c} 1 \mathrm{ccc} 2 \mathrm{C}(=0) \mathrm{OC} \\
(=0) \mathrm{c} 2 \mathrm{c} 1\end{array}$ & Sensitizer & Sensitizer & Positive & Negative & Positive & $\begin{array}{l}\text { Basketter and } \\
\text { Kimber (2006) }\end{array}$ & ICCVAM (2013) & Urbisch et al. (2015) \\
\hline $\begin{array}{l}\text { p-tert-Butyl- } \alpha- \\
\text { methylhydrocinnamic } \\
\text { aldehyde (Lilial) }\end{array}$ & $\begin{array}{l}\mathrm{CC}(\mathrm{Cc} 1 \mathrm{ccc}(\mathrm{cc} 1) \mathrm{C}(\mathrm{C})(\mathrm{C}) \\
\mathrm{C}) \mathrm{C}=\mathrm{O}\end{array}$ & Sensitizer & Sensitizer & Positive & Negative & Positive & $\begin{array}{l}\text { ICCVAM (2011) } \\
\text { and Basketter et } \\
\text { al. (2014) }\end{array}$ & ICCVAM (2013) & Urbisch et al. (2015) \\
\hline Benzoyl peroxide & $\begin{array}{l}\mathrm{O}=\mathrm{C}(\mathrm{OOC}(=0) \mathrm{c} 1 \mathrm{ccccc} 1 \\
) \mathrm{c} 1 \mathrm{ccccc} 1\end{array}$ & Sensitizer & Sensitizer & Positive & Negative & Negative & $\begin{array}{l}\text { ICCVAM (2011) } \\
\text { and Basketter et } \\
\text { al. (2014) }\end{array}$ & $\begin{array}{l}\text { ICCVAM }(2013)+ \\
\text { REACH }(2008-2014)\end{array}$ & Urbisch et al. (2015) \\
\hline Phthalic anhydride & $\mathrm{O}=\mathrm{C} 10 \mathrm{C}(=0) \operatorname{cocccc} 12$ & Sensitizer & Sensitizer & Positive & Negative & Negative & $\begin{array}{l}\text { Basketter and } \\
\text { Kimber (2006) }\end{array}$ & $\begin{array}{l}\text { REACH (2008-2014) + J } \\
\text { Appl Toxicol, v 33(11), } \\
\text { 1353-64, } 2013 .\end{array}$ & Urbisch et al. (2015) \\
\hline Eugenol & $\operatorname{coc} 1 \mathrm{cc}(\mathrm{CC}=\mathrm{C}) \operatorname{ccc} 10$ & Sensitizer & Sensitizer & Positive & & Positive & $\begin{array}{l}\text { ICCVAM (2011) } \\
\text { and Basketter et } \\
\text { al. (2014) }\end{array}$ & $\begin{array}{l}\text { ICCVAM (2013) + } \\
\text { REACH }(2008-2014)\end{array}$ & Urbisch et al. (2015) \\
\hline $\begin{array}{l}\text { 3- } \\
\text { Dimethylaminopropyla } \\
\text { mine }\end{array}$ & $\mathrm{CN}(\mathrm{C}) \mathrm{CCCN}$ & Sensitizer & Sensitizer & Negative & Positive & Positive & $\begin{array}{l}\text { Basketter et al. } \\
\text { (2014) }\end{array}$ & $\begin{array}{l}\operatorname{ICCVAM}(2013)+ \\
\operatorname{REACH}(2008-2014)\end{array}$ & Urbisch et al. (2015) \\
\hline Aniline & Nc1ccccc1 & Sensitizer & Sensitizer & Negative & Positive & Positive & $\begin{array}{l}\text { ICCVAM (2011) } \\
\text { and Basketter et } \\
\text { al. (2014) }\end{array}$ & $\begin{array}{l}\text { ICCVAM }(2013)+ \\
\text { REACH }(2008-2014)\end{array}$ & Urbisch et al. (2015) \\
\hline Geraniol & $\mathrm{CC}(\mathrm{C})=\mathrm{CCCC}(\mathrm{C})=\mathrm{CCO}$ & Sensitizer & Sensitizer & Negative & Positive & Positive & $\begin{array}{l}\text { ICCVAM (2011) } \\
\text { and Basketter et } \\
\text { al. (2014) }\end{array}$ & ICCVAM (2013) & Urbisch et al. (2015) \\
\hline $\begin{array}{l}\alpha \text {-Amyl cinnamic } \\
\text { aldehyde }\end{array}$ & $\begin{array}{l}\operatorname{cccccC}(C=0)=\operatorname{Cc} 1 \operatorname{cccc} \\
\mathrm{c} 1\end{array}$ & Sensitizer & Sensitizer & Negative & Positive & Positive & $\begin{array}{l}\text { Basketter et al. } \\
(2014)\end{array}$ & $\begin{array}{l}\text { ICCVAM }(2013)+ \\
\text { REACH }(2008-2014)\end{array}$ & Urbisch et al. (2015) \\
\hline 3-Aminophenol & Nc1cccc(O)c1 & Sensitizer & Sensitizer & Negative & Negative & Positive & $\begin{array}{l}\text { Basketter et al. } \\
\text { (1999) }\end{array}$ & $\begin{array}{l}\text { ICCVAM }(2013)+ \\
\text { REACH }(2008-2014)\end{array}$ & Urbisch et al. (2015) \\
\hline Resorcinol & Oc1cccc(O)c1 & Sensitizer & Sensitizer & Negative & Negative & Positive & $\begin{array}{l}\text { Basketter et al. } \\
\text { (2014) }\end{array}$ & $\begin{array}{l}\text { ICCVAM }(2013)+ \\
\operatorname{REACH}(2008-2014)\end{array}$ & Urbisch et al. (2015) \\
\hline Diethylenetriamine & NCCNCCN & Sensitizer & Sensitizer & Negative & Negative & Negative & ICCVAM (2011) & $\begin{array}{l}\text { ICCVAM }(2013)+ \\
\text { REACH }(2008-2014)\end{array}$ & Urbisch et al. (2015) \\
\hline Thioglycerol & $\mathrm{OCC}(\mathrm{O}) \mathrm{CS}$ & Sensitizer & Sensitizer & & & & ICCVAM (2011) & ICCVAM (2013) & \\
\hline $\begin{array}{l}\text { p-Methylhydrocinnamic } \\
\text { aldehyde }\end{array}$ & $\operatorname{Cc} 1 \mathrm{ccc}(\mathrm{CCC}=0) \operatorname{cc} 1$ & Sensitizer & Sensitizer & & & & ICCVAM (2011) & ICCVAM (2013) & \\
\hline Benzocaine & $\operatorname{cCOC}(=0) \operatorname{coccc}(\mathrm{N}) \operatorname{cc} 1$ & Sensitizer & $\begin{array}{l}\text { Non- } \\
\text { sensitizer }\end{array}$ & Positive & Positive & Positive & $\begin{array}{l}\text { ICCVAM (2011) } \\
\text { and Basketter et } \\
\text { al. (2014) }\end{array}$ & ICCVAM (2013) & Urbisch et al. (2015) \\
\hline $\begin{array}{l}\text { 2-Hydroxypropyl } \\
\text { methacrylate }\end{array}$ & $\mathrm{CC}(\mathrm{O}) \mathrm{COC}(=\mathrm{O}) \mathrm{C}(\mathrm{C})=\mathrm{C}$ & Sensitizer & $\begin{array}{l}\text { Non- } \\
\text { sensitizer }\end{array}$ & Positive & Positive & Negative & Bjorkner (1984) & ICCVAM (2013) & Urbisch et al. (2015) \\
\hline Abietic acid & $\begin{array}{l}\mathrm{CC}(\mathrm{C}) \mathrm{C} 1=\mathrm{CC} 2=\mathrm{CCC} 3 \mathrm{C}(\mathrm{C} \\
)(\mathrm{CCCC} 3(\mathrm{C}) \mathrm{C}(\mathrm{O})=0) \mathrm{C} 2 \mathrm{C} \\
\mathrm{C} 1\end{array}$ & Sensitizer & $\begin{array}{l}\text { Non- } \\
\text { sensitizer }\end{array}$ & Positive & Positive & Negative & $\begin{array}{l}\text { Basketter et al. } \\
\text { (2014) }\end{array}$ & $\begin{array}{l}\text { ICCVAM }(2013)+ \\
\operatorname{REACH}(2008-2014)\end{array}$ & Urbisch et al. (2015) \\
\hline
\end{tabular}




\begin{tabular}{|c|c|c|c|c|c|c|c|c|c|}
\hline Compound name & SMILES & $\begin{array}{l}\text { Human } \\
\text { result }\end{array}$ & $\begin{array}{l}\text { LLNA } \\
\text { result }\end{array}$ & DPRA & KeratinoSens & h-CLAT & $\begin{array}{l}\text { Human } \\
\text { reference }\end{array}$ & LLNA reference & Non-animal reference \\
\hline Coumarin & $\mathrm{O}=\mathrm{C} 1 \mathrm{Oc} 2 \operatorname{ccccc} 2 \mathrm{C}=\mathrm{C} 1$ & Sensitizer & $\begin{array}{l}\text { Non- } \\
\text { sensitizer }\end{array}$ & Negative & Positive & Negative & $\begin{array}{l}\text { ICCVAM (2011) } \\
\text { and Basketter et } \\
\text { al. (2014) }\end{array}$ & ICCVAM (2013) & Urbisch et al. (2015) \\
\hline Benzyl alcohol & OCc1ccccc1 & Sensitizer & $\begin{array}{l}\text { Non- } \\
\text { sensitizer }\end{array}$ & Negative & Negative & Positive & ICCVAM (2011) & ICCVAM (2013) & Urbisch et al. (2015) \\
\hline Linalool & $\operatorname{CC}(\mathrm{C})=\operatorname{CCCC}(\mathrm{C})(\mathrm{O}) \mathrm{C}=\mathrm{C}$ & Sensitizer & $\begin{array}{l}\text { Non- } \\
\text { sensitizer }\end{array}$ & Negative & Negative & Positive & $\begin{array}{l}\text { Basketter et al. } \\
(2014)\end{array}$ & $\begin{array}{l}\text { ICCVAM (2013) + } \\
\operatorname{REACH}(2008-2014)\end{array}$ & Urbisch et al. (2015) \\
\hline Streptomycin & $\begin{array}{l}\text { CNC1C(O)C(O)C(CO)OC } \\
10 C 1 C(O C 2 C(O) C(O) C( \\
N C(N)=N) C(O) C 2 N C(N) \\
=N) O C(C) C 1(O) C=O\end{array}$ & Sensitizer & $\begin{array}{l}\text { Non- } \\
\text { sensitizer }\end{array}$ & Negative & Negative & Negative & ICCVAM (2011) & ICCVAM (2013) & Urbisch et al. (2015) \\
\hline Sulfanilamide & $\begin{array}{l}\operatorname{Nc1} 1 \operatorname{ccc}(\operatorname{cc} 1) S(N)(=0)= \\
0\end{array}$ & Sensitizer & $\begin{array}{l}\text { Non- } \\
\text { sensitizer }\end{array}$ & Negative & Negative & Negative & ICCVAM (2011) & ICCVAM (2013) & Urbisch et al. (2015) \\
\hline Kanamycin & $\begin{array}{l}\text { NCC1OC(OC2C(N)CC(N } \\
\text { IC(OC3OC(CO)C(O)C(N } \\
\text { C3O)C2O)C(O)C(O)C1 } \\
O\end{array}$ & Sensitizer & $\begin{array}{l}\text { Non- } \\
\text { sensitizer }\end{array}$ & & Negative & Negative & ICCVAM (2011) & ICCVAM (2013) & Urbisch et al. (2015) \\
\hline Isocyclogeraniol & $\mathrm{CC} 1 \mathrm{CC}(\mathrm{C})=\mathrm{CC}(\mathrm{C}) \mathrm{C} 1 \mathrm{CO}$ & Sensitizer & $\begin{array}{l}\text { Non- } \\
\text { sensitizer }\end{array}$ & & & & ICCVAM (2011) & ICCVAM (2013) & \\
\hline Neomycin & $\begin{array}{l}\text { NCC1OC(OC2C(CO)OCl } \\
\text { OC3C(O)C(N)CC(N)C3O } \\
\text { C3OC(CN)C(O)C(O)C3N } \\
\text { C2O)C(N)C(O)C1O }\end{array}$ & Sensitizer & $\begin{array}{l}\text { Non- } \\
\text { sensitizer }\end{array}$ & & & & ICCVAM (2011) & ICCVAM (2013) & \\
\hline $\begin{array}{l}\text { Pentaerythritol } \\
\text { triacrylate }\end{array}$ & $\begin{array}{l}\text { OCC }(\operatorname{COC}(=O) C=C)(C O \\
C(=O) C=C) \operatorname{COC}(=O) C=C\end{array}$ & Sensitizer & $\begin{array}{l}\text { Non- } \\
\text { sensitizer }\end{array}$ & & & & ICCVAM (2011) & ICCVAM (2013) & \\
\hline$\alpha$-Amylcinnamyl alcohol & $\begin{array}{l}\operatorname{cccccC}(\mathrm{CO})=\operatorname{Cc} 1 \operatorname{ccccc} \\
1\end{array}$ & Sensitizer & $\begin{array}{l}\text { Non- } \\
\text { sensitizer }\end{array}$ & & & & ICCVAM (2011) & ICCVAM (2013) & \\
\hline Farnesol & $\begin{array}{l}\mathrm{CC}(\mathrm{C})=\mathrm{CCCC}(\mathrm{C})=\mathrm{CCCC}( \\
\mathrm{C})=\mathrm{CCO}\end{array}$ & Sensitizer & & Negative & Positive & Positive & ICCVAM (2011) & & Urbisch et al. (2015) \\
\hline 1-Octen-3-yl acetate & $\operatorname{CccccC}(O C(C)=O) C=C$ & Sensitizer & & & & & ICCVAM (2011) & & \\
\hline $\begin{array}{l}\text { 2-Hexylidene } \\
\text { cyclopentanone }\end{array}$ & $\mathrm{CCCCCC}=\mathrm{C} 1 \mathrm{CCCC} 1=\mathrm{O}$ & Sensitizer & & & & & ICCVAM (2011) & & \\
\hline Bourgeonal & $\begin{array}{l}\mathrm{CC}(\mathrm{C})(\mathrm{C}) \operatorname{c1ccc}(\mathrm{CCC}=0) \mathrm{c} \\
\mathrm{c} 1\end{array}$ & Sensitizer & & & & & ICCVAM (2011) & & \\
\hline Chlorpromazine & $\begin{array}{l}\mathrm{CN}(\mathrm{C}) \mathrm{CCCN} 1 \mathrm{c} 2 \mathrm{ccccc} 2 \mathrm{~S} \\
\mathrm{c} 2 \mathrm{ccc}(\mathrm{Cl}) \mathrm{cc} 12\end{array}$ & Sensitizer & & & & & ICCVAM (2011) & & \\
\hline Cinnamyl nitrile & $\mathrm{N \# CC}=\mathrm{Cc} 1 \mathrm{ccccc} 1$ & Sensitizer & & & & & ICCVAM (2011) & & \\
\hline $\begin{array}{l}\text { Menthadiene-7-methyl } \\
\text { formate }\end{array}$ & $\begin{array}{l}\mathrm{CC}(\mathrm{C}) \mathrm{C} 1=\mathrm{CCC}(\mathrm{CCOC}=0) \\
=\mathrm{CC} 1\end{array}$ & Sensitizer & & & & & ICCVAM (2011) & & \\
\hline Phenylpropionaldehyde & $\mathrm{O}=\mathrm{CCC} 1 \mathrm{ccccc} 1$ & Sensitizer & & & & & ICCVAM (2011) & & \\
\hline delta Damascone & $\begin{array}{l}\mathrm{CC}=\mathrm{CC}(=0) \mathrm{C} 1 \mathrm{C}(\mathrm{C}) \mathrm{C}=\mathrm{CC} \\
\mathrm{C} 1(\mathrm{C}) \mathrm{C}\end{array}$ & Sensitizer & & & & & ICCVAM (2011) & & \\
\hline
\end{tabular}




\begin{tabular}{|c|c|c|c|c|c|c|c|c|c|}
\hline Compound name & SMILES & $\begin{array}{l}\text { Human } \\
\text { result }\end{array}$ & $\begin{array}{l}\text { LLNA } \\
\text { result }\end{array}$ & DPRA & KeratinoSens & h-CLAT & $\begin{array}{l}\text { Human } \\
\text { reference }\end{array}$ & LLNA reference & Non-animal reference \\
\hline $\begin{array}{l}\alpha \text {-Methyl cinnamic } \\
\text { aldehyde }\end{array}$ & $\mathrm{CC}(\mathrm{C}=\mathrm{O})=\mathrm{Cc} 1 \operatorname{ccccc} 1$ & $\begin{array}{l}\text { Non- } \\
\text { sensitizer }\end{array}$ & Sensitizer & Positive & Positive & Positive & ICCVAM (2011) & ICCVAM (2013) & Urbisch et al. (2015) \\
\hline Cyclamen aldehyde & $\begin{array}{l}\mathrm{CC}(\operatorname{Cc} 1 \mathrm{ccc}(\mathrm{cc} 1) \mathrm{C}(\mathrm{C}) \mathrm{C}) \mathrm{C} \\
=0\end{array}$ & $\begin{array}{l}\text { Non- } \\
\text { sensitizer }\end{array}$ & Sensitizer & Positive & Positive & Negative & ICCVAM (2011) & $\begin{array}{l}\text { ICCVAM (2013) + } \\
\text { REACH }(2008-2014)\end{array}$ & Urbisch et al. (2015) \\
\hline Pentachlorophenol & $\begin{array}{l}\text { Oc1c(Cl)c(Cl)c(Cl)c(Cl)c } \\
1 \mathrm{Cl}\end{array}$ & $\begin{array}{l}\text { Non- } \\
\text { sensitizer }\end{array}$ & Sensitizer & Positive & Negative & Positive & $\begin{array}{l}\text { Basketter et al. } \\
\text { (2014) }\end{array}$ & ICCVAM (2013) & Urbisch et al. (2015) \\
\hline R-Limonene & $\mathrm{CC}(=\mathrm{C}) \mathrm{C} 1 \mathrm{CCC}(\mathrm{C})=\mathrm{CC} 1$ & $\begin{array}{l}\text { Non- } \\
\text { sensitizer }\end{array}$ & Sensitizer & Positive & Negative & Positive & ICCVAM (2011) & $\begin{array}{l}\text { ICCVAM }(2013)+ \\
\text { REACH }(2008-2014)\end{array}$ & Urbisch et al. (2015) \\
\hline Benzyl benzoate & $\begin{array}{l}\mathrm{O}=\mathrm{C}(\mathrm{OCc} 1 \mathrm{ccccc} 1) \mathrm{c} 1 \mathrm{ccc} \\
\mathrm{cc} 1\end{array}$ & $\begin{array}{l}\text { Non- } \\
\text { sensitizer }\end{array}$ & Sensitizer & Negative & Positive & Negative & $\begin{array}{l}\text { ICCVAM (2011) } \\
\text { and Basketter et } \\
\text { al. (2014) }\end{array}$ & $\begin{array}{l}\text { ICCVAM }(2013)+ \\
\text { REACH }(2008-2014)\end{array}$ & Urbisch et al. (2015) \\
\hline Benzyl cinnamate & $\begin{array}{l}\mathrm{O}=\mathrm{C}(\mathrm{O}(\mathrm{c} 1 \mathrm{cccc} c 1) \mathrm{C}=\mathrm{Cc} \\
1 \mathrm{ccccc} 1\end{array}$ & $\begin{array}{l}\text { Non- } \\
\text { sensitizer }\end{array}$ & Sensitizer & Negative & Positive & Negative & ICCVAM (2011) & ICCVAM (2013) & Urbisch et al. (2015) \\
\hline Benzyl salicylate & $\begin{array}{l}\text { Oc1ccccc1C(=0)OCc1c } \\
\text { cccc1 }\end{array}$ & $\begin{array}{l}\text { Non- } \\
\text { sensitizer }\end{array}$ & Sensitizer & Negative & Positive & Negative & ICCVAM (2011) & $\begin{array}{l}\text { ICCVAM }(2013)+ \\
\text { REACH }(2008-2014)\end{array}$ & Urbisch et al. (2015) \\
\hline $\begin{array}{l}\text { Hexyl cinnamic } \\
\text { aldehyde }\end{array}$ & $\begin{array}{l}\operatorname{ccccccc}(\mathrm{C}=0)=\operatorname{cc} 1 \mathrm{ccc} \\
\operatorname{cc1}\end{array}$ & $\begin{array}{l}\text { Non- } \\
\text { sensitizer }\end{array}$ & Sensitizer & Negative & Positive & Negative & $\begin{array}{l}\text { ICCVAM (2011) } \\
\text { and Basketter et } \\
\text { al. (2014) }\end{array}$ & $\begin{array}{l}\text { ICCVAM }(2013)+ \\
\text { REACH }(2008-2014)\end{array}$ & Urbisch et al. (2015) \\
\hline Tartaric acid & $\begin{array}{l}\mathrm{OC}(\mathrm{C}(\mathrm{O}) \mathrm{C}(\mathrm{O})=\mathrm{O}) \mathrm{C}(\mathrm{O})= \\
\mathrm{O}\end{array}$ & $\begin{array}{l}\text { Non- } \\
\text { sensitizer }\end{array}$ & Sensitizer & Negative & Positive & & ICCVAM (2011) & ICCVAM (2013) & Urbisch et al. (2015) \\
\hline $\begin{array}{l}\text { 2-Methoxy-4- } \\
\text { methylphenol }\end{array}$ & $\operatorname{coc} 1 \mathrm{cc}(\mathrm{C}) \operatorname{ccc} 10$ & $\begin{array}{l}\text { Non- } \\
\text { sensitizer }\end{array}$ & Sensitizer & Negative & Negative & Positive & ICCVAM (2011) & ICCVAM (2013) & Urbisch et al. (2015) \\
\hline Hexyl salicylate & $\begin{array}{l}\operatorname{ccccccOC}(=0) \operatorname{c} 1 \mathrm{ccccc} \\
10\end{array}$ & $\begin{array}{l}\text { Non- } \\
\text { sensitizer }\end{array}$ & Sensitizer & Negative & Negative & Positive & ICCVAM (2011) & $\begin{array}{l}\text { ICCVAM }(2013)+ \\
\operatorname{REACH}(2008-2014)\end{array}$ & Urbisch et al. (2015) \\
\hline Isomethyl- $\alpha$-ionone & $\begin{array}{l}\mathrm{CC}(=0) \mathrm{C}(\mathrm{C})=\mathrm{CC} 1 \mathrm{C}(\mathrm{C})=\mathrm{C} \\
\mathrm{CCC} 1(\mathrm{C}) \mathrm{C}\end{array}$ & $\begin{array}{l}\text { Non- } \\
\text { sensitizer }\end{array}$ & Sensitizer & Negative & Negative & Positive & ICCVAM (2011) & ICCVAM (2013) & Urbisch et al. (2015) \\
\hline Pyridine & c1ccncc1 & $\begin{array}{l}\text { Non- } \\
\text { sensitizer }\end{array}$ & Sensitizer & Negative & Negative & Positive & $\begin{array}{l}\text { Basketter et al. } \\
\text { (2014) }\end{array}$ & $\begin{array}{l}\text { ICCVAM }(2013)+ \\
\text { REACH }(2008-2014)\end{array}$ & Urbisch et al. (2015) \\
\hline Benzalkonium chloride & $\begin{array}{l}\text { ccCCCCCCCCCCCC[N+]( } \\
\text { C)(C) } \operatorname{cc} 1 \operatorname{ccccc} 1\end{array}$ & $\begin{array}{l}\text { Non- } \\
\text { sensitizer }\end{array}$ & Sensitizer & Negative & Negative & Negative & $\begin{array}{l}\text { ICCVAM (2011) } \\
\text { and Basketter et } \\
\text { al. (2014) }\end{array}$ & ICCVAM (2013) & Urbisch et al. (2015) \\
\hline Isopropyl myristate & $\begin{array}{l}\text { CCCCCCCCCCCCCCC }(=0) \\
\text { OC(C)C }\end{array}$ & $\begin{array}{l}\text { Non- } \\
\text { sensitizer }\end{array}$ & Sensitizer & Negative & Negative & & ICCVAM (2011) & ICCVAM (2013) & Urbisch et al. (2015) \\
\hline Sodium lauryl sulfate & $\begin{array}{l}\operatorname{cccccccccccccos}(0)( \\
=0)=0\end{array}$ & $\begin{array}{l}\text { Non- } \\
\text { sensitizer }\end{array}$ & Sensitizer & & Negative & Negative & $\begin{array}{l}\text { ICCVAM (2011) } \\
\text { and Basketter et } \\
\text { al. (2014) }\end{array}$ & ICCVAM (2013) & Urbisch et al. (2015) \\
\hline Anisyl alcohol & $\operatorname{cOc} 1 \mathrm{ccc}(\mathrm{CO}) \mathrm{cc} 1$ & $\begin{array}{l}\text { Non- } \\
\text { sensitizer }\end{array}$ & Sensitizer & & & & ICCVAM (2011) & ICCVAM (2013) & \\
\hline Isocyclemone E & $\begin{array}{l}\mathrm{CC} 1 \mathrm{CC} 2=\mathrm{C}(\mathrm{CC} 1(\mathrm{C}) \mathrm{C}(\mathrm{C})= \\
\mathrm{O}) \mathrm{C}(\mathrm{C})(\mathrm{C}) \mathrm{CCC} 2\end{array}$ & $\begin{array}{l}\text { Non- } \\
\text { sensitizer }\end{array}$ & Sensitizer & & & & ICCVAM (2011) & REACH (2008-2014) & \\
\hline Vanillin & $\operatorname{coc} 1 \mathrm{cc}(\mathrm{C}=0) \operatorname{ccc} 10$ & $\begin{array}{l}\text { Non- } \\
\text { sensitizer }\end{array}$ & $\begin{array}{l}\text { Non- } \\
\text { sensitizer }\end{array}$ & Positive & Positive & Negative & $\begin{array}{l}\text { Basketter et al. } \\
(2014)\end{array}$ & $\begin{array}{l}\text { ICCVAM }(2013)+ \\
\operatorname{REACH}(2008-2014)\end{array}$ & Urbisch et al. (2015) \\
\hline
\end{tabular}




\begin{tabular}{|c|c|c|c|c|c|c|c|c|c|}
\hline Compound name & SMILES & $\begin{array}{l}\text { Human } \\
\text { result }\end{array}$ & $\begin{array}{l}\text { LLNA } \\
\text { result }\end{array}$ & DPRA & KeratinoSens & h-CLAT & $\begin{array}{l}\text { Human } \\
\text { reference }\end{array}$ & LLNA reference & Non-animal reference \\
\hline Salicylic acid & $\mathrm{OC}(=0) \mathrm{c} 1 \mathrm{ccccc} 10$ & $\begin{array}{l}\text { Non- } \\
\text { sensitizer }\end{array}$ & $\begin{array}{l}\text { Non- } \\
\text { sensitizer }\end{array}$ & Positive & Negative & Positive & $\begin{array}{l}\text { ICCVAM (2011) } \\
\text { and Basketter et } \\
\text { al. (2014) }\end{array}$ & ICCVAM (2013) & Urbisch et al. (2015) \\
\hline Benzoic acid & $\mathrm{OC}(=0) \operatorname{coccccc} 1$ & $\begin{array}{l}\text { Non- } \\
\text { sensitizer }\end{array}$ & $\begin{array}{l}\text { Non- } \\
\text { sensitizer }\end{array}$ & Positive & Negative & Negative & ICCVAM (2011) & ICCVAM (2013) & Urbisch et al. (2015) \\
\hline Benzaldehyde & $\mathrm{O}=\mathrm{Cc} 1 \operatorname{ccccc} 1$ & $\begin{array}{l}\text { Non- } \\
\text { sensitizer }\end{array}$ & $\begin{array}{l}\text { Non- } \\
\text { sensitizer }\end{array}$ & Negative & Positive & Positive & $\begin{array}{l}\text { Basketter et al. } \\
(2014)\end{array}$ & ICCVAM (2013) & Urbisch et al. (2015) \\
\hline Propylparaben & $\operatorname{ccCOC}(=0) \operatorname{c1ccc}(0) \operatorname{cc} 1$ & $\begin{array}{l}\text { Non- } \\
\text { sensitizer }\end{array}$ & $\begin{array}{l}\text { Non- } \\
\text { sensitizer }\end{array}$ & Negative & Positive & Positive & $\begin{array}{l}\text { Basketter et al. } \\
\text { (2014) }\end{array}$ & $\begin{array}{l}\text { ICCVAM }(2013)+ \\
\text { REACH }(2008-2014)\end{array}$ & Urbisch et al. (2015) \\
\hline $\begin{array}{l}\text { 4- } \\
\text { Methoxyacetophenone }\end{array}$ & $\operatorname{COc} 1 \operatorname{ccc}(\operatorname{cc} 1) C(C)=0$ & $\begin{array}{l}\text { Non- } \\
\text { sensitizer }\end{array}$ & $\begin{array}{l}\text { Non- } \\
\text { sensitizer }\end{array}$ & Negative & Positive & Negative & ICCVAM (2011) & ICCVAM (2013) & Urbisch et al. (2015) \\
\hline 6-Methylcoumarin & $\begin{array}{l}\mathrm{Cc} 1 \mathrm{ccc} 2 \mathrm{OC}(=0) \mathrm{C}=\mathrm{Cc} 2 \mathrm{c} \\
1\end{array}$ & $\begin{array}{l}\text { Non- } \\
\text { sensitizer }\end{array}$ & $\begin{array}{l}\text { Non- } \\
\text { sensitizer }\end{array}$ & Negative & Positive & Negative & ICCVAM (2011) & ICCVAM (2013) & Urbisch et al. (2015) \\
\hline Ethyl vanillin & $\operatorname{ccOc} 1 \mathrm{cc}(\mathrm{C}=0) \operatorname{ccc} 10$ & $\begin{array}{l}\text { Non- } \\
\text { sensitizer }\end{array}$ & $\begin{array}{l}\text { Non- } \\
\text { sensitizer }\end{array}$ & Negative & Positive & Negative & ICCVAM (2011) & $\begin{array}{l}\text { ICCVAM }(2013)+ \\
\text { REACH }(2008-2014)\end{array}$ & Urbisch et al. (2015) \\
\hline Hexane & CCCCCC & $\begin{array}{l}\text { Non- } \\
\text { sensitizer }\end{array}$ & $\begin{array}{l}\text { Non- } \\
\text { sensitizer }\end{array}$ & Negative & Positive & & $\begin{array}{l}\text { ICCVAM (2011) } \\
\text { and Basketter et } \\
\text { al. (2014) }\end{array}$ & $\begin{array}{l}\text { ICCVAM (2013) + } \\
\text { REACH }(2008-2014)\end{array}$ & Urbisch et al. (2015) \\
\hline Chlorobenzene & Clc1ccccc1 & $\begin{array}{l}\text { Non- } \\
\text { sensitizer }\end{array}$ & $\begin{array}{l}\text { Non- } \\
\text { sensitizer }\end{array}$ & Negative & Negative & Positive & $\begin{array}{l}\text { Basketter and } \\
\text { Kimber (2006) }\end{array}$ & ICCVAM (2013) & Urbisch et al. (2015) \\
\hline Diethyl phthalate & $\begin{array}{l}\operatorname{cCOC}(=0) \operatorname{c1ccccc} 1 \mathrm{C}(= \\
\text { 0)OCC }\end{array}$ & $\begin{array}{l}\text { Non- } \\
\text { sensitizer }\end{array}$ & $\begin{array}{l}\text { Non- } \\
\text { sensitizer }\end{array}$ & Negative & Negative & Positive & $\begin{array}{l}\text { ICCVAM (2011) } \\
\text { and Basketter et } \\
\text { al. (2014) }\end{array}$ & $\begin{array}{l}\text { ICCVAM }(2013)+ \\
\text { REACH }(2008-2014)\end{array}$ & Urbisch et al. (2015) \\
\hline Octanoic acid & $\operatorname{cccccccc}(0)=0$ & $\begin{array}{l}\text { Non- } \\
\text { sensitizer }\end{array}$ & $\begin{array}{l}\text { Non- } \\
\text { sensitizer }\end{array}$ & Negative & Negative & Positive & $\begin{array}{l}\text { ICCVAM (2011) } \\
\text { and Basketter et } \\
\text { al. (2014) }\end{array}$ & ICCVAM (2013) & Urbisch et al. (2015) \\
\hline 1-Butanol & CCCCO & $\begin{array}{l}\text { Non- } \\
\text { sensitizer }\end{array}$ & $\begin{array}{l}\text { Non- } \\
\text { sensitizer }\end{array}$ & Negative & Negative & Negative & $\begin{array}{l}\text { ICCVAM (2011) } \\
\text { and Basketter et } \\
\text { al. (2014) }\end{array}$ & ICCVAM (2013) & Urbisch et al. (2015) \\
\hline Glycerol & $\mathrm{OCC}(\mathrm{O}) \mathrm{CO}$ & $\begin{array}{l}\text { Non- } \\
\text { sensitizer }\end{array}$ & $\begin{array}{l}\text { Non- } \\
\text { sensitizer }\end{array}$ & Negative & Negative & Negative & $\begin{array}{l}\text { ICCVAM (2011) } \\
\text { and Basketter et } \\
\text { al. (2014) }\end{array}$ & $\begin{array}{l}\text { ICCVAM }(2013)+ \\
\text { REACH }(2008-2014)\end{array}$ & Urbisch et al. (2015) \\
\hline Isopropanol & $\mathrm{CC}(\mathrm{C}) \mathrm{O}$ & $\begin{array}{l}\text { Non- } \\
\text { sensitizer }\end{array}$ & $\begin{array}{l}\text { Non- } \\
\text { sensitizer }\end{array}$ & Negative & Negative & Negative & $\begin{array}{l}\text { ICCVAM (2011) } \\
\text { and Basketter et } \\
\text { al. (2014) }\end{array}$ & ICCVAM (2013) & Urbisch et al. (2015) \\
\hline Lactic acid & $\mathrm{CC}(\mathrm{O}) \mathrm{C}(\mathrm{O})=\mathrm{O}$ & $\begin{array}{l}\text { Non- } \\
\text { sensitizer }\end{array}$ & $\begin{array}{l}\text { Non- } \\
\text { sensitizer }\end{array}$ & Negative & Negative & Negative & $\begin{array}{l}\text { Basketter et al. } \\
\text { (2014) }\end{array}$ & ICCVAM (2013) & Urbisch et al. (2015) \\
\hline Methyl salicylate & $\operatorname{COC}(=0) \operatorname{cocccc} 10$ & $\begin{array}{l}\text { Non- } \\
\text { sensitizer }\end{array}$ & $\begin{array}{l}\text { Non- } \\
\text { sensitizer }\end{array}$ & Negative & Negative & Negative & $\begin{array}{l}\text { ICCVAM (2011) } \\
\text { and Basketter et } \\
\text { al. (2014) }\end{array}$ & ICCVAM (2013) & Urbisch et al. (2015) \\
\hline Propylene glycol & $\mathrm{CC}(\mathrm{O}) \mathrm{CO}$ & $\begin{array}{l}\text { Non- } \\
\text { sensitizer }\end{array}$ & $\begin{array}{l}\text { Non- } \\
\text { sensitizer }\end{array}$ & Negative & Negative & Negative & $\begin{array}{l}\text { ICCVAM (2011) } \\
\text { and Basketter et } \\
\text { al. (2014) }\end{array}$ & ICCVAM (2013) & Urbisch et al. (2015) \\
\hline
\end{tabular}




\begin{tabular}{|c|c|c|c|c|c|c|c|c|c|}
\hline Compound name & SMILES & $\begin{array}{l}\text { Human } \\
\text { result }\end{array}$ & $\begin{array}{l}\text { LLNA } \\
\text { result }\end{array}$ & DPRA & KeratinoSens & h-CLAT & $\begin{array}{l}\text { Human } \\
\text { reference }\end{array}$ & LLNA reference & Non-animal reference \\
\hline Sulfanilic acid & $\begin{array}{l}\operatorname{Nc1} 1 \operatorname{ccc}(\operatorname{cc} 1) S(O)(=0)= \\
O\end{array}$ & $\begin{array}{l}\text { Non- } \\
\text { sensitizer }\end{array}$ & $\begin{array}{l}\text { Non- } \\
\text { sensitizer }\end{array}$ & Negative & Negative & Negative & ICCVAM (2011) & $\begin{array}{l}\text { ICCVAM }(2013)+ \\
\text { REACH }(2008-2014)\end{array}$ & Urbisch et al. (2015) \\
\hline p-Aminobenzoic acid & $\operatorname{Nc} 1 \mathrm{ccc}(\operatorname{cc} 1) \mathrm{C}(0)=0$ & $\begin{array}{l}\text { Non- } \\
\text { sensitizer }\end{array}$ & $\begin{array}{l}\text { Non- } \\
\text { sensitizer }\end{array}$ & Negative & Negative & & $\begin{array}{l}\text { ICCVAM (2011) } \\
\text { and Basketter et } \\
\text { al. (2014) }\end{array}$ & ICCVAM (2013) & Urbisch et al. (2015) \\
\hline Dimethyl sulfoxide & $\operatorname{CS}(\mathrm{C})=0$ & $\begin{array}{l}\text { Non- } \\
\text { sensitizer }\end{array}$ & $\begin{array}{l}\text { Non- } \\
\text { sensitizer }\end{array}$ & & & & ICCVAM (2011) & $\begin{array}{l}\text { ICCVAM }(2013)+ \\
\text { REACH }(2008-2014)\end{array}$ & \\
\hline Hydrocortisone & $\begin{array}{l}\mathrm{CC} 12 \mathrm{CC}(0) \mathrm{C} 3 \mathrm{C}(\mathrm{CCC} 4= \\
\mathrm{CC}(=0) \mathrm{CCC} 34 \mathrm{C}) \mathrm{C} 1 \mathrm{CCC} \\
2(\mathrm{O}) \mathrm{C}(=0) \mathrm{CO}\end{array}$ & $\begin{array}{l}\text { Non- } \\
\text { sensitizer }\end{array}$ & $\begin{array}{l}\text { Non- } \\
\text { sensitizer }\end{array}$ & & & & ICCVAM (2011) & ICCVAM (2013) & \\
\hline Lead acetate & $C C(O)=0$ & $\begin{array}{l}\text { Non- } \\
\text { sensitizer }\end{array}$ & $\begin{array}{l}\text { Non- } \\
\text { sensitizer }\end{array}$ & & & & ICCVAM (2011) & ICCVAM (2013) & \\
\hline Tween 80 & $\begin{array}{l}\text { CCCCCCCCCC=CCCCCCC } \\
\mathrm{CC}(=0) \text { OCCOCC (OCCO) } \\
\mathrm{C1OC}(\mathrm{CC} 10 \mathrm{OCC}) \text { ) OCCO }\end{array}$ & $\begin{array}{l}\text { Non- } \\
\text { sensitizer }\end{array}$ & & Negative & Positive & Negative & ICCVAM (2011) & & Urbisch et al. (2015) \\
\hline Xylene & $\mathrm{Cc} 1 \mathrm{cccc}(\mathrm{C}) \mathrm{c} 1$ & $\begin{array}{l}\text { Non- } \\
\text { sensitizer }\end{array}$ & & Negative & Negative & & $\begin{array}{l}\text { ICCVAM (2011) } \\
\text { and Basketter et } \\
\text { al. (2014) }\end{array}$ & & Urbisch et al. (2015) \\
\hline trans- $\beta$ Damascone & $\begin{array}{l}\mathrm{CC}=\mathrm{CC}(=0) \mathrm{C} 1=\mathrm{C}(\mathrm{C}) \mathrm{CCC} \\
\mathrm{C} 1(\mathrm{C}) \mathrm{C}\end{array}$ & $\begin{array}{l}\text { Non- } \\
\text { sensitizer }\end{array}$ & & & Positive & Positive & ICCVAM (2011) & & Urbisch et al. (2015) \\
\hline Citronellal & $\mathrm{CC}(\mathrm{CCC}=\mathrm{C}(\mathrm{C}) \mathrm{C}) \mathrm{CC}=\mathrm{O}$ & $\begin{array}{l}\text { Non- } \\
\text { sensitizer }\end{array}$ & & & & & ICCVAM (2011) & & \\
\hline Damascone & $\begin{array}{l}\mathrm{CC}=\mathrm{CC}(=\mathrm{O}) \mathrm{C} 1=\mathrm{C}(\mathrm{C}) \mathrm{C}=\mathrm{C} \\
\mathrm{CC} 1(\mathrm{C}) \mathrm{C}\end{array}$ & $\begin{array}{l}\text { Non- } \\
\text { sensitizer }\end{array}$ & & & & & ICCVAM (2011) & & \\
\hline Isocyclocitral & $\mathrm{CC} 1 \mathrm{C}(\mathrm{CC}=\mathrm{C}(\mathrm{C}) \mathrm{C} 1 \mathrm{C}) \mathrm{C}=\mathrm{O}$ & $\begin{array}{l}\text { Non- } \\
\text { sensitizer }\end{array}$ & & & & & ICCVAM (2011) & & \\
\hline Majantal & $\mathrm{Cc} 1 \mathrm{cccc}(\mathrm{CC}(\mathrm{C})(\mathrm{C}) \mathrm{CO}) \mathrm{c} 1$ & $\begin{array}{l}\text { Non- } \\
\text { sensitizer }\end{array}$ & & & & & ICCVAM (2011) & & \\
\hline $\begin{array}{l}\text { Methoxy } \\
\text { dicyclopentadiene } \\
\text { carboxaldehyde }\end{array}$ & $\begin{array}{l}\mathrm{COC} 1 \mathrm{CC} 2 \mathrm{CC} 1 \mathrm{C} 1 \mathrm{CC}(\mathrm{CC} 2 \\
\text { 1) } \mathrm{C}=0\end{array}$ & $\begin{array}{l}\text { Non- } \\
\text { sensitizer }\end{array}$ & & & & & ICCVAM (2011) & & \\
\hline trans- $\alpha$ Damascone & $\begin{array}{l}\mathrm{CC}=\mathrm{CC}(=0) \mathrm{C} 1 \mathrm{C}(\mathrm{C})=\mathrm{CCC} \\
\mathrm{C} 1(\mathrm{C}) \mathrm{C}\end{array}$ & $\begin{array}{l}\text { Non- } \\
\text { sensitizer }\end{array}$ & & & & & ICCVAM (2011) & & \\
\hline 1-Naphthol & Oc1cccc2ccccc12 & & Sensitizer & Positive & Positive & Positive & & ICCVAM (2013) & Urbisch et al. (2015) \\
\hline $\begin{array}{l}\text { 1-Phenyl-1,2- } \\
\text { propanedione }\end{array}$ & $\mathrm{OC}(=\mathrm{C}) \mathrm{C}(=0) \mathrm{c} 1 \mathrm{ccccc} 1$ & & Sensitizer & Positive & Positive & Positive & & ICCVAM (2013) & Urbisch et al. (2015) \\
\hline 2,3-Butanedione & $C C(=O) C(O)=C$ & & Sensitizer & Positive & Positive & Positive & & ICCVAM (2013) & Urbisch et al. (2015) \\
\hline 2,4-Heptadienal & $\mathrm{CCC}=\mathrm{CC}=\mathrm{CC}=\mathrm{O}$ & & Sensitizer & Positive & Positive & Positive & & ICCVAM (2013) & Urbisch et al. (2015) \\
\hline 2-Ethylhexylacrylate & $\operatorname{ccccc}(\operatorname{cc}) \operatorname{coc}(=0) C=C$ & & Sensitizer & Positive & Positive & Positive & & $\begin{array}{l}\text { ICCVAM }(2013)+ \\
\text { REACH }(2008-2014)\end{array}$ & Urbisch et al. (2015) \\
\hline
\end{tabular}




\begin{tabular}{|c|c|c|c|c|c|c|c|c|c|}
\hline Compound name & SMILES & $\begin{array}{l}\text { Human } \\
\text { result }\end{array}$ & $\begin{array}{l}\text { LLNA } \\
\text { result }\end{array}$ & DPRA & KeratinoSens & h-CLAT & $\begin{array}{l}\text { Human } \\
\text { reference }\end{array}$ & LLNA reference & Non-animal reference \\
\hline $\begin{array}{l}\text { 4-(N-Ethyl-N-2-methan- } \\
\text { sulfamido-ethyl)-2- } \\
\text { methyl-1,4,- } \\
\text { phenylenediamine } \\
\text { (CD3) }\end{array}$ & $\begin{array}{l}\operatorname{CCN}(\operatorname{CCNS}(\mathrm{C})(=\mathrm{O})=0) \mathrm{c} \\
1 \operatorname{ccc}(\mathrm{N}) \mathrm{c}(\mathrm{C}) \mathrm{c1}\end{array}$ & & Sensitizer & Positive & Positive & Positive & & ICCVAM (2013) & Urbisch et al. (2015) \\
\hline 4-Nitrobenzyl bromide & $\operatorname{BrCc} 1 \operatorname{ccc}(\operatorname{cc} 1) \mathrm{N}(=0)=0$ & & Sensitizer & Positive & Positive & Positive & & ICCVAM (2013) & Urbisch et al. (2015) \\
\hline Butyl acrylate & $\operatorname{ccccoc}(=0) C=C$ & & Sensitizer & Positive & Positive & Positive & & $\begin{array}{l}\text { ICCVAM }(2013)+ \\
\text { REACH }(2008-2014)\end{array}$ & Urbisch et al. (2015) \\
\hline Chlorothalonil & $\begin{array}{l}\mathrm{Clc1c}(\mathrm{Cl}) \mathrm{c}(\mathrm{C \# N}) \mathrm{c}(\mathrm{Cl}) \mathrm{c}(\mathrm{C} \\
\# \mathrm{~N}) \mathrm{c} 1 \mathrm{Cl}\end{array}$ & & Sensitizer & Positive & Positive & Positive & & ICCVAM (2013) & Urbisch et al. (2015) \\
\hline Diethyl sulfate & $\operatorname{ccos}(=0)(=0) O C C$ & & Sensitizer & Positive & Positive & Positive & & ICCVAM (2013) & Urbisch et al. (2015) \\
\hline Diethylacetaldehyde & $\mathrm{CCC}(\mathrm{CC}) \mathrm{C}=\mathrm{O}$ & & Sensitizer & Positive & Positive & Positive & & ICCVAM (2013) & Urbisch et al. (2015) \\
\hline $\begin{array}{l}\text { lodopropynyl } \\
\text { butylcarbamate }\end{array}$ & $\mathrm{CCCCNC}(=0) \mathrm{OC \# CCl}$ & & Sensitizer & Positive & Positive & Positive & & ICCVAM (2013) & Urbisch et al. (2015) \\
\hline Tropolone & $\mathrm{OC} 1=\mathrm{CC}=\mathrm{CC}=\mathrm{CC} 1=\mathrm{O}$ & & Sensitizer & Positive & Positive & Positive & & ICCVAM (2013) & Urbisch et al. (2015) \\
\hline $\begin{array}{l}\alpha- \\
\text { Methylphenylacetaldeh } \\
\text { yde }\end{array}$ & $\mathrm{CC}(\mathrm{C}=0) \mathrm{c} 1 \mathrm{ccccc} 1$ & & Sensitizer & Positive & Positive & Positive & & ICCVAM (2013) & Urbisch et al. (2015) \\
\hline 1-Bromohexane & CCCCCCBr & & Sensitizer & Positive & Positive & Negative & & ICCVAM (2013) & Urbisch et al. (2015) \\
\hline $\begin{array}{l}\text { 2,2-bis-[4-(2-hydroxy-3 } \\
\text { methacryloxypropoxy)p } \\
\text { henyl)]-propane }\end{array}$ & $\begin{array}{l}\mathrm{CC}(=\mathrm{C}) \mathrm{C}(=\mathrm{O}) \mathrm{OCC}(\mathrm{O}) \mathrm{CO} \\
\mathrm{c} 1 \mathrm{ccc}(\mathrm{cc} 1) \mathrm{C}(\mathrm{C})(\mathrm{C}) \mathrm{c} 1 \mathrm{ccc} \\
(\mathrm{OCC}(\mathrm{O}) \operatorname{cOC}(=0) \mathrm{C}(\mathrm{C})= \\
\mathrm{C}) \mathrm{cc} 1\end{array}$ & & Sensitizer & Positive & Positive & Negative & & ICCVAM (2013) & Urbisch et al. (2015) \\
\hline $\begin{array}{l}\text { Methyl } \\
\text { methanesulfonate }\end{array}$ & $\cos (C)(=0)=0$ & & Sensitizer & Positive & Positive & Negative & & ICCVAM (2013) & Urbisch et al. (2015) \\
\hline $\begin{array}{l}\text { 1,1,3-Trimethyl-2- } \\
\text { formylcyclohexa-2,4- } \\
\text { diene (Safranal) }\end{array}$ & $\begin{array}{l}\mathrm{CC} 1=\mathrm{C}(\mathrm{C}=\mathrm{O}) \mathrm{C}(\mathrm{C})(\mathrm{C}) \mathrm{CC}= \\
\mathrm{C} 1\end{array}$ & & Sensitizer & Positive & Positive & & & ICCVAM (2013) & Urbisch et al. (2015) \\
\hline $\begin{array}{l}\text { 2,4-Dinitrobenzene } \\
\text { sulfonic acid }\end{array}$ & $\begin{array}{l}\mathrm{OS}(=0)(=0) \operatorname{c1ccc}(\operatorname{cc} 1 \mathrm{~N} \\
(=0)=0) \mathrm{N}(=0)=\mathrm{O}\end{array}$ & & Sensitizer & Positive & Positive & & & ICCVAM (2013) & Urbisch et al. (2015) \\
\hline 2-Methylundecanal & $\operatorname{cccccccccc}(C) \mathrm{C}=0$ & & Sensitizer & Positive & Positive & & & $\begin{array}{l}\text { ICCVAM }(2013)+ \\
\text { REACH }(2008-2014)\end{array}$ & Urbisch et al. (2015) \\
\hline 3-Methylcatechol & $\operatorname{Cc} 1 \mathrm{cccc}(0) \mathrm{c} 10$ & & Sensitizer & Positive & Positive & & & $\begin{array}{l}\text { J Appl Toxicol, v } \\
\text { 33(11), 1353-64, } 2013 .\end{array}$ & Urbisch et al. (2015) \\
\hline $\begin{array}{l}\text { 4-(Methylamino)phenol } \\
\text { sulfate (metol) }\end{array}$ & $\mathrm{CNc1ccc}(\mathrm{O}) \mathrm{cc} 1$ & & Sensitizer & Positive & Positive & & & ICCVAM (2013) & Urbisch et al. (2015) \\
\hline $\begin{array}{l}\text { 4-Amimo-3- } \\
\text { methylphenol }\end{array}$ & $\operatorname{Cc} 1 \mathrm{cc}(\mathrm{O}) \operatorname{ccc} 1 \mathrm{~N}$ & & Sensitizer & Positive & Positive & & & ICCVAM (2013) & Urbisch et al. (2015) \\
\hline 4-Vinyl pyridine & $\mathrm{C}=\mathrm{Cc} 1 \mathrm{ccncc} 1$ & & Sensitizer & Positive & Positive & & & REACH (2008-2014) & Urbisch et al. (2015) \\
\hline $\begin{array}{l}\text { 5-Amino-2- } \\
\text { methylphenol }\end{array}$ & $\operatorname{Cc} 1 \mathrm{ccc}(\mathrm{N}) \operatorname{cc} 10$ & & Sensitizer & Positive & Positive & & & ICCVAM (2013) & Urbisch et al. (2015) \\
\hline
\end{tabular}




\begin{tabular}{|c|c|c|c|c|c|c|c|c|c|}
\hline Compound name & SMILES & $\begin{array}{l}\text { Human } \\
\text { result }\end{array}$ & $\begin{array}{l}\text { LLNA } \\
\text { result }\end{array}$ & DPRA & KeratinoSens & h-CLAT & $\begin{array}{l}\text { Human } \\
\text { reference }\end{array}$ & LLNA reference & Non-animal reference \\
\hline Farnesal & $\begin{array}{l}\mathrm{CC}(\mathrm{C})=\mathrm{CCCC}(\mathrm{C})=\mathrm{CCCC}( \\
\mathrm{C})=\mathrm{CC}=\mathrm{O}\end{array}$ & & Sensitizer & Positive & Positive & & & ICCVAM (2013) & Urbisch et al. (2015) \\
\hline $\begin{array}{l}\text { Fluorescein-5- } \\
\text { isothiocyanate }\end{array}$ & $\begin{array}{l}\text { Oc1ccc2c(Oc3cc }(0) c c c \\
3 \mathrm{c} 22 \mathrm{OC}(=\mathrm{O}) \mathrm{c} 3 \mathrm{cc}(\mathrm{ccc} 23 \\
) \mathrm{N}=\mathrm{C}=\mathrm{S}) \mathrm{c} 1\end{array}$ & & Sensitizer & Positive & Positive & & & ICCVAM (2013) & Urbisch et al. (2015) \\
\hline Methyl acrylate & $\operatorname{COC}(=0) C=C$ & & Sensitizer & Positive & Positive & & & $\begin{array}{l}\text { ICCVAM }(2013)+ \\
\text { REACH }(2008-2014)\end{array}$ & Urbisch et al. (2015) \\
\hline $\begin{array}{l}\mathrm{N}, \mathrm{N} \text {-dimethyl-4- } \\
\text { nitrosoaniline }\end{array}$ & $\mathrm{CN}(\mathrm{C}) \operatorname{c1ccc}(\mathrm{cc} 1) \mathrm{N}=\mathrm{O}$ & & Sensitizer & Positive & Positive & & & $\begin{array}{l}\text { J Appl Toxicol, v } \\
33(11), 1353-64,2013 .\end{array}$ & Urbisch et al. (2015) \\
\hline trans-2-Decenal & $\operatorname{CCCCCCCC}=\mathrm{CC}=\mathrm{O}$ & & Sensitizer & Positive & Positive & & & ICCVAM (2013) & Urbisch et al. (2015) \\
\hline $\begin{array}{l}\text { 1,2-cyclohexane } \\
\text { dicarboxylic anhydride }\end{array}$ & $\begin{array}{l}\mathrm{O}=\mathrm{C} 1 \mathrm{OC}(=\mathrm{O}) \mathrm{C} 2 \mathrm{CCCCC} 1 \\
2\end{array}$ & & Sensitizer & Positive & Negative & & & $\begin{array}{l}\text { J Appl Toxicol, v } \\
\text { 33(11), 1353-64, } 2013 .\end{array}$ & Urbisch et al. (2015) \\
\hline Squaric acid & $O C 1=C(0) C(=0) C 1=0$ & & Sensitizer & Positive & Negative & & & ICCVAM (2013) & Urbisch et al. (2015) \\
\hline 4-Allylanisole & $\operatorname{cOc} 1 \mathrm{ccc}(\mathrm{CC}=\mathrm{C}) \mathrm{cc} 1$ & & Sensitizer & Positive & & Positive & & ICCVAM (2013) & Urbisch et al. (2015) \\
\hline Clotrimazole & $\begin{array}{l}\text { Clc1ccccc1C(c1cccco1)( } \\
\mathrm{c} 1 \mathrm{ccccc} 1) n 1 \mathrm{ccnc} 1\end{array}$ & & Sensitizer & Positive & & Negative & & ICCVAM (2013) & Urbisch et al. (2015) \\
\hline 1,6-Diisocyanatohexane & $\mathrm{O}=\mathrm{C}=\mathrm{NCCCCCCN}=\mathrm{C}=\mathrm{O}$ & & Sensitizer & Positive & & & & REACH (2008-2014) & Urbisch et al. (2015) \\
\hline 1-lodohexadecane & CCCCCCCCCCCCCCCCI & & Sensitizer & Positive & & & & ICCVAM (2013) & Urbisch et al. (2015) \\
\hline $\begin{array}{l}\text { 2-propylheptyl prop-2- } \\
\text { enoate }\end{array}$ & $\begin{array}{l}\mathrm{CCCCCC}(\mathrm{CCC}) \operatorname{COC}(=0) \\
\mathrm{C}=\mathrm{C}\end{array}$ & & Sensitizer & Positive & & & & REACH (2008-2014) & Urbisch et al. (2015) \\
\hline Oxalic Acid & $O C(=0) C(O)=0$ & & Sensitizer & Negative & Positive & Positive & & ICCVAM (2013) & Urbisch et al. (2015) \\
\hline Undec-10-enal & $\mathrm{C}=\mathrm{CCCCCCCCCC}=\mathrm{O}$ & & Sensitizer & Negative & Positive & Negative & & ICCVAM (2013) & Urbisch et al. (2015) \\
\hline $\begin{array}{l}\text { 7,12- } \\
\text { Dimethylbenz[a]anthra } \\
\text { cene }\end{array}$ & $\begin{array}{l}\mathrm{Cc} 1 \mathrm{c} 2 \mathrm{ccccc} 2 \mathrm{c}(\mathrm{C}) \mathrm{c} 2 \mathrm{c} 1 \mathrm{cc} \\
\mathrm{c} 1 \mathrm{cccc} 21\end{array}$ & & Sensitizer & Negative & Positive & & & ICCVAM (2013) & Urbisch et al. (2015) \\
\hline Dihydroeugenol & $\operatorname{ccCc} 1 \mathrm{ccc}(\mathrm{O}) \mathrm{c}(\mathrm{OC}) \mathrm{c1}$ & & Sensitizer & Negative & Positive & & & ICCVAM (2013) & Urbisch et al. (2015) \\
\hline Undecylenic acid & $\mathrm{OC}(=\mathrm{O}) \mathrm{CCCCCCCCC}=\mathrm{C}$ & & Sensitizer & Negative & Positive & & & ICCVAM (2013) & Urbisch et al. (2015) \\
\hline cis-6-Nonenal & $\mathrm{CCC}=\mathrm{CCCCCC}=\mathrm{O}$ & & Sensitizer & Negative & Positive & & & ICCVAM (2013) & Urbisch et al. (2015) \\
\hline Methyl pyruvate & $\operatorname{coc}(=0) C(0)=C$ & & Sensitizer & Negative & Negative & Negative & & ICCVAM (2013) & Urbisch et al. (2015) \\
\hline $\mathrm{N}, \mathrm{N}$-dibutylaniline & $\mathrm{CCCCN}(\mathrm{CCCC}) \mathrm{c} 1 \mathrm{ccccc} 1$ & & Sensitizer & Negative & Negative & Negative & & ICCVAM (2013) & Urbisch et al. (2015) \\
\hline $\begin{array}{l}\text { 2,2,6,6-Tetramethyl- } \\
\text { heptane-3,5-dione }\end{array}$ & $\begin{array}{l}\mathrm{CC}(\mathrm{C})(\mathrm{C}) \mathrm{C}(\mathrm{O})=\mathrm{CC}(=\mathrm{O}) \mathrm{C}( \\
\mathrm{C})(\mathrm{C}) \mathrm{C}\end{array}$ & & Sensitizer & Negative & Negative & & & ICCVAM (2013) & Urbisch et al. (2015) \\
\hline $\begin{array}{l}\text { Squaric acid diethyl } \\
\text { ester }\end{array}$ & $\begin{array}{l}\operatorname{CCOC} 1=C(O C C) C(=0) C \\
1=0\end{array}$ & & Sensitizer & Negative & Negative & & & $\begin{array}{l}\text { J Appl Toxicol, v } \\
\text { 33(11), 1353-64, } 2013 .\end{array}$ & Urbisch et al. (2015) \\
\hline Phenol, 2,2'-azobis- & $\begin{array}{l}\text { Oc1 } 1 \operatorname{ccccc} 1 \mathrm{~N}=\mathrm{Nc} 1 \mathrm{ccccc} \\
10\end{array}$ & & Sensitizer & Negative & & Positive & & ICCVAM (2013) & Urbisch et al. (2015) \\
\hline
\end{tabular}




\begin{tabular}{|c|c|c|c|c|c|c|c|c|c|}
\hline Compound name & SMILES & $\begin{array}{l}\text { Human } \\
\text { result }\end{array}$ & $\begin{array}{l}\text { LLNA } \\
\text { result }\end{array}$ & DPRA & KeratinoSens & h-CLAT & $\begin{array}{l}\text { Human } \\
\text { reference }\end{array}$ & LLNA reference & Non-animal reference \\
\hline$\alpha$-Tocopherol & $\begin{array}{l}\mathrm{CC}(\mathrm{C}) \mathrm{CCCC}(\mathrm{C}) \mathrm{CcCC}(\mathrm{C}) \mathrm{C} \\
\mathrm{CCC} 1(\mathrm{C}) \mathrm{CCc} 2 \mathrm{c}(\mathrm{C}) \mathrm{c}(\mathrm{O}) \mathrm{cl} \\
\mathrm{C}) \mathrm{c}(\mathrm{C}) \mathrm{c} 2 \mathrm{O} 1\end{array}$ & & Sensitizer & Negative & & Negative & & ICCVAM (2013) & Urbisch et al. (2015) \\
\hline $\begin{array}{l}2,4- \\
\text { Dinitrofluorobenzene }\end{array}$ & $\begin{array}{l}\text { Fc1 } 1 \operatorname{ccc}(\operatorname{cc} 1 N(=0)=0) N( \\
=0)=0\end{array}$ & & Sensitizer & & Positive & Positive & & ICCVAM (2013) & Urbisch et al. (2015) \\
\hline $\begin{array}{l}\text { 5-Methyl-2-phenyl-2- } \\
\text { hexenal }\end{array}$ & $\begin{array}{l}\mathrm{CC}(\mathrm{C}) \mathrm{CC}=\mathrm{C}(\mathrm{C}=\mathrm{O}) \mathrm{c} 1 \mathrm{cccc} \\
\mathrm{c1}\end{array}$ & & Sensitizer & & Positive & Positive & & ICCVAM (2013) & Urbisch et al. (2015) \\
\hline Benzo[a]pyrene & $\begin{array}{l}\mathrm{c} 1 \mathrm{ccc} 2 \mathrm{c}(\mathrm{c} 1) \mathrm{cc} 1 \mathrm{ccc} 3 \mathrm{ccc} \\
\mathrm{c} 4 \mathrm{ccc} 2 \mathrm{c} 1 \mathrm{c} 34\end{array}$ & & Sensitizer & & Positive & Positive & & ICCVAM (2013) & Urbisch et al. (2015) \\
\hline $\begin{array}{l}\text { 3-Methyl-1- } \\
\text { phenylpyrazolone }\end{array}$ & $\begin{array}{l}\mathrm{CC} 1=\mathrm{CC}(=0) \mathrm{N}(\mathrm{N} 1) \mathrm{c} 1 \mathrm{cc} \\
\mathrm{ccc} 1\end{array}$ & & Sensitizer & & Negative & Negative & & ICCVAM (2013) & Urbisch et al. (2015) \\
\hline $\begin{array}{l}\text { 2-Methyl-4H,3,1- } \\
\text { benzoxazin-4-one }\end{array}$ & $\begin{array}{l}\mathrm{CC} 1=\mathrm{Nc} 2 \operatorname{ccccc} 2 \mathrm{C}(=0) \mathrm{O} \\
1\end{array}$ & & Sensitizer & & & Positive & & ICCVAM (2013) & Urbisch et al. (2015) \\
\hline 1-Chloromethylpyrene & $\begin{array}{l}\mathrm{ClCc1} 1 \mathrm{ccc} 2 \mathrm{ccc} 3 \mathrm{cccc} 4 \mathrm{ccc} \\
1 \mathrm{c} 2 \mathrm{c} 34\end{array}$ & & Sensitizer & & & Negative & & ICCVAM (2013) & Urbisch et al. (2015) \\
\hline 12-Bromo-1-dodecanol & OCCCCCCCCCCCCBr & & Sensitizer & & & Negative & & ICCVAM (2013) & Urbisch et al. (2015) \\
\hline $\begin{array}{l}\text { (1S,2S)-2-methylamino- } \\
\text { 1-phenylpropan-1-ol }\end{array}$ & $\mathrm{CNC}(\mathrm{C}) \mathrm{C}(\mathrm{O}) \mathrm{c} 1 \mathrm{ccccc} 1$ & & Sensitizer & & & & & REACH (2008-2014) & \\
\hline $\begin{array}{l}(2,3,4,5,6- \\
\text { pentabromophenyl)met } \\
\text { hyl prop-2-enoate }\end{array}$ & $\begin{array}{l}\mathrm{Brc} 1 \mathrm{c}(\mathrm{Br}) \mathrm{c}(\mathrm{Br}) \mathrm{c}(\mathrm{COC}(= \\
\mathrm{O}) \mathrm{C}=\mathrm{C}) \mathrm{c}(\mathrm{Br}) \mathrm{c} 1 \mathrm{Br}\end{array}$ & & Sensitizer & & & & & REACH (2008-2014) & \\
\hline $\begin{array}{l}\text { (2,3-dimethyl-3- } \\
\text { phenylbutan-2- } \\
\text { yl)benzene }\end{array}$ & $\begin{array}{l}\mathrm{CC}(\mathrm{C})(\mathrm{c} 1 \mathrm{ccccc} 1) \mathrm{C}(\mathrm{C})(\mathrm{C}) \\
\mathrm{c} 1 \mathrm{ccccc} 1\end{array}$ & & Sensitizer & & & & & REACH (2008-2014) & \\
\hline $\begin{array}{l}\text { (2,4-dichlorobenzoyl) } \\
2,4- \\
\text { dichlorobenzenecarbop } \\
\text { eroxoate }\end{array}$ & $\begin{array}{l}\mathrm{Clc1} 1 \mathrm{ccc}(\mathrm{C}(=\mathrm{O}) \mathrm{OOC}(=\mathrm{O}) \\
\operatorname{c2ccc}(\mathrm{Cl}) \operatorname{cc} 2 \mathrm{Cl}) \mathrm{c}(\mathrm{Cl}) \mathrm{c1}\end{array}$ & & Sensitizer & & & & & REACH (2008-2014) & \\
\hline $\begin{array}{l}\text { (2-methyl-4- } \\
\text { phenylpent-4-en-2- } \\
\text { yl)benzene }\end{array}$ & $\begin{array}{l}\mathrm{CC}(\mathrm{C})(\mathrm{CC}(=\mathrm{C}) \operatorname{c1} 1 \mathrm{ccccc} 1) \\
\mathrm{c} 1 \mathrm{ccccc} 1\end{array}$ & & Sensitizer & & & & & REACH (2008-2014) & \\
\hline $\begin{array}{l}\text { (2- } \\
\text { methylpropylidene)[(3- } \\
\{[(2- \\
\text { methylpropylidene)ami } \\
\text { no]methyl\}cyclohexyl)m } \\
\text { ethyl]amine }\end{array}$ & $\begin{array}{l}\mathrm{CC}(\mathrm{C}) \mathrm{C}=\mathrm{NCC} 1 \mathrm{CCCC}(\mathrm{CN} \\
=\mathrm{CC}(\mathrm{C}) \mathrm{C}) \mathrm{C} 1\end{array}$ & & Sensitizer & & & & & REACH (2008-2014) & \\
\hline $\begin{array}{l}\text { (2E)-2-[(2-formyl-4- } \\
\text { hydroxyphenyl)- } \\
\text { methylidene]- } \\
\text { butanedioic acid }\end{array}$ & $\begin{array}{l}\mathrm{OC}(=0) \mathrm{CC}(=\mathrm{Cc} 1 \mathrm{ccc}(0) \mathrm{c} \\
\mathrm{c} 1 \mathrm{C}=0) \mathrm{C}(\mathrm{O})=0\end{array}$ & & Sensitizer & & & & & ICCVAM (2013) & \\
\hline $\begin{array}{l}\text { (2S)-2-[2-[(2S)-oxolan- } \\
2-y \mid] p r o p a n-2- \\
\text { yl]oxolane }\end{array}$ & $\begin{array}{l}\mathrm{CC}(\mathrm{C})(\mathrm{C} 1 \mathrm{CCCO} 1) \mathrm{C} 1 \mathrm{CCC} \\
\mathrm{O} 1\end{array}$ & & Sensitizer & & & & & REACH (2008-2014) & \\
\hline
\end{tabular}




\begin{tabular}{|c|c|c|c|c|c|c|c|c|c|}
\hline Compound name & SMILES & $\begin{array}{l}\text { Human } \\
\text { result }\end{array}$ & $\begin{array}{l}\text { LLNA } \\
\text { result }\end{array}$ & DPRA & KeratinoSens & h-CLAT & $\begin{array}{l}\text { Human } \\
\text { reference }\end{array}$ & LLNA reference & Non-animal reference \\
\hline $\begin{array}{l}\text { (2S,5R)-5-methyl-2- } \\
\text { propan-2-ylcyclohexan- } \\
\text { 1-one }\end{array}$ & $\mathrm{CC}(\mathrm{C}) \mathrm{C} 1 \mathrm{CCC}(\mathrm{C}) \mathrm{CC} 1=0$ & & Sensitizer & & & & & REACH (2008-2014) & \\
\hline $\begin{array}{l}\text { (4-tert-butylcyclohexyl) } \\
\text { (4-tert- } \\
\text { butylcyclohexyl)oxycarb } \\
\text { onyloxy carbonate } \\
\end{array}$ & $\begin{array}{l}\mathrm{CC}(\mathrm{C})(\mathrm{C}) \mathrm{C} 1 \mathrm{CCC}(\mathrm{CC} 1) \mathrm{OC} \\
(=0) \mathrm{OOC}(=0) \mathrm{OC} 1 \mathrm{CCC}( \\
\mathrm{CC} 1) \mathrm{C}(\mathrm{C})(\mathrm{C}) \mathrm{C}\end{array}$ & & Sensitizer & & & & & REACH (2008-2014) & \\
\hline $\begin{array}{l}\text { (4-tert-butylcyclohexyl) } \\
\text { acetate }\end{array}$ & $\begin{array}{l}\mathrm{CC}(=0) 0 \mathrm{OC} 1 \mathrm{CCC}(\mathrm{CC} 1) \mathrm{C}( \\
\mathrm{C})(\mathrm{C}) \mathrm{C}\end{array}$ & & Sensitizer & & & & & REACH (2008-2014) & \\
\hline $\begin{array}{l}\text { (4R,5S)-1,5-Dimethyl-3- } \\
\text { (1-oxo-2-propenyl)-4- } \\
\text { phenyl-2-imidazo- } \\
\text { lidinone }\end{array}$ & $\begin{array}{l}\operatorname{CC1C}(\mathrm{N}(\mathrm{C}(=\mathrm{O}) \mathrm{C}=\mathrm{C}) \mathrm{C}(= \\
\mathrm{O}) \mathrm{N} 1 \mathrm{C}) \operatorname{c1ccccc} 1\end{array}$ & & Sensitizer & & & & & ICCVAM (2013) & \\
\hline $\begin{array}{l}\text { (5R)-5-Isopropenyl-2- } \\
\text { methyl-1-methylene-2- } \\
\text { cyclohexene }\end{array}$ & $\begin{array}{l}\mathrm{CC}(=\mathrm{C}) \mathrm{C} 1 \mathrm{CC}=\mathrm{C}(\mathrm{C}) \mathrm{C}(=\mathrm{C}) \\
\mathrm{C} 1\end{array}$ & & Sensitizer & & & & & ICCVAM (2013) & \\
\hline $\begin{array}{l}\text { (ethoxy- } \\
\text { phenylphosphoryl)- } \\
(2,4,6- \\
\text { trimethylphenyl)metha } \\
\text { none }\end{array}$ & $\begin{array}{l}\operatorname{CCOP}(=0)(C(O)=C 1 C(C) \\
=C C(C)=C C 1=C) \operatorname{c} 1 \operatorname{ccccc} \\
1\end{array}$ & & Sensitizer & & & & & REACH (2008-2014) & \\
\hline (pyridin-3-yl)methanol & OCc1cccnc1 & & Sensitizer & & & & & REACH (2008-2014) & \\
\hline $\begin{array}{l}1,1,2,2- \\
\text { Tetrachloroethene }\end{array}$ & $\mathrm{ClC}(\mathrm{Cl})=\mathrm{C}(\mathrm{Cl}) \mathrm{Cl}$ & & Sensitizer & & & & & REACH (2008-2014) & \\
\hline $\begin{array}{l}\text { 1,1,2,3,3-pentamethyl- } \\
2,5,6,7- \\
\text { tetrahydroinden-4-one }\end{array}$ & $\begin{array}{l}\mathrm{CC} 1 \mathrm{C}(\mathrm{C})(\mathrm{C}) \mathrm{C} 2=\mathrm{C}(\mathrm{C}(=0) \\
\mathrm{CCC} 2) \mathrm{C}(\mathrm{C}) \mathrm{C}\end{array}$ & & Sensitizer & & & & & REACH (2008-2014) & \\
\hline 1,1,2-trichloroethene & $\mathrm{ClC}=\mathrm{C}(\mathrm{Cl}) \mathrm{Cl}$ & & Sensitizer & & & & & REACH (2008-2014) & \\
\hline $\begin{array}{l}\text { 1,1,3,3- } \\
\text { tetramethylbutyl } \\
\text { hydroperoxide }\end{array}$ & $\mathrm{CC}(\mathrm{C})(\mathrm{C}) \mathrm{CC}(\mathrm{C})(\mathrm{C}) \mathrm{OO}$ & & Sensitizer & & & & & REACH (2008-2014) & \\
\hline $\begin{array}{l}\text { 1,1-dimethylethyl 3- } \\
\text { [[[[(3s)-2,3,4,5- } \\
\text { tetrahydro-1-[2-[(1- } \\
\text { methylethyl)phenylami } \\
\text { no]-2-oxoethyl]-2,4- } \\
\text { dioxo-5-phenyl-1h-1,5- } \\
\text { benzodiazepin-3- } \\
\text { yl]amino]carbonyl]amin } \\
\text { o]benzoate }\end{array}$ & $\begin{array}{l}\mathrm{CC}(\mathrm{C}) \mathrm{N}(\mathrm{C}(=\mathrm{O}) \mathrm{CN} 1 \mathrm{c} 2 \mathrm{ccc} \\
\operatorname{cc} 2 \mathrm{~N}(\mathrm{c} 2 \operatorname{ccccc} 2) \mathrm{C}(=\mathrm{O}) \mathrm{C}( \\
\mathrm{NC}(=\mathrm{O}) \mathrm{Nc} 2 \mathrm{cccc}(\mathrm{c} 2) \mathrm{C}= \\
\mathrm{O}=\mathrm{OC}(\mathrm{C})(\mathrm{C}) \mathrm{C}) \mathrm{C} 1=\mathrm{O}) \mathrm{c} 1 \mathrm{c} \\
\operatorname{cccc1}\end{array}$ & & Sensitizer & & & & & ICCVAM (2013) & \\
\hline $\begin{array}{l}\text { 1,2- } \\
\text { Diaminocyclohexane }\end{array}$ & NC1CCCCC1N & & Sensitizer & & & & & ICCVAM (2013) & \\
\hline 1,2-Dichlorobenzene & Clc1ccccc1Cl & & Sensitizer & & & & & REACH (2008-2014) & \\
\hline
\end{tabular}




\begin{tabular}{|c|c|c|c|c|c|c|c|c|c|}
\hline Compound name & SMILES & $\begin{array}{l}\text { Human } \\
\text { result }\end{array}$ & $\begin{array}{l}\text { LLNA } \\
\text { result }\end{array}$ & DPRA & KeratinoSens & h-CLAT & $\begin{array}{l}\text { Human } \\
\text { reference }\end{array}$ & LLNA reference & Non-animal reference \\
\hline 1,2-Dimethylbenzene & Cc1ccccc1C & & Sensitizer & & & & & REACH (2008-2014) & \\
\hline 1,2-Dimethylimidazole & Cc1ncon1C & & Sensitizer & & & & & REACH (2008-2014) & \\
\hline $\begin{array}{l}\text { 1,3,5-trimethyl-1,3,5- } \\
\text { triazinane }\end{array}$ & $\mathrm{CN} 1 \mathrm{CN}(\mathrm{C}) \mathrm{CN}(\mathrm{C}) \mathrm{C} 1$ & & Sensitizer & & & & & REACH (2008-2014) & \\
\hline $\begin{array}{l}\text { 1,3-Benzodioxazole-5- } \\
\text { sulphonyl chloride }\end{array}$ & $\begin{array}{l}\mathrm{CIS}(=0)(=0) \operatorname{c1} 1 \mathrm{ccc} 2 \mathrm{OCO} \\
\mathrm{c} 2 \mathrm{c} 1\end{array}$ & & Sensitizer & & & & & ICCVAM (2013) & \\
\hline 1,3-Dioxol-2-one & $\mathrm{O}=\mathrm{C} 1 \mathrm{OC}=\mathrm{CO} 1$ & & Sensitizer & & & & & REACH (2008-2014) & \\
\hline $\begin{array}{l}\text { 1,3-bis }(3,5,5- \\
\text { trimethylhexanoyloxy)p } \\
\text { ropan-2-yl 3,5,5- } \\
\text { trimethylhexanoate }\end{array}$ & $\begin{array}{l}\mathrm{CC}(\mathrm{CC}(=)) O C C(\mathrm{COC}= \\
\mathrm{O}) \mathrm{CC}(\mathrm{C}) \mathrm{CC}(\mathrm{C})(\mathrm{C}) \mathrm{C}) \mathrm{OC}(= \\
\mathrm{O}) \mathrm{CC}(\mathrm{C}) \mathrm{CC}(\mathrm{C})(\mathrm{C}) \mathrm{C}) \mathrm{CC}(\mathrm{C} \\
)(\mathrm{C}) \mathrm{C}\end{array}$ & & Sensitizer & & & & & REACH (2008-2014) & \\
\hline $\begin{array}{l}\text { 1,3-bis-(2,4- } \\
\text { diaminophenoxy)- } \\
\text { propane }\end{array}$ & $\begin{array}{l}\operatorname{Nc1} 1 \mathrm{ccc}(\mathrm{OCCCO} 2 \mathrm{ccc}(\mathrm{N} \\
\operatorname{ccc} 2 \mathrm{~N}) \mathrm{c}(\mathrm{N}) \mathrm{c1}\end{array}$ & & Sensitizer & & & & & ICCVAM (2013) & \\
\hline $\begin{array}{l}\text { 1,3-di(phenyl)propane- } \\
\text { 1,3-dione }\end{array}$ & $\begin{array}{l}\mathrm{OC}(=\mathrm{CC}(=0) \operatorname{c} 1 \operatorname{ccccc} 1) \mathrm{c} \\
1 \mathrm{ccccc} 1\end{array}$ & & Sensitizer & & & & & REACH (2008-2014) & \\
\hline 1,4-Dimethylbenzene & Cc1ccc(C)cc1 & & Sensitizer & & & & & REACH (2008-2014) & \\
\hline $\begin{array}{l}\text { 1,5,2,4-Dioxadithiane } \\
\text { 2,2,4,4-tetraoxide }\end{array}$ & $\begin{array}{l}\mathrm{O}=\mathrm{S} 1(=0) \mathrm{CS}(=0)(=0) 0 \\
\mathrm{CO} 1\end{array}$ & & Sensitizer & & & & & REACH (2008-2014) & \\
\hline $\begin{array}{l}\text { 1,5- } \\
\text { Diisocyanatonaphthale } \\
\text { ne }\end{array}$ & $\begin{array}{l}\mathrm{O}=\mathrm{C}=\mathrm{Nc} 1 \mathrm{cccc} 2 \mathrm{c}(\operatorname{cccc} 12 \\
) \mathrm{N}=\mathrm{C}=\mathrm{O}\end{array}$ & & Sensitizer & & & & & REACH (2008-2014) & \\
\hline $\begin{array}{l}\text { 1,6-Bis(2,3- } \\
\text { epoxypropoxy)hexane }\end{array}$ & $\begin{array}{l}\mathrm{C}(\mathrm{CCCOCC} 1 \mathrm{CO} 1) \mathrm{CCOC} \\
\mathrm{C} 1 \mathrm{CO} 1\end{array}$ & & Sensitizer & & & & & ICCVAM (2013) & \\
\hline $\begin{array}{l}\text { 1- }\left(2^{\prime}, 3^{\prime}, 4^{\prime}, 5^{\prime}-\right. \\
\text { Tetramethylphenyl)but } \\
\text { ane-1,3-dione }\end{array}$ & $\begin{array}{l}\mathrm{CC}(\mathrm{O})=\mathrm{CC}(=0) \mathrm{c} 1 \mathrm{cc}(\mathrm{C}) \mathrm{c}( \\
\mathrm{C}) \mathrm{c}(\mathrm{C}) \mathrm{c} 1 \mathrm{C}\end{array}$ & & Sensitizer & & & & & ICCVAM (2013) & \\
\hline $\begin{array}{l}\text { 1-(2',5'- } \\
\text { diethylphenyl)butane- } \\
\text { 1,3-dione }\end{array}$ & $\begin{array}{l}\mathrm{CCc} 1 \mathrm{ccc}(\mathrm{CC}) \mathrm{c}(\mathrm{c} 1) \mathrm{C}(=\mathrm{O}) \\
\mathrm{C}=\mathrm{C}(\mathrm{C}) \mathrm{O}\end{array}$ & & Sensitizer & & & & & ICCVAM (2013) & \\
\hline $\begin{array}{l}\text { 1-(2',5'- } \\
\text { dimethylphenyl)butane } \\
\text {-1,3-dione }\end{array}$ & $\begin{array}{l}\mathrm{CC}(\mathrm{O})=\mathrm{CC}(=0) \mathrm{c} 1 \mathrm{cc}(\mathrm{C}) \mathrm{c} \\
\mathrm{cc} 1 \mathrm{C}\end{array}$ & & Sensitizer & & & & & ICCVAM (2013) & \\
\hline $\begin{array}{l}\text { 1-(2,3-epoxypropoxy)- } \\
\text { 2,2-bis[(2,3- } \\
\text { epoxypropoxy)methylb } \\
\text { utane }\end{array}$ & $\begin{array}{l}\mathrm{CCC}(\mathrm{COCC} 1 \mathrm{CO} 1)(\mathrm{COCC} \\
1 \mathrm{CO} 1) \mathrm{COCC} 1 \mathrm{CO} 1\end{array}$ & & Sensitizer & & & & & ICCVAM (2013) & \\
\hline $\begin{array}{l}\text { 1-(9,9- } \\
\text { dimethyldecylsulfanyl)p } \\
\text { ropan-2-ol }\end{array}$ & $\begin{array}{l}\mathrm{CC}(\mathrm{O}) \operatorname{csccCCCCCCC}(\mathrm{C}) \\
\text { (C)C }\end{array}$ & & Sensitizer & & & & & REACH (2008-2014) & \\
\hline
\end{tabular}




\begin{tabular}{|c|c|c|c|c|c|c|c|c|c|}
\hline Compound name & SMILES & $\begin{array}{l}\text { Human } \\
\text { result }\end{array}$ & $\begin{array}{l}\text { LLNA } \\
\text { result }\end{array}$ & DPRA & KeratinoSens & h-CLAT & $\begin{array}{l}\text { Human } \\
\text { reference }\end{array}$ & LLNA reference & Non-animal reference \\
\hline $\begin{array}{l}\text { 1-Amino-2-nitro-4-bis- } \\
\text { (2-hydroxyethyl)- } \\
\text { amino-benzol }\end{array}$ & $\begin{array}{l}\mathrm{Nc} 1 \operatorname{ccc}(\operatorname{cc} 1 \mathrm{~N}(=\mathrm{O})=0) \mathrm{N}( \\
\mathrm{cCO}) \operatorname{ccO}\end{array}$ & & Sensitizer & & & & & ICCVAM (2013) & \\
\hline 1-Bromodocosane & $\begin{array}{l}\text { CCCCCCCCCCCCCCCCCC } \\
\text { CCCCCBr }\end{array}$ & & Sensitizer & & & & & ICCVAM (2013) & \\
\hline 1-Bromododecane & CCCCCCCCCCCCBr & & Sensitizer & & & & & ICCVAM (2013) & \\
\hline 1-Bromoeicosane & $\begin{array}{l}\text { CCCCCCCCCCCCCCCCCC } \\
\text { CCCBr }\end{array}$ & & Sensitizer & & & & & ICCVAM (2013) & \\
\hline 1-Bromoheptadecane & $\begin{array}{l}\text { CCCCCCCCCCCCCCCCC } \\
\mathrm{Br}\end{array}$ & & Sensitizer & & & & & ICCVAM (2013) & \\
\hline 1-Bromohexadecane & $\begin{array}{l}\text { CCCCCCCCCCCCCCCCB } \\
\mathrm{r}\end{array}$ & & Sensitizer & & & & & ICCVAM (2013) & \\
\hline 1-Bromooctadecane & $\begin{array}{l}\text { CCCCCCCCCCCCCCCCC } \\
\mathrm{CBr}\end{array}$ & & Sensitizer & & & & & ICCVAM (2013) & \\
\hline 1-Bromopentadecane & CCCCCCCCCCCCCCCBr & & Sensitizer & & & & & ICCVAM (2013) & \\
\hline 1-Bromotetradecane & CCCCCCCCCCCCCCBr & & Sensitizer & & & & & ICCVAM (2013) & \\
\hline 1-Bromotridecane & СCCCCCCCCCCCCBr & & Sensitizer & & & & & ICCVAM (2013) & \\
\hline 1-Bromoundecane & CCCCCCCCCCCBr & & Sensitizer & & & & & ICCVAM (2013) & \\
\hline $\begin{array}{l}\text { 1-Chloro-4- } \\
\text { (trifluoromethyl)benzen } \\
\text { e }\end{array}$ & $\mathrm{FC}(\mathrm{F})(\mathrm{F}) \mathrm{c} 1 \mathrm{ccc}(\mathrm{Cl}) \mathrm{cc} 1$ & & Sensitizer & & & & & REACH (2008-2014) & \\
\hline 1-Chlorohexadecane & cCCCCCCCCCCCCCCCCl & & Sensitizer & & & & & ICCVAM (2013) & \\
\hline 1-Chlorooctadecane & $\begin{array}{l}\text { cCCCCCCCCCCCCCCCC } \\
\mathrm{cCl}\end{array}$ & & Sensitizer & & & & & ICCVAM (2013) & \\
\hline 1-Chlorotetradecane & ccCccCccCcCCCCCl & & Sensitizer & & & & & ICCVAM (2013) & \\
\hline 1-lododecane & CCCCCCCCCCCCl & & Sensitizer & & & & & ICCVAM (2013) & \\
\hline 1-Iodononane & CCCCCCCCCI & & Sensitizer & & & & & ICCVAM (2013) & \\
\hline 1-lodotetradecane & сCCCCCCCCCCCCCI & & Sensitizer & & & & & ICCVAM (2013) & \\
\hline $\begin{array}{l}\text { 1-Methyl-3- } \\
\text { nitronitrosoguanidine }\end{array}$ & $\begin{array}{l}\mathrm{CN}(\mathrm{N}=\mathrm{O}) \mathrm{C}(=\mathrm{N}) \mathrm{NN}(=\mathrm{O}) \\
=\mathrm{O}\end{array}$ & & Sensitizer & & & & & ICCVAM (2013) & \\
\hline $\begin{array}{l}\text { 1-Phenyl-2- } \\
\text { methylbutane-1,3- } \\
\text { dione }\end{array}$ & $\begin{array}{l}\mathrm{CC}(\mathrm{O})=\mathrm{C}(\mathrm{C}) \mathrm{C}(=\mathrm{O}) \mathrm{c} 1 \mathrm{ccc} \\
\mathrm{cc1}\end{array}$ & & Sensitizer & & & & & ICCVAM (2013) & \\
\hline $\begin{array}{l}\text { 1-Phenyloctane-1,3- } \\
\text { dione }\end{array}$ & $\begin{array}{l}\operatorname{cccccc}(0)=\operatorname{CC}(=0) \operatorname{c1c} \\
\operatorname{cccc} 1\end{array}$ & & Sensitizer & & & & & ICCVAM (2013) & \\
\hline $\begin{array}{l}\text { 1-[2-(2-prop-2- } \\
\text { enoyloxypropoxy)propo } \\
\text { xy]propan-2-yl prop-2- } \\
\text { enoate }\end{array}$ & $\begin{array}{l}\mathrm{CC}(\operatorname{COC}(\mathrm{C}) \operatorname{COC}(=0) \mathrm{C}=\mathrm{C} \\
\operatorname{lOCC}(\mathrm{C}) \mathrm{OC}(=0) \mathrm{C}=\mathrm{C}\end{array}$ & & Sensitizer & & & & & REACH (2008-2014) & \\
\hline
\end{tabular}




\begin{tabular}{|c|c|c|c|c|c|c|c|c|c|}
\hline Compound name & SMILES & $\begin{array}{l}\text { Human } \\
\text { result }\end{array}$ & $\begin{array}{l}\text { LLNA } \\
\text { result }\end{array}$ & DPRA & KeratinoSens & h-CLAT & $\begin{array}{l}\text { Human } \\
\text { reference }\end{array}$ & LLNA reference & Non-animal reference \\
\hline $\begin{array}{l}\text { 1-aminocyclopropane- } \\
\text { 1-carbonitrile }\end{array}$ & NC1(CC1)C\#N & & Sensitizer & & & & & REACH (2008-2014) & \\
\hline $\begin{array}{l}\text { 1-butoxy-3- } \\
\text { phenoxypropan-2-ol }\end{array}$ & $\begin{array}{l}\text { CCCCOCC }(0) \operatorname{COc} 1 \mathrm{ccccc} \\
1\end{array}$ & & Sensitizer & & & & & REACH (2008-2014) & \\
\hline 1-ethenoxyoctadecane & $\begin{array}{l}\text { CCCCCCCCCCCCCCCCC } \\
\mathrm{COC}=\mathrm{C}\end{array}$ & & Sensitizer & & & & & REACH (2008-2014) & \\
\hline 1-ethenylazepan-2-one & $\mathrm{C}=\mathrm{CN} 1 \mathrm{CCCCCC} 1=\mathrm{O}$ & & Sensitizer & & & & & REACH (2008-2014) & \\
\hline $\begin{array}{l}\text { 1-methyl-4-propan-2- } \\
\text { ylidenecyclohexene }\end{array}$ & $\mathrm{CC}(\mathrm{C})=\mathrm{C} 1 \mathrm{CCC}(\mathrm{C})=\mathrm{CC} 1$ & & Sensitizer & & & & & REACH (2008-2014) & \\
\hline $\begin{array}{l}\text { 1-morpholin-4- } \\
\text { ylethanone }\end{array}$ & $\mathrm{CC}(=0) \mathrm{N} 1 \mathrm{CCOCC} 1$ & & Sensitizer & & & & & REACH (2008-2014) & \\
\hline $\begin{array}{l}\text { 12-Bromododecanoic } \\
\text { acid }\end{array}$ & $\begin{array}{l}\mathrm{OC}(=\mathrm{O}) \mathrm{CCCCCCCCCCCB} \\
\mathrm{r}\end{array}$ & & Sensitizer & & & & & ICCVAM (2013) & \\
\hline $\begin{array}{l}\text { 14-Hydroxynor- } \\
\text { morphinone }\end{array}$ & $\begin{array}{l}\text { Oc1ccc2CC3NCCC45C( } \\
\text { Oc1c24)C }(=0) \mathrm{C}=\mathrm{CC} 35 \\
\mathrm{O}\end{array}$ & & Sensitizer & & & & & ICCVAM (2013) & \\
\hline $\begin{array}{l}\text { 2',3',4'- } \\
\text { Trihydroxychalcone }\end{array}$ & $\begin{array}{l}\operatorname{Oc} 1 \mathrm{ccc}(\mathrm{C}(=\mathrm{O}) \mathrm{C}=\mathrm{Cc} 2 \mathrm{ccc} \\
\operatorname{cc} 2) \mathrm{c}(\mathrm{O}) \mathrm{c} 10\end{array}$ & & Sensitizer & & & & & ICCVAM (2013) & \\
\hline 2'-4'-dihydroxychalcone & $\begin{array}{l}\mathrm{Oc} 1 \mathrm{ccc}(\mathrm{C}(=\mathrm{O}) \mathrm{C}=\mathrm{Cc} 2 \mathrm{ccc} \\
\operatorname{cc} 2) \mathrm{c}(\mathrm{O}) \mathrm{c} 1\end{array}$ & & Sensitizer & & & & & ICCVAM (2013) & \\
\hline $\begin{array}{l}\text { 2,2-dimethyl-3- } \\
\text { (morpholin-4- } \\
\text { yl)propanal }\end{array}$ & $\begin{array}{l}\mathrm{CC}(\mathrm{C})(\mathrm{CN} 1 \mathrm{CCOCC} 1) \mathrm{C}= \\
\mathrm{O}\end{array}$ & & Sensitizer & & & & & REACH (2008-2014) & \\
\hline $\begin{array}{l}2,3,5,6- \\
\text { tetrachlorocyclohexa- } \\
\text { 2,5-diene-1,4-dione }\end{array}$ & $\begin{array}{l}\mathrm{ClC} 1=\mathrm{C}(\mathrm{Cl}) \mathrm{C}(=0) \mathrm{C}(\mathrm{Cl})= \\
\mathrm{C}(\mathrm{Cl}) \mathrm{C} 1=\mathrm{O}\end{array}$ & & Sensitizer & & & & & REACH (2008-2014) & \\
\hline 2,3,6-Trimethylphenol & $\operatorname{Cc} 1 \mathrm{ccc}(\mathrm{C}) \mathrm{c}(\mathrm{O}) \mathrm{c} 1 \mathrm{C}$ & & Sensitizer & & & & & REACH (2008-2014) & \\
\hline $\begin{array}{l}\text { 2,4,4-trimethylpentan- } \\
2 \text {-yl 2- } \\
\text { ethylhexaneperoxoate }\end{array}$ & $\begin{array}{l}\mathrm{CCCCC}(\mathrm{CC}) \mathrm{C}(=\mathrm{O}) \mathrm{OOC}(\mathrm{C} \\
)(\mathrm{C}) \mathrm{CC}(\mathrm{C})(\mathrm{C}) \mathrm{C}\end{array}$ & & Sensitizer & & & & & REACH (2008-2014) & \\
\hline $\begin{array}{l}2,4,5- \\
\text { Trichlorophenoxyacetic } \\
\text { Acid }\end{array}$ & $\begin{array}{l}\mathrm{OC}(=0) \operatorname{coc} 1 \mathrm{cc}(\mathrm{Cl}) \mathrm{c}(\mathrm{Cl}) \\
\mathrm{cc} 1 \mathrm{Cl}\end{array}$ & & Sensitizer & & & & & ICCVAM (2013) & \\
\hline $\begin{array}{l}\text { 2,4,6-Trichloro-1,3,5- } \\
\text { triazine }\end{array}$ & Clc1nc(Cl)nc(Cl)n1 & & Sensitizer & & & & & ICCVAM (2013) & \\
\hline 2,4-Dichloropyrimidine & $\operatorname{Clc} 1 \mathrm{ccnc}(\mathrm{Cl}) \mathrm{n} 1$ & & Sensitizer & & & & & ICCVAM (2013) & \\
\hline 2,4-Hexadienal & $\mathrm{CC}=\mathrm{CC}=\mathrm{CC}=\mathrm{O}$ & & Sensitizer & & & & & ICCVAM (2013) & \\
\hline 2,4-di(ethenyl)phenol & $\operatorname{Oc} 1 \mathrm{ccc}(\mathrm{C}=\mathrm{C}) \operatorname{cc} 1 \mathrm{C}=\mathrm{C}$ & & Sensitizer & & & & & REACH (2008-2014) & \\
\hline $\begin{array}{l}\text { 2,6-Dimethoxy-4- } \\
\text { methyl-8-nitro-5-[3- } \\
\text { (trifluoromethyl)- } \\
\text { phenoxy]quinoline }\end{array}$ & $\begin{array}{l}\operatorname{COc} 1 c c(C) c 2 c(O c 3 c c c c( \\
c 3) C(F)(F) F) c(O C) c c(c 2 \\
n 1) N(=O)=0\end{array}$ & & Sensitizer & & & & & ICCVAM (2013) & \\
\hline
\end{tabular}




\begin{tabular}{|c|c|c|c|c|c|c|c|c|c|}
\hline Compound name & SMILES & $\begin{array}{l}\text { Human } \\
\text { result }\end{array}$ & $\begin{array}{l}\text { LLNA } \\
\text { result }\end{array}$ & DPRA & KeratinoSens & h-CLAT & $\begin{array}{l}\text { Human } \\
\text { reference }\end{array}$ & LLNA reference & Non-animal reference \\
\hline $\begin{array}{l}\text { 2,6-ditert-butyl-4- } \\
\text { (dimethylaminomethyl) } \\
\text { phenol }\end{array}$ & $\begin{array}{l}\mathrm{CN}(\mathrm{C}) \mathrm{Cc} 1 \mathrm{cc}(\mathrm{c}(\mathrm{O}) \mathrm{c}(\mathrm{c} 1) \mathrm{C} \\
(\mathrm{C})(\mathrm{C}) \mathrm{C}) \mathrm{C}(\mathrm{C})(\mathrm{C}) \mathrm{C}\end{array}$ & & Sensitizer & & & & & REACH (2008-2014) & \\
\hline $\begin{array}{l}2,7,7- \\
\text { trimethylbicyclo[3.1.1]h } \\
\text { eptane-3-carbaldehyde }\end{array}$ & $\begin{array}{l}\mathrm{CC} 1 \mathrm{C} 2 \mathrm{CC}(\mathrm{CC} 1 \mathrm{C}=0) \mathrm{C} 2( \\
\mathrm{C}) \mathrm{C}\end{array}$ & & Sensitizer & & & & & REACH (2008-2014) & \\
\hline $\begin{array}{l}\text { 2-(2,4- } \\
\text { Diaminophenoxy)ethan } \\
\text { ol dihydrochloride }\end{array}$ & $\mathrm{Nc} 1 \mathrm{ccc}(\mathrm{OCCO}) \mathrm{c}(\mathrm{N}) \mathrm{c} 1$ & & Sensitizer & & & & & $\begin{array}{l}\text { ICCVAM (2013)+ } \\
\text { REACH (2008-2014) }\end{array}$ & \\
\hline $\begin{array}{l}\text { 2-(2-propan-2-yl-1,3- } \\
\text { oxazolidin-3-yl)ethanol }\end{array}$ & $\mathrm{CC}(\mathrm{C}) \mathrm{C} 10 \mathrm{CCN} 1 \mathrm{CCO}$ & & Sensitizer & & & & & REACH (2008-2014) & \\
\hline $\begin{array}{l}\text { 2-(2-propan-2-yl-1,3- } \\
\text { oxazolidin-3-yl)ethyl N- } \\
\text { [6-[2-(2-propan-2-yl- } \\
\text { 1,3-oxazolidin-3- } \\
\text { yl)ethoxycarbonylamin } \\
\text { o]hexyl]carbamate }\end{array}$ & $\begin{array}{l}\text { CC(C)C1OCCN1CCOC(= } \\
\text { O)NCCCCCCNC(=O)OC } \\
\text { CN1CCOC1C(C)C }\end{array}$ & & Sensitizer & & & & & REACH (2008-2014) & \\
\hline $\begin{array}{l}\text { 2-(3,4-dimethyl- } \\
\text { phenyl)-5-methyl-2,4- } \\
\text { dihydropyrazol-3-one }\end{array}$ & $\begin{array}{l}\mathrm{CC} 1=\mathrm{CC}(=0) \mathrm{N}(\mathrm{N} 1) \mathrm{c} 1 \mathrm{cc} \\
\mathrm{c}(\mathrm{C}) \mathrm{c}(\mathrm{C}) \mathrm{c} 1\end{array}$ & & Sensitizer & & & & & ICCVAM (2013) & \\
\hline $\begin{array}{l}\text { 2-(4-Amino-2nitro- } \\
\text { phenylamino)-ethanol }\end{array}$ & $\begin{array}{l}\mathrm{Nc1} 1 \mathrm{ccc}(\mathrm{NCCO}) \mathrm{c}(\mathrm{c} 1) \mathrm{N}(= \\
\mathrm{O})=\mathrm{O}\end{array}$ & & Sensitizer & & & & & ICCVAM (2013) & \\
\hline $\begin{array}{l}\text { 2-(4-tert- } \\
\text { Amylcyclohexyl)acetald } \\
\text { ehyde }\end{array}$ & $\begin{array}{l}\operatorname{CCC}(\mathrm{C})(\mathrm{C}) \mathrm{C} 1 \mathrm{CCC}(\mathrm{CC}=0) \\
\mathrm{CC} 1\end{array}$ & & Sensitizer & & & & & ICCVAM (2013) & \\
\hline $\begin{array}{l}\text { 2-(benzotriazol-2-yl)-4- } \\
\text { methylphenol }\end{array}$ & $\begin{array}{l}\mathrm{Cc} 1 \mathrm{ccc}(\mathrm{O}) \mathrm{c}(\mathrm{c} 1)- \\
\mathrm{n} 1 \mathrm{nc} 2 \mathrm{ccccc} 2 \mathrm{n} 1\end{array}$ & & Sensitizer & & & & & REACH (2008-2014) & \\
\hline $\begin{array}{l}2- \\
\text { (chloromethyl)oxirane; } \\
\text { formaldehyde; phenol }\end{array}$ & Oc1ccccc1 & & Sensitizer & & & & & REACH (2008-2014) & \\
\hline $\begin{array}{l}\text { 2- } \\
\text { (hexadecyloxy)ethanol }\end{array}$ & $\begin{array}{l}\text { CCCCCCCCCCCCCCCCO } \\
\mathrm{cCO}\end{array}$ & & Sensitizer & & & & & ICCVAM (2013) & \\
\hline $\begin{array}{l}\text { 2-(\{3- } \\
\text { aminopyrazolo[1,5- } \\
\text { a]pyridin-2- } \\
\text { yl\}oxy)ethan-1-ol }\end{array}$ & $\begin{array}{l}\text { Nc1c(OCCO)nn2cccec1 } \\
2\end{array}$ & & Sensitizer & & & & & REACH (2008-2014) & \\
\hline $\begin{array}{l}\text { 2-Amino-6-chloro-4- } \\
\text { nitrophenol }\end{array}$ & $\begin{array}{l}\mathrm{Nc} 1 \mathrm{cc}(\mathrm{cc}(\mathrm{Cl}) \mathrm{c1O}) \mathrm{N}(=\mathrm{O}) \\
=0\end{array}$ & & Sensitizer & & & & & ICCVAM (2013) & \\
\hline $\begin{array}{l}\text { 2-Amino-di- } \\
\text { phenylamine }\end{array}$ & Nc1ccccc1Nc1ccccc1 & & Sensitizer & & & & & ICCVAM (2013) & \\
\hline $\begin{array}{l}\text { 2-Benzyl-tert- } \\
\text { butylamino-3'- } \\
\text { hydroxymethyl-4'- } \\
\text { hydroxyaceto-phenone } \\
\text { hydrochloride }\end{array}$ & $\begin{array}{l}\mathrm{CC}(\mathrm{C})(\mathrm{C}) \mathrm{N}(\mathrm{CC}(=\mathrm{O}) \mathrm{c} 1 \mathrm{ccc} \\
(\mathrm{O}) \mathrm{c}(\mathrm{CO}) \mathrm{c} 1) \mathrm{Cc} 1 \mathrm{ccccc} 1\end{array}$ & & Sensitizer & & & & & ICCVAM (2013) & \\
\hline
\end{tabular}




\begin{tabular}{|c|c|c|c|c|c|c|c|c|c|}
\hline Compound name & SMILES & $\begin{array}{l}\text { Human } \\
\text { result }\end{array}$ & $\begin{array}{l}\text { LLNA } \\
\text { result }\end{array}$ & DPRA & KeratinoSens & h-CLAT & $\begin{array}{l}\text { Human } \\
\text { reference }\end{array}$ & LLNA reference & Non-animal reference \\
\hline $\begin{array}{l}\text { 2-Bromo-5-hydroxy- } \\
\text { benzaldehyde }\end{array}$ & Oc1ccc $(\mathrm{Br}) \mathrm{c}(\mathrm{C}=\mathrm{O}) \mathrm{c1}$ & & Sensitizer & & & & & ICCVAM (2013) & \\
\hline $\begin{array}{l}\text { 2-Bromotetradecanoic } \\
\text { acid }\end{array}$ & $\begin{array}{l}\operatorname{ccccccccccccC}(\mathrm{Br}) \mathrm{Cl} \\
\mathrm{0})=0\end{array}$ & & Sensitizer & & & & & ICCVAM (2013) & \\
\hline $\begin{array}{l}\text { 2-Chloro-1-[(3- } \\
\text { Fluorophenyl)- } \\
\text { Methoxy]-4- } \\
\text { Nitrobenzene }\end{array}$ & $\begin{array}{l}\mathrm{Fc} 1 \mathrm{cccc}(\mathrm{COc} 2 \mathrm{ccc}(\mathrm{cc} 2 \mathrm{Cl} \\
\mathrm{N}(=0)=0) \mathrm{c} 1\end{array}$ & & Sensitizer & & & & & ICCVAM (2013) & \\
\hline $\begin{array}{l}\text { 2-Ethylhexyl diphenyl } \\
\text { phosphite }\end{array}$ & $\begin{array}{l}\text { ccCCC(CC)COP(Oc1ccc } \\
\text { cc1)Oc1ccccc1 }\end{array}$ & & Sensitizer & & & & & REACH (2008-2014) & \\
\hline $\begin{array}{l}\text { 2-Methyl-4,5- } \\
\text { trimethylene-4- } \\
\text { isothiazolin-3-one }\end{array}$ & $\mathrm{CN} 1 \mathrm{SC} 2=\mathrm{C}(\mathrm{CCC} 2) \mathrm{C} 1=\mathrm{O}$ & & Sensitizer & & & & & ICCVAM (2013) & \\
\hline $\begin{array}{l}\text { 2-Methyl-5- } \\
\text { hydroxyethylaminophe } \\
\text { nol }\end{array}$ & Cc1ccc(NCCO)cc10 & & Sensitizer & & & & & ICCVAM (2013) & \\
\hline $\begin{array}{l}\text { 2-Methyl-5- } \\
\text { nitrobenzenesulfonic } \\
\text { acid }\end{array}$ & $\begin{array}{l}\operatorname{Cc} 1 \operatorname{ccc}(\operatorname{cc} 1 S(O)(=0)=0) \\
N(=0)=0\end{array}$ & & Sensitizer & & & & & REACH (2008-2014) & \\
\hline 2-Methylpentanal & $\operatorname{CCCC}(\mathrm{C}) \mathrm{C}=\mathrm{O}$ & & Sensitizer & & & & & REACH (2008-2014) & \\
\hline 2-Methylresorcinol & $\operatorname{cc} 1 \mathrm{c}(0) \operatorname{cccc} 10$ & & Sensitizer & & & & & REACH (2008-2014) & \\
\hline $\begin{array}{l}\text { 2-Nitro-4- } \\
\text { (propylthio)aniline }\end{array}$ & $\begin{array}{l}\operatorname{CCCSc} 1 \operatorname{ccc}(N) c(c 1) N(= \\
0)=0\end{array}$ & & Sensitizer & & & & & ICCVAM (2013) & \\
\hline $\begin{array}{l}\text { 2-[1-(3,3- } \\
\text { dimethylcyclohexyl)eth } \\
\text { oxy]-2-methylpropyl } \\
\text { cyclopropanecarboxylat } \\
\text { e }\end{array}$ & $\begin{array}{l}\mathrm{CC}(\mathrm{OC}(\mathrm{C})(\mathrm{C}) \mathrm{COC}(=\mathrm{O}) \mathrm{C} 1 \\
\mathrm{CC} 1) \mathrm{C} 1 \mathrm{CCC}(\mathrm{C})(\mathrm{C}) \mathrm{C} 1\end{array}$ & & Sensitizer & & & & & REACH (2008-2014) & \\
\hline $\begin{array}{l}\text { 2-[1-(4-Bromophenyl)- } \\
\text { 1-phenylethoxy]-N,N- } \\
\text { dimethylethanamine } \\
\text { hydrochloride }\end{array}$ & $\begin{array}{l}\mathrm{CN}(\mathrm{C}) \mathrm{CCOC}(\mathrm{C})(\mathrm{c} 1 \mathrm{ccccc} \\
1) \mathrm{c} 1 \mathrm{ccc}(\mathrm{Br}) \mathrm{cc} 1\end{array}$ & & Sensitizer & & & & & ICCVAM (2013) & \\
\hline $\begin{array}{l}\text { 2-[2,2-bis(2-prop-2- } \\
\text { enoyloxyethoxymethyl) } \\
\text { butoxy]ethyl prop-2- } \\
\text { enoate }\end{array}$ & $\begin{array}{l}\operatorname{ccc}(\operatorname{coccoc}(=0) C=C)( \\
\operatorname{coc} C O C(=O) C=C) \operatorname{cocc} \\
O C(=O) C=C\end{array}$ & & Sensitizer & & & & & REACH (2008-2014) & \\
\hline $\begin{array}{l}\text { 2-[2-[3,4-bis(2- } \\
\text { hydroxyethoxy)oxolan- } \\
\text { 2-yl]-2-(2- } \\
\text { hydroxyethoxy)ethoxy] } \\
\text { ethyl dodecanoate }\end{array}$ & $\begin{array}{l}\text { CCCCCCCCCCCC }(=0) O C \\
\text { COCC }(\text { OCCO)C1OCC(O } \\
\text { CCO)C1OCCO }\end{array}$ & & Sensitizer & & & & & REACH (2008-2014) & \\
\hline $\begin{array}{l}\text { 2-[4-(2- } \\
\text { hydroxyethoxy)but-2- } \\
\text { ynoxy]ethanol }\end{array}$ & OCCOCC\#CCOCCO & & Sensitizer & & & & & REACH (2008-2014) & \\
\hline
\end{tabular}




\begin{tabular}{|c|c|c|c|c|c|c|c|c|c|}
\hline Compound name & SMILES & $\begin{array}{l}\text { Human } \\
\text { result }\end{array}$ & $\begin{array}{l}\text { LLNA } \\
\text { result }\end{array}$ & DPRA & KeratinoSens & h-CLAT & $\begin{array}{l}\text { Human } \\
\text { reference }\end{array}$ & LLNA reference & Non-animal reference \\
\hline $\begin{array}{l}\text { 2-cyanoethyl-[6-(2- } \\
\text { cyanoethylazaniumyl)h } \\
\text { exyl]azanium }\end{array}$ & $\begin{array}{l}\text { N\#CCCNCCCCCCNCCC\# } \\
\mathrm{N}\end{array}$ & & Sensitizer & & & & & REACH (2008-2014) & \\
\hline $\begin{array}{l}2- \\
\text { cyclohexylsulfanylisoind } \\
\text { ole-1,3-dione }\end{array}$ & $\begin{array}{l}\mathrm{O}=\mathrm{C} 1 \mathrm{~N}(\mathrm{SC} 2 \mathrm{CCCCC} 2) \mathrm{C}( \\
=0) \operatorname{coc} \operatorname{cccc} 12\end{array}$ & & Sensitizer & & & & & REACH (2008-2014) & \\
\hline $\begin{array}{l}\text { 2-ethyl-4-methyl-3H- } \\
\text { imidazole }\end{array}$ & CCc1ncc(C)[nH]1 & & Sensitizer & & & & & REACH (2008-2014) & \\
\hline $\begin{array}{l}\text { 2-ethylhexyl 2-[2-(2- } \\
\text { ethylhexoxy)-2- } \\
\text { oxoethyl]sulfanylacetat } \\
\text { e }\end{array}$ & $\begin{array}{l}\text { CCCCC }(C C) \operatorname{COC}(=0) \operatorname{CSC} \\
\mathrm{C}(=0) \mathrm{OCC}(\mathrm{CC}) \mathrm{CCCC}\end{array}$ & & Sensitizer & & & & & REACH (2008-2014) & \\
\hline $\begin{array}{l}\text { 2-hydroxyethyl 2- } \\
\text { methylprop-2-enoate; } \\
\text { phosphoric acid }\end{array}$ & $\mathrm{CC}(=\mathrm{C}) \mathrm{C}(=\mathrm{O}) \mathrm{OCCO}$ & & Sensitizer & & & & & REACH (2008-2014) & \\
\hline $\begin{array}{l}\text { 2-methoxyethyl prop-2- } \\
\text { enoate }\end{array}$ & $\operatorname{coc} \operatorname{coc}(=0) C=C$ & & Sensitizer & & & & & REACH (2008-2014) & \\
\hline $\begin{array}{l}\text { 2-methyloctane-1,8- } \\
\text { diamine }\end{array}$ & $\mathrm{CC}(\mathrm{CN}) \mathrm{CCCCCCN}$ & & Sensitizer & & & & & REACH (2008-2014) & \\
\hline $\begin{array}{l}\text { 2-methylprop-2-enoyl } \\
\text { 2-methylprop-2-enoate }\end{array}$ & $\begin{array}{l}\mathrm{CC}(=\mathrm{C}) \mathrm{C}(=\mathrm{O}) \mathrm{OC}(=\mathrm{O}) \mathrm{C}(\mathrm{C} \\
)=\mathrm{C}\end{array}$ & & Sensitizer & & & & & REACH (2008-2014) & \\
\hline $\begin{array}{l}\text { 2-\{4-chloro-3-[(4- } \\
\text { ethoxyphenyl)methyl]p } \\
\text { henyl\}-6- } \\
\text { (hydroxymethyl)-2- } \\
\text { methoxyoxane-3,4,5- } \\
\text { triol }\end{array}$ & $\begin{array}{l}\mathrm{CCOc} 1 \mathrm{ccc}(\mathrm{Cc} 2 \mathrm{cc}(\mathrm{ccc} 2 \mathrm{Cl} \\
) \mathrm{C2}(\mathrm{OC}) \mathrm{OC}(\mathrm{CO}) \mathrm{C}(\mathrm{O}) \mathrm{Cl} \\
\mathrm{O}) \mathrm{C2O}) \mathrm{cc} 1\end{array}$ & & Sensitizer & & & & & REACH (2008-2014) & \\
\hline $\begin{array}{l}\text { 2-\{6,6- } \\
\text { dimethylbicyclo[3.1.1]h } \\
\text { ept-2-en-2-yl\}ethyl } \\
\text { acetate }\end{array}$ & $\begin{array}{l}\mathrm{CC}(=0) O C C C 1=C C C 2 C C \\
1 \mathrm{C} 2(\mathrm{C}) \mathrm{C}\end{array}$ & & Sensitizer & & & & & REACH (2008-2014) & \\
\hline $\begin{array}{l}\text { 2-\{[2-(4- } \\
\text { aminophenyl)ethyl]ami } \\
\text { no\}-1-phenylethan-1-ol }\end{array}$ & $\begin{array}{l}\text { Nc1 } 1 \mathrm{ccc}(\mathrm{CCNCC}(\mathrm{O}) \mathrm{c} 2 \mathrm{ccc} \\
\mathrm{cc} 2) \mathrm{cc} 1\end{array}$ & & Sensitizer & & & & & REACH (2008-2014) & \\
\hline $\begin{array}{l}\text { 3,4-Dichloroaniline } \\
\text { hydrochloride }\end{array}$ & $\operatorname{Nc1ccc}(\mathrm{Cl}) c(\mathrm{Cl}) \mathrm{c1}$ & & Sensitizer & & & & & ICCVAM (2013) & \\
\hline 3,4-Dihydro-2H-pyran & $\mathrm{C} 1 \mathrm{COC}=\mathrm{CC} 1$ & & Sensitizer & & & & & REACH (2008-2014) & \\
\hline 3,4-Dinitrophenol & $\begin{array}{l}\text { Oc1ccc(c(c1)N(=O)=O) } \\
\mathrm{N}(=\mathrm{O})=\mathrm{O}\end{array}$ & & Sensitizer & & & & & ICCVAM (2013) & \\
\hline $\begin{array}{l}3,5,5- \\
\text { Trimethylhexanoyl } \\
\text { chloride }\end{array}$ & $\mathrm{CC}(\mathrm{CC}(\mathrm{Cl})=\mathrm{O}) \mathrm{CC}(\mathrm{C})(\mathrm{C}) \mathrm{C}$ & & Sensitizer & & & & & ICCVAM (2013) & \\
\hline
\end{tabular}




\begin{tabular}{|c|c|c|c|c|c|c|c|c|c|}
\hline Compound name & SMILES & $\begin{array}{l}\text { Human } \\
\text { result }\end{array}$ & $\begin{array}{l}\text { LLNA } \\
\text { result }\end{array}$ & DPRA & KeratinoSens & h-CLAT & $\begin{array}{l}\text { Human } \\
\text { reference }\end{array}$ & LLNA reference & Non-animal reference \\
\hline $\begin{array}{l}\text { 3,5-Diamino-2,6- } \\
\text { dimethoxypyridinedihy } \\
\text { drochloride }\end{array}$ & $\operatorname{coc} 1 \mathrm{nc}(\mathrm{OC}) \mathrm{c}(\mathrm{N}) \mathrm{cc} 1 \mathrm{~N}$ & & Sensitizer & & & & & ICCVAM (2013) & \\
\hline $\begin{array}{l}\text { 3- } \\
\text { (Chloromethyl)heptane }\end{array}$ & $\mathrm{CCCCC}(\mathrm{CC}) \mathrm{CCl}$ & & Sensitizer & & & & & REACH (2008-2014) & \\
\hline $\begin{array}{l}\text { 3- } \\
\text { (dodecanoylamino)prop } \\
\text { yl-trimethylazanium; } \\
\text { methyl sulfate }\end{array}$ & $\begin{array}{l}\mathrm{CCCCCCCCCCCC}(=0) N C \\
\mathrm{CC}[\mathrm{N}+](\mathrm{C})(\mathrm{C}) \mathrm{C}\end{array}$ & & Sensitizer & & & & & REACH (2008-2014) & \\
\hline $\begin{array}{l}\text { 3-(ethenyloxy)propan- } \\
\text { 1-amine }\end{array}$ & $\mathrm{NCCCOC}=\mathrm{C}$ & & Sensitizer & & & & & REACH (2008-2014) & \\
\hline $\begin{array}{l}\text { 3-(pentadec-8-en-1- } \\
\text { yl)phenol }\end{array}$ & $\begin{array}{l}\mathrm{cCCCCCCC}=\operatorname{CCCCCCCCc} \\
1 \operatorname{cccc}(0) \mathrm{c} 1\end{array}$ & & Sensitizer & & & & & REACH (2008-2014) & \\
\hline $\begin{array}{l}\text { 3- } \\
\text { (tridecylcarbamoyl)pro } \\
\text { p-2-enoic acid }\end{array}$ & $\begin{array}{l}\text { CCCCCCCCCCCCCNC(= } \\
\text { O) } \mathrm{C}=\mathrm{CC}(\mathrm{O})=\mathrm{O}\end{array}$ & & Sensitizer & & & & & REACH (2008-2014) & \\
\hline $\begin{array}{l}\text { 3-Amino-5-mercapto- } \\
\text { 1,2,4-triazole }\end{array}$ & $\mathrm{NC} 1=\mathrm{NNC}(=\mathrm{S}) \mathrm{N} 1$ & & Sensitizer & & & & & ICCVAM (2013) & \\
\hline $\begin{array}{l}\text { 3-Aminomethyl-3,5,5- } \\
\text { trimethylcyclohexylami } \\
\text { ne }\end{array}$ & $\begin{array}{l}\mathrm{CC} 1(\mathrm{C}) \mathrm{CC}(\mathrm{N}) \mathrm{CC}(\mathrm{C})(\mathrm{CN}) \\
\mathrm{C} 1\end{array}$ & & Sensitizer & & & & & ICCVAM (2013) & \\
\hline $\begin{array}{l}\text { 3-Bromomethyl-5,5- } \\
\text { dimethyl-dihydro- } \\
\text { 2(3H)-furanone }\end{array}$ & $\mathrm{CC} 1(\mathrm{C}) \mathrm{CC}(\mathrm{CBr}) \mathrm{C}(=\mathrm{O}) \mathrm{O} 1$ & & Sensitizer & & & & & ICCVAM (2013) & \\
\hline $\begin{array}{l}\text { 3-Chloro-4- } \\
\text { fluorobenzoyl chloride }\end{array}$ & $\mathrm{Fc} 1 \mathrm{ccc}(\mathrm{cc} 1 \mathrm{Cl}) \mathrm{C}(\mathrm{Cl})=0$ & & Sensitizer & & & & & ICCVAM (2013) & \\
\hline $\begin{array}{l}\text { 3-Ethoxy-1-(2',3',4',5'- } \\
\text { tetramethylphenyl)pro } \\
\text { pane-1,3-dione }\end{array}$ & $\begin{array}{l}\mathrm{cCOC}(=0) \mathrm{CC}(=0) \mathrm{c} 1 \mathrm{cc}( \\
\mathrm{C}) \mathrm{c}(\mathrm{C}) \mathrm{c}(\mathrm{C}) \mathrm{c} 1 \mathrm{C}\end{array}$ & & Sensitizer & & & & & ICCVAM (2013) & \\
\hline $\begin{array}{l}\text { 3-Fluoro-5-(3- } \\
\text { pyridinyl)benzen-amine }\end{array}$ & $\begin{array}{l}\text { Nc1cc(F)cc(c1)- } \\
\text { c1cccnc1 }\end{array}$ & & Sensitizer & & & & & ICCVAM (2013) & \\
\hline $\begin{array}{l}\text { 3-Methyl-4-phenyl- } \\
\text { 1,2,5-thiadiazole-1,1- } \\
\text { dioxide }\end{array}$ & $\begin{array}{l}\mathrm{C}=\mathrm{C} 1 \mathrm{NS}(=0)(=0) \mathrm{N}=\mathrm{C} 1 \mathrm{c} \\
1 \operatorname{ccccc} 1\end{array}$ & & Sensitizer & & & & & ICCVAM (2013) & \\
\hline 3-Methyleugenol & $\operatorname{coc} 1 \mathrm{c}(\mathrm{C}) \mathrm{c}(\mathrm{CC}=\mathrm{C}) \mathrm{ccc} 10$ & & Sensitizer & & & & & ICCVAM (2013) & \\
\hline 3-Methylisoeugenol & $\operatorname{COc} 1 c(C) c(C=C C) \operatorname{ccc} 10$ & & Sensitizer & & & & & ICCVAM (2013) & \\
\hline 3-Phenylenediamine & $\operatorname{Nc1} \operatorname{cccc}(\mathrm{N}) \mathrm{c1}$ & & Sensitizer & & & & & $\begin{array}{l}\text { ICCVAM (2013) + } \\
\text { REACH }(2008-2014)\end{array}$ & \\
\hline $\begin{array}{l}\text { 3-[2,2-dimethyl-3-(3- } \\
\text { prop-2- } \\
\text { enoyloxypropoxy)propo } \\
\text { xy]propyl prop-2- } \\
\text { enoate }\end{array}$ & $\begin{array}{l}\mathrm{CC}(\mathrm{C})(\operatorname{cocccoc}(=0) \mathrm{C}= \\
\mathrm{C}) \operatorname{cocccoc}(=0) \mathrm{C}=\mathrm{C}\end{array}$ & & Sensitizer & & & & & REACH (2008-2014) & \\
\hline
\end{tabular}




\begin{tabular}{|c|c|c|c|c|c|c|c|c|c|}
\hline Compound name & SMILES & $\begin{array}{l}\text { Human } \\
\text { result }\end{array}$ & $\begin{array}{l}\text { LLNA } \\
\text { result }\end{array}$ & DPRA & KeratinoSens & h-CLAT & $\begin{array}{l}\text { Human } \\
\text { reference }\end{array}$ & LLNA reference & Non-animal reference \\
\hline $\begin{array}{l}\text { 3-[2,3-bis(3-prop-2- } \\
\text { enoyloxypropoxy)propo } \\
\text { xy]propyl prop-2- } \\
\text { enoate }\end{array}$ & $\begin{array}{l}\mathrm{C}=\mathrm{CC}(=\mathrm{O}) \mathrm{OCCCOCC}(\mathrm{CO} \\
\mathrm{CCCOC}(=\mathrm{O}) \mathrm{C}=\mathrm{C}) \mathrm{OCCCO} \\
\mathrm{C}(=\mathrm{O}) \mathrm{C}=\mathrm{C}\end{array}$ & & Sensitizer & & & & & REACH (2008-2014) & \\
\hline $\begin{array}{l}\text { 3-[2-[bis[2-(3- } \\
\text { hydroxypropoxy)ethyl]a } \\
\text { mino]ethoxy]propan-1- } \\
\text { ol }\end{array}$ & $\begin{array}{l}\text { OCCCOCCN }(\mathrm{CCOCCCO}) \\
\text { CCOCCCO }\end{array}$ & & Sensitizer & & & & & REACH (2008-2014) & \\
\hline 3-aminooctan-4-ol & $\operatorname{CCCCC}(\mathrm{O}) \mathrm{C}(\mathrm{N}) \mathrm{CC}$ & & Sensitizer & & & & & REACH (2008-2014) & \\
\hline $\begin{array}{l}\text { 3-methyl-1-(3- } \\
\text { methylbutoxy)butane }\end{array}$ & $\mathrm{CC}(\mathrm{C}) \mathrm{CCOCCC}(\mathrm{C}) \mathrm{C}$ & & Sensitizer & & & & & REACH (2008-2014) & \\
\hline $\begin{array}{l}\text { 3-methylcyclotetradec- } \\
\text { 5-en-1-one }\end{array}$ & $\begin{array}{l}\mathrm{CC1CC}=\mathrm{CCCCCCCCCC}= \\
\text { O) } 11\end{array}$ & & Sensitizer & & & & & REACH (2008-2014) & \\
\hline $\begin{array}{l}\text { 3-\{[(5-\{[3- } \\
\text { (dodecanoyloxy)-2,2- } \\
\text { dimethylpropylidene]a } \\
\text { mino\}-1,3,3- } \\
\text { trimethylcyclohexyl)me } \\
\text { thyl]imino\}-2,2- } \\
\text { dimethylpropyl } \\
\text { dodecanoate }\end{array}$ & $\begin{array}{l}\mathrm{CCCCCCCCCCCCC}=\mathrm{O}) \mathrm{OC} \\
\mathrm{C}(\mathrm{C})(\mathrm{C}) \mathrm{C}=\mathrm{NCC} 1(\mathrm{C}) \mathrm{CC}(\mathrm{C} \\
\mathrm{C}(\mathrm{C})(\mathrm{C}) \mathrm{C} 1) \mathrm{N}=\mathrm{CC}(\mathrm{C})(\mathrm{C}) \mathrm{C} \\
\mathrm{OC}(=\mathrm{O}) \mathrm{CCCCCCCCCCC}\end{array}$ & & Sensitizer & & & & & REACH (2008-2014) & \\
\hline $\begin{array}{l}\text { 3-\{[2-(2- } \\
\text { hydroxyethoxy)ethyl]im } \\
\text { ino\}-2,2-dimethylpropyl } \\
\text { dodecanoate }\end{array}$ & $\begin{array}{l}\mathrm{CCCCCCCCCCCC}(=\mathrm{O}) \mathrm{OC} \\
\mathrm{C}(\mathrm{C})(\mathrm{C}) \mathrm{C}=\mathrm{NCCOCCO}\end{array}$ & & Sensitizer & & & & & REACH (2008-2014) & \\
\hline 3H-1-benzofuran-2-one & $\mathrm{O}=\mathrm{C} 1 \mathrm{Cc} 2 \operatorname{ccccc} 2 \mathrm{O} 1$ & & Sensitizer & & & & & REACH (2008-2014) & \\
\hline 4'-Hydroxychalcone & $\begin{array}{l}\text { Oc1 } 1 \operatorname{ccc}(\operatorname{cc} 1) C(=0) C=C c \\
1 \operatorname{ccccc} 1\end{array}$ & & Sensitizer & & & & & ICCVAM (2013) & \\
\hline $\begin{array}{l}\text { 4,4,4-Trifluoro-1- } \\
\text { phenylbutane-1,3- } \\
\text { dione }\end{array}$ & $\begin{array}{l}\mathrm{OC}(=\mathrm{CC}(=0) \operatorname{c} 1 \operatorname{ccccc} 1) \mathrm{C} \\
(\mathrm{F})(\mathrm{F}) \mathrm{F}\end{array}$ & & Sensitizer & & & & & ICCVAM (2013) & \\
\hline 4,4-Dibromobenzil & $\begin{array}{l}\operatorname{Brc1} 1 \mathrm{ccc}(\operatorname{cc} 1) \mathrm{C}(=0) \mathrm{C}(=0 \\
\operatorname{col} \operatorname{ccc}(\mathrm{Br}) \operatorname{cc} 1\end{array}$ & & Sensitizer & & & & & ICCVAM (2013) & \\
\hline $\begin{array}{l}\text { 4,7,7-trimethyl-8- } \\
\text { oxabicyclo[2.2.2]octane }\end{array}$ & ${ }_{2}^{\mathrm{CC} 12 \mathrm{CCC}(\mathrm{CC} 1) \mathrm{C}(\mathrm{C})(\mathrm{C}) \mathrm{O}}$ & & Sensitizer & & & & & REACH (2008-2014) & \\
\hline $\begin{array}{l}\text { 4-((2- } \\
\text { hydroxyethyl)amino)-3- } \\
\text { nitrophenol }\end{array}$ & $\begin{array}{l}\operatorname{OCCNc} 1 \operatorname{ccc}(\mathrm{O}) \operatorname{cc} 1 \mathrm{~N}(=\mathrm{O} \\
)=0\end{array}$ & & Sensitizer & & & & & ICCVAM (2013) & \\
\hline $\begin{array}{l}\text { 4-(2-methylbutan-2- } \\
\text { yl)phenol }\end{array}$ & $\operatorname{CCC}(\mathrm{C})(\mathrm{C}) \operatorname{c1ccc}(\mathrm{O}) \mathrm{cc} 1$ & & Sensitizer & & & & & REACH (2008-2014) & \\
\hline $\begin{array}{l}\text { 4-(2-methylprop-2- } \\
\text { enoyloxy)butyl 2- } \\
\text { methylprop-2-enoate }\end{array}$ & $\begin{array}{l}\mathrm{CC}(=\mathrm{C}) \mathrm{C}(=0) \text { OCCCCOC }( \\
=0) \mathrm{C}(\mathrm{C})=\mathrm{C}\end{array}$ & & Sensitizer & & & & & REACH (2008-2014) & \\
\hline
\end{tabular}




\begin{tabular}{|c|c|c|c|c|c|c|c|c|c|}
\hline Compound name & SMILES & $\begin{array}{l}\text { Human } \\
\text { result }\end{array}$ & $\begin{array}{l}\text { LLNA } \\
\text { result }\end{array}$ & DPRA & KeratinoSens & h-CLAT & $\begin{array}{l}\text { Human } \\
\text { reference }\end{array}$ & LLNA reference & Non-animal reference \\
\hline $\begin{array}{l}\text { 4-(2-phenylpropan-2- } \\
\text { yl)-N-[4-(2- } \\
\text { phenylpropan-2- } \\
\text { yl)phenyl]aniline }\end{array}$ & $\begin{array}{l}\mathrm{CC}(\mathrm{C})(\mathrm{c} 1 \mathrm{ccccc} 1) \mathrm{c} 1 \mathrm{ccc}( \\
\mathrm{Nc2} \operatorname{ccc}(\mathrm{cc} 2) \mathrm{C}(\mathrm{C})(\mathrm{C}) \mathrm{c} 2 \mathrm{cc} \\
\mathrm{ccc} 2) \mathrm{cc} 1\end{array}$ & & Sensitizer & & & & & REACH (2008-2014) & \\
\hline $\begin{array}{l}\text { 4-(4- } \\
\text { hydroxyphenyl)phenol }\end{array}$ & $\begin{array}{l}\text { Oc1ccc(cc1)- } \\
\operatorname{c1ccc}(0) \operatorname{cc} 1\end{array}$ & & Sensitizer & & & & & REACH (2008-2014) & \\
\hline $\begin{array}{l}\text { 4-(bromomethyl)- } \\
\text { benzoic acid ethyl ester }\end{array}$ & $\begin{array}{l}\mathrm{CCOC}(=0) \mathrm{c} 1 \mathrm{ccc}(\mathrm{CBr}) \mathrm{cc} \\
1\end{array}$ & & Sensitizer & & & & & ICCVAM (2013) & \\
\hline $\begin{array}{l}\text { 4-(\{N'-[(2- } \\
\text { hydroxyphenyl)methyli } \\
\text { dene]hydrazinecarbony } \\
\text { l\}methyl)-4- } \\
\text { methylmorpholin-4-ium }\end{array}$ & $\begin{array}{l}\mathrm{C}[\mathrm{N}+] 1(\mathrm{CC}(=\mathrm{O}) \mathrm{NN}=\mathrm{Cc} 2 \\
\operatorname{ccccc} 2 \mathrm{O}) \mathrm{CCOCC} 1\end{array}$ & & Sensitizer & & & & & REACH (2008-2014) & \\
\hline 4-Amino-3-nitrophenol & $\operatorname{Nc} 1 \operatorname{ccc}(0) \operatorname{cc} 1 \mathrm{~N}(=0)=0$ & & Sensitizer & & & & & ICCVAM (2013) & \\
\hline $\begin{array}{l}\text { 4-Amino-3-nitrophenyl } \\
\text { thiocyanate }\end{array}$ & $\begin{array}{l}\operatorname{Nc1} 1 \operatorname{ccc}(\mathrm{SC} \# \mathrm{~N}) \operatorname{cc} 1 \mathrm{~N}(=\mathrm{O}) \\
=0\end{array}$ & & Sensitizer & & & & & ICCVAM (2013) & \\
\hline $\begin{array}{l}\text { 4-Bromo-1- } \\
\text { phthalimidopentane }\end{array}$ & $\begin{array}{l}\mathrm{CC}(\mathrm{Br}) \mathrm{CCCN} 1 \mathrm{C}(=0) \mathrm{c} 2 \mathrm{cc} \\
\mathrm{ccc} 2 \mathrm{C} 1=0\end{array}$ & & Sensitizer & & & & & ICCVAM (2013) & \\
\hline $\begin{array}{l}\text { 4-Chloro-6- } \\
\text { iodoquinazoline }\end{array}$ & Clc1ncnc2ccc(I)cc12 & & Sensitizer & & & & & ICCVAM (2013) & \\
\hline 4-Heptylphenol & cCCCCCCc1ccc(0)cc1 & & Sensitizer & & & & & REACH (2008-2014) & \\
\hline $\begin{array}{l}\text { 4-lodo-1-phthalimido- } \\
\text { pentane }\end{array}$ & $\begin{array}{l}\mathrm{CC}(\mathrm{I}) \mathrm{CCCN} 1 \mathrm{C}(=0) \mathrm{c} 2 \mathrm{ccc} \\
\mathrm{cc} 2 \mathrm{C} 1=0\end{array}$ & & Sensitizer & & & & & ICCVAM (2013) & \\
\hline $\begin{array}{l}\text { 4- } \\
\text { Methylbenzenesulfonyl } \\
\text { chloride }\end{array}$ & $\begin{array}{l}\operatorname{Cc1} \operatorname{ccc}(\operatorname{cc} 1) S(\mathrm{Cl})(=0)= \\
0\end{array}$ & & Sensitizer & & & & & REACH (2008-2014) & \\
\hline $\begin{array}{l}\text { 4- } \\
\text { Morpholinecarboxaldeh } \\
\text { yde }\end{array}$ & $\mathrm{O}=\mathrm{CN} 1 \mathrm{CCOCC} 1$ & & Sensitizer & & & & & REACH (2008-2014) & \\
\hline $\begin{array}{l}\text { 4-Nitrobenzene-1,2- } \\
\text { diamine }\end{array}$ & $\mathrm{Nc} 1 \operatorname{ccc}(\operatorname{cc} 1 \mathrm{~N}) \mathrm{N}(=\mathrm{O})=\mathrm{O}$ & & Sensitizer & & & & & ICCVAM (2013) & \\
\hline 4-Phenylphenol & Oc1ccc(cc1)-c1ccccc1 & & Sensitizer & & & & & REACH (2008-2014) & \\
\hline $\begin{array}{l}\text { 4-Sulfophenyl-6-((1- } \\
\text { oxononyl)amino)hexan } \\
\text { oate } \\
\text { (Nonanoylamidocaproyl } \\
\text { acidoxybenzenesulfona } \\
\text { te) }\end{array}$ & $\begin{array}{l}\operatorname{CCCCCCCCC}(=0) \operatorname{NCCC}( \\
\operatorname{cCC}(0)=0) \operatorname{c} 1 \operatorname{ccccc} 1 \mathrm{~S}(0 \\
)(=0)=0\end{array}$ & & Sensitizer & & & & & ICCVAM (2013) & \\
\hline
\end{tabular}




\begin{tabular}{|c|c|c|c|c|c|c|c|c|c|}
\hline Compound name & SMILES & $\begin{array}{l}\text { Human } \\
\text { result }\end{array}$ & $\begin{array}{l}\text { LLNA } \\
\text { result }\end{array}$ & DPRA & KeratinoSens & h-CLAT & $\begin{array}{l}\text { Human } \\
\text { reference }\end{array}$ & LLNA reference & Non-animal reference \\
\hline $\begin{array}{l}\text { 4-[(2,4-dichloro-5- } \\
\text { methoxyphenyl)amino]- } \\
\text { 6-methoxy-7-[3-(4- } \\
\text { methylpiperazin-1- } \\
\text { yl)propoxy]quinoline-3- } \\
\text { carbonitrile }\end{array}$ & $\begin{array}{l}\operatorname{COc1cc}(\operatorname{Nc2} 2(\operatorname{cnc} 3 c c(O \\
\operatorname{cCCN} 4 \mathrm{CCN}(\mathrm{C}) \mathrm{CC} 4) \mathrm{c}(\mathrm{O} \\
\mathrm{C}) \mathrm{cc} 23) \mathrm{CHN}) \mathrm{c}(\mathrm{Cl}) \mathrm{cc} 1 \mathrm{Cl}\end{array}$ & & Sensitizer & & & & & REACH (2008-2014) & \\
\hline $\begin{array}{l}\text { 4-[1,3-bis(5-tert-butyl- } \\
\text { 4-hydroxy-2- } \\
\text { methylphenyl)butyl]-2- } \\
\text { tert-butyl-5- } \\
\text { methylphenol }\end{array}$ & $\begin{array}{l}\mathrm{CC}(\mathrm{CC}(\mathrm{c} 1 \mathrm{cc}(\mathrm{c}(\mathrm{O}) \mathrm{cc} 1 \mathrm{C}) \mathrm{C} \\
(\mathrm{C})(\mathrm{C}) \mathrm{C}) \mathrm{c} 1 \mathrm{cc}(\mathrm{c}(\mathrm{O}) \mathrm{cc1C}) \\
\mathrm{C}(\mathrm{C})(\mathrm{C}) \mathrm{C}) \mathrm{c} 1 \mathrm{cc}(\mathrm{c}(\mathrm{O}) \mathrm{cc} 1 \mathrm{C} \\
) \mathrm{C}(\mathrm{C})(\mathrm{C}) \mathrm{C}\end{array}$ & & Sensitizer & & & & & REACH (2008-2014) & \\
\hline $\begin{array}{l}\text { 4-[2-ethyl-1-(4- } \\
\text { hydroxyphenyl)hexyl]p } \\
\text { henol }\end{array}$ & $\begin{array}{l}\operatorname{ccCCC}(\operatorname{CC}) \mathrm{C}(\mathrm{c} 1 \mathrm{ccc}(\mathrm{O}) \mathrm{c} \\
\mathrm{c} 1) \operatorname{c1} \mathrm{ccc}(\mathrm{O}) \mathrm{cc} 1\end{array}$ & & Sensitizer & & & & & REACH (2008-2014) & \\
\hline $\begin{array}{l}\text { 4-[[4-(bis(oxiran-2- } \\
\text { ylmethyl)amino)phenyl] } \\
\text { methyl]-N,N-bis(oxiran- } \\
\text { 2-ylmethyl)aniline }\end{array}$ & $\begin{array}{l}\mathrm{C}(\mathrm{C} 1 \mathrm{CO} 1) \mathrm{N}(\mathrm{CC} 1 \mathrm{CO} 1) \mathrm{c} 1 \\
\mathrm{ccc}(\mathrm{Cc} 2 \operatorname{ccc}(\mathrm{cc} 2) \mathrm{N}(\mathrm{CC} 2 \mathrm{C} \\
\mathrm{O} 2) \mathrm{CC} 2 \mathrm{CO} 2) \operatorname{cc} 1\end{array}$ & & Sensitizer & & & & & REACH (2008-2014) & \\
\hline $\begin{array}{l}\text { 4-bromo-2-chloro-1- } \\
\text { (ethoxy- } \\
\text { propylsulfanylphosphor } \\
\text { yl)oxybenzene }\end{array}$ & $\begin{array}{l}\mathrm{CCCSP}(=0)(\mathrm{OCC}) \mathrm{Oc} 1 \mathrm{cc} \\
\mathrm{c}(\mathrm{Br}) \mathrm{cc} 1 \mathrm{Cl}\end{array}$ & & Sensitizer & & & & & REACH (2008-2014) & \\
\hline $\begin{array}{l}\text { 4-methoxyphenyl 4-[4- } \\
\text { (oxiran-2- } \\
\text { yl)butoxy]benzoate }\end{array}$ & $\begin{array}{l}\operatorname{cOc} 1 \mathrm{ccc}(\mathrm{OC}(=\mathrm{O}) \mathrm{c} 2 \mathrm{ccc}( \\
\mathrm{O}(\mathrm{c} C \mathrm{C} 3 \mathrm{CO} 3) \mathrm{cc} 2) \mathrm{cc} 1\end{array}$ & & Sensitizer & & & & & REACH (2008-2014) & \\
\hline $\begin{array}{l}\text { 4-naphthalen-2-yl-1,3- } \\
\text { thiazol-2-amine }\end{array}$ & $\begin{array}{l}\text { Nc1nc(cs1)- } \\
\mathrm{c} 1 \mathrm{ccc} 2 \operatorname{ccccc} 2 \mathrm{c} 1\end{array}$ & & Sensitizer & & & & & REACH (2008-2014) & \\
\hline $\begin{array}{l}\text { 5,5-Dimethyl-3- } \\
\text { methylenedihydro- } \\
\text { 2(3H)-furanone }\end{array}$ & $\mathrm{CC} 1(\mathrm{C}) \mathrm{CC}(=\mathrm{C}) \mathrm{C}(=\mathrm{O}) \mathrm{O} 1$ & & Sensitizer & & & & & ICCVAM (2013) & \\
\hline $\begin{array}{l}\text { 5-Chloro-2,6- } \\
\text { dimethoxy-4-methyl-8- } \\
\text { nitroquinoline }\end{array}$ & $\begin{array}{l}\operatorname{coc} 1 c c(C) c 2 c(C l) c(O C) \\
\operatorname{cc}(c 2 n 1) N(=0)=0\end{array}$ & & Sensitizer & & & & & ICCVAM (2013) & \\
\hline $\begin{array}{l}\text { 5-Chloro-2-hydroxy-3- } \\
\text { nitro-[1,1-biphenyl]-3- } \\
\text { carboxylic acid }\end{array}$ & $\begin{array}{l}\mathrm{OC}(=0) \mathrm{c} 1 \mathrm{cccc}(\mathrm{c} 1)- \\
\mathrm{c} 1 \mathrm{cc}(\mathrm{Cl}) \mathrm{cc}(\mathrm{c} 1 \mathrm{O}) \mathrm{N}(=\mathrm{O})= \\
\mathrm{O}\end{array}$ & & Sensitizer & & & & & ICCVAM (2013) & \\
\hline $\begin{array}{l}\text { 5-Chloro-6-methoxy-4- } \\
\text { methyl-8-nitro- } \\
\text { 2(1H)quinolinone }\end{array}$ & $\begin{array}{l}\operatorname{COc} 1 c c(c 2 N C(=0) C=C( \\
C) \operatorname{coc} 1 C l) N(=0)=O\end{array}$ & & Sensitizer & & & & & ICCVAM (2013) & \\
\hline $\begin{array}{l}\text { 5-Ethyl-2- } \\
\text { methylpyridine }\end{array}$ & $\operatorname{CCc1ccc}(\mathrm{C}) \mathrm{nc1}$ & & Sensitizer & & & & & REACH (2008-2014) & \\
\hline $\begin{array}{l}\text { 5-Methoxy-6- } \\
\text { (trifluoromethyl)-2,3- } \\
\text { dihydro-1H-indole }\end{array}$ & $\begin{array}{l}\operatorname{COc} 1 c c 2 C C N c 2 c c 1 C(F)( \\
F) F\end{array}$ & & Sensitizer & & & & & ICCVAM (2013) & \\
\hline
\end{tabular}




\begin{tabular}{|c|c|c|c|c|c|c|c|c|c|}
\hline Compound name & SMILES & $\begin{array}{l}\text { Human } \\
\text { result }\end{array}$ & $\begin{array}{l}\text { LLNA } \\
\text { result }\end{array}$ & DPRA & KeratinoSens & h-CLAT & $\begin{array}{l}\text { Human } \\
\text { reference }\end{array}$ & LLNA reference & Non-animal reference \\
\hline 5-Methyleugenol & $\operatorname{cOc} 1 \mathrm{cc}(\mathrm{CC}=\mathrm{C}) \mathrm{c}(\mathrm{C}) \mathrm{cc} 10$ & & Sensitizer & & & & & ICCVAM (2013) & \\
\hline $\begin{array}{l}\text { 6-(Diethylamino)-1- } \\
\text { hexanol }\end{array}$ & $\mathrm{CCN}(\mathrm{CC}) \mathrm{CCCCCCO}$ & & Sensitizer & & & & & ICCVAM (2013) & \\
\hline 6-Chlorocoumarin & $\begin{array}{l}\text { Clc1ccc2OC(=O)C=Cc2c } \\
1\end{array}$ & & Sensitizer & & & & & ICCVAM (2013) & \\
\hline $\begin{array}{l}\text { 6-Diethylaminohexyl } \\
\text { bromide hydrobromide }\end{array}$ & $\mathrm{CCN}(\mathrm{CC}) \mathrm{CCCCCCBr}$ & & Sensitizer & & & & & ICCVAM (2013) & \\
\hline 6-Methyleugenol & $\operatorname{cOc} 1 \mathrm{cc}(\mathrm{CC}=\mathrm{C}) \mathrm{cc}(\mathrm{C}) \mathrm{c} 10$ & & Sensitizer & & & & & ICCVAM (2013) & \\
\hline 6-Methylisoeugenol & $\operatorname{cOc} 1 \mathrm{cc}(\mathrm{C}=\mathrm{CC}) \mathrm{cc}(\mathrm{C}) \mathrm{c} 10$ & & Sensitizer & & & & & ICCVAM (2013) & \\
\hline $\begin{array}{l}\text { 6-[(2-Methyl-3- } \\
\text { pyridinyl)oxy]-3- } \\
\text { pyridinamine }\end{array}$ & $\begin{array}{l}\text { Cc1ncccc10c1ccc(N)cn } \\
1\end{array}$ & & Sensitizer & & & & & ICCVAM (2013) & \\
\hline $\begin{array}{l}\text { 7,7-dimethyl-4- } \\
\text { methylidenebicyclo[3.1. } \\
\text { 1]heptane }\end{array}$ & $\mathrm{CC} 1(\mathrm{C}) \mathrm{C} 2 \mathrm{CC} 1 \mathrm{C}(=\mathrm{C}) \mathrm{CC} 2$ & & Sensitizer & & & & & REACH (2008-2014) & \\
\hline 7-Bromotetradecane & $\mathrm{CCCCCCCC}(\mathrm{Br}) \mathrm{CCCCCC}$ & & Sensitizer & & & & & ICCVAM (2013) & \\
\hline $\begin{array}{l}\text { 7-[(4z)-3- } \\
\text { (aminomethyl)-4- } \\
\text { (methoxyimino)-1- } \\
\text { pyrrolidinyl]-1- } \\
\text { cyclopropyl-6-fluoro- } \\
\text { 1,4-dihydro-4-oxo-1,8- } \\
\text { naphthyridine-3- } \\
\text { carboxylic acid, } \\
\text { monomethane- } \\
\text { sulfonate } \\
\end{array}$ & $\begin{array}{l}\mathrm{CON}=\mathrm{C} 1 \mathrm{CN}(\mathrm{CC} 1 \mathrm{CN}) \mathrm{c} 1 \mathrm{n} \\
\mathrm{c} 2 \mathrm{~N}(\mathrm{C}=\mathrm{C}(\mathrm{C}(\mathrm{O})=\mathrm{O}) \mathrm{C}(=\mathrm{O}) \\
\mathrm{c2cc1F}) \mathrm{C1CC1}\end{array}$ & & Sensitizer & & & & & ICCVAM (2013) & \\
\hline $\begin{array}{l}\text { 8-Chloro-3-Pentyl-3,7- } \\
\text { dihydro-1H-purine-2,6- } \\
\text { dione }\end{array}$ & $\begin{array}{l}\text { CCCCCN1c2nc(Cl)[nH]c } \\
2 \mathrm{C}(=\mathrm{O}) \mathrm{NC} 1=\mathrm{O}\end{array}$ & & Sensitizer & & & & & ICCVAM (2013) & \\
\hline $\begin{array}{l}\text { 8-Methyl-8- } \\
\text { azabicyclo[3.2.1]octan- } \\
\text { 3-ol }\end{array}$ & $\mathrm{CN} 1 \mathrm{C} 2 \mathrm{CCC} 1 \mathrm{CC}(\mathrm{O}) \mathrm{C2}$ & & Sensitizer & & & & & ICCVAM (2013) & \\
\hline $\begin{array}{l}\text { 8-methylnonyl prop-2- } \\
\text { enoate }\end{array}$ & $\begin{array}{l}\mathrm{CC}(\mathrm{C}) \operatorname{cccccccoc}(=0) \\
\mathrm{C}=\mathrm{C}\end{array}$ & & Sensitizer & & & & & REACH (2008-2014) & \\
\hline Acetyl cedrene & $\begin{array}{l}\mathrm{CC1CCC2C}(C)(C) \mathrm{C} 3 \mathrm{CC} 1 \\
2 \mathrm{CC}(\mathrm{C}(\mathrm{C})=\mathrm{O})=\mathrm{C} 3 \mathrm{C}\end{array}$ & & Sensitizer & & & & & $\begin{array}{l}\text { ICCVAM }(2013)+ \\
\operatorname{REACH}(2008-2014)\end{array}$ & \\
\hline Alachlor & $\begin{array}{l}\mathrm{CCc} 1 \mathrm{cccc}(\mathrm{CC}) \mathrm{c} 1 \mathrm{~N}(\mathrm{COC}) \\
\mathrm{C}(=0) \mathrm{CCl}\end{array}$ & & Sensitizer & & & & & ICCVAM (2013) & \\
\hline Atranol & $\operatorname{Cc} 1 \mathrm{cc}(\mathrm{O}) \mathrm{c}(\mathrm{C}=\mathrm{O}) \mathrm{c}(\mathrm{O}) \mathrm{c1}$ & & Sensitizer & & & & & ICCVAM (2013) & \\
\hline
\end{tabular}




\begin{tabular}{|c|c|c|c|c|c|c|c|c|c|}
\hline Compound name & SMILES & $\begin{array}{l}\text { Human } \\
\text { result }\end{array}$ & $\begin{array}{l}\text { LLNA } \\
\text { result }\end{array}$ & DPRA & KeratinoSens & h-CLAT & $\begin{array}{l}\text { Human } \\
\text { reference }\end{array}$ & LLNA reference & Non-animal reference \\
\hline Azithromycin & $\begin{array}{l}\mathrm{CCC1OC}(=0) C(C) C(O C 2 \\
\mathrm{CC}(\mathrm{C})(\mathrm{OC}) \mathrm{C}(\mathrm{O}) \mathrm{C}(\mathrm{C}) \mathrm{O} 2) \\
\mathrm{C}(\mathrm{C}) \mathrm{C}(\mathrm{OC} 2 \mathrm{OC}(\mathrm{C}) \mathrm{CC}(\mathrm{C} 2 \\
\mathrm{O}) \mathrm{N}(\mathrm{C}) \mathrm{C}) \mathrm{C}(\mathrm{C})(\mathrm{O}) \mathrm{CC}(\mathrm{C}) \mathrm{C} \\
\mathrm{N}(\mathrm{C}) \mathrm{C}(\mathrm{C}) \mathrm{C}(\mathrm{O}) \mathrm{C} 1(\mathrm{C}) \mathrm{O}\end{array}$ & & Sensitizer & & & & & ICCVAM (2013) & \\
\hline Benzene, 2-bromoethyl & BrCCc1ccccc1 & & Sensitizer & & & & & ICCVAM (2013) & \\
\hline $\begin{array}{l}\text { Benzyl N-\{1-[(2- } \\
\text { aminoethyl)amino]-3- } \\
\text { (4- } \\
\text { ethoxyphenyl)propan- } \\
\text { 2-yl\}carbamate }\end{array}$ & $\begin{array}{l}\text { CCOc1ccc(CC(CNCCN) } \\
\text { NC(=0)OCc2 } \operatorname{ccccc} 2) \operatorname{cc} 1\end{array}$ & & Sensitizer & & & & & REACH (2008-2014) & \\
\hline $\begin{array}{l}\text { Bis[4-(2-methyl-2- } \\
\text { propanyl)phenyl]iodoni } \\
\text { um } \\
\text { hexafluorophosphate }\end{array}$ & $\begin{array}{l}\mathrm{CC}(\mathrm{C})(\mathrm{C})[\mathrm{C}- \\
] 1 \mathrm{C}=\mathrm{CC}(\mathrm{C}=\mathrm{C} 1)=[1++] \mathrm{c} 1 \mathrm{c} \\
\operatorname{cc}(\mathrm{cc} 1) \mathrm{C}(\mathrm{C})(\mathrm{C}) \mathrm{C}\end{array}$ & & Sensitizer & & & & & REACH (2008-2014) & \\
\hline $\begin{array}{l}\text { Butanoic acid, 3- } \\
\text { methyl, 2-phenylethyl } \\
\text { ester }\end{array}$ & $\begin{array}{l}\mathrm{CC}(\mathrm{C}) \mathrm{CC}(=0) 0 \mathrm{OCC} 1 \mathrm{cccc} \\
\mathrm{c1}\end{array}$ & & Sensitizer & & & & & ICCVAM (2013) & \\
\hline C.I. Reactive Red 231 & 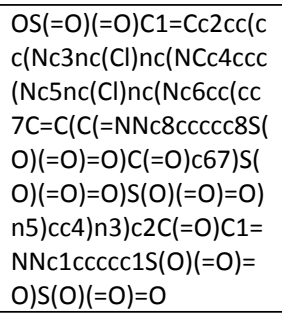 & & Sensitizer & & & & & ICCVAM (2013) & \\
\hline C.I. Reactive Yellow 174 & $\begin{array}{l}\mathrm{NC}(=0) \operatorname{Nc} 1 \mathrm{cc}(\mathrm{Nc} 2 \mathrm{nc}(\mathrm{F}) \\
\mathrm{nc}(\mathrm{NCCOCCS}(=0)(=0) \mathrm{C} \\
=\mathrm{C}) \mathrm{n} 2) \operatorname{ccc} 1 \mathrm{~N}=\mathrm{Nc1} 1 \mathrm{cc} 2 \mathrm{c}( \\
\mathrm{cc}(\mathrm{cc} 2 \mathrm{cc} 1 \mathrm{~S}(\mathrm{O})(=0)=0) \mathrm{S} \\
(0)(=0)=0) \mathrm{S}(\mathrm{O})(=0)=0\end{array}$ & & Sensitizer & & & & & ICCVAM (2013) & \\
\hline C11 Azlactone & $\begin{array}{l}\text { CCCCCCCCCCCC1 } 1=N C( \\
\text { C) }(C) C(=0) O 1\end{array}$ & & Sensitizer & & & & & ICCVAM (2013) & \\
\hline C15 Azlactone & $\begin{array}{l}\text { CCCCCCCCCCCCCCCC1 } \\
=\mathrm{NC}(\mathrm{C})(\mathrm{C}) \mathrm{C}(=0) \mathrm{O} 1\end{array}$ & & Sensitizer & & & & & ICCVAM (2013) & \\
\hline C17 Azlactone & $\begin{array}{l}\mathrm{CCCCCCCCCCCCCCCCC} \\
\mathrm{C} 1=\mathrm{NC}(\mathrm{C})(\mathrm{C}) \mathrm{C}(=\mathrm{O}) \mathrm{O} 1\end{array}$ & & Sensitizer & & & & & ICCVAM (2013) & \\
\hline C19 Azlactone & $\begin{array}{l}\text { CCCCCCCCCCCCCCCCC } \\
\mathrm{CCC} 1=N C(C)(C) C(=0) 0 \\
1\end{array}$ & & Sensitizer & & & & & ICCVAM (2013) & \\
\hline C4 Azlactone & $\begin{array}{l}\text { CCCCC1=NC(C)(C)C }(=0 \\
101\end{array}$ & & Sensitizer & & & & & ICCVAM (2013) & \\
\hline
\end{tabular}




\begin{tabular}{|c|c|c|c|c|c|c|c|c|c|}
\hline Compound name & SMILES & $\begin{array}{l}\text { Human } \\
\text { result }\end{array}$ & $\begin{array}{l}\text { LLNA } \\
\text { result }\end{array}$ & DPRA & KeratinoSens & h-CLAT & $\begin{array}{l}\text { Human } \\
\text { reference }\end{array}$ & LLNA reference & Non-animal reference \\
\hline C6 Azlactone & $\begin{array}{l}\mathrm{CCCCCCC1}=\mathrm{NC}(\mathrm{C})(\mathrm{C}) \mathrm{C}( \\
=0) 01\end{array}$ & & Sensitizer & & & & & ICCVAM (2013) & \\
\hline C9 Azlactone & $\begin{array}{l}\text { CCCCCCCCCC1 } 1=N C(C)( \\
\text { C) }(=0) 01\end{array}$ & & Sensitizer & & & & & ICCVAM (2013) & \\
\hline Camphorquinone & $\begin{array}{l}\mathrm{CC} 1(\mathrm{C}) \mathrm{C} 2 \mathrm{CCC} 1(\mathrm{C}) \mathrm{C}(=0) \\
\mathrm{C} 2=0\end{array}$ & & Sensitizer & & & & & ICCVAM (2013) & \\
\hline Chloroantrol & $\begin{array}{l}\mathrm{Cc} 1 \mathrm{cc}(\mathrm{O}) \mathrm{c}(\mathrm{C}=\mathrm{O}) \mathrm{c}(\mathrm{O}) \mathrm{c1} \\
\mathrm{Cl}\end{array}$ & & Sensitizer & & & & & ICCVAM (2013) & \\
\hline Chloromethylbenzene & ClCc1ccccc1 & & Sensitizer & & & & & REACH (2008-2014) & \\
\hline Chlorosalicylanilide & $\begin{array}{l}\text { Oc1ccc }(\mathrm{Cl}) \operatorname{cc} 1 \mathrm{C}(=\mathrm{O}) \mathrm{Nc} \\
1 \mathrm{ccccc} 1\end{array}$ & & Sensitizer & & & & & ICCVAM (2013) & \\
\hline $\begin{array}{l}\text { Cyclohex-3-ene-1- } \\
\text { carboxylic acid }\end{array}$ & $\mathrm{OC}(=\mathrm{O}) \mathrm{C} 1 \mathrm{CCC}=\mathrm{CC} 1$ & & Sensitizer & & & & & ICCVAM (2013) & \\
\hline $\begin{array}{l}\text { Diazanium sulfonatooxy } \\
\text { sulfate }\end{array}$ & $\begin{array}{l}\operatorname{OS}(=0)(=0) \operatorname{OOS}(0)(=0 \\
)=0\end{array}$ & & Sensitizer & & & & & REACH (2008-2014) & \\
\hline Dibenzodioxocin & $\begin{array}{l}\mathrm{c} 1 \mathrm{ccc} 2 \mathrm{c}(\mathrm{c} 1) \mathrm{ccooc} 1 \mathrm{cccc} \\
\mathrm{c} 21\end{array}$ & & Sensitizer & & & & & ICCVAM (2013) & \\
\hline $\begin{array}{l}\text { Dicyclohexyl benzene- } \\
\text { 1,2-dicarboxylate }\end{array}$ & $\begin{array}{l}\mathrm{O}=\mathrm{C}(\mathrm{OC} 1 \mathrm{CCCCC} 1) \mathrm{c} 1 \mathrm{ccc} \\
\mathrm{cc} 1 \mathrm{C}(=\mathrm{O}) \mathrm{OC} 1 \mathrm{CCCCC} 1\end{array}$ & & Sensitizer & & & & & REACH (2008-2014) & \\
\hline $\begin{array}{l}\text { Dicyclohexylcarbodiimi } \\
\text { de }\end{array}$ & $\begin{array}{l}\mathrm{C} 1 \mathrm{CCC}(\mathrm{CC} 1) \mathrm{N}=\mathrm{C}=\mathrm{NC} 1 \mathrm{C} \\
\mathrm{CCCC} 1\end{array}$ & & Sensitizer & & & & & ICCVAM (2013) & \\
\hline Diethylamine & CCNCC & & Sensitizer & & & & & ICCVAM (2013) & \\
\hline Dimethyl sulfate & $\cos (=0)(=0) O C$ & & Sensitizer & & & & & ICCVAM (2013) & \\
\hline $\begin{array}{l}\text { Dimethyldithiocarbama } \\
\text { te }\end{array}$ & $\mathrm{CN}(\mathrm{C}) \mathrm{C}(\mathrm{S})=\mathrm{S}$ & & Sensitizer & & & & & ICCVAM (2013) & \\
\hline Dinocap & $\begin{array}{l}\mathrm{cCCCCCC}(\mathrm{C}) \mathrm{c} 1 \mathrm{cc}(\mathrm{ccc}(\mathrm{c} 1 \\
\mathrm{OC}(=\mathrm{O}) \mathrm{C}=\mathrm{CC}) \mathrm{N}(=\mathrm{O})=0) \\
\mathrm{N}(=\mathrm{O})=\mathrm{O}\end{array}$ & & Sensitizer & & & & & ICCVAM (2013) & \\
\hline Dipropylene triamine & NCCCNCCCN & & Sensitizer & & & & & $\begin{array}{l}\text { ICCVAM }(2013)+ \\
\operatorname{REACH}(2008-2014)\end{array}$ & \\
\hline Disperse Blue 106 & $\begin{array}{l}\mathrm{CCN}(\mathrm{CCO}) \mathrm{c} 1 \mathrm{ccc}(\mathrm{N}=\mathrm{Nc} 2 \\
\mathrm{ncc}(\mathrm{s} 2) \mathrm{N}(=\mathrm{O})=\mathrm{O}) \mathrm{c}(\mathrm{C}) \mathrm{c1}\end{array}$ & & Sensitizer & & & & & ICCVAM (2013) & \\
\hline $\begin{array}{l}\text { Dodecyl } \\
\text { methanesulfonate }\end{array}$ & $\begin{array}{l}\operatorname{ccccccccccccos}(\mathrm{C})( \\
=0)=0\end{array}$ & & Sensitizer & & & & & ICCVAM (2013) & \\
\hline $\begin{array}{l}\text { Endo-tropine-3- } \\
\text { mesylate }\end{array}$ & $\begin{array}{l}\text { CN1C2CCC1CC(C2)OS( } \\
C)(=0)=0\end{array}$ & & Sensitizer & & & & & ICCVAM (2013) & \\
\hline $\begin{array}{l}\text { Ethyl (3-endo)-8- } \\
\text { methyl-8- } \\
\text { azabicyclo[3.2.1]- } \\
\text { octane-3-acetate }\end{array}$ & $\begin{array}{l}\mathrm{CCOC}(=0) \mathrm{CC} 1 \mathrm{CC} 2 \mathrm{CCC}( \\
\mathrm{C} 1) \mathrm{N} 2 \mathrm{C}\end{array}$ & & Sensitizer & & & & & ICCVAM (2013) & \\
\hline Ethyl 4-iodobenzoate & $\operatorname{ccOC}(=0) \operatorname{coccc}(I) \operatorname{cc} 1$ & & Sensitizer & & & & & ICCVAM (2013) & \\
\hline
\end{tabular}




\begin{tabular}{|c|c|c|c|c|c|c|c|c|c|}
\hline Compound name & SMILES & $\begin{array}{l}\text { Human } \\
\text { result }\end{array}$ & $\begin{array}{l}\text { LLNA } \\
\text { result }\end{array}$ & DPRA & KeratinoSens & h-CLAT & $\begin{array}{l}\text { Human } \\
\text { reference }\end{array}$ & LLNA reference & Non-animal reference \\
\hline $\begin{array}{l}\text { Ethyl-2- } \\
\text { (Hydroxymethyl)-1,3- } \\
\text { Propanediol Triacrylate }\end{array}$ & $\begin{array}{l}\operatorname{CCC}(\operatorname{COC}(=0) C=C)(\operatorname{COC} \\
(=0) C=C) \operatorname{COC}(=O) C=C\end{array}$ & & Sensitizer & & & & & ICCVAM (2013) & \\
\hline $\begin{array}{l}\text { Formulation } 10 \\
\text { (Dithiopyr) }\end{array}$ & $\begin{array}{l}\mathrm{CSC}(=0) \mathrm{c} 1 \mathrm{c}(\mathrm{nc}(\mathrm{c}(\mathrm{C}(=\mathrm{O}) \\
\mathrm{SC}) \mathrm{c} 1 \mathrm{CC}(\mathrm{C}) \mathrm{C}) \mathrm{C}(\mathrm{F})(\mathrm{F}) \mathrm{F}) \mathrm{C} \\
(\mathrm{F}) \mathrm{F}\end{array}$ & & Sensitizer & & & & & ICCVAM (2013) & \\
\hline $\begin{array}{l}\text { Formulation } 13 \text { (2- } \\
\text { ethylhexyl 2-(2,4- } \\
\text { dichlorophenoxy)acetat } \\
\text { e) }\end{array}$ & $\begin{array}{l}\mathrm{CCCCC}(\mathrm{CC}) \operatorname{COC}(=0) \mathrm{CO} \\
\operatorname{c1ccc}(\mathrm{Cl}) \operatorname{cc} 1 \mathrm{Cl}\end{array}$ & & Sensitizer & & & & & ICCVAM (2013) & \\
\hline $\begin{array}{l}\text { Formulation } 16 \\
\text { (Triclopyr ester) }\end{array}$ & $\begin{array}{l}\mathrm{cCCCOCCOC}(=0) \operatorname{COc} 1 \\
\mathrm{nc}(\mathrm{Cl}) \mathrm{c}(\mathrm{Cl}) \mathrm{cc} 1 \mathrm{Cl}\end{array}$ & & Sensitizer & & & & & ICCVAM (2013) & \\
\hline $\begin{array}{l}\text { Formulation } 24 \\
\text { (Cloquintocet-mexyl) }\end{array}$ & $\begin{array}{l}\mathrm{CCCCCC}(\mathrm{C}) \mathrm{OC}(=0) \mathrm{COc} \\
1 \mathrm{ccc}(\mathrm{Cl}) \mathrm{c} 2 \mathrm{ccc} n \mathrm{cc} 12\end{array}$ & & Sensitizer & & & & & $\begin{array}{l}\text { ICCVAM }(2013)+ \\
\text { REACH }(2008-2014)\end{array}$ & \\
\hline $\begin{array}{l}\text { Formulation } 27 \\
\text { (Fluroxypyr-meptyl) }\end{array}$ & $\begin{array}{l}\mathrm{CCCCCCC}(\mathrm{C}) \mathrm{OC}(=0) \mathrm{CO} \\
\mathrm{c} 1 \mathrm{nc}(\mathrm{F}) \mathrm{c}(\mathrm{Cl}) \mathrm{c}(\mathrm{N}) \mathrm{c} 1 \mathrm{Cl}\end{array}$ & & Sensitizer & & & & & ICCVAM (2013) & \\
\hline $\begin{array}{l}\text { Formulation } 32 \\
\text { (Myclobutanil) }\end{array}$ & $\begin{array}{l}\operatorname{CCCCC}(\mathrm{Cn} 1 \mathrm{cncn} 1)(\mathrm{C \# N} \\
) \operatorname{c1ccc}(\mathrm{Cl}) \mathrm{cc} 1\end{array}$ & & Sensitizer & & & & & ICCVAM (2013) & \\
\hline $\begin{array}{l}\text { Formulation } 38 \text { ( } \mathrm{N}-(3,4- \\
\text { dichlorophenyl)propana } \\
\text { mide) }\end{array}$ & $\begin{array}{l}\mathrm{CCC}(=0) \mathrm{Nc} 1 \mathrm{ccc}(\mathrm{Cl}) \mathrm{c}(\mathrm{Cl}) \\
\mathrm{c1}\end{array}$ & & Sensitizer & & & & & ICCVAM (2013) & \\
\hline $\begin{array}{l}\text { Formulation } 56 \\
\text { (Nitrapyrin) }\end{array}$ & Clc1cccc(n1)C(Cl)(Cl)Cl & & Sensitizer & & & & & ICCVAM (2013) & \\
\hline Glygeryl thioglycollate & $\mathrm{OCC}(\mathrm{O}) \mathrm{COC}(=\mathrm{O}) \mathrm{CS}$ & & Sensitizer & & & & & ICCVAM (2013) & \\
\hline Glyoxylic acid & $\mathrm{OC}(=\mathrm{O}) \mathrm{C}=\mathrm{O}$ & & Sensitizer & & & & & $\begin{array}{l}\text { ICCVAM }(2013)+ \\
\text { REACH }(2008-2014)\end{array}$ & \\
\hline Hexamine & $\begin{array}{l}\mathrm{C} 1 \mathrm{~N} 2 \mathrm{CN} 3 \mathrm{CN} 1 \mathrm{CN}(\mathrm{C} 2) \mathrm{C} \\
3\end{array}$ & & Sensitizer & & & & & REACH (2008-2014) & \\
\hline Hydroxytyrosol & OCCc1ccc(O)c(O)c1 & & Sensitizer & & & & & ICCVAM (2013) & \\
\hline $\begin{array}{l}\text { Isodecyl } \\
\text { diphenylphosphite }\end{array}$ & $\begin{array}{l}\mathrm{CC}(\mathrm{C}) \mathrm{CCCCCCCOP}(\mathrm{Oc} 1 \\
\operatorname{ccccc} 1) \text { Oc1 } 1 \mathrm{ccccc} 1\end{array}$ & & Sensitizer & & & & & REACH (2008-2014) & \\
\hline Isononanoyl chloride & $\mathrm{CC}(\mathrm{C}) \operatorname{cccccC}(\mathrm{Cl})=\mathrm{O}$ & & Sensitizer & & & & & ICCVAM (2013) & \\
\hline Isopropyl isoeugenol & $\begin{array}{l}\mathrm{CC}=\mathrm{Cc} 1 \mathrm{ccc}(\mathrm{O}) \mathrm{c}(\mathrm{OC}(\mathrm{C}) \mathrm{C} \\
\mathrm{c} 1\end{array}$ & & Sensitizer & & & & & ICCVAM (2013) & \\
\hline Linalool aldehyde & $\begin{array}{l}\mathrm{CC}(\mathrm{C}=\mathrm{O})=\mathrm{CCCC}(\mathrm{C})(\mathrm{O}) \mathrm{C} \\
=\mathrm{C}\end{array}$ & & Sensitizer & & & & & ICCVAM (2013) & \\
\hline Linoleic acid & $\begin{array}{l}\mathrm{CCCCCC}=\mathrm{CCC}=\mathrm{CCCCCC} \\
\mathrm{CCC}(\mathrm{O})=\mathrm{O}\end{array}$ & & Sensitizer & & & & & ICCVAM (2013) & \\
\hline Linolenic acid & $\begin{array}{l}\mathrm{CCC}=\mathrm{CCC}=\mathrm{CCC}=\mathrm{CCCCC} \\
\mathrm{CCCC}(\mathrm{O})=\mathrm{O}\end{array}$ & & Sensitizer & & & & & ICCVAM (2013) & \\
\hline $\begin{array}{l}\text { Methyl 3-amino-5,6- } \\
\text { dichloropyrazine-2- } \\
\text { carboxylate }\end{array}$ & $\begin{array}{l}\operatorname{COC}(=0) c 1 n c(C l) c(C l) n \\
\operatorname{c1N}\end{array}$ & & Sensitizer & & & & & REACH (2008-2014) & \\
\hline
\end{tabular}




\begin{tabular}{|c|c|c|c|c|c|c|c|c|c|}
\hline Compound name & SMILES & $\begin{array}{l}\text { Human } \\
\text { result }\end{array}$ & $\begin{array}{l}\text { LLNA } \\
\text { result }\end{array}$ & DPRA & KeratinoSens & h-CLAT & $\begin{array}{l}\text { Human } \\
\text { reference }\end{array}$ & LLNA reference & Non-animal reference \\
\hline $\begin{array}{l}\text { Methyl 4- } \\
\text { (bromomethyl)benzoat } \\
\text { e }\end{array}$ & $\operatorname{COC}(=0) \operatorname{c1ccc}(\mathrm{CBr}) \mathrm{cc} 1$ & & Sensitizer & & & & & ICCVAM (2013) & \\
\hline $\begin{array}{l}\text { Methyl } \\
\text { dodecanesulfonate }\end{array}$ & $\begin{array}{l}\operatorname{cccccccccccccs}(=0)( \\
=0) 0 C\end{array}$ & & Sensitizer & & & & & ICCVAM (2013) & \\
\hline $\begin{array}{l}\text { Methyl } \\
\text { hexadecenesulfonate }\end{array}$ & $\begin{array}{l}\mathrm{CcCcccccccccccc}=\mathrm{C} \\
\mathrm{S}(=\mathrm{O})(=\mathrm{O}) \mathrm{OC}\end{array}$ & & Sensitizer & & & & & ICCVAM (2013) & \\
\hline $\begin{array}{l}\text { N,N'-Bis[2-hydroxy-3- } \\
(2,2,3,3- \\
\text { tetrafluoropropoxy)pro } \\
\text { pyl]-N,N,N',N'- } \\
\text { tetramethyl-1,2- } \\
\text { ethanediaminium } \\
\text { dichloride }\end{array}$ & $\begin{array}{l}\mathrm{C}[\mathrm{N}+](\mathrm{C})(\mathrm{CC}[\mathrm{N}+](\mathrm{C})(\mathrm{C}) \mathrm{C} \\
\mathrm{C}(\mathrm{O}) \operatorname{COCC}(\mathrm{F})(\mathrm{F}) \mathrm{C}(\mathrm{F}) \mathrm{F}) \mathrm{C} \\
\mathrm{C}(\mathrm{O}) \operatorname{COCC}(\mathrm{F})(\mathrm{F}) \mathrm{C}(\mathrm{F}) \mathrm{F}\end{array}$ & & Sensitizer & & & & & REACH (2008-2014) & \\
\hline $\begin{array}{l}\mathrm{N}-(2 \text {-Hydroxyethyl)- } \\
\text { ethylendiamine }\end{array}$ & NCCNCCO & & Sensitizer & & & & & ICCVAM (2013) & \\
\hline $\begin{array}{l}\mathrm{N} \text {-(3,4-Dichlorophenyl)- } \\
\mathrm{N} \text {-(1-methylethyl)- } \\
\text { imidodicarbonimidic } \\
\text { diamide } \\
\text { monohydrochloride }\end{array}$ & $\begin{array}{l}\mathrm{CC}(\mathrm{C}) \mathrm{NC}(=\mathrm{N}) \mathrm{NC}(=\mathrm{N}) \mathrm{Nc} \\
1 \mathrm{ccc}(\mathrm{Cl}) \mathrm{c}(\mathrm{Cl}) \mathrm{c1}\end{array}$ & & Sensitizer & & & & & ICCVAM (2013) & \\
\hline $\begin{array}{l}\mathrm{N}-(3- \\
\text { dimethylaminopropyl)- } \\
\text { 2-methylprop-2- } \\
\text { enamide }\end{array}$ & $\begin{array}{l}\mathrm{CN}(\mathrm{C}) \operatorname{CCCNC}(=\mathrm{O}) \mathrm{C}(\mathrm{C})= \\
\mathrm{C}\end{array}$ & & Sensitizer & & & & & REACH (2008-2014) & \\
\hline $\begin{array}{l}\mathrm{N}-(3- \\
\text { trimethoxysilylpropyl)et } \\
\text { hane-1,2-diamine } \\
\text { hydrochloride }\end{array}$ & $\begin{array}{l}\mathrm{CO}[\mathrm{Si}](\mathrm{CCCNCCN})(\mathrm{OC}) \\
\mathrm{OC}\end{array}$ & & Sensitizer & & & & & REACH (2008-2014) & \\
\hline $\begin{array}{l}\mathrm{N} \text {-(4-Methoxyphenyl)- } \\
\text { 3-oxobutanamide }\end{array}$ & $\begin{array}{l}\operatorname{COc1ccc}(\mathrm{NC}(=0) \mathrm{CC}(\mathrm{C})= \\
0) \operatorname{cc} 1\end{array}$ & & Sensitizer & & & & & ICCVAM (2013) & \\
\hline N-Ethyl-N-nitrosourea & $\mathrm{CCN}(\mathrm{N}=\mathrm{O}) \mathrm{C}(\mathrm{N})=\mathrm{O}$ & & Sensitizer & & & & & ICCVAM (2013) & \\
\hline $\begin{array}{l}\mathrm{N} \text {-Isopropyl-N-phenyl- } \\
\text { 2-(2-phenylamino- } \\
\text { phenylamino)- } \\
\text { acetamide }\end{array}$ & $\begin{array}{l}\mathrm{CC}(\mathrm{C}) \mathrm{N}(\mathrm{C}(=\mathrm{O}) \mathrm{CN} 1 \mathrm{cccc} \\
\mathrm{c} 1 \mathrm{Nc} 1 \mathrm{ccccc} 1) \mathrm{c} 1 \mathrm{ccccc} 1\end{array}$ & & Sensitizer & & & & & ICCVAM (2013) & \\
\hline
\end{tabular}




\begin{tabular}{|c|c|c|c|c|c|c|c|c|c|}
\hline Compound name & SMILES & $\begin{array}{l}\text { Human } \\
\text { result }\end{array}$ & $\begin{array}{l}\text { LLNA } \\
\text { result }\end{array}$ & DPRA & KeratinoSens & h-CLAT & $\begin{array}{l}\text { Human } \\
\text { reference }\end{array}$ & LLNA reference & Non-animal reference \\
\hline $\begin{array}{l}\mathrm{N}-[2- \\
\text { (diethylamino)ethyl]-2- } \\
{[[(4-\text { fluorophenyl)- }} \\
\text { methyl]thio]-4,5,6,7- } \\
\text { tetrahydro-4-oxo-N- } \\
{\left[\left[4^{\prime}-\text {-(trifluoromethyl)- }\right.\right.} \\
{\left[1,1^{\prime} \text {-biphenyl]-4- }\right.} \\
\text { yl]methyl]-1h- } \\
\text { cyclopentapyrim-idine- } \\
\text { 1-acetamide } \\
\text { (Darapladib) }\end{array}$ & $\begin{array}{l}\mathrm{CCN}(\mathrm{CC}) \mathrm{CCN}(\mathrm{Cc} 1 \mathrm{ccc}(\mathrm{cc} \\
\text { 1)- } \\
\mathrm{c1} 1 \mathrm{ccc}(\mathrm{cc} 1) \mathrm{C}(\mathrm{F})(\mathrm{F}) \mathrm{F}) \mathrm{C}(= \\
\mathrm{O}) \mathrm{CN} 1 \mathrm{C} 2=\mathrm{C}(\mathrm{CCC} 2) \mathrm{C}= \\
\mathrm{O}=\mathrm{N}=\mathrm{C} 1 \mathrm{SCc} 1 \mathrm{ccc}(\mathrm{F}) \mathrm{cc} 1\end{array}$ & & Sensitizer & & & & & ICCVAM (2013) & \\
\hline $\begin{array}{l}\mathrm{N}-[3- \\
\text { (triethoxysilyl)propyl]fo } \\
\text { rmamide }\end{array}$ & $\begin{array}{l}\mathrm{CCO}[\mathrm{Si}](\mathrm{CCCNC}=0)(\mathrm{OC} \\
\mathrm{C}) \mathrm{OCC}\end{array}$ & & Sensitizer & & & & & REACH (2008-2014) & \\
\hline $\begin{array}{l}\mathrm{N} \text {-isopropyl-N'-phenyl- } \\
\mathrm{p} \text {-phenylenediamine }\end{array}$ & $\begin{array}{l}\mathrm{CC}(\mathrm{C}) \mathrm{Nc} 1 \mathrm{ccc}(\mathrm{Nc} 2 \mathrm{ccccc} \\
\text { 2) } \mathrm{cc} 1\end{array}$ & & Sensitizer & & & & & ICCVAM (2013) & \\
\hline $\begin{array}{l}\text { N-phenyl-N- } \\
\text { (trichloromethylsulfanyl } \\
\text { )benzenesulfonamide }\end{array}$ & $\begin{array}{l}\mathrm{CIC}(\mathrm{Cl})(\mathrm{Cl}) \mathrm{SN}(\mathrm{c} 1 \mathrm{cccccc} 1) \\
\mathrm{S}(=0)(=0) \mathrm{c} 1 \mathrm{ccccc} 1\end{array}$ & & Sensitizer & & & & & REACH (2008-2014) & \\
\hline $\begin{array}{l}\text { N4-[3-[[4,6-bis[butyl- } \\
(1,2,2,6,6- \\
\text { pentamethylpiperidin- } \\
4 \text {-yl)amino]-1,3,5- } \\
\text { triazin-2- } \\
\text { yl]amino]propyl]-N4-[2- } \\
{[[4,6-\text { bis[butyl- }} \\
(1,2,2,6,6- \\
\text { pentamethylpiperidin- } \\
\text { 4-yl)amino]-1,3,5- } \\
\text { triazin-2-yl]-[3-[[4,6- } \\
\text { bis[butyl-(1,2,2,6,6- } \\
\text { pentamethylpiperidin- } \\
\text { 4-yl)amino]-1,3,5- } \\
\text { triazin-2- } \\
\text { yl]amino]propyl]amino] } \\
\text { ethyl]-N2,N6-dibutyl- } \\
\text { N2,N6-bis(1,2,2,6,6- } \\
\text { pentamethylpiperidin- } \\
\text { 4-yl)-1,3,5-triazine- } \\
\text { 2,4,6-triamine }\end{array}$ & 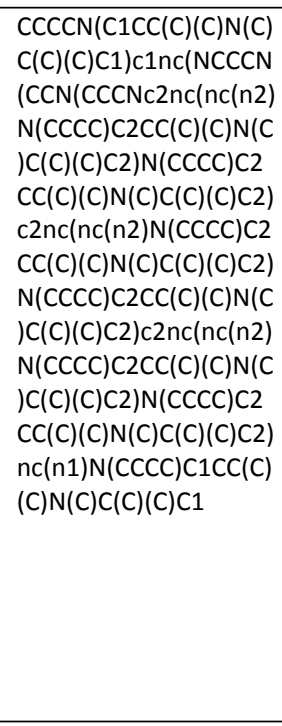 & & Sensitizer & & & & & REACH (2008-2014) & \\
\hline Nonanoyl chloride & $\operatorname{ccccccccc}(\mathrm{Cl})=0$ & & Sensitizer & & & & & ICCVAM (2013) & \\
\hline $\begin{array}{l}\text { Norbornene } \\
\text { fluoroalcohol }\end{array}$ & $\begin{array}{l}\mathrm{OC}(\mathrm{COC} 1 \mathrm{CC} 2 \mathrm{CC} 1 \mathrm{C}=\mathrm{C} 2) \\
(\mathrm{C}(\mathrm{F})(\mathrm{F}) \mathrm{F}) \mathrm{C}(\mathrm{F})(\mathrm{F}) \mathrm{F}\end{array}$ & & Sensitizer & & & & & ICCVAM (2013) & \\
\hline Oct-1-yn-3-ol & $\operatorname{CCCCCC}(0) \mathrm{C \# C}$ & & Sensitizer & & & & & ICCVAM (2013) & \\
\hline
\end{tabular}




\begin{tabular}{|c|c|c|c|c|c|c|c|c|c|}
\hline Compound name & SMILES & $\begin{array}{l}\text { Human } \\
\text { result }\end{array}$ & $\begin{array}{l}\text { LLNA } \\
\text { result }\end{array}$ & DPRA & KeratinoSens & h-CLAT & $\begin{array}{l}\text { Human } \\
\text { reference }\end{array}$ & LLNA reference & Non-animal reference \\
\hline Oleic acid & $\begin{array}{l}\mathrm{CCCCCCCCC}=\mathrm{CCCCCCC} \\
\mathrm{CC}(\mathrm{O})=0\end{array}$ & & Sensitizer & & & & & ICCVAM (2013) & \\
\hline $\begin{array}{l}\text { Oleyl methane } \\
\text { sulfonate }\end{array}$ & $\begin{array}{l}\operatorname{cccccccCC}=\operatorname{CCCCCCC} \\
\cos (C)(=0)=0\end{array}$ & & Sensitizer & & & & & ICCVAM (2013) & \\
\hline Oripavine & $\begin{array}{l}\mathrm{COC} 1=\mathrm{CC}=\mathrm{C} 2 \mathrm{C} 3 \mathrm{C} c 4 \mathrm{ccc} \\
(\mathrm{O}) \mathrm{c} 5 \mathrm{OC} 1 \mathrm{C} 2(\mathrm{CCN} 3 \mathrm{C}) \mathrm{c} 4 \\
5\end{array}$ & & Sensitizer & & & & & ICCVAM (2013) & \\
\hline Oxyfluorfen & $\begin{array}{l}\text { CCOc1cc(Oc2ccc(cc2Cl) } \\
C(F)(F) F) \operatorname{ccc} 1 N(=O)=O\end{array}$ & & Sensitizer & & & & & ICCVAM (2013) & \\
\hline Palmitoyl chloride & $\begin{array}{l}\operatorname{ccccccccccccccccC}(C \\
\mathrm{I})=0\end{array}$ & & Sensitizer & & & & & ICCVAM (2013) & \\
\hline Pyrogallol & Oc1cccc(0)c10 & & Sensitizer & & & & & ICCVAM (2013) & \\
\hline R-Carvoxime & $\begin{array}{l}\mathrm{CC}(=\mathrm{C}) \mathrm{C} 1 \mathrm{CC}=\mathrm{C}(\mathrm{C}) \mathrm{C}(\mathrm{C} 1) \\
=\mathrm{NO}\end{array}$ & & Sensitizer & & & & & ICCVAM (2013) & \\
\hline $\begin{array}{l}\text { REL-(3R,3aS,6aR)- } \\
\text { Hexahydrofuro[2,3- } \\
\text { b]furan-3-yl 4- } \\
\text { nitrophenyl carbonate }\end{array}$ & $\begin{array}{l}\mathrm{O}=\mathrm{C}(\mathrm{OC} 1 \mathrm{COC} 2 \mathrm{OCCC} 12 \\
\operatorname{OOc} 1 \mathrm{ccc}(\mathrm{cc} 1) \mathrm{N}(=\mathrm{O})=\mathrm{O}\end{array}$ & & Sensitizer & & & & & ICCVAM (2013) & \\
\hline $\begin{array}{l}\text { Sodium } 4-[(3,3,5- \\
\text { trimethylhexanoyl)oxy] } \\
\text { benzene-1-sulfonate }\end{array}$ & $\begin{array}{l}\mathrm{CC}(\mathrm{CC}(=0) 0 \mathrm{Oc} 1 \mathrm{ccc}(\mathrm{cc} 1) \\
\mathrm{S}(\mathrm{O})(=0)=0) \mathrm{CC}(\mathrm{C})(\mathrm{C}) \mathrm{C}\end{array}$ & & Sensitizer & & & & & ICCVAM (2013) & \\
\hline $\begin{array}{l}\text { Sodium bis(2- } \\
\text { methylpropyl)- } \\
\text { sulfanylidene- } \\
\text { sulfidophosphorane }\end{array}$ & $\mathrm{CC}(\mathrm{C}) \mathrm{CP}(\mathrm{S})(=\mathrm{S}) \mathrm{CC}(\mathrm{C}) \mathrm{C}$ & & Sensitizer & & & & & REACH (2008-2014) & \\
\hline Sodium ethyl xanthate & $\operatorname{ccOC}(S)=S$ & & Sensitizer & & & & & ICCVAM (2013) & \\
\hline Sodium lauroyl lactylate & $\begin{array}{l}\mathrm{CCCCCCCCCCCC}(=0) O C \\
(C) C(=O) O C(C) C(O)=O\end{array}$ & & Sensitizer & & & & & ICCVAM (2013) & \\
\hline Squalene & $\begin{array}{l}\mathrm{CC}(\mathrm{C})=\mathrm{CCCC}(\mathrm{C})=\mathrm{CCCC}( \\
\mathrm{C})=\mathrm{CCCC}=\mathrm{C}(\mathrm{C}) \mathrm{CCC}=\mathrm{C}(\mathrm{C} \\
) \mathrm{CCC}=\mathrm{C}(\mathrm{C}) \mathrm{C}\end{array}$ & & Sensitizer & & & & & ICCVAM (2013) & \\
\hline $\begin{array}{l}\text { Tetradecyl } \\
\text { chloroformate }\end{array}$ & $\begin{array}{l}\text { CCCCCCCCCCCCCCCOC( } \\
\mathrm{Cl})=0\end{array}$ & & Sensitizer & & & & & REACH (2008-2014) & \\
\hline $\begin{array}{l}\text { Toluene 2,4- } \\
\text { diisocyanate }\end{array}$ & $\begin{array}{l}\operatorname{Cc} 1 \operatorname{ccc}(\operatorname{cc} 1 \mathrm{~N}=\mathrm{C}=\mathrm{O}) \mathrm{N}=\mathrm{C} \\
=\mathrm{O}\end{array}$ & & Sensitizer & & & & & $\begin{array}{l}\text { ICCVAM }(2013)+ \\
\text { REACH }(2008-2014)\end{array}$ & \\
\hline $\begin{array}{l}\text { Toluene diamine } \\
\text { bismaleimide }\end{array}$ & $\begin{array}{l}\mathrm{Cc} 1 \operatorname{ccc}(\operatorname{cc} 1 \mathrm{~N} 1 \mathrm{C}(=0) \mathrm{C}= \\
\mathrm{CC} 1=0) \mathrm{N} 1 \mathrm{C}(=0) \mathrm{C}=\mathrm{CC} 1 \\
=0\end{array}$ & & Sensitizer & & & & & ICCVAM (2013) & \\
\hline Tridecane & CCCCCCCCCCCCCC & & Sensitizer & & & & & ICCVAM (2013) & \\
\hline Trifluralin & $\begin{array}{l}\text { CCCN(CCC)c1c(cc(cc1N } \\
(=0)=O) C(F)(F) F) N(=0) \\
=0\end{array}$ & & Sensitizer & & & & & ICCVAM (2013) & \\
\hline
\end{tabular}




\begin{tabular}{|c|c|c|c|c|c|c|c|c|c|}
\hline Compound name & SMILES & $\begin{array}{l}\text { Human } \\
\text { result }\end{array}$ & $\begin{array}{l}\text { LLNA } \\
\text { result }\end{array}$ & DPRA & KeratinoSens & h-CLAT & $\begin{array}{l}\text { Human } \\
\text { reference }\end{array}$ & LLNA reference & Non-animal reference \\
\hline $\begin{array}{l}\text { Trimethylhexamine } \\
\text { diamine }\end{array}$ & $\mathrm{CC}(\mathrm{CCCCN}) \mathrm{C}(\mathrm{C})(\mathrm{C}) \mathrm{N}$ & & Sensitizer & & & & & ICCVAM (2013) & \\
\hline Trinitrochlorobenzene & $\begin{array}{l}\text { Clc1ccc(c(c1N }(=0)=0) \\
\mathrm{N}(=0)=0) \mathrm{N}(=0)=0\end{array}$ & & Sensitizer & & & & & ICCVAM (2013) & \\
\hline Triphenyl phosphite & $\begin{array}{l}\mathrm{O}(\mathrm{P}(\mathrm{Oc} 1 \mathrm{ccccc} 1) \mathrm{Oc} 1 \mathrm{ccc} \\
\mathrm{cc} 1) \mathrm{c} 1 \mathrm{ccccc} 1\end{array}$ & & Sensitizer & & & & & REACH (2008-2014) & \\
\hline Triton X-100 & $\begin{array}{l}\mathrm{CC}(\mathrm{C})(\mathrm{C}) \mathrm{CC}(\mathrm{C})(\mathrm{C}) \mathrm{c} 1 \mathrm{ccc}( \\
\mathrm{OCCO}) \mathrm{cc} 1\end{array}$ & & Sensitizer & & & & & ICCVAM (2013) & \\
\hline Veratraldehyde & $\operatorname{coc} 1 \mathrm{ccc}(\mathrm{C}=0) \operatorname{cc} 10 \mathrm{C}$ & & Sensitizer & & & & & ICCVAM (2013) & \\
\hline $\begin{array}{l}\text { [(1R,4aR,4bS,10aR)- } \\
\text { 1,4a-dimethyl-7- } \\
\text { propan-2-yl- } \\
2,3,4,4 b, 5,6,7,8,8 a, 9,10 \\
\text { 10a- } \\
\text { dodecahydrophenanthr } \\
\text { en-1-yl]methanol }\end{array}$ & $\begin{array}{l}\mathrm{CC}(\mathrm{C}) \mathrm{C} 1 \mathrm{CCC} 2 \mathrm{C}(\mathrm{CCC} 3 \mathrm{C}( \\
\mathrm{C})(\mathrm{CO}) \mathrm{CCCC} 23 \mathrm{C}) \mathrm{C} 1\end{array}$ & & Sensitizer & & & & & REACH (2008-2014) & \\
\hline $\begin{array}{l}\text { [(1S,4S)-1,7,7-trimethyl- } \\
6 \text { - } \\
\text { bicyclo[2.2.1]heptanyl] } \\
\text { prop-2-enoate }\end{array}$ & $\begin{array}{l}\mathrm{CC} 1(\mathrm{C}) \mathrm{C} 2 \mathrm{CCC} 1(\mathrm{C}) \mathrm{C}(\mathrm{C} 2) \\
\mathrm{OC}(=\mathrm{O}) \mathrm{C}=\mathrm{C}\end{array}$ & & Sensitizer & & & & & REACH (2008-2014) & \\
\hline $\begin{array}{l}\text { [2,2-dimethyl-3- } \\
\text { (morpholin-4- } \\
\text { yl)propylidene](6-\{[2,2- } \\
\text { dimethyl-3-(morpholin- } \\
4- \\
\text { yl)propylidene]amino }\} \text { h } \\
\text { exyl)amine }\end{array}$ & $\begin{array}{l}\mathrm{CC}(\mathrm{C})(\mathrm{CN} 1 \mathrm{CCOCC} 1) \mathrm{C}= \\
\mathrm{NCCCCCN}=\mathrm{CC}(\mathrm{C})(\mathrm{C}) \mathrm{C} \\
\mathrm{N} 1 \mathrm{CCOCC} 1\end{array}$ & & Sensitizer & & & & & REACH (2008-2014) & \\
\hline $\begin{array}{l}\text { [2,2-dimethyl-3- } \\
\text { (morpholin-4- } \\
\text { yl)propylidene][(5-\{[2,2- } \\
\text { dimethyl-3-(morpholin- } \\
4- \\
\text { yl)propylidene]amino\}- } \\
\text { 1,3,3- } \\
\text { trimethylcyclohexyl)me } \\
\text { thyl]amine }\end{array}$ & $\begin{array}{l}\mathrm{CC}(\mathrm{C})(\mathrm{CN} 1 \mathrm{CCOCC} 1) \mathrm{C}= \\
\mathrm{NCC1}(\mathrm{C}) \mathrm{CC}(\mathrm{CC}(\mathrm{C})(\mathrm{C}) \mathrm{C} 1 \\
) \mathrm{N}=\mathrm{CC}(\mathrm{C})(\mathrm{C}) \mathrm{CN} 1 \mathrm{CCOCC} \\
1\end{array}$ & & Sensitizer & & & & & REACH (2008-2014) & \\
\hline $\begin{array}{l}\text { [2-hydroxy-3-[4-(2- } \\
\text { hydroxy-3-prop-2- } \\
\text { enoyloxypropoxy)butox } \\
\text { y]propyl] prop-2-enoate }\end{array}$ & $\begin{array}{l}\mathrm{OC}(\operatorname{COCCCCOCC}(O) \operatorname{CO} \\
\mathrm{C}(=\mathrm{O}) \mathrm{C}=\mathrm{C}) \operatorname{coc}(=\mathrm{O}) \mathrm{C}=\mathrm{C}\end{array}$ & & Sensitizer & & & & & REACH (2008-2014) & \\
\hline $\begin{array}{l}\text { [3-(2-methoxy-5- } \\
\text { methylphenyl)-3- } \\
\text { phenylpropyl]bis(propa } \\
\text { n-2-yl)amine }\end{array}$ & $\begin{array}{l}\operatorname{COc} 1 \operatorname{ccc}(\mathrm{C}) \operatorname{cc} 1 \mathrm{C}(\mathrm{CCN}(\mathrm{C} \\
(\mathrm{C}) \mathrm{C}) \mathrm{C}(\mathrm{C}) \mathrm{C}) \mathrm{c} 1 \mathrm{ccccc} 1\end{array}$ & & Sensitizer & & & & & REACH (2008-2014) & \\
\hline
\end{tabular}




\begin{tabular}{|c|c|c|c|c|c|c|c|c|c|}
\hline Compound name & SMILES & $\begin{array}{l}\text { Human } \\
\text { result }\end{array}$ & $\begin{array}{l}\text { LLNA } \\
\text { result }\end{array}$ & DPRA & KeratinoSens & h-CLAT & $\begin{array}{l}\text { Human } \\
\text { reference }\end{array}$ & LLNA reference & Non-animal reference \\
\hline $\begin{array}{l}\text { bis(2-methylpropyl) } \\
\text { cyclohexane-1,2- } \\
\text { dicarboxylate }\end{array}$ & $\begin{array}{l}\mathrm{CC}(\mathrm{C}) \mathrm{COC}(=0) \mathrm{C} 1 \mathrm{CCCC} \\
\mathrm{C} 1 \mathrm{C}(=0) \mathrm{OCC}(\mathrm{C}) \mathrm{C}\end{array}$ & & Sensitizer & & & & & REACH (2008-2014) & \\
\hline $\begin{array}{l}\text { bis(3-methylbutyl) } \\
\text { benzene-1,2- } \\
\text { dicarboxylate }\end{array}$ & $\begin{array}{l}\mathrm{CC}(\mathrm{C}) \mathrm{CCOC}(=0) \mathrm{c} 1 \mathrm{ccccc} \\
1 \mathrm{C}(=0) \mathrm{OCCC}(\mathrm{C}) \mathrm{C}\end{array}$ & & Sensitizer & & & & & REACH (2008-2014) & \\
\hline $\begin{array}{l}\text { bis(8-methylnonyl) } \\
\text { phenyl phosphite }\end{array}$ & $\begin{array}{l}\mathrm{CC}(\mathrm{C}) \mathrm{CCCCCCCOP}(\mathrm{OCC} \\
\mathrm{CCCCCC}(\mathrm{C}) \mathrm{C}) \text { Oc1 } 1 \mathrm{ccccc} 1\end{array}$ & & Sensitizer & & & & & REACH (2008-2014) & \\
\hline $\begin{array}{l}\text { but-1-ene; (E)-but-2- } \\
\text { ene }\end{array}$ & $\mathrm{CCC}=\mathrm{C}$ & & Sensitizer & & & & & REACH (2008-2014) & \\
\hline $\begin{array}{l}\text { butyl N- } \\
\text { [(butylsulfanyl)methane } \\
\text { thioyl]carbamate }\end{array}$ & $\begin{array}{l}\mathrm{CCCCOC}(=0) \mathrm{NC}(=\mathrm{S}) \mathrm{SCC} \\
\mathrm{CC}\end{array}$ & & Sensitizer & & & & & REACH (2008-2014) & \\
\hline $\begin{array}{l}\text { cyclohexyl 2- } \\
\text { methylprop-2-enoate }\end{array}$ & $\begin{array}{l}\mathrm{CC}(=\mathrm{C}) \mathrm{C}(=0) \mathrm{OC} 1 \mathrm{CCCCC} \\
1\end{array}$ & & Sensitizer & & & & & REACH (2008-2014) & \\
\hline $\begin{array}{l}\text { di(phenyl)phosphoryl- } \\
(2,4,6- \\
\text { trimethylphenyl)metha } \\
\text { none }\end{array}$ & $\begin{array}{l}\mathrm{CC} 1=\mathrm{CC}(=\mathrm{C}) \mathrm{C}(=\mathrm{C}(\mathrm{O}) \mathrm{P}(= \\
\mathrm{O})(\mathrm{c} 2 \operatorname{ccccc} 2) \operatorname{c} 2 \operatorname{ccccc} 2) \\
\mathrm{C}(\mathrm{C})=\mathrm{C} 1\end{array}$ & & Sensitizer & & & & & REACH (2008-2014) & \\
\hline $\begin{array}{l}\text { dibutyl benzene-1,4- } \\
\text { dicarboxylate }\end{array}$ & $\begin{array}{l}\text { CCCCOC }(=0) \operatorname{c1ccc}(\operatorname{cc} 1) \\
\mathrm{C}(=0) \mathrm{OCCCC}\end{array}$ & & Sensitizer & & & & & REACH (2008-2014) & \\
\hline $\begin{array}{l}\text { diprop-2-enyl benzene- } \\
\text { 1,2-dicarboxylate }\end{array}$ & $\begin{array}{l}\mathrm{C}=\mathrm{CCOC}(=0) \operatorname{c} 1 \mathrm{ccccc} 1 \mathrm{C} \\
(=0) \mathrm{OCC}=\mathrm{C}\end{array}$ & & Sensitizer & & & & & REACH (2008-2014) & \\
\hline $\begin{array}{l}\text { ethenyl 2- } \\
\text { ethylhexanoate }\end{array}$ & $\mathrm{CCCCC}(\mathrm{CC}) \mathrm{C}(=\mathrm{O}) \mathrm{OC}=\mathrm{C}$ & & Sensitizer & & & & & REACH (2008-2014) & \\
\hline $\begin{array}{l}\text { ethoxymethoxycyclodo } \\
\text { decane }\end{array}$ & $\begin{array}{l}\text { CCOCOC1CCCCCCCCCC } \\
\mathrm{C} 1\end{array}$ & & Sensitizer & & & & & REACH (2008-2014) & \\
\hline $\begin{array}{l}\text { formaldehyde; 2-(2- } \\
\text { hydroxyethylamino)eth } \\
\text { anol; 2-methyloxirane; } \\
\text { 2-nonylphenol }\end{array}$ & cCCCCCCCCc1ccccc10 & & Sensitizer & & & & & REACH (2008-2014) & \\
\hline $\begin{array}{l}\text { hept-4-en-2-yl 2- } \\
\text { hydroxybenzoate }\end{array}$ & $\begin{array}{l}\mathrm{CCC}=\mathrm{CCC}(\mathrm{C}) \mathrm{OC}(=0) \mathrm{c} 1 \mathrm{c} \\
\operatorname{cccc} 10\end{array}$ & & Sensitizer & & & & & REACH (2008-2014) & \\
\hline $\begin{array}{l}\text { hex-3-enyl 2- } \\
\text { hydroxybenzoate }\end{array}$ & $\begin{array}{l}\mathrm{CCC}=\mathrm{CCCOC}(=0) \mathrm{c} 1 \mathrm{cccc} \\
\mathrm{c} 10\end{array}$ & & Sensitizer & & & & & REACH (2008-2014) & \\
\hline hex-3-yne-2,5-diol & $\mathrm{CC}(\mathrm{O}) \mathrm{C \# CC}(\mathrm{C}) \mathrm{O}$ & & Sensitizer & & & & & REACH (2008-2014) & \\
\hline $\begin{array}{l}\mathrm{m}- \\
\text { Phenylenebis(methyla } \\
\text { mine) }\end{array}$ & $\mathrm{NCc1} \operatorname{cccc}(\mathrm{CN}) \mathrm{c1}$ & & Sensitizer & & & & & $\begin{array}{l}\text { ICCVAM (2013) + } \\
\text { REACH }(2008-2014)\end{array}$ & \\
\hline n-Methyl-n-nitrosourea & $\mathrm{CN}(\mathrm{N}=\mathrm{O}) \mathrm{C}(\mathrm{N})=\mathrm{O}$ & & Sensitizer & & & & & ICCVAM (2013) & \\
\hline oxolane-2,5-dione & $\mathrm{O}=\mathrm{C} 1 \mathrm{CCC}(=0) \mathrm{O} 1$ & & Sensitizer & & & & & REACH (2008-2014) & \\
\hline $\begin{array}{l}\text { p-chlorophenethylic } \\
\text { alcohol }\end{array}$ & OCCc1ccc(Cl)cc1 & & Sensitizer & & & & & ICCVAM (2013) & \\
\hline
\end{tabular}




\begin{tabular}{|c|c|c|c|c|c|c|c|c|c|}
\hline Compound name & SMILES & $\begin{array}{l}\text { Human } \\
\text { result }\end{array}$ & $\begin{array}{l}\text { LLNA } \\
\text { result }\end{array}$ & DPRA & KeratinoSens & h-CLAT & $\begin{array}{l}\text { Human } \\
\text { reference }\end{array}$ & LLNA reference & Non-animal reference \\
\hline $\begin{array}{l}\text { p-tert-Butylphenyl 1- } \\
\text { (2,3-epoxy)propyl ether }\end{array}$ & $\begin{array}{l}\mathrm{CC}(\mathrm{C})(\mathrm{C}) \mathrm{c} 1 \mathrm{ccc}(\mathrm{OCC} 2 \mathrm{CO} \\
2) \mathrm{cc} 1\end{array}$ & & Sensitizer & & & & & $\begin{array}{l}\text { ICCVAM }(2013)+ \\
\text { REACH }(2008-2014)\end{array}$ & \\
\hline $\begin{array}{l}\text { potassium 3- } \\
\text { methylbutoxymethane } \\
\text { dithioate }\end{array}$ & $\mathrm{CC}(\mathrm{C}) \mathrm{CCOC}(\mathrm{S})=\mathrm{S}$ & & Sensitizer & & & & & REACH (2008-2014) & \\
\hline $\begin{array}{l}\text { tert-butyl } \\
\text { benzenecarboperoxoat } \\
\text { e }\end{array}$ & $\begin{array}{l}\mathrm{CC}(\mathrm{C})(\mathrm{C}) \mathrm{OOC}(=0) \mathrm{c} 1 \mathrm{ccc} \\
\mathrm{cc1}\end{array}$ & & Sensitizer & & & & & REACH (2008-2014) & \\
\hline $\begin{array}{l}\text { tetradecoxycarbonyloxy } \\
\text { tetradecyl carbonate }\end{array}$ & $\begin{array}{l}\text { CCCCCCCCCCCCCCOC( } \\
=0) \text { OOC }(=0) \text { OcCCCCC } \\
\text { cCCCCCCC }\end{array}$ & & Sensitizer & & & & & REACH (2008-2014) & \\
\hline trans-Anethol & $\operatorname{coc} 1 \mathrm{ccc}(\mathrm{C}=\mathrm{CC}) \mathrm{cc} 1$ & & Sensitizer & & & & & $\begin{array}{l}\text { ICCVAM (2013) + } \\
\text { REACH }(2008-2014)\end{array}$ & \\
\hline $\begin{array}{l}\text { tri(propan-2-yl)silyl } \\
\text { prop-2-enoate }\end{array}$ & $\begin{array}{l}\mathrm{CC}(\mathrm{C})[\mathrm{Si}](\mathrm{OC}(=\mathrm{O}) \mathrm{C}=\mathrm{C})( \\
\mathrm{C}(\mathrm{C}) \mathrm{C}) \mathrm{C}(\mathrm{C}) \mathrm{C}\end{array}$ & & Sensitizer & & & & & REACH (2008-2014) & \\
\hline $\begin{array}{l}\text { trimethyl[3- } \\
\text { (octadecyloxy)propyl]az } \\
\text { anium }\end{array}$ & $\begin{array}{l}\text { CCCCCCCCCCCCCCCCC } \\
\operatorname{cocCC}[N+](C)(C) C\end{array}$ & & Sensitizer & & & & & REACH (2008-2014) & \\
\hline $\begin{array}{l}\text { tris(11-methyldodecyl) } \\
\text { phosphite }\end{array}$ & $\begin{array}{l}\text { CC(C)CCCCCCCCCCOP( } \\
\text { OCCCCCCCCCCC(C)C)O } \\
\text { CCCCCCCCCCC(C)C }\end{array}$ & & Sensitizer & & & & & REACH (2008-2014) & \\
\hline $\begin{array}{l}\text { tris(8-methylnonyl) } \\
\text { phosphite }\end{array}$ & $\begin{array}{l}\mathrm{CC}(\mathrm{C}) \mathrm{CCCCCCCOP}(\mathrm{OCC} \\
\mathrm{CCCCCC}(\mathrm{C}) \mathrm{C}) \mathrm{OCCCCCC} \\
\mathrm{CC}(\mathrm{C}) \mathrm{C}\end{array}$ & & Sensitizer & & & & & REACH (2008-2014) & \\
\hline $\begin{array}{l}\text { tris(propan-2-yl)silyl 2- } \\
\text { methylprop-2-enoate }\end{array}$ & $\begin{array}{l}\mathrm{CC}(\mathrm{C})[\mathrm{Si}](\mathrm{OC}(=\mathrm{O}) \mathrm{C}(\mathrm{C})= \\
\mathrm{C})(\mathrm{C}(\mathrm{C}) \mathrm{C}) \mathrm{C}(\mathrm{C}) \mathrm{C}\end{array}$ & & Sensitizer & & & & & REACH (2008-2014) & \\
\hline $\begin{array}{l}\text { tris[4- } \\
\text { (diethylamino)phenyl] } \\
\text { methylium }\end{array}$ & $\begin{array}{l}\operatorname{CCN}(\mathrm{CC}) \mathrm{c} 1 \mathrm{ccc}(\mathrm{cc} 1)[\mathrm{C}+] \\
(\mathrm{c} 1 \mathrm{ccc}(\mathrm{cc} 1) \mathrm{N}(\mathrm{CC}) \mathrm{CC}) \mathrm{c1} \\
\operatorname{ccc}(\mathrm{cc} 1) \mathrm{N}(\mathrm{CC}) \mathrm{CC}\end{array}$ & & Sensitizer & & & & & REACH (2008-2014) & \\
\hline $\begin{array}{l}\alpha \text {-(p-toluenesulfonyl)-4- } \\
\text { fluorobenzyliso-nitrile }\end{array}$ & $\begin{array}{l}\mathrm{Cc} 1 \mathrm{ccc}(\mathrm{cc} 1) \mathrm{S}(=\mathrm{O})(=\mathrm{O}) \mathrm{C} \\
([\mathrm{N}+] \# \mathrm{C}) \mathrm{c} 1 \mathrm{ccc}(\mathrm{F}) \mathrm{ccc} 1\end{array}$ & & Sensitizer & & & & & ICCVAM (2013) & \\
\hline $\begin{array}{l}\alpha \text {-Butyl cinnamic } \\
\text { aldehyde }\end{array}$ & $\begin{array}{l}\operatorname{ccccC}(C=0)=\operatorname{Cc} 1 \operatorname{ccccc} \\
1\end{array}$ & & Sensitizer & & & & & ICCVAM (2013) & \\
\hline $\begin{array}{l}\alpha \text {-Methyl-1,3- } \\
\text { benzodioxole-5- } \\
\text { propionaldehyde }\end{array}$ & $\begin{array}{l}\mathrm{CC}(\mathrm{Cc} 1 \mathrm{ccc} 2 \mathrm{OCO} 2 \mathrm{c} 1) \mathrm{C} \\
=\mathrm{O}\end{array}$ & & Sensitizer & & & & & ICCVAM (2013) & \\
\hline$\alpha$-Phellandrene & $\mathrm{CC}(\mathrm{C}) \mathrm{C} 1 \mathrm{CC}=\mathrm{C}(\mathrm{C}) \mathrm{C}=\mathrm{C} 1$ & & Sensitizer & & & & & ICCVAM (2013) & \\
\hline$\alpha$-Terpinene & $\mathrm{CC}(\mathrm{C}) \mathrm{C} 1=\mathrm{CC}=\mathrm{C}(\mathrm{C}) \mathrm{CC} 1$ & & Sensitizer & & & & & ICCVAM (2013) & \\
\hline$\beta$-Phellandrene & $\mathrm{CC}(\mathrm{C}) \mathrm{C} 1 \mathrm{CCC}(=\mathrm{C}) \mathrm{C}=\mathrm{C} 1$ & & Sensitizer & & & & & ICCVAM (2013) & \\
\hline $\begin{array}{l}\beta- \\
\text { Phenylcinnamaldehyde }\end{array}$ & $\begin{array}{l}\mathrm{O}=\mathrm{CC}=\mathrm{C}(\mathrm{c} 1 \mathrm{ccccc} 1) \mathrm{c} 1 \mathrm{cc} \\
\mathrm{ccc} 1\end{array}$ & & Sensitizer & & & & & ICCVAM (2013) & \\
\hline$\beta$-Propiolactone & $\mathrm{O}=\mathrm{C} 1 \mathrm{CCO} 1$ & & Sensitizer & & & & & ICCVAM (2013) & \\
\hline
\end{tabular}




\begin{tabular}{|c|c|c|c|c|c|c|c|c|c|}
\hline Compound name & SMILES & $\begin{array}{l}\text { Human } \\
\text { result }\end{array}$ & $\begin{array}{l}\text { LLNA } \\
\text { result }\end{array}$ & DPRA & KeratinoSens & h-CLAT & $\begin{array}{l}\text { Human } \\
\text { reference }\end{array}$ & LLNA reference & Non-animal reference \\
\hline 1-lodohexane & $\mathrm{CCCCCCl}$ & & $\begin{array}{l}\text { Non- } \\
\text { sensitizer }\end{array}$ & Positive & Positive & Positive & & ICCVAM (2013) & Urbisch et al. (2015) \\
\hline 2-Acetylcyclohexanone & $\mathrm{CC}(=0) \mathrm{C} 1=\mathrm{C}(0) \mathrm{CCCC} 1$ & & $\begin{array}{l}\text { Non- } \\
\text { sensitizer }\end{array}$ & Positive & Positive & Positive & & ICCVAM (2013) & Urbisch et al. (2015) \\
\hline 2-Fluoro-5-nitroaniline & $\operatorname{Nc} 1 \operatorname{cc}(\operatorname{ccc} 1 F) N(=0)=0$ & & $\begin{array}{l}\text { Non- } \\
\text { sensitizer }\end{array}$ & Positive & Positive & Negative & & ICCVAM (2013) & Urbisch et al. (2015) \\
\hline Furil & $\begin{array}{l}\mathrm{O}=\mathrm{C}(\mathrm{C}(=0) \mathrm{c} 1 \mathrm{ccco} 1) \mathrm{c} 1 \mathrm{c} \\
\mathrm{cco1}\end{array}$ & & $\begin{array}{l}\text { Non- } \\
\text { sensitizer }\end{array}$ & Positive & Positive & Negative & & ICCVAM (2013) & Urbisch et al. (2015) \\
\hline $\begin{array}{l}\text { Propanoic acid, 3- } \\
\text { bromo, methyl ester }\end{array}$ & $\mathrm{COC}(=\mathrm{O}) \mathrm{CCBr}$ & & $\begin{array}{l}\text { Non- } \\
\text { sensitizer }\end{array}$ & Positive & Positive & Negative & & ICCVAM (2013) & Urbisch et al. (2015) \\
\hline 1-Bromobutane & $\mathrm{CCCCBr}$ & & $\begin{array}{l}\text { Non- } \\
\text { sensitizer }\end{array}$ & Positive & Negative & Positive & & ICCVAM (2013) & Urbisch et al. (2015) \\
\hline $\begin{array}{l}\text { 3- } \\
\text { Phenoxypropiononitrile }\end{array}$ & N\#CCCOc1ccccc1 & & $\begin{array}{l}\text { Non- } \\
\text { sensitizer }\end{array}$ & Positive & Negative & Positive & & ICCVAM (2013) & Urbisch et al. (2015) \\
\hline $\begin{array}{l}\mathrm{N}, \mathrm{N} \text {-diethyl-m- } \\
\text { toluamide }\end{array}$ & $\begin{array}{l}\operatorname{CCN}(\mathrm{CC}) \mathrm{C}(=0) \operatorname{c1} \operatorname{cccc}(\mathrm{C} \\
)_{\mathrm{c} 1}\end{array}$ & & $\begin{array}{l}\text { Non- } \\
\text { sensitizer }\end{array}$ & Positive & Negative & Negative & & ICCVAM (2013) & Urbisch et al. (2015) \\
\hline 2-Butoxyethyl acetate & $\operatorname{ccccoccoc}(C)=0$ & & $\begin{array}{l}\text { Non- } \\
\text { sensitizer }\end{array}$ & Positive & Negative & & & ICCVAM (2013) & Urbisch et al. (2015) \\
\hline $\begin{array}{l}\text { 3-Chloro-p- } \\
\text { anisaldehyde }\end{array}$ & $\operatorname{coc} 1 \mathrm{ccc}(\mathrm{C}=0) \operatorname{cc} 1 \mathrm{Cl}$ & & $\begin{array}{l}\text { Non- } \\
\text { sensitizer }\end{array}$ & Positive & Negative & & & ICCVAM (2013) & Urbisch et al. (2015) \\
\hline $\begin{array}{l}\text { 4- } \\
\text { Carboxyphenylacetate }\end{array}$ & $\begin{array}{l}\mathrm{CC}(=0) \operatorname{Oc} 1 \mathrm{ccc}(\mathrm{cc} 1) \mathrm{C}(0 \\
)=0\end{array}$ & & $\begin{array}{l}\text { Non- } \\
\text { sensitizer }\end{array}$ & Positive & Negative & & & $\begin{array}{l}\text { J Appl Toxicol, v } \\
33(11), 1353-64,2013 .\end{array}$ & Urbisch et al. (2015) \\
\hline 4-Chloroaniline & $\mathrm{Nc} 1 \mathrm{ccc}(\mathrm{Cl}) \mathrm{cc} 1$ & & $\begin{array}{l}\text { Non- } \\
\text { sensitizer }\end{array}$ & Negative & Positive & Positive & & ICCVAM (2013) & Urbisch et al. (2015) \\
\hline $\begin{array}{l}\text { Benzene, 1-methoxy-4- } \\
\text { methyl-2-nitro }\end{array}$ & $\begin{array}{l}\operatorname{COc} 1 \operatorname{ccc}(\mathrm{C}) \operatorname{cc} 1 \mathrm{~N}(=\mathrm{O})= \\
\mathrm{O}\end{array}$ & & $\begin{array}{l}\text { Non- } \\
\text { sensitizer }\end{array}$ & Negative & Positive & Positive & & ICCVAM (2013) & Urbisch et al. (2015) \\
\hline $\begin{array}{l}\text { 1,2- } \\
\text { Benzenedicarboxylic } \\
\text { acid, dibutyl ester }\end{array}$ & $\begin{array}{l}\operatorname{ccCCOC}(=0) \operatorname{c1ccccc} 1 \mathrm{C} \\
(=0) \mathrm{OCCCC}\end{array}$ & & $\begin{array}{l}\text { Non- } \\
\text { sensitizer }\end{array}$ & Negative & Positive & Negative & & ICCVAM (2013) & Urbisch et al. (2015) \\
\hline Butyl benzyl phthalate & $\begin{array}{l}\text { CCCCOC }(=0) \operatorname{c1ccccc} 1 \mathrm{C} \\
(=0) \mathrm{OCc} 1 \mathrm{ccccc} 1\end{array}$ & & $\begin{array}{l}\text { Non- } \\
\text { sensitizer }\end{array}$ & Negative & Positive & Negative & & ICCVAM (2013) & Urbisch et al. (2015) \\
\hline Ethyl benzoylacetate & $\begin{array}{l}\mathrm{CCOC}(=0) \mathrm{CC}(=0) \mathrm{c} 1 \mathrm{ccc} \\
\mathrm{cc} 1\end{array}$ & & $\begin{array}{l}\text { Non- } \\
\text { sensitizer }\end{array}$ & Negative & Positive & Negative & & ICCVAM (2013) & Urbisch et al. (2015) \\
\hline $\begin{array}{l}\text { Methyl 4- } \\
\text { hydroxybenzoate } \\
\text { (methylparaben) }\end{array}$ & $\operatorname{coc}(=0) \operatorname{cocc}(0) \operatorname{cc} 1$ & & $\begin{array}{l}\text { Non- } \\
\text { sensitizer }\end{array}$ & Negative & Positive & & & ICCVAM (2013) & Urbisch et al. (2015) \\
\hline Clofibrate & $\begin{array}{l}\mathrm{CCOC}(=0) \mathrm{C}(\mathrm{C})(\mathrm{C}) 0 \mathrm{c} 1 \mathrm{cc} \\
\mathrm{c}(\mathrm{Cl}) \mathrm{cc} 1\end{array}$ & & $\begin{array}{l}\text { Non- } \\
\text { sensitizer }\end{array}$ & Negative & Negative & Positive & & ICCVAM (2013) & Urbisch et al. (2015) \\
\hline Nonanoic acid & $\operatorname{ccccccccC}(0)=0$ & & $\begin{array}{l}\text { Non- } \\
\text { sensitizer }\end{array}$ & Negative & Negative & Positive & & REACH (2008-2014) & Urbisch et al. (2015) \\
\hline 4-Hydroxybenzoic acid & $\mathrm{OC}(=0) \mathrm{c} 1 \mathrm{ccc}(\mathrm{O}) \mathrm{cc} 1$ & & $\begin{array}{l}\text { Non- } \\
\text { sensitizer }\end{array}$ & Negative & Negative & Negative & & $\begin{array}{l}\text { ICCVAM }(2013)+ \\
\text { REACH }(2008-2014)\end{array}$ & Urbisch et al. (2015) \\
\hline Saccharin & $\begin{array}{l}\mathrm{O}=\mathrm{C} 1 \mathrm{NS}(=\mathrm{O})(=\mathrm{O}) \mathrm{c} 2 \mathrm{ccc} \\
\mathrm{cc} 12\end{array}$ & & $\begin{array}{l}\text { Non- } \\
\text { sensitizer }\end{array}$ & Negative & Negative & Negative & & ICCVAM (2013) & Urbisch et al. (2015) \\
\hline
\end{tabular}




\begin{tabular}{|c|c|c|c|c|c|c|c|c|c|}
\hline Compound name & SMILES & $\begin{array}{l}\text { Human } \\
\text { result }\end{array}$ & $\begin{array}{l}\text { LLNA } \\
\text { result }\end{array}$ & DPRA & KeratinoSens & h-CLAT & $\begin{array}{l}\text { Human } \\
\text { reference }\end{array}$ & LLNA reference & Non-animal reference \\
\hline Octanenitrile & CCCCCCCC\#N & & $\begin{array}{l}\text { Non- } \\
\text { sensitizer }\end{array}$ & Negative & Negative & & & ICCVAM (2013) & Urbisch et al. (2015) \\
\hline Vinylidene dichloride & $\mathrm{ClC}(\mathrm{Cl})=\mathrm{C}$ & & $\begin{array}{l}\text { Non- } \\
\text { sensitizer }\end{array}$ & Negative & Negative & & & $\begin{array}{l}\text { ICCVAM }(2013)+ \\
\operatorname{REACH}(2008-2014)\end{array}$ & Urbisch et al. (2015) \\
\hline (+)-Estrone & $\begin{array}{l}\mathrm{CC} 12 \mathrm{CCC} 3 \mathrm{C}(\mathrm{CCc} 4 \mathrm{cc}(0) \\
\operatorname{ccc} 34) \mathrm{C} 1 \mathrm{CCC} 2=0\end{array}$ & & $\begin{array}{l}\text { Non- } \\
\text { sensitizer }\end{array}$ & & & & & REACH (2008-2014) & \\
\hline $\begin{array}{l}\text { (16- } \beta \text { )-21-(acetyloxy)- } \\
\text { 17-hydroxy-16- } \\
\text { methylpregna- } \\
\text { 1,4,9(11)-triene-3,20- } \\
\text { dione }\end{array}$ & $\begin{array}{l}\mathrm{CC} 1 \mathrm{CC} 2 \mathrm{C} 3 \mathrm{CCC} 4=\mathrm{CC}(=\mathrm{O} \\
) \mathrm{C}=\mathrm{CC} 4(\mathrm{C}) \mathrm{C} 3=\mathrm{CCC} 2(\mathrm{C}) \mathrm{C} \\
1(\mathrm{O}) \mathrm{C}(=0) \operatorname{COC}(\mathrm{C})=0\end{array}$ & & $\begin{array}{l}\text { Non- } \\
\text { sensitizer }\end{array}$ & & & & & ICCVAM (2013) & \\
\hline $\begin{array}{l}\text { (1R,2S,3R,4R,6S,7S,8S,1 } \\
\text { 4R)-3-Hydroxy-2,4,7,14- } \\
\text { tetramethyl-9-oxo-4- } \\
\text { vinyltricyclo[5.4.3.01,8] } \\
\text { tetradec-6-yl } \\
\text { [(methylsulfonyl)oxy]ac } \\
\text { etate }\end{array}$ & $\begin{array}{l}\mathrm{CC1CCC23CCC}(=0) C 2 C \\
1(C) C(C C(C)(C=C) C(O) C \\
3 C) O C(=0) \cos (C)(=0)= \\
0\end{array}$ & & $\begin{array}{l}\text { Non- } \\
\text { sensitizer }\end{array}$ & & & & & ICCVAM (2013) & \\
\hline $\begin{array}{l}\text { (1R,2S,5R)-5-methyl-2- } \\
\text { propan-2-ylcyclohexan- } \\
\text { 1-ol }\end{array}$ & $\mathrm{CC}(\mathrm{C}) \mathrm{C} 1 \mathrm{CCC}(\mathrm{C}) \mathrm{CC} 10$ & & $\begin{array}{l}\text { Non- } \\
\text { sensitizer }\end{array}$ & & & & & REACH (2008-2014) & \\
\hline $\begin{array}{l}\text { (1R,4R)-4-Isopropenyl- } \\
\text { 1-methyl-2- } \\
\text { methylenecyclohexane }\end{array}$ & $\mathrm{CC} 1 \mathrm{CCC}(\mathrm{CC} 1=\mathrm{C}) \mathrm{C}(\mathrm{C})=\mathrm{C}$ & & $\begin{array}{l}\text { Non- } \\
\text { sensitizer }\end{array}$ & & & & & ICCVAM (2013) & \\
\hline $\begin{array}{l}\text { (1Z)-1-[(2,4- } \\
\text { dinitrophenyl)hydraziny } \\
\text { lidene]naphthalen-2- } \\
\text { one }\end{array}$ & $\begin{array}{l}\mathrm{O}=\mathrm{C} 1 \mathrm{C}=\mathrm{Cc} 2 \operatorname{cccccc} 2 \mathrm{C} 1= \\
\mathrm{NNc} 1 \operatorname{ccc}(\operatorname{cc} 1 \mathrm{~N}(=\mathrm{O})=\mathrm{O}) \\
\mathrm{N}(=\mathrm{O})=\mathrm{O}\end{array}$ & & $\begin{array}{l}\text { Non- } \\
\text { sensitizer }\end{array}$ & & & & & REACH (2008-2014) & \\
\hline $\begin{array}{l}\text { (2,5-dioxoimidazolidin- } \\
\text { 4-yl)urea }\end{array}$ & $\begin{array}{l}\mathrm{NC}(=0) \mathrm{NC} 1 \mathrm{NC}(=0) \mathrm{NC1} \\
=0\end{array}$ & & $\begin{array}{l}\text { Non- } \\
\text { sensitizer }\end{array}$ & & & & & REACH (2008-2014) & \\
\hline $\begin{array}{l}\text { (2-Bromo-5- } \\
\text { propoxyphenyl)(2- } \\
\text { hydroxy-4- } \\
\text { methoxyphenyl)- } \\
\text { methanone }\end{array}$ & $\begin{array}{l}\mathrm{CCCOc} 1 \mathrm{ccc}(\mathrm{Br}) \mathrm{c}(\mathrm{c} 1) \mathrm{C}(= \\
0) \operatorname{cocc}(\mathrm{OC}) \operatorname{cc} 10\end{array}$ & & $\begin{array}{l}\text { Non- } \\
\text { sensitizer }\end{array}$ & & & & & ICCVAM (2013) & \\
\hline $\begin{array}{l}\text { (2-oxo-1-phenyl- } \\
\text { pyrrolidin-3- } \\
\text { yl)(triphenyl)- } \\
\text { phosphonium bromide }\end{array}$ & $\begin{array}{l}\mathrm{O}=\mathrm{C} 1 \mathrm{C}(\mathrm{CCN} 1 \mathrm{c} 1 \mathrm{ccccc} 1) \\
{[\mathrm{P}+](\mathrm{c} 1 \mathrm{ccccc} 1)(\mathrm{c} 1 \mathrm{ccccc}} \\
1) \mathrm{c} 1 \mathrm{ccccc} 1\end{array}$ & & $\begin{array}{l}\text { Non- } \\
\text { sensitizer }\end{array}$ & & & & & ICCVAM (2013) & \\
\hline $\begin{array}{l}\text { (2R)-2-amino-3-[(2R)-2- } \\
\text { amino-3-hydroxy-3- } \\
\text { oxopropyl]disulfanylpro } \\
\text { panoic acid }\end{array}$ & $\begin{array}{l}N C(\operatorname{CsSCC}(N) C(O)=0) C( \\
O)=0\end{array}$ & & $\begin{array}{l}\text { Non- } \\
\text { sensitizer }\end{array}$ & & & & & REACH (2008-2014) & \\
\hline
\end{tabular}




\begin{tabular}{|c|c|c|c|c|c|c|c|c|c|}
\hline Compound name & SMILES & $\begin{array}{l}\text { Human } \\
\text { result }\end{array}$ & $\begin{array}{l}\text { LLNA } \\
\text { result }\end{array}$ & DPRA & KeratinoSens & h-CLAT & $\begin{array}{l}\text { Human } \\
\text { reference }\end{array}$ & LLNA reference & Non-animal reference \\
\hline $\begin{array}{l}\text { (2R,3Z,5R)-3-(2- } \\
\text { hydroxyethylidene)-7- } \\
\text { oxo-4-oxa-1- } \\
\text { azabicyclo[3.2.0]heptan } \\
\text { e-2-carboxylic acid; } 2- \\
\text { methylpropan-2-amine }\end{array}$ & $\begin{array}{l}\mathrm{OCC}=\mathrm{C} 1 \mathrm{OC} 2 \mathrm{CC}(=0) \mathrm{N} 2 \\
\mathrm{C} 1 \mathrm{C}(\mathrm{O})=\mathrm{O}\end{array}$ & & $\begin{array}{l}\text { Non- } \\
\text { sensitizer }\end{array}$ & & & & & REACH (2008-2014) & \\
\hline $\begin{array}{l}\text { (2R,4S)-4-(4-acetyl-1- } \\
\text { piperazinyl)-N-\{(1R)-1- } \\
{[3,5-\text { bis(trifluoro- }} \\
\text { methyl)phenyl]-ethyl\}- } \\
\text { 2-(4-fluoro-2- } \\
\text { methylphenyl)-N- } \\
\text { methyl-1-piperidine- } \\
\text { carboxamide } \\
\text { monomethane- } \\
\text { sulfonate }\end{array}$ & $\begin{array}{l}\mathrm{CC}(\mathrm{N}(\mathrm{C}) \mathrm{C}(=\mathrm{O}) \mathrm{N} 1 \mathrm{CCC}(\mathrm{C} \\
\mathrm{C} 1 \mathrm{c} 1 \mathrm{ccc}(\mathrm{F}) \mathrm{cc} 1 \mathrm{C}) \mathrm{N} 1 \mathrm{CCN} \\
(\mathrm{CC} 1) \mathrm{C}(\mathrm{C})=\mathrm{O}) \mathrm{c} 1 \mathrm{cc}(\mathrm{cc}(\mathrm{c} \\
1) \mathrm{C}(\mathrm{F})(\mathrm{F}) \mathrm{F}) \mathrm{C}(\mathrm{F})(\mathrm{F}) \mathrm{F}\end{array}$ & & $\begin{array}{l}\text { Non- } \\
\text { sensitizer }\end{array}$ & & & & & ICCVAM (2013) & \\
\hline $\begin{array}{l}\text { (2S)-2-hydroxy-3- } \\
\text { (phenylamino)propane- } \\
\text { 1-sulfonate }\end{array}$ & $\begin{array}{l}\mathrm{OC}(\mathrm{CNc} 1 \mathrm{ccccc} 1) \operatorname{CS}(0)( \\
=0)=0\end{array}$ & & $\begin{array}{l}\text { Non- } \\
\text { sensitizer }\end{array}$ & & & & & REACH (2008-2014) & \\
\hline $\begin{array}{l}\text { (2S)-5-amino-2-[(2- } \\
\text { azaniumylacetyl)amino] } \\
-5 \text {-oxopentanoate }\end{array}$ & $\begin{array}{l}N C C(=0) N C(C C C(N)=0) \\
C(O)=0\end{array}$ & & $\begin{array}{l}\text { Non- } \\
\text { sensitizer }\end{array}$ & & & & & REACH (2008-2014) & \\
\hline $\begin{array}{l}\text { (2S)-5-hydroxy-2-(3- } \\
\text { hydroxy-4- } \\
\text { methoxyphenyl)-7- } \\
{[(2 S, 3 R, 4 S, 5 S, 6 R)-3,4,5-} \\
\text { trihydroxy-6- } \\
{[[(2 R, 3 R, 4 R, 5 R, 6 S)-} \\
\text { 3,4,5-trihydroxy-6- } \\
\text { methyloxan-2- } \\
\text { yl]oxymethyl]oxan-2- } \\
\text { yl]oxychroman-4-one }\end{array}$ & $\begin{array}{l}\operatorname{cOc} 1 \mathrm{ccc}(\mathrm{cc} 10) \mathrm{C} 1 \mathrm{CC}(= \\
\mathrm{O}) \mathrm{c} 2 \mathrm{c}(\mathrm{O}) \mathrm{cc}(\mathrm{OC} 3 \mathrm{OC}(\mathrm{cO} \\
\mathrm{C} 4 \mathrm{OC}(\mathrm{C}) \mathrm{C}(\mathrm{O}) \mathrm{C}(0) \mathrm{C} 4 \mathrm{O}) \\
\mathrm{C}(\mathrm{O}) \mathrm{C}(0) \mathrm{O} 3 \mathrm{O}) \mathrm{cc} 2 \mathrm{O} 1\end{array}$ & & $\begin{array}{l}\text { Non- } \\
\text { sensitizer }\end{array}$ & & & & & REACH (2008-2014) & \\
\hline (2S)-hexane-1,2-diol & $\mathrm{CCCCC}(\mathrm{O}) \mathrm{CO}$ & & $\begin{array}{l}\text { Non- } \\
\text { sensitizer }\end{array}$ & & & & & REACH (2008-2014) & \\
\hline $\begin{array}{l}\text { (2S,4S)-1-[(2S)-2-amino- } \\
\text { 3,3-bis(4-fluorophenyl)- } \\
\text { 1-oxopropyl]-4-fluoro- } \\
\text { 2-pyrrolidine } \\
\text { carbonitrile }\end{array}$ & $\begin{array}{l}N C(C(c 1 c c c(F) c c 1) c 1 c c \\
c(F) c c 1) C(=0) N 1 C C(F) C \\
C 1 C \# N\end{array}$ & & $\begin{array}{l}\text { Non- } \\
\text { sensitizer }\end{array}$ & & & & & ICCVAM (2013) & \\
\hline $\begin{array}{l}\text { (2S,5R,6R)-6-amino-3,3- } \\
\text { dimethyl-7-oxo-4-thia- } \\
\text { 1- } \\
\text { azabicyclo[3.2.0]heptan } \\
\text { e-2-carboxylic acid }\end{array}$ & $\begin{array}{l}\mathrm{CC} 1(\mathrm{C}) \mathrm{SC} 2 \mathrm{C}(\mathrm{N}) \mathrm{C}(=\mathrm{O}) \mathrm{N} \\
2 \mathrm{C} 1 \mathrm{C}(\mathrm{O})=\mathrm{O}\end{array}$ & & $\begin{array}{l}\text { Non- } \\
\text { sensitizer }\end{array}$ & & & & & REACH (2008-2014) & \\
\hline
\end{tabular}




\begin{tabular}{|c|c|c|c|c|c|c|c|c|c|}
\hline Compound name & SMILES & $\begin{array}{l}\text { Human } \\
\text { result }\end{array}$ & $\begin{array}{l}\text { LLNA } \\
\text { result }\end{array}$ & DPRA & KeratinoSens & h-CLAT & $\begin{array}{l}\text { Human } \\
\text { reference }\end{array}$ & LLNA reference & Non-animal reference \\
\hline $\begin{array}{l}\text { (3-hydroxy-2,2- } \\
\text { dimethylpropyl) 3- } \\
\text { hydroxy-2,2- } \\
\text { dimethylpropanoate }\end{array}$ & $\begin{array}{l}\mathrm{CC}(\mathrm{C})(\mathrm{CO}) \mathrm{COC}(=\mathrm{O}) \mathrm{C}(\mathrm{C}) \\
(\mathrm{C}) \mathrm{CO}\end{array}$ & & $\begin{array}{l}\text { Non- } \\
\text { sensitizer }\end{array}$ & & & & & REACH (2008-2014) & \\
\hline $\begin{array}{l}\text { (3E)-3-[(4-chloro-2- } \\
\text { nitrophenyl)hydrazinyli } \\
\text { dene]-2- } \\
\text { methylpyrazolo[5,1- } \\
\text { b]quinazolin-9-one }\end{array}$ & $\begin{array}{l}\text { Cc1nn2c(Nc3cccce3C2 } \\
=0) c 1 N=N c 1 \operatorname{ccc}(\mathrm{Cl}) \mathrm{cc} 1 \\
\mathrm{~N}(=\mathrm{O})=\mathrm{O}\end{array}$ & & $\begin{array}{l}\text { Non- } \\
\text { sensitizer }\end{array}$ & & & & & REACH (2008-2014) & \\
\hline $\begin{array}{l}(3 R, 3 a S, 6 a R)- \\
\text { hexahydrofuro-[2,3- } \\
\text { b]furan-3-yl [(1S,2R)-3- } \\
\text { [(1,3-benzodioxol-5- } \\
\text { ylsulfonyl)(2- } \\
\text { methylpropyl)-amino]- } \\
\text { 2-hydroxy-1-[[4-[(2- } \\
\text { methyl-4- } \\
\text { thiazolyl)methoxy]phen } \\
\text { yl]methyl]- } \\
\text { propyl]carbamate }\end{array}$ & $\begin{array}{l}\mathrm{CC}(\mathrm{C}) \mathrm{CN}(\mathrm{CC}(\mathrm{O}) \mathrm{C}(\mathrm{Cc} 1 \mathrm{cc} \\
\mathrm{c}(\mathrm{OCc} 2 \mathrm{csc}(\mathrm{C}) \mathrm{n} 2) \mathrm{cc} 1) \mathrm{NC} \\
(=0) \mathrm{OC} 1 \mathrm{COC} 2 \mathrm{OCCC12}) \\
\mathrm{S}(=0)(=0) \mathrm{c} 1 \mathrm{ccc} 2 \mathrm{OCOc} \\
2 \mathrm{c} 1\end{array}$ & & $\begin{array}{l}\text { Non- } \\
\text { sensitizer }\end{array}$ & & & & & ICCVAM (2013) & \\
\hline $\begin{array}{l}\text { (3R,3aS,6aR)- } \\
\text { hexahydrofuro[2,3- } \\
\text { b]furan-3-ol }\end{array}$ & OC1COC2OCCC12 & & $\begin{array}{l}\text { Non- } \\
\text { sensitizer }\end{array}$ & & & & & ICCVAM (2013) & \\
\hline $\begin{array}{l}\text { (3R,6Rr)-3-(2,3-dihydro- } \\
1 \mathrm{H} \text {-inden-2-yl)-1-[(1R)- } \\
\text { 1-(2-methyl-1,3-oxazol- } \\
4 \text {-yl)-2-(4-morpholinyl)- } \\
\text { 2-oxoethyl]-6-[(1S)-1- } \\
\text { methylpropyl]-2,5- } \\
\text { piperazinedione }\end{array}$ & $\begin{array}{l}\mathrm{CCC}(\mathrm{C}) \mathrm{C} 1 \mathrm{~N}(\mathrm{C}(\mathrm{C}(=\mathrm{O}) \mathrm{N} 2 \\
\mathrm{CCOCC} 2) \mathrm{c} 2 \operatorname{coc}(\mathrm{C}) \mathrm{n} 2) \mathrm{C}( \\
=0) \mathrm{C}(\mathrm{NC} 1=0) \mathrm{C} 1 \mathrm{Cc} 2 \mathrm{ccc} \\
\mathrm{cc} 2 \mathrm{C} 1\end{array}$ & & $\begin{array}{l}\text { Non- } \\
\text { sensitizer }\end{array}$ & & & & & ICCVAM (2013) & \\
\hline $\begin{array}{l}\text { (3S,6R)-3-isopropyl-6- } \\
\text { methylcyclohexene }\end{array}$ & $\mathrm{CC}(\mathrm{C}) \mathrm{C} 1 \mathrm{CCC}(\mathrm{C}) \mathrm{C}=\mathrm{C} 1$ & & $\begin{array}{l}\text { Non- } \\
\text { sensitizer }\end{array}$ & & & & & ICCVAM (2013) & \\
\hline $\begin{array}{l}\text { (4E)-4-[(2,5- } \\
\text { dichlorophenyl)hydrazi } \\
\text { nylidene]-3-oxo-N- } \\
\text { phenylnaphthalene-2- } \\
\text { carboxamide }\end{array}$ & $\begin{array}{l}\text { Oc1c(cc2ccccc2c1N=N } \\
\operatorname{c1cc}(\mathrm{Cl}) \operatorname{ccc} 1 \mathrm{Cl}) \mathrm{C}(=\mathrm{O}) \mathrm{Nc} \\
1 \mathrm{ccccc} 1\end{array}$ & & $\begin{array}{l}\text { Non- } \\
\text { sensitizer }\end{array}$ & & & & & REACH (2008-2014) & \\
\hline $\begin{array}{l}\text { (4E)-4-[(4- } \\
\text { carbamoylphenyl)hydra } \\
\text { zinylidene]-N-(2- } \\
\text { ethoxyphenyl)-3- } \\
\text { oxonaphthalene-2- } \\
\text { carboxamide }\end{array}$ & $\begin{array}{l}\mathrm{CCOc} 1 \mathrm{ccccc} 1 \mathrm{NC}(=\mathrm{O}) \mathrm{c} 1 \\
\operatorname{cc} 2 \operatorname{ccccc} 2 \mathrm{c}(\mathrm{N}=\mathrm{Nc} 2 \mathrm{ccc}( \\
\mathrm{cc} 2) \mathrm{C}(\mathrm{N})=\mathrm{O}) \mathrm{c} 10\end{array}$ & & $\begin{array}{l}\text { Non- } \\
\text { sensitizer }\end{array}$ & & & & & REACH (2008-2014) & \\
\hline
\end{tabular}




\begin{tabular}{|c|c|c|c|c|c|c|c|c|c|}
\hline Compound name & SMILES & $\begin{array}{l}\text { Human } \\
\text { result }\end{array}$ & $\begin{array}{l}\text { LLNA } \\
\text { result }\end{array}$ & DPRA & KeratinoSens & h-CLAT & $\begin{array}{l}\text { Human } \\
\text { reference }\end{array}$ & LLNA reference & Non-animal reference \\
\hline $\begin{array}{l}\text { (4E)-4-[[2-chloro-4-[3- } \\
\text { chloro-4-[(2E)-2-(3- } \\
\text { methyl-5-oxo-1- } \\
\text { phenylpyrazol-4- } \\
\text { ylidene)hydrazinyl]phen } \\
\text { yl]phenyl]hydrazinylide } \\
\text { ne]-5-methyl-2- } \\
\text { phenylpyrazol-3-one }\end{array}$ & $\begin{array}{l}\mathrm{CC} 1=\mathrm{NN}(\mathrm{C}(=\mathrm{O}) \mathrm{C} 1=\mathrm{NNc} \\
1 \mathrm{ccc}(\mathrm{cc} 1 \mathrm{Cl})- \\
\mathrm{c} 1 \mathrm{ccc}(\mathrm{NN}=\mathrm{C} 2 \mathrm{C}(\mathrm{C})=\mathrm{NN}( \\
\mathrm{C} 2=\mathrm{O}) \mathrm{c} 2 \mathrm{ccccc} 2) \mathrm{c}(\mathrm{Cl}) \mathrm{c} 1) \\
\mathrm{c} 1 \mathrm{ccccc} 1\end{array}$ & & $\begin{array}{l}\text { Non- } \\
\text { sensitizer }\end{array}$ & & & & & REACH (2008-2014) & \\
\hline $\begin{array}{l}\text { (4E)-4-[[2-chloro-5- } \\
\text { (trifluoromethyl)phenyl } \\
\text { ]hydrazinylidene]-N- } \\
\text { [2,5-dichloro-4-[[(4Z)-4- } \\
\text { [[2-chloro-5- } \\
\text { (trifluoromethyl)phenyl } \\
\text { ]hydrazinylidene]-3- } \\
\text { oxonaphthalene-2- } \\
\text { carbonyl]amino]phenyl] } \\
\text {-3-oxonaphthalene-2- } \\
\text { carboxamide }\end{array}$ & $\begin{array}{l}\text { Oc1c(cc2ccccc2c1N=N } \\
\operatorname{c1cc}(\operatorname{ccc} 1 \mathrm{Cl}) \mathrm{C}(\mathrm{F})(\mathrm{F}) \mathrm{F}) \mathrm{Cl} \\
=\mathrm{O}) \mathrm{Nc} 1 \mathrm{cc}(\mathrm{Cl}) \mathrm{c}(\mathrm{NC}(=\mathrm{O}) \mathrm{c} \\
2 \mathrm{cc} 3 \operatorname{ccccc} 3 \mathrm{c}(\mathrm{N}=\mathrm{Nc3} \operatorname{cc}( \\
\operatorname{ccc} 3 \mathrm{Cl}) \mathrm{C}(\mathrm{F})(\mathrm{F}) \mathrm{F}) \mathrm{c} 2 \mathrm{O}) \mathrm{cc} \\
1 \mathrm{Cl}\end{array}$ & & $\begin{array}{l}\text { Non- } \\
\text { sensitizer }\end{array}$ & & & & & REACH (2008-2014) & \\
\hline $\begin{array}{l}\text { (4E)-4-[[5-[(4- } \\
\text { carbamoylphenyl)carba } \\
\text { moyl]-2- } \\
\text { methoxyphenyl]hydrazi } \\
\text { nylidene]-N-(5-chloro- } \\
\text { 2,4-dimethoxyphenyl)- } \\
\text { 3-oxonaphthalene-2- } \\
\text { carboxamide }\end{array}$ & $\begin{array}{l}\operatorname{cOc} 1 \mathrm{cc}(\mathrm{OC}) \mathrm{c}(\mathrm{NC}(=\mathrm{O}) \mathrm{c2} \\
\operatorname{cc} 3 \mathrm{ccccc} 3 \mathrm{c}(\mathrm{N}=\mathrm{Nc3} \operatorname{cc}(\mathrm{c} \\
\mathrm{cc} 3 \mathrm{OC}) \mathrm{C}(=\mathrm{O}) \mathrm{Nc} 3 \mathrm{ccc}(\mathrm{cc} \\
3) \mathrm{C}(\mathrm{N})=\mathrm{O}) \mathrm{c} 2 \mathrm{O}) \mathrm{cc} 1 \mathrm{Cl}\end{array}$ & & $\begin{array}{l}\text { Non- } \\
\text { sensitizer }\end{array}$ & & & & & REACH (2008-2014) & \\
\hline $\begin{array}{l}\text { (4E)-N-[2,5-dichloro-4- } \\
\text { [[(4Z)-4-[(2,5- } \\
\text { dichlorophenyl)hydrazi } \\
\text { nylidene]-3- } \\
\text { oxonaphthalene-2- } \\
\text { carbonyl]amino]phenyl] } \\
-4-[(2,5- \\
\text { dichlorophenyl)hydrazi } \\
\text { nylidene]-3- } \\
\text { oxonaphthalene-2- } \\
\text { carboxamide }\end{array}$ & $\begin{array}{l}\text { Oc1c(cc2ccccc2c1N=N } \\
\operatorname{c1cc}(\mathrm{Cl}) \operatorname{ccc} 1 \mathrm{Cl}) \mathrm{C}(=\mathrm{O}) \mathrm{Nc} \\
1 \mathrm{cc}(\mathrm{Cl}) \mathrm{c}(\mathrm{NC}(=\mathrm{O}) \mathrm{c} 2 \mathrm{cc} 3 \mathrm{c} \\
\operatorname{cccc} 3 \mathrm{c}(\mathrm{N}=\mathrm{Nc} 3 \mathrm{cc}(\mathrm{Cl}) \mathrm{ccc} \\
3 \mathrm{Cl}) \mathrm{c} 2 \mathrm{O}) \mathrm{cc} 1 \mathrm{Cl}\end{array}$ & & $\begin{array}{l}\text { Non- } \\
\text { sensitizer }\end{array}$ & & & & & REACH (2008-2014) & \\
\hline
\end{tabular}




\begin{tabular}{|c|c|c|c|c|c|c|c|c|c|}
\hline Compound name & SMILES & $\begin{array}{l}\text { Human } \\
\text { result }\end{array}$ & $\begin{array}{l}\text { LLNA } \\
\text { result }\end{array}$ & DPRA & KeratinoSens & h-CLAT & $\begin{array}{l}\text { Human } \\
\text { reference }\end{array}$ & LLNA reference & Non-animal reference \\
\hline $\begin{array}{l}\text { (4E)-N-[2-chloro-4- } \\
\text { [[(4E)-4-[(2,5- } \\
\text { dichlorophenyl)hydrazi } \\
\text { nylidene]-3- } \\
\text { oxonaphthalene-2- } \\
\text { carbonyl]amino]phenyl] } \\
-4-[(2,5- \\
\text { dichlorophenyl)hydrazi } \\
\text { nylidene]-3- } \\
\text { oxonaphthalene-2- } \\
\text { carboxamide }\end{array}$ & $\begin{array}{l}\text { Oc1c(cc2ccccc2 } 1 \mathrm{~N}=\mathrm{N} \\
\mathrm{c1} 1 \mathrm{cc}(\mathrm{Cl}) \mathrm{ccc} 1 \mathrm{Cl}) \mathrm{C}(=\mathrm{O}) \mathrm{Nc} \\
1 \mathrm{ccc}(\mathrm{NC}(=\mathrm{O}) \mathrm{c} 2 \mathrm{cc} 3 \mathrm{cccc} \\
\mathrm{c} 3 \mathrm{c}(\mathrm{N}=\mathrm{Nc} 3 \mathrm{cc}(\mathrm{Cl}) \mathrm{ccc} 3 \mathrm{Cl} \\
) \mathrm{c} 2 \mathrm{O}) \mathrm{c}(\mathrm{Cl}) \mathrm{c} 1\end{array}$ & & $\begin{array}{l}\text { Non- } \\
\text { sensitizer }\end{array}$ & & & & & REACH (2008-2014) & \\
\hline $\begin{array}{l}\text { (4R,5S)-(-)-1,5- } \\
\text { Dimethyl-4-phenyl-2- } \\
\text { imidazolidinone }\end{array}$ & $\begin{array}{l}\operatorname{cC1C}(\mathrm{NC}(=0) \mathrm{N} 1 \mathrm{C}) \mathrm{c} 1 \mathrm{cc} \\
\operatorname{ccc} 1\end{array}$ & & $\begin{array}{l}\text { Non- } \\
\text { sensitizer }\end{array}$ & & & & & ICCVAM (2013) & \\
\hline $\begin{array}{l}\text { (4S)-1-(tert- } \\
\text { butoxycarbonyl)-4- } \\
\text { fluoro-L-proline }\end{array}$ & $\begin{array}{l}\mathrm{CC}(\mathrm{C})(\mathrm{C}) \mathrm{OC}(=0) \mathrm{N} 1 \mathrm{CC}(\mathrm{F} \\
\operatorname{CC} 1 \mathrm{C}(\mathrm{O})=\mathrm{O}\end{array}$ & & $\begin{array}{l}\text { Non- } \\
\text { sensitizer }\end{array}$ & & & & & ICCVAM (2013) & \\
\hline $\begin{array}{l}\text { (4S,5R)-1-[(1R,2R,3S)-3- } \\
\text { (1,3-Benzodioxol-5-yl)- } \\
\text { 1-(2-benzyloxy-4- } \\
\text { methoxyphenyl)-1- } \\
\text { hydroxy-6-propoxy-2- } \\
\text { indanoyl]-3,4-dimethyl- } \\
\text { 5-phenyl-2- } \\
\text { imidazolidinone }\end{array}$ & $\begin{array}{l}\mathrm{CCCOc1} 1 \mathrm{ccc} 2 \mathrm{C}(\mathrm{C}(\mathrm{C}(=)) \\
\mathrm{N} 3 \mathrm{C}(\mathrm{C}(\mathrm{C}) \mathrm{N}(\mathrm{C}) \mathrm{C} 3=0) \mathrm{c} 3 \mathrm{c} \\
\mathrm{cccc} 3) \mathrm{C}(\mathrm{O})(\mathrm{c} 2 \mathrm{c} 1) \mathrm{c} 1 \mathrm{ccc}( \\
\mathrm{OC}) \mathrm{cc} 1 \mathrm{OCc} 1 \mathrm{ccccc} 1) \mathrm{c} 1 \mathrm{c} \\
\mathrm{cc} 2 \mathrm{OCO} 2 \mathrm{c} 1\end{array}$ & & $\begin{array}{l}\text { Non- } \\
\text { sensitizer }\end{array}$ & & & & & ICCVAM (2013) & \\
\hline $\begin{array}{l}\text { (4Z)-2-Methyl-6- } \\
\text { methyleneoct-4-ene }\end{array}$ & $\mathrm{CCC}(=\mathrm{C}) \mathrm{C}=\mathrm{CCC}(\mathrm{C}) \mathrm{C}$ & & $\begin{array}{l}\text { Non- } \\
\text { sensitizer }\end{array}$ & & & & & ICCVAM (2013) & \\
\hline $\begin{array}{l}\text { (4Z)-4-[(2-methyl-4- } \\
\text { nitrophenyl)hydrazinyli } \\
\text { dene]-N-(2- } \\
\text { methylphenyl)-3- } \\
\text { oxonaphthalene-2- } \\
\text { carboxamide }\end{array}$ & $\begin{array}{l}\mathrm{Cc} 1 \mathrm{ccccc} 1 \mathrm{NC}(=\mathrm{O}) \mathrm{c} 1 \mathrm{cc} 2 \\
\operatorname{ccccc} 2 \mathrm{c}(\mathrm{N}=\mathrm{Nc} 2 \operatorname{ccc}(\mathrm{ccc} 2 \\
\mathrm{C}) \mathrm{N}(=\mathrm{O})=\mathrm{O}) \mathrm{c} 1 \mathrm{O}\end{array}$ & & $\begin{array}{l}\text { Non- } \\
\text { sensitizer }\end{array}$ & & & & & REACH (2008-2014) & \\
\hline $\begin{array}{l}\text { (4Z)-4-[[2,5-dimethoxy- } \\
4- \\
\text { (methylsulfamoyl)phen } \\
\text { yl]hydrazinylidene]-3- } \\
\text { oxo-N-(2-oxo-1,3- } \\
\text { dihydrobenzimidazol-5- } \\
\text { yl)naphthalene-2- } \\
\text { carboxamide }\end{array}$ & $\begin{array}{l}\mathrm{CNS}(=\mathrm{O})(=\mathrm{O}) \mathrm{c} 1 \mathrm{cc}(\mathrm{OC}) \mathrm{c} \\
(\mathrm{cc1OC}) \mathrm{N}=\mathrm{Nc1}(\mathrm{O}) \mathrm{c}(\mathrm{cc} \\
2 \mathrm{ccccc12}) \mathrm{C}(=\mathrm{O}) \mathrm{Nc} 1 \mathrm{ccc} \\
2 \mathrm{NC}(=0) \mathrm{Nc} 2 \mathrm{c} 1\end{array}$ & & $\begin{array}{l}\text { Non- } \\
\text { sensitizer }\end{array}$ & & & & & REACH (2008-2014) & \\
\hline
\end{tabular}




\begin{tabular}{|c|c|c|c|c|c|c|c|c|c|}
\hline Compound name & SMILES & $\begin{array}{l}\text { Human } \\
\text { result }\end{array}$ & $\begin{array}{l}\text { LLNA } \\
\text { result }\end{array}$ & DPRA & KeratinoSens & h-CLAT & $\begin{array}{l}\text { Human } \\
\text { reference }\end{array}$ & LLNA reference & Non-animal reference \\
\hline $\begin{array}{l}\text { (4Z)-4-[[2-methoxy-5- } \\
\text { (phenylcarbamoyl)phen } \\
\text { yl]hydrazinylidene]-3- } \\
\text { oxo-N-(2-oxo-1,3- } \\
\text { dihydrobenzimidazol-5- } \\
\text { yl)naphthalene-2- } \\
\text { carboxamide }\end{array}$ & $\begin{array}{l}\operatorname{COc} 1 \mathrm{ccc}(\operatorname{cc} 1 \mathrm{~N}=\mathrm{Nc} 1 \mathrm{c}(\mathrm{O} \\
) \mathrm{c}(\mathrm{cc} 2 \operatorname{ccccc} 12) \mathrm{C}(=\mathrm{O}) \mathrm{Nc} \\
1 \mathrm{ccc} 2 \mathrm{NC}(=\mathrm{O}) \mathrm{Nc} 2 \mathrm{c} 1) \mathrm{C}(= \\
\mathrm{O}) \mathrm{Nc} 1 \mathrm{ccccc} 1\end{array}$ & & $\begin{array}{l}\text { Non- } \\
\text { sensitizer }\end{array}$ & & & & & REACH (2008-2014) & \\
\hline $\begin{array}{l}\text { (4Z)-4-[[2-methoxy-5- } \\
\text { methyl-4- } \\
\text { (methylsulfamoyl)phen } \\
\text { yl]hydrazinylidene]-3- } \\
\text { oxo-N-(2-oxo-1,3- } \\
\text { dihydrobenzimidazol-5- } \\
\text { yl)naphthalene-2- } \\
\text { carboxamide }\end{array}$ & $\begin{array}{l}\mathrm{CNS}(=\mathrm{O})(=\mathrm{O}) \mathrm{c} 1 \mathrm{cc}(\mathrm{OC}) \mathrm{c} \\
(\operatorname{cc1C}) \mathrm{N}=\mathrm{Nc1c}(\mathrm{O}) \mathrm{c}(\mathrm{cc} 2 \\
\operatorname{ccccc12} \mathrm{C}(=\mathrm{O}) \mathrm{Nc} 1 \mathrm{ccc} 2 \\
\mathrm{NC}(=\mathrm{O}) \mathrm{Nc} 2 \mathrm{c1}\end{array}$ & & $\begin{array}{l}\text { Non- } \\
\text { sensitizer }\end{array}$ & & & & & REACH (2008-2014) & \\
\hline $\begin{array}{l}\text { (4Z)-N-(4- } \\
\text { acetamidophenyl)-4- } \\
\text { [(5-carbamoyl-2- } \\
\text { chlorophenyl)hydrazinyl } \\
\text { idene]-3- } \\
\text { oxonaphthalene-2- } \\
\text { carboxamide }\end{array}$ & $\begin{array}{l}\mathrm{CC}(=0) \mathrm{Nc} 1 \mathrm{ccc}(\mathrm{NC}(=\mathrm{O}) \mathrm{c} \\
2 \operatorname{cc} 3 \operatorname{ccccc} 3 \mathrm{c}(\mathrm{N}=\mathrm{Nc} 3 \mathrm{cc}( \\
\operatorname{ccc} 3 \mathrm{Cl}) \mathrm{C}(\mathrm{N})=\mathrm{O}) \mathrm{c} 2 \mathrm{O}) \mathrm{cc} 1\end{array}$ & & $\begin{array}{l}\text { Non- } \\
\text { sensitizer }\end{array}$ & & & & & REACH (2008-2014) & \\
\hline $\begin{array}{l}\text { (4Z)-N-(4-chloro-2,5- } \\
\text { dimethoxyphenyl)-4- } \\
\text { [[2-methoxy-5- } \\
\text { (phenylcarbamoyl)phen } \\
\text { yl]hydrazinylidene]-3- } \\
\text { oxonaphthalene-2- } \\
\text { carboxamide }\end{array}$ & $\begin{array}{l}\operatorname{cOc} 1 \mathrm{cc}(\mathrm{NC}(=\mathrm{O}) \mathrm{c} 2 \mathrm{cc} 3 \mathrm{cc} \\
\mathrm{ccc} 3 \mathrm{c}(\mathrm{N}=\mathrm{Nc} 3 \mathrm{cc}(\mathrm{ccc} 3 \mathrm{OC} \\
\mathrm{C}(=\mathrm{O}) \mathrm{Nc} 3 \mathrm{ccccc} 3) \mathrm{c} 2 \mathrm{O}) \\
\mathrm{c}(\mathrm{OC}) \mathrm{cc} 1 \mathrm{Cl}\end{array}$ & & $\begin{array}{l}\text { Non- } \\
\text { sensitizer }\end{array}$ & & & & & REACH (2008-2014) & \\
\hline $\begin{array}{l}\text { (4Z)-N-(5-chloro-2- } \\
\text { methoxyphenyl)-4-[[2- } \\
\text { methoxy-5- } \\
\text { (phenylcarbamoyl)phen } \\
\text { yl]hydrazinylidene]-3- } \\
\text { oxonaphthalene-2- } \\
\text { carboxamide }\end{array}$ & $\begin{array}{l}\operatorname{cOc} 1 \mathrm{ccc}(\mathrm{Cl}) \operatorname{cc} 1 \mathrm{NC}(=\mathrm{O}) \\
\mathrm{c} 1 \mathrm{cc} 2 \mathrm{ccccc} 2 \mathrm{c}(\mathrm{N}=\mathrm{Nc} 2 \mathrm{cc} \\
(\mathrm{ccc} 2 \mathrm{OC}) \mathrm{C}(=\mathrm{O}) \mathrm{Nc} 2 \mathrm{cccc} \\
\mathrm{c} 2) \mathrm{c} 10\end{array}$ & & $\begin{array}{l}\text { Non- } \\
\text { sensitizer }\end{array}$ & & & & & REACH (2008-2014) & \\
\hline $\begin{array}{l}\text { (4Z)-N-(5-chloro-2- } \\
\text { methylphenyl)-4-[[2- } \\
\text { methoxy-5- } \\
\text { (phenylcarbamoyl)phen } \\
\text { yl]hydrazinylidene]-3- } \\
\text { oxonaphthalene-2- } \\
\text { carboxamide }\end{array}$ & $\begin{array}{l}\operatorname{cOc} 1 \operatorname{ccc}(\operatorname{cc} 1 \mathrm{~N}=\mathrm{Nc} 1 \mathrm{c}(\mathrm{O} \\
) \mathrm{c}(\mathrm{cc} 2 \operatorname{ccccc} 12) \mathrm{C}(=\mathrm{O}) \mathrm{Nc} \\
1 \mathrm{cc}(\mathrm{Cl}) \operatorname{ccc} 1 \mathrm{C}) \mathrm{C}(=\mathrm{O}) \mathrm{Nc1} \\
\operatorname{ccccc} 1\end{array}$ & & $\begin{array}{l}\text { Non- } \\
\text { sensitizer }\end{array}$ & & & & & REACH (2008-2014) & \\
\hline
\end{tabular}




\begin{tabular}{|c|c|c|c|c|c|c|c|c|c|}
\hline Compound name & SMILES & $\begin{array}{l}\text { Human } \\
\text { result }\end{array}$ & $\begin{array}{l}\text { LLNA } \\
\text { result }\end{array}$ & DPRA & KeratinoSens & h-CLAT & $\begin{array}{l}\text { Human } \\
\text { reference }\end{array}$ & LLNA reference & Non-animal reference \\
\hline $\begin{array}{l}\text { (5-amino-3-hydroxy-5- } \\
\text { oxopentylidene)- } \\
\text { dimethylazanium } \\
\text { chloride }\end{array}$ & $\begin{array}{l}\mathrm{C}[\mathrm{N}+](\mathrm{C})(\mathrm{C}) \mathrm{CC}(\mathrm{O}) \mathrm{CC}(\mathrm{N}) \\
=0\end{array}$ & & $\begin{array}{l}\text { Non- } \\
\text { sensitizer }\end{array}$ & & & & & REACH (2008-2014) & \\
\hline $\begin{array}{l}\text { (5-ethyl-1,3-dioxan-5- } \\
\text { yl)methanol }\end{array}$ & $\mathrm{CCC} 1(\mathrm{CO}) \mathrm{COCOC1}$ & & $\begin{array}{l}\text { Non- } \\
\text { sensitizer }\end{array}$ & & & & & REACH (2008-2014) & \\
\hline $\begin{array}{l}\text { (6-methyl-2-oxo-1,3- } \\
\text { diazinan-4-yl)urea }\end{array}$ & $\begin{array}{l}\mathrm{CC1CC}(\mathrm{NC}(\mathrm{N})=\mathrm{O}) \mathrm{NC}(= \\
\mathrm{O}) \mathrm{N} 1\end{array}$ & & $\begin{array}{l}\text { Non- } \\
\text { sensitizer }\end{array}$ & & & & & REACH (2008-2014) & \\
\hline $\begin{array}{l}\text { (8S,9S,10R,11S,13S,14S, } \\
\text { 17R)-11,17-dihydroxy- } \\
\text { 17-(2-hydroxyacetyl)- } \\
\text { 10,13-dimethyl- } \\
\text { 7,8,9,11,12,14,15,16- } \\
\text { octahydro-6H- } \\
\text { cyclopenta[a]phenanthr } \\
\text { en-3-one }\end{array}$ & $\begin{array}{l}\mathrm{CC} 12 \mathrm{CC}(\mathrm{O}) \mathrm{C} 3 \mathrm{C}(\mathrm{CCC} 4= \\
\mathrm{CC}(=\mathrm{O}) \mathrm{C}=\mathrm{CC} 34 \mathrm{C}) \mathrm{C} 1 \mathrm{CC} \\
\mathrm{C} 2(\mathrm{O}) \mathrm{C}(=0) \mathrm{CO}\end{array}$ & & $\begin{array}{l}\text { Non- } \\
\text { sensitizer }\end{array}$ & & & & & REACH (2008-2014) & \\
\hline $\begin{array}{l}\text { (NE)-N-butan-2- } \\
\text { ylidenehydroxylamine }\end{array}$ & $\mathrm{CCC}(\mathrm{C})=\mathrm{NO}$ & & $\begin{array}{l}\text { Non- } \\
\text { sensitizer }\end{array}$ & & & & & REACH (2008-2014) & \\
\hline $\begin{array}{l}\text { (NZ)-N-pentan-2- } \\
\text { ylidenehydroxylamine }\end{array}$ & $\mathrm{CCCC}(\mathrm{C})=\mathrm{NO}$ & & $\begin{array}{l}\text { Non- } \\
\text { sensitizer }\end{array}$ & & & & & REACH (2008-2014) & \\
\hline $\begin{array}{l}\text { (R)-N-Methyl-1-[3,5- } \\
\text { bis(trifluoromethyl)phe } \\
\text { nyl]ethylamine }\end{array}$ & $\begin{array}{l}\text { CNC(C)c1cc(cc(c1)C(F)( } \\
\text { F)F)C(F)(F)F }\end{array}$ & & $\begin{array}{l}\text { Non- } \\
\text { sensitizer }\end{array}$ & & & & & ICCVAM (2013) & \\
\hline $\begin{array}{l}\text { (R,S)-3-amino-2,3,4,5- } \\
\text { tetrahydro-N-(1- } \\
\text { methylethyl)-2,4-dioxo- } \\
\mathrm{N}, 5-\text { diphenyl-1H-1,5- } \\
\text { benzodiazepine-1- } \\
\text { acetamide }\end{array}$ & $\begin{array}{l}\mathrm{CC}(\mathrm{C}) \mathrm{N}(\mathrm{C}(=0) \mathrm{CN} 1 \mathrm{c} 2 \mathrm{ccc} \\
\mathrm{cc} 2 \mathrm{~N}(\mathrm{c} 2 \mathrm{ccccc} 2) \mathrm{C}(=0) \mathrm{C}( \\
\mathrm{N}) \mathrm{C} 1=0) \mathrm{c} 1 \mathrm{ccccc} 1\end{array}$ & & $\begin{array}{l}\text { Non- } \\
\text { sensitizer }\end{array}$ & & & & & ICCVAM (2013) & \\
\hline $\begin{array}{l}\text { (S)-(-)-1-phenylpropyl- } \\
\text { amine }\end{array}$ & $\mathrm{CCC}(\mathrm{N}) \mathrm{c} 1 \mathrm{ccccc} 1$ & & $\begin{array}{l}\text { Non- } \\
\text { sensitizer }\end{array}$ & & & & & ICCVAM (2013) & \\
\hline $\begin{array}{l}\text { (S)-2-hydroxy-2- } \\
\text { methylsuccinic acid }\end{array}$ & $\mathrm{CC}(0)(\mathrm{CC}(0)=0) \mathrm{C}(0)=0$ & & $\begin{array}{l}\text { Non- } \\
\text { sensitizer }\end{array}$ & & & & & ICCVAM (2013) & \\
\hline $\begin{array}{l}\text { (Z)-3-methyl-5-(2,2,3- } \\
\text { trimethyl-1-cyclopent- } \\
\text { 3-enyl)pent-4-en-2-ol }\end{array}$ & $\begin{array}{l}\mathrm{CC}(\mathrm{O}) \mathrm{C}(\mathrm{C}) \mathrm{C}=\mathrm{CC} 1 \mathrm{CC}=\mathrm{C}( \\
\mathrm{C}) \mathrm{C} 1(\mathrm{C}) \mathrm{C}\end{array}$ & & $\begin{array}{l}\text { Non- } \\
\text { sensitizer }\end{array}$ & & & & & REACH (2008-2014) & \\
\hline (Z)-Docos-13-enamide & $\begin{array}{l}\operatorname{CCCCCCCCC}=\operatorname{CCCCCCC} \\
\operatorname{CCCCCC}(N)=0\end{array}$ & & $\begin{array}{l}\text { Non- } \\
\text { sensitizer }\end{array}$ & & & & & REACH (2008-2014) & \\
\hline (Z)-Hex-3-en-1-ol & $\mathrm{CCC}=\mathrm{CCCO}$ & & $\begin{array}{l}\text { Non- } \\
\text { sensitizer }\end{array}$ & & & & & REACH (2008-2014) & \\
\hline $\begin{array}{l}\text { (Z)-octadec-9-en-1-ol; } \\
\text { phosphoric acid }\end{array}$ & $\begin{array}{l}\mathrm{CCCCCCCCC}=\mathrm{CCCCCCC} \\
\mathrm{cCO}\end{array}$ & & $\begin{array}{l}\text { Non- } \\
\text { sensitizer }\end{array}$ & & & & & REACH (2008-2014) & \\
\hline
\end{tabular}




\begin{tabular}{|c|c|c|c|c|c|c|c|c|c|}
\hline Compound name & SMILES & $\begin{array}{l}\text { Human } \\
\text { result }\end{array}$ & $\begin{array}{l}\text { LLNA } \\
\text { result }\end{array}$ & DPRA & KeratinoSens & h-CLAT & $\begin{array}{l}\text { Human } \\
\text { reference }\end{array}$ & LLNA reference & Non-animal reference \\
\hline $\begin{array}{l}\text { (methyl- } \\
\text { phenylcarbamothioyl)s } \\
\text { ulfanyl (methyl- } \\
\text { phenylamino)methaned } \\
\text { ithioate }\end{array}$ & $\begin{array}{l}\mathrm{CN}(\mathrm{C}(=\mathrm{S}) \operatorname{SSC}(=\mathrm{S}) \mathrm{N}(\mathrm{C}) \mathrm{c} 1 \\
\operatorname{ccccc} 1) \operatorname{cocccc} 1\end{array}$ & & $\begin{array}{l}\text { Non- } \\
\text { sensitizer }\end{array}$ & & & & & REACH (2008-2014) & \\
\hline $\begin{array}{l}\text { 1,1,1,2,2,3,3,4,4,5,5,6,6 } \\
\text {-tridecafluorooctane }\end{array}$ & $\begin{array}{l}\mathrm{CCC}(\mathrm{F})(\mathrm{F}) \mathrm{C}(\mathrm{F})(\mathrm{F}) \mathrm{C}(\mathrm{F})(\mathrm{F}) \\
\mathrm{C}(\mathrm{F})(\mathrm{F}) \mathrm{C}(\mathrm{F})(\mathrm{F}) \mathrm{C}(\mathrm{F})(\mathrm{F}) \mathrm{F}\end{array}$ & & $\begin{array}{l}\text { Non- } \\
\text { sensitizer }\end{array}$ & & & & & REACH (2008-2014) & \\
\hline $\begin{array}{l}\text { 1,1,1,3,5,5,5- } \\
\text { Heptamethyl-3- } \\
\text { tetradecyltrisiloxane }\end{array}$ & $\begin{array}{l}\text { CCCCCCCCCCCCCCC[Si]( } \\
\mathrm{C})(\mathrm{O}[\mathrm{Si}](\mathrm{C})(\mathrm{C}) \mathrm{C}) \mathrm{O}[\mathrm{Si}](\mathrm{C}) \\
\text { (C)C }\end{array}$ & & $\begin{array}{l}\text { Non- } \\
\text { sensitizer }\end{array}$ & & & & & REACH (2008-2014) & \\
\hline $\begin{array}{l}1,1,2,2,3,3,4,4,5,5,6,6,7 \\
, 7,8,8,8- \\
\text { heptadecafluorooctane } \\
\text {-1-sulfonate; } \\
\text { tetraethylazanium }\end{array}$ & $\mathrm{CC}[\mathrm{N}+](\mathrm{CC})(\mathrm{CC}) \mathrm{CC}$ & & $\begin{array}{l}\text { Non- } \\
\text { sensitizer }\end{array}$ & & & & & REACH (2008-2014) & \\
\hline $\begin{array}{l}\text { 1,1-Dimethylethyl [(1S)- } \\
\text { 1-[bis(4-fluorophenyl)- } \\
\text { methyl]-2-[(2S,4S)-2- } \\
\text { cyano-4-fluoro-1- } \\
\text { pyrrolidinyl]-2- } \\
\text { oxoethyl]carbamate }\end{array}$ & $\begin{array}{l}\mathrm{CC}(\mathrm{C})(\mathrm{C}) \mathrm{OC}(=0) \mathrm{NC}(\mathrm{C}(\mathrm{c} \\
1 \mathrm{ccc}(\mathrm{F}) \mathrm{cc} 1) \mathrm{c} 1 \mathrm{ccc}(\mathrm{F}) \mathrm{cc} 1 \\
\mathrm{C}(=\mathrm{O}) \mathrm{N} 1 \mathrm{CC}(\mathrm{F}) \mathrm{CC} 1 \mathrm{C \# N}\end{array}$ & & $\begin{array}{l}\text { Non- } \\
\text { sensitizer }\end{array}$ & & & & & ICCVAM (2013) & \\
\hline $\begin{array}{l}\text { 1,1-Dimethylethyl [(1S)- } \\
\text { 2-[4-[(2-methyl-4- } \\
\text { thiazolyl)methoxy]phen } \\
\text { yl]-1-(2S)- } \\
\text { oxiranylethyl]- } \\
\text { carbamate }\end{array}$ & $\begin{array}{l}\mathrm{Cc} 1 \mathrm{nc}(\mathrm{COc} 2 \mathrm{ccc}(\mathrm{CC}(\mathrm{NC}) \\
=0) \mathrm{OC}(\mathrm{C})(\mathrm{C}) \mathrm{C}) \mathrm{C} 3 \mathrm{CO} 3) \mathrm{c} \\
\mathrm{c} 2) \mathrm{cs} 1\end{array}$ & & $\begin{array}{l}\text { Non- } \\
\text { sensitizer }\end{array}$ & & & & & ICCVAM (2013) & \\
\hline $\begin{array}{l}\text { 1,2,3,5,6,7-Hexahydro- } \\
\text { 2-thioxo-4H- } \\
\text { cyclopentapyrimi-din-4- } \\
\text { one }\end{array}$ & $\begin{array}{l}\mathrm{O}=\mathrm{C} 1 \mathrm{NC}(=\mathrm{S}) \mathrm{NC} 2=\mathrm{C} 1 \mathrm{CC} \\
\mathrm{C} 2\end{array}$ & & $\begin{array}{l}\text { Non- } \\
\text { sensitizer }\end{array}$ & & & & & ICCVAM (2013) & \\
\hline 1,2-Dichloropropane & $\mathrm{CC}(\mathrm{Cl}) \mathrm{CCl}$ & & $\begin{array}{l}\text { Non- } \\
\text { sensitizer }\end{array}$ & & & & & REACH (2008-2014) & \\
\hline 1,2-Dimethoxybenzene & COc1ccccc10C & & $\begin{array}{l}\text { Non- } \\
\text { sensitizer }\end{array}$ & & & & & REACH (2008-2014) & \\
\hline 1,2-dibromoethyne & $\mathrm{BrCCBr}$ & & $\begin{array}{l}\text { Non- } \\
\text { sensitizer }\end{array}$ & & & & & REACH (2008-2014) & \\
\hline 1,2-dichloroethane & $\mathrm{ClCCCl}$ & & $\begin{array}{l}\text { Non- } \\
\text { sensitizer }\end{array}$ & & & & & REACH (2008-2014) & \\
\hline $\begin{array}{l}1,3,5,7,9,11- \\
\text { hexabromocyclododeca } \\
\text { ne }\end{array}$ & $\begin{array}{l}\mathrm{BrC1CC}(\mathrm{Br}) \mathrm{CC}(\mathrm{Br}) \mathrm{CC}(\mathrm{Br} \\
) \mathrm{CC}(\mathrm{Br}) \mathrm{CC}(\mathrm{Br}) \mathrm{C1}\end{array}$ & & $\begin{array}{l}\text { Non- } \\
\text { sensitizer }\end{array}$ & & & & & REACH (2008-2014) & \\
\hline $\begin{array}{l}\text { 1,3,5-tris(2- } \\
\text { hydroxyethyl)-1,3,5- } \\
\text { triazinane-2,4,6-trione }\end{array}$ & $\begin{array}{l}\text { OCCN1C(=O)N(CCO)Cl } \\
=0) \mathrm{N}(\mathrm{CCO}) \mathrm{C} 1=\mathrm{O}\end{array}$ & & $\begin{array}{l}\text { Non- } \\
\text { sensitizer }\end{array}$ & & & & & REACH (2008-2014) & \\
\hline
\end{tabular}




\begin{tabular}{|c|c|c|c|c|c|c|c|c|c|}
\hline Compound name & SMILES & $\begin{array}{l}\text { Human } \\
\text { result }\end{array}$ & $\begin{array}{l}\text { LLNA } \\
\text { result }\end{array}$ & DPRA & KeratinoSens & h-CLAT & $\begin{array}{l}\text { Human } \\
\text { reference }\end{array}$ & LLNA reference & Non-animal reference \\
\hline $\begin{array}{l}\text { 1,3,7-trimethylpurine- } \\
\text { 2,6-dione }\end{array}$ & $\begin{array}{l}\mathrm{CN} 1 \mathrm{c} 2 \mathrm{ncn}(\mathrm{C}) \mathrm{c} 2 \mathrm{C}(=\mathrm{O}) \mathrm{N} \\
(\mathrm{C}) \mathrm{C} 1=\mathrm{O}\end{array}$ & & $\begin{array}{l}\text { Non- } \\
\text { sensitizer }\end{array}$ & & & & & REACH (2008-2014) & \\
\hline $\begin{array}{l}\text { 1,3-Dihydro-7,8- } \\
\text { dimethoxy-2H-3- } \\
\text { benzazepin-2-one }\end{array}$ & $\begin{array}{l}\operatorname{cOc} 1 \mathrm{cc} 2 \mathrm{CC}(=\mathrm{O}) \mathrm{NC}=\mathrm{Cc} \\
2 \mathrm{cc} 10 \mathrm{C}\end{array}$ & & $\begin{array}{l}\text { Non- } \\
\text { sensitizer }\end{array}$ & & & & & REACH (2008-2014) & \\
\hline $\begin{array}{l}\text { 1,3-Dihydroxypropan-2- } \\
\text { one }\end{array}$ & $\mathrm{OCC}(=\mathrm{O}) \mathrm{CO}$ & & $\begin{array}{l}\text { Non- } \\
\text { sensitizer }\end{array}$ & & & & & REACH (2008-2014) & \\
\hline $\begin{array}{l}\text { 1,3- } \\
\text { Dimethylimidazolidin-2- } \\
\text { one }\end{array}$ & $\mathrm{CN} 1 \mathrm{CCN}(\mathrm{C}) \mathrm{C} 1=\mathrm{O}$ & & $\begin{array}{l}\text { Non- } \\
\text { sensitizer }\end{array}$ & & & & & REACH (2008-2014) & \\
\hline 1,3-Dimethylurea & $\mathrm{CNC}(=\mathrm{O}) \mathrm{NC}$ & & $\begin{array}{l}\text { Non- } \\
\text { sensitizer }\end{array}$ & & & & & REACH (2008-2014) & \\
\hline 1,3-Dioxolane & $\mathrm{C} 1 \mathrm{COCO} 1$ & & $\begin{array}{l}\text { Non- } \\
\text { sensitizer }\end{array}$ & & & & & REACH (2008-2014) & \\
\hline 1,3-benzothiazole & c1nc2ccccc2s1 & & $\begin{array}{l}\text { Non- } \\
\text { sensitizer }\end{array}$ & & & & & REACH (2008-2014) & \\
\hline $\begin{array}{l}\text { 1,3-dimethyl-1,3- } \\
\text { diazinan-2-one }\end{array}$ & $\mathrm{CN} 1 \mathrm{CCCN}(\mathrm{C}) \mathrm{C} 1=0$ & & $\begin{array}{l}\text { Non- } \\
\text { sensitizer }\end{array}$ & & & & & REACH (2008-2014) & \\
\hline 1,4-Diethylbenzene & $\operatorname{cCc} 1 \mathrm{ccc}(\mathrm{CC}) \mathrm{cc} 1$ & & $\begin{array}{l}\text { Non- } \\
\text { sensitizer }\end{array}$ & & & & & REACH (2008-2014) & \\
\hline $\begin{array}{l}\text { 1,4- } \\
\text { Dioxacycloheptadecane } \\
\text {-5,17-dione }\end{array}$ & $\begin{array}{l}\text { O=C1CccccccccccC }(= \\
\text { 0) } 0 \text { occo } 1\end{array}$ & & $\begin{array}{l}\text { Non- } \\
\text { sensitizer }\end{array}$ & & & & & REACH (2008-2014) & \\
\hline $\begin{array}{l}\text { 1,4-bis(2-tert- } \\
\text { butylperoxypropan-2- } \\
\text { yl)benzene }\end{array}$ & $\begin{array}{l}\mathrm{CC}(\mathrm{C})(\mathrm{C}) \mathrm{OOC}(\mathrm{C})(\mathrm{C}) \mathrm{c} 1 \mathrm{cc} \\
\mathrm{c}(\mathrm{cc} 1) \mathrm{C}(\mathrm{C})(\mathrm{C}) \mathrm{OOC}(\mathrm{C})(\mathrm{C}) \\
\mathrm{C}\end{array}$ & & $\begin{array}{l}\text { Non- } \\
\text { sensitizer }\end{array}$ & & & & & REACH (2008-2014) & \\
\hline $\begin{array}{l}\text { 1,4-bis[(2,4,6- } \\
\text { trimethylphenyl)amino] } \\
\text { anthracene-9,10-dione }\end{array}$ & $\begin{array}{l}\mathrm{Cc} 1 \mathrm{cc}(\mathrm{C}) \mathrm{c}(\mathrm{Nc} 2 \mathrm{ccc}(\mathrm{Nc} 3 \mathrm{c} \\
(\mathrm{C}) \mathrm{cc}(\mathrm{C}) \mathrm{cc} 3 \mathrm{C}) \mathrm{c} 3 \mathrm{C}(=\mathrm{O}) \mathrm{c} \\
4 \mathrm{ccccc} 4 \mathrm{C}(=\mathrm{O}) \mathrm{c} 23) \mathrm{c}(\mathrm{C}) \mathrm{c} \\
1\end{array}$ & & $\begin{array}{l}\text { Non- } \\
\text { sensitizer }\end{array}$ & & & & & REACH (2008-2014) & \\
\hline $\begin{array}{l}\text { 1-(2',3',4',5'- } \\
\text { Tetramethylphenyl)-3- } \\
\text { (4'-tetrabutylphenyl)- } \\
\text { propane-1,3-dione }\end{array}$ & $\begin{array}{l}\operatorname{Cc} 1 c c(C(O)=C C(=O) c 2 c \\
\operatorname{cc}(c c 2) C(C)(C) C) c(C) c(C \\
) c 1 C\end{array}$ & & $\begin{array}{l}\text { Non- } \\
\text { sensitizer }\end{array}$ & & & & & ICCVAM (2013) & \\
\hline $\begin{array}{l}\text { 1-(3',4',5'- } \\
\text { Tetramethoxyphenyl)- } \\
\text { 4-dimethylpentane-1,3- } \\
\text { dione }\end{array}$ & $\begin{array}{l}\operatorname{cOc} 1 \mathrm{cc}(\mathrm{cc}(\mathrm{OC}) \mathrm{c} 1 \mathrm{OC}) \mathrm{C}( \\
=\mathrm{O}) \mathrm{C}=\mathrm{C}(\mathrm{O}) \mathrm{C}(\mathrm{C})(\mathrm{C}) \mathrm{C}\end{array}$ & & $\begin{array}{l}\text { Non- } \\
\text { sensitizer }\end{array}$ & & & & & ICCVAM (2013) & \\
\hline $\begin{array}{l}\text { 1-(4-chlorophenoxy)-1- } \\
\text { imidazol-1-yl-3,3- } \\
\text { dimethylbutan-2-one }\end{array}$ & $\begin{array}{l}\mathrm{CC}(\mathrm{C})(\mathrm{C}) \mathrm{C}(=\mathrm{O}) \mathrm{C}(\mathrm{Oc} 1 \mathrm{ccc} \\
(\mathrm{Cl}) \mathrm{cc} 1) \mathrm{n} 1 \mathrm{ccnc} 1\end{array}$ & & $\begin{array}{l}\text { Non- } \\
\text { sensitizer }\end{array}$ & & & & & REACH (2008-2014) & \\
\hline $\begin{array}{l}\text { 1-(4-ethoxy-phenyl)-2- } \\
\text { [4-(methyl- }\end{array}$ & $\begin{array}{l}\operatorname{CCOc1ccc}(\operatorname{cc} 1) C(=0) C c \\
1 \operatorname{ccc}(\operatorname{cc} 1) S(C)(=0)=0\end{array}$ & & $\begin{array}{l}\text { Non- } \\
\text { sensitizer }\end{array}$ & & & & & ICCVAM (2013) & \\
\hline
\end{tabular}




\begin{tabular}{|c|c|c|c|c|c|c|c|c|c|}
\hline $\begin{array}{l}\text { sulfonyl)phenyl]- } \\
\text { ethanone }\end{array}$ & & & & & & & & & \\
\hline Compound name & SMILES & $\begin{array}{l}\text { Human } \\
\text { result }\end{array}$ & $\begin{array}{l}\text { LLNA } \\
\text { result }\end{array}$ & DPRA & KeratinoSens & h-CLAT & $\begin{array}{l}\text { Human } \\
\text { reference }\end{array}$ & LLNA reference & Non-animal reference \\
\hline $\begin{array}{l}\text { 1-(4-\{[4,5-dihydroxy-6- } \\
\text { (hydroxymethyl)-3- } \\
{[(3,4,5 \text {-trihydroxy-6- }} \\
\text { methyloxan-2- } \\
\text { yl)oxy]oxan-2-yl]oxy\}- } \\
\text { 2,6-dihydroxyphenyl)-3- } \\
\text { (4- } \\
\text { hydroxyphenyl)propan- } \\
\text { 1-one }\end{array}$ & $\begin{array}{l}\mathrm{CC} 10 \mathrm{OC}(\mathrm{OC} 2 \mathrm{C}(\mathrm{O}) \mathrm{C}(\mathrm{O}) \mathrm{Cl} \\
\mathrm{CO}) \mathrm{OC} 2 \mathrm{Oc} 2 \mathrm{cc}(\mathrm{O}) \mathrm{c}(\mathrm{C}(= \\
\mathrm{O}) \mathrm{CC} 3 \mathrm{ccc}(\mathrm{O}) \mathrm{cc} 3) \mathrm{c}(\mathrm{O}) \mathrm{c} \\
2) \mathrm{C}(0) \mathrm{C}(0) \mathrm{C} 1 \mathrm{O}\end{array}$ & & $\begin{array}{l}\text { Non- } \\
\text { sensitizer }\end{array}$ & & & & & REACH (2008-2014) & \\
\hline 1-Bromononane & $\mathrm{CCCCCCCCCBr}$ & & $\begin{array}{l}\text { Non- } \\
\text { sensitizer }\end{array}$ & & & & & ICCVAM (2013) & \\
\hline $\begin{array}{l}\text { 1-Chloro-3- } \\
\text { iodopropane }\end{array}$ & $\mathrm{ClCCCl}$ & & $\begin{array}{l}\text { Non- } \\
\text { sensitizer }\end{array}$ & & & & & ICCVAM (2013) & \\
\hline 1-Chlorononane & cccccccccCl & & $\begin{array}{l}\text { Non- } \\
\text { sensitizer }\end{array}$ & & & & & ICCVAM (2013) & \\
\hline 1-Ethylpyrrolidin-2-one & CCN1CCCC1=0 & & $\begin{array}{l}\text { Non- } \\
\text { sensitizer }\end{array}$ & & & & & REACH (2008-2014) & \\
\hline 1-lodooctadecane & $\begin{array}{l}\text { CCCCCCCCCCCCCCCCC } \\
\mathrm{Cl}\end{array}$ & & $\begin{array}{l}\text { Non- } \\
\text { sensitizer }\end{array}$ & & & & & ICCVAM (2013) & \\
\hline $\begin{array}{l}\text { 1-[(2-chloro-4- } \\
\text { nitrophenyl)hydrazinyli } \\
\text { dene]naphthalen-2-one }\end{array}$ & $\begin{array}{l}\operatorname{Clc} 1 \mathrm{cc}(\operatorname{ccc} 1 \mathrm{NN}=\mathrm{C} 1 \mathrm{C}(= \\
\mathrm{O}) \mathrm{C}=\mathrm{Cc} 2 \operatorname{ccccc} 12) \mathrm{N}(=0) \\
=\mathrm{O}\end{array}$ & & $\begin{array}{l}\text { Non- } \\
\text { sensitizer }\end{array}$ & & & & & REACH (2008-2014) & \\
\hline $\begin{array}{l}\text { 1-[3-(Cyclopentyl-oxy)- } \\
\text { 4-methoxy-phenyl]-4- } \\
\text { oxocyclohexane } \\
\text { carbonitrile }\end{array}$ & $\begin{array}{l}\text { COc1ccc(cc10C1CCCC1 } \\
) \mathrm{C} 1(\mathrm{CCC}(=0) \mathrm{CC} 1) \mathrm{C \# N}\end{array}$ & & $\begin{array}{l}\text { Non- } \\
\text { sensitizer }\end{array}$ & & & & & ICCVAM (2013) & \\
\hline $\begin{array}{l}\text { 1-[4-[2-[4-(2- } \\
\text { hydroxypropoxy)phenyl } \\
\text { ]propan-2- } \\
\text { yl]phenoxy]propan-2-ol }\end{array}$ & $\begin{array}{l}\mathrm{CC}(\mathrm{O}) \operatorname{coc} 1 \mathrm{ccc}(\mathrm{cc} 1) \mathrm{C}(\mathrm{C}) \\
(\mathrm{C}) \mathrm{c} 1 \mathrm{ccc}(\mathrm{OCC}(\mathrm{C}) \mathrm{O}) \mathrm{cc} 1\end{array}$ & & $\begin{array}{l}\text { Non- } \\
\text { sensitizer }\end{array}$ & & & & & REACH (2008-2014) & \\
\hline $\begin{array}{l}\text { 1-[4-hydroxy-5- } \\
\text { (hydroxymethyl)oxolan- } \\
2-y l]-5- \\
\text { methylpyrimidine-2,4- } \\
\text { dione }\end{array}$ & $\begin{array}{l}\mathrm{CC} 1=\mathrm{CN}(\mathrm{C} 2 \mathrm{CC}(\mathrm{O}) \mathrm{C}(\mathrm{CO}) \\
\mathrm{O} 2) \mathrm{C}(=\mathrm{O}) \mathrm{NC} 1=\mathrm{O}\end{array}$ & & $\begin{array}{l}\text { Non- } \\
\text { sensitizer }\end{array}$ & & & & & REACH (2008-2014) & \\
\hline $\begin{array}{l}\text { 1-[5-[(4- } \\
\text { Fluorophenyl)methyl]- } \\
\text { 2-furanyl]ethanone }\end{array}$ & $\begin{array}{l}\mathrm{CC}(=0) \mathrm{c} 1 \mathrm{ccc}(\mathrm{Cc} 2 \mathrm{ccc}(\mathrm{F}) \\
\mathrm{cc} 2) \mathrm{o} 1\end{array}$ & & $\begin{array}{l}\text { Non- } \\
\text { sensitizer }\end{array}$ & & & & & ICCVAM (2013) & \\
\hline $\begin{array}{l}\text { 1-amino-4-(4-amino- } \\
\text { 9,10-dioxoanthracen-1- } \\
\text { yl)anthracene-9,10- } \\
\text { dione }\end{array}$ & $\begin{array}{l}\text { Nc1ccc(- } \\
\mathrm{c} 2 \operatorname{ccc}(\mathrm{N}) \mathrm{c3C}(=\mathrm{O}) \mathrm{c} 4 \mathrm{cccc} \\
\mathrm{c} 4 \mathrm{C}(=\mathrm{O}) \mathrm{c} 23) \mathrm{c} 2 \mathrm{C}(=\mathrm{O}) \mathrm{c3} \\
\operatorname{ccccc} 3 \mathrm{C}(=\mathrm{O}) \mathrm{c} 12\end{array}$ & & $\begin{array}{l}\text { Non- } \\
\text { sensitizer }\end{array}$ & & & & & REACH (2008-2014) & \\
\hline
\end{tabular}




\begin{tabular}{|c|c|c|c|c|c|c|c|c|c|}
\hline $\begin{array}{l}\text { 1-chloro-2-(2- } \\
\text { chloroethoxymethoxy)e } \\
\text { thane }\end{array}$ & $\mathrm{ClCCOCOCCCl}$ & & $\begin{array}{l}\text { Non- } \\
\text { sensitizer }\end{array}$ & & & & & REACH (2008-2014) & \\
\hline Compound name & SMILES & $\begin{array}{l}\text { Human } \\
\text { result }\end{array}$ & $\begin{array}{l}\text { LLNA } \\
\text { result }\end{array}$ & DPRA & KeratinoSens & h-CLAT & $\begin{array}{l}\text { Human } \\
\text { reference }\end{array}$ & LLNA reference & Non-animal reference \\
\hline $\begin{array}{l}\text { 1-chloro-4-(4- } \\
\text { chlorophenyl)sulfonylb } \\
\text { enzene }\end{array}$ & $\begin{array}{l}\text { Clc1ccc(cc1)S(=0)(=O)c } \\
1 \operatorname{ccc}(\mathrm{Cl}) \operatorname{cc} 1\end{array}$ & & $\begin{array}{l}\text { Non- } \\
\text { sensitizer }\end{array}$ & & & & & REACH (2008-2014) & \\
\hline $\begin{array}{l}\text { 1- } \\
\text { dimethoxyphosphorylpr } \\
\text { opane }\end{array}$ & $\mathrm{CCCP}(=\mathrm{O})(\mathrm{OC}) \mathrm{OC}$ & & $\begin{array}{l}\text { Non- } \\
\text { sensitizer }\end{array}$ & & & & & REACH (2008-2014) & \\
\hline 1-ethenylimidazole & $\mathrm{C}=\mathrm{Cn} 1 \mathrm{ccnc} 1$ & & $\begin{array}{l}\text { Non- } \\
\text { sensitizer }\end{array}$ & & & & & REACH (2008-2014) & \\
\hline $\begin{array}{l}\text { 1-hydroxypropan-2-yl } \\
\text { 2-methylprop-2-enoate }\end{array}$ & $\mathrm{CC}(\mathrm{CO}) \mathrm{OC}(=\mathrm{O}) \mathrm{C}(\mathrm{C})=\mathrm{C}$ & & $\begin{array}{l}\text { Non- } \\
\text { sensitizer }\end{array}$ & & & & & REACH (2008-2014) & \\
\hline $\begin{array}{l}\text { 1-methoxy-2-(2- } \\
\text { methoxyethoxymethox } \\
\text { y)ethane }\end{array}$ & COCCOCOCCOC & & $\begin{array}{l}\text { Non- } \\
\text { sensitizer }\end{array}$ & & & & & REACH (2008-2014) & \\
\hline $\begin{array}{l}\text { 1- } \\
\text { octadecyldisulfanylocta } \\
\text { decane }\end{array}$ & $\begin{array}{l}\text { CCCCCCCCCCCCCCCCCC } \\
\text { CSSCCCCCCCCCCCCCCC } \\
\text { CCCC }\end{array}$ & & $\begin{array}{l}\text { Non- } \\
\text { sensitizer }\end{array}$ & & & & & REACH (2008-2014) & \\
\hline $\begin{array}{l}\text { 10-hydroxy-11a- } \\
\text { methyl- } \\
1 \mathrm{H}, 2 \mathrm{H}, 3 \mathrm{H}, 3 \mathrm{aH}, 3 \mathrm{bH}, 4 \mathrm{H}, 5 \\
\mathrm{H}, 7 \mathrm{H}, 8 \mathrm{H}, 9 \mathrm{H}, 9 \mathrm{aH}, 9 \mathrm{bH}, 10 \\
\mathrm{H}, 11 \mathrm{H}, 11 \mathrm{aH}- \\
\text { cyclopenta[a]phenanthr } \\
\text { ene-1,7-dione }\end{array}$ & $\begin{array}{l}\mathrm{CC} 12 \mathrm{CC}(0) \mathrm{C} 3 \mathrm{C}(\mathrm{CCC} 4= \\
\mathrm{CC}(=0) \mathrm{CCC} 34) \mathrm{C} 1 \mathrm{CCC} 2= \\
\mathrm{O}\end{array}$ & & $\begin{array}{l}\text { Non- } \\
\text { sensitizer }\end{array}$ & & & & & REACH (2008-2014) & \\
\hline $\begin{array}{l}\text { 17,21-dihydroxy-16?- } \\
\text { methylpregna- } \\
\text { 1,4,9(11)-triene-3,20- } \\
\text { dione }\end{array}$ & $\begin{array}{l}\mathrm{CC} 1 \mathrm{CC} 2 \mathrm{C} 3 \mathrm{CCC} 4=\mathrm{CC}(=\mathrm{O} \\
) \mathrm{C}=\mathrm{CC} 4(\mathrm{C}) \mathrm{C} 3=\mathrm{CCC} 2(\mathrm{C}) \mathrm{C} \\
1(\mathrm{O}) \mathrm{C}(=0) \mathrm{CO}\end{array}$ & & $\begin{array}{l}\text { Non- } \\
\text { sensitizer }\end{array}$ & & & & & ICCVAM (2013) & \\
\hline 1H-1,2,4-Triazole & $\mathrm{c} 1 \mathrm{nnc}[\mathrm{nH}] 1$ & & $\begin{array}{l}\text { Non- } \\
\text { sensitizer }\end{array}$ & & & & & REACH (2008-2014) & \\
\hline 2'-4-Dihydroxychalcone & $\begin{array}{l}\text { Oc1ccc }(\mathrm{C}=\mathrm{CC}(=0) \mathrm{c} 2 \mathrm{ccc} \\
\mathrm{cc} 2 \mathrm{O}) \mathrm{cc1}\end{array}$ & & $\begin{array}{l}\text { Non- } \\
\text { sensitizer }\end{array}$ & & & & & ICCVAM (2013) & \\
\hline 2,2'-Oxybisethanol & occocco & & $\begin{array}{l}\text { Non- } \\
\text { sensitizer }\end{array}$ & & & & & ICCVAM (2013) & \\
\hline $\begin{array}{l}\text { 2,2,2- } \\
\text { TRIFLUOROETHANOL }\end{array}$ & $\mathrm{OCC}(\mathrm{F})(\mathrm{F}) \mathrm{F}$ & & $\begin{array}{l}\text { Non- } \\
\text { sensitizer }\end{array}$ & & & & & REACH (2008-2014) & \\
\hline $\begin{array}{l}\text { 2,2,3,3- } \\
\text { tetrafluorooxetane }\end{array}$ & $\mathrm{FC} 1(\mathrm{~F}) \mathrm{COC} 1(\mathrm{~F}) \mathrm{F}$ & & $\begin{array}{l}\text { Non- } \\
\text { sensitizer }\end{array}$ & & & & & REACH (2008-2014) & \\
\hline $\begin{array}{l}\text { 2,2-Dimethylpropane- } \\
\text { 1,3-diol }\end{array}$ & $\mathrm{CC}(\mathrm{C})(\mathrm{CO}) \mathrm{CO}$ & & $\begin{array}{l}\text { Non- } \\
\text { sensitizer }\end{array}$ & & & & & REACH (2008-2014) & \\
\hline
\end{tabular}




\begin{tabular}{|c|c|c|c|c|c|c|c|c|c|}
\hline Compound name & SMILES & $\begin{array}{l}\text { Human } \\
\text { result }\end{array}$ & $\begin{array}{l}\text { LLNA } \\
\text { result }\end{array}$ & DPRA & KeratinoSens & h-CLAT & $\begin{array}{l}\text { Human } \\
\text { reference }\end{array}$ & LLNA reference & Non-animal reference \\
\hline $\begin{array}{l}\text { 2,2- } \\
\text { bis(hydroxymethyl)prop } \\
\text { ane-1,3-diol; 3-[3-(3- } \\
\text { hydroxypropoxy)-2,2- } \\
\text { bis(3- } \\
\text { hydroxypropoxymethyl) } \\
\text { propoxy]propan-1-ol }\end{array}$ & $\begin{array}{l}\text { OCCCOCC }(\mathrm{COCCCO})(\mathrm{C} \\
\text { ocCCO)COCCCO }\end{array}$ & & $\begin{array}{l}\text { Non- } \\
\text { sensitizer }\end{array}$ & & & & & REACH (2008-2014) & \\
\hline $\begin{array}{l}\text { 2,3,3,3-tetrafluoro-2- } \\
(1,1,2,2,3,3,3- \\
\text { heptafluoropropoxy)pr } \\
\text { opanoic acid }\end{array}$ & $\begin{array}{l}O C(=O) C(F)(O C(F)(F) C( \\
F)(F) C(F)(F) F) C(F)(F) F\end{array}$ & & $\begin{array}{l}\text { Non- } \\
\text { sensitizer }\end{array}$ & & & & & REACH (2008-2014) & \\
\hline $\begin{array}{l}\text { 2,3,4,5-tetrahydro-n-(1- } \\
\text { methylethyl)-2,4-dioxo- } \\
\text { n,5-diphenyl-3- } \\
\text { [(phenylmethoxy)- } \\
\text { imino]-1h-1,5- } \\
\text { benzodiazepine-1- } \\
\text { acetamide }\end{array}$ & $\begin{array}{l}\mathrm{CC}(\mathrm{C}) \mathrm{N}(\mathrm{C}(=\mathrm{O}) \mathrm{CN} 1 \mathrm{c} 2 \mathrm{ccc} \\
\mathrm{cc} 2 \mathrm{~N}(\mathrm{c} 2 \mathrm{ccccc} 2) \mathrm{C}(=\mathrm{O}) \mathrm{C}( \\
=\mathrm{NOC} 2 \mathrm{ccccc} 2) \mathrm{C} 1=\mathrm{O}) \mathrm{c} \\
1 \mathrm{ccccc} 1\end{array}$ & & $\begin{array}{l}\text { Non- } \\
\text { sensitizer }\end{array}$ & & & & & ICCVAM (2013) & \\
\hline $\begin{array}{l}\text { 2,3-Dimethyl-2H- } \\
\text { indazol-6-amine }\end{array}$ & $\operatorname{cc} 1 n(\mathrm{C}) n \mathrm{nc} 2 \mathrm{cc}(\mathrm{N}) \operatorname{ccc} 12$ & & $\begin{array}{l}\text { Non- } \\
\text { sensitizer }\end{array}$ & & & & & ICCVAM (2013) & \\
\hline $\begin{array}{l}\text { 2,4,4-trimethylpentan- } \\
\text { 2-yl 3-(5,5- } \\
\text { dimethylhexyl)dioxiran } \\
\text { e-3-carboxylate }\end{array}$ & $\begin{array}{l}\mathrm{CC}(\mathrm{C})(\mathrm{C}) \mathrm{CCCCC1}(\mathrm{OO} 1) \\
\mathrm{C}(=0) \mathrm{OC}(\mathrm{C})(\mathrm{C}) \mathrm{CC}(\mathrm{C})(\mathrm{C}) \\
\mathrm{C}\end{array}$ & & $\begin{array}{l}\text { Non- } \\
\text { sensitizer }\end{array}$ & & & & & REACH (2008-2014) & \\
\hline $\begin{array}{l}\text { 2,4,5-Trioxa-1-phospha- } \\
\text { 3- } \\
\text { borabicyclo[1.1.1]penta } \\
\text { ne 1-oxide }\end{array}$ & $\mathrm{O}=\mathrm{P} 12 \mathrm{OB}(\mathrm{O} 1) \mathrm{O} 2$ & & $\begin{array}{l}\text { Non- } \\
\text { sensitizer }\end{array}$ & & & & & REACH (2008-2014) & \\
\hline $\begin{array}{l}2,4,7,9- \\
\text { tetramethyldecane-4,7- } \\
\text { diol }\end{array}$ & $\begin{array}{l}\mathrm{CC}(\mathrm{C}) \mathrm{CC}(\mathrm{C})(\mathrm{O}) \mathrm{CCC}(\mathrm{C})(\mathrm{O} \\
) \mathrm{CC}(\mathrm{C}) \mathrm{C}\end{array}$ & & $\begin{array}{l}\text { Non- } \\
\text { sensitizer }\end{array}$ & & & & & REACH (2008-2014) & \\
\hline $\begin{array}{l}\text { 2,6-Dimethoxy-4- } \\
\text { methyl-5-[3- } \\
\text { (trifluoromethyl)- } \\
\text { phenoxy]-8- } \\
\text { quinolinamine }\end{array}$ & $\begin{array}{l}\mathrm{COc} 1 \mathrm{cc}(\mathrm{C}) \mathrm{c} 2 \mathrm{c}(\mathrm{Oc} 3 \mathrm{cccc}( \\
\mathrm{c} 3) \mathrm{C}(\mathrm{F})(\mathrm{F}) \mathrm{F}) \mathrm{c}(\mathrm{OC}) \mathrm{cc}(\mathrm{N}) \\
\mathrm{c} 2 \mathrm{n} 1\end{array}$ & & $\begin{array}{l}\text { Non- } \\
\text { sensitizer }\end{array}$ & & & & & ICCVAM (2013) & \\
\hline 2,6-Dimethylaniline & $\operatorname{Cc} 1 \operatorname{cccc}(\mathrm{C}) \mathrm{c} 1 \mathrm{~N}$ & & $\begin{array}{l}\text { Non- } \\
\text { sensitizer }\end{array}$ & & & & & REACH (2008-2014) & \\
\hline $\begin{array}{l}\text { 2,6-Dimethylbenzoic } \\
\text { acid }\end{array}$ & $\operatorname{Cc} 1 \operatorname{cccc}(\mathrm{C}) \operatorname{c} 1 \mathrm{C}(\mathrm{O})=0$ & & $\begin{array}{l}\text { Non- } \\
\text { sensitizer }\end{array}$ & & & & & ICCVAM (2013) & \\
\hline
\end{tabular}




\begin{tabular}{|l|l|l|l|l|l|l|l|}
\hline $\begin{array}{l}2,9- \\
\text { Dimethylquinacridone }\end{array}$ & $\begin{array}{l}\text { Cc1ccc2Nc3cc4C(=0)c5 } \\
\operatorname{cc}(\mathrm{C}) \operatorname{ccc} 5 \mathrm{Nc4cc3C}(=0) \\
\mathrm{c2c1}\end{array}$ & $\begin{array}{l}\text { Non- } \\
\text { sensitizer }\end{array}$ & & REACH (2008-2014) & & & \\
\hline
\end{tabular}

\begin{tabular}{|c|c|c|c|c|c|c|c|c|c|}
\hline Compound name & SMILES & $\begin{array}{l}\text { Human } \\
\text { result }\end{array}$ & $\begin{array}{l}\text { LLNA } \\
\text { result }\end{array}$ & DPRA & KeratinoSens & h-CLAT & $\begin{array}{l}\text { Human } \\
\text { reference }\end{array}$ & LLNA reference & Non-animal reference \\
\hline $\begin{array}{l}\text { 2,9-dichloro-5,12- } \\
\text { dihydroquino[2,3- } \\
\text { b]acridine-7,14-dione }\end{array}$ & $\begin{array}{l}\text { Clc1ccc2Nc3cc4C(=O)c } \\
5 c c(\mathrm{Cl}) \operatorname{ccc} 5 \mathrm{Nc} 4 \mathrm{cc} 3 \mathrm{C}(= \\
\mathrm{O}) \mathrm{c} 2 \mathrm{c} 1\end{array}$ & & $\begin{array}{l}\text { Non- } \\
\text { sensitizer }\end{array}$ & & & & & REACH (2008-2014) & \\
\hline $\begin{array}{l}\text { 2-(1-amino-1-imino-2- } \\
\text { methylpropan-2- } \\
\text { yl)diazenyl-2- } \\
\text { methylpropanimidamid } \\
\text { e hydrochloride }\end{array}$ & $\begin{array}{l}C C(C)(N=N C(C)(C) C(N)= \\
N) C(N)=N\end{array}$ & & $\begin{array}{l}\text { Non- } \\
\text { sensitizer }\end{array}$ & & & & & REACH (2008-2014) & \\
\hline $\begin{array}{l}\text { 2-(2- } \\
\text { dimethylaminoethoxy)- } \\
\mathrm{N}, \mathrm{N}- \\
\text { dimethylethanamine }\end{array}$ & $\mathrm{CN}(\mathrm{C}) \operatorname{CCOCCN}(\mathrm{C}) \mathrm{C}$ & & $\begin{array}{l}\text { Non- } \\
\text { sensitizer }\end{array}$ & & & & & REACH (2008-2014) & \\
\hline $\begin{array}{l}\text { 2-(2- } \\
\text { ethenoxyethoxy)ethoxy } \\
\text { ethene }\end{array}$ & $\mathrm{C}=\mathrm{COCCOCCOC}=\mathrm{C}$ & & $\begin{array}{l}\text { Non- } \\
\text { sensitizer }\end{array}$ & & & & & REACH (2008-2014) & \\
\hline $\begin{array}{l}\text { 2-(2- } \\
\text { hexoxyethoxy)ethanol }\end{array}$ & cCCCCCOCCOCCO & & $\begin{array}{l}\text { Non- } \\
\text { sensitizer }\end{array}$ & & & & & REACH (2008-2014) & \\
\hline $\begin{array}{l}\text { 2-(2- } \\
\text { hydroxypropoxy)propa } \\
\text { n-1-ol }\end{array}$ & $\mathrm{CC}(\mathrm{O}) \mathrm{COC}(\mathrm{C}) \mathrm{CO}$ & & $\begin{array}{l}\text { Non- } \\
\text { sensitizer }\end{array}$ & & & & & REACH (2008-2014) & \\
\hline $\begin{array}{l}\text { 2-(2-methoxy-4- } \\
\text { nitrophenyl)diazenyl-N- } \\
\text { (2-methoxyphenyl)-3- } \\
\text { oxobutanamide }\end{array}$ & $\begin{array}{l}\operatorname{COc} 1 \operatorname{ccccc} 1 N C(=0) C(= \\
N N \operatorname{Nocc}(\operatorname{cc} 10 C) N(=0) \\
=0) C(C)=0\end{array}$ & & $\begin{array}{l}\text { Non- } \\
\text { sensitizer }\end{array}$ & & & & & REACH (2008-2014) & \\
\hline $\begin{array}{l}\text { 2-(2- } \\
\text { methoxyphenyl)diazeny } \\
\text { I-3-oxo-N-(2-oxo-1,3- } \\
\text { dihydrobenzimidazol-5- } \\
\text { yl)butanamide }\end{array}$ & $\begin{array}{l}\operatorname{COc} 1 \operatorname{ccccc} 1 \mathrm{NN}=\mathrm{C}(\mathrm{C}(\mathrm{C}) \\
=0) \mathrm{C}(=\mathrm{O}) \mathrm{Nc} 1 \mathrm{ccc} 2 \mathrm{NC}(= \\
\mathrm{O}) \mathrm{Nc} 2 \mathrm{c} 1\end{array}$ & & $\begin{array}{l}\text { Non- } \\
\text { sensitizer }\end{array}$ & & & & & REACH (2008-2014) & \\
\hline $\begin{array}{l}\text { 2-(2-phenylpropan-2- } \\
\text { ylperoxy)propan-2- } \\
\text { ylbenzene }\end{array}$ & $\begin{array}{l}\mathrm{CC}(\mathrm{C})(\mathrm{OOC}(\mathrm{C})(\mathrm{C}) \mathrm{c} 1 \mathrm{cccc} \\
\mathrm{c} 1) \mathrm{c} 1 \mathrm{ccccc} 1\end{array}$ & & $\begin{array}{l}\text { Non- } \\
\text { sensitizer }\end{array}$ & & & & & REACH (2008-2014) & \\
\hline $\begin{array}{l}\text { 2-(3- } \\
\text { oxobutanoyloxy)ethyl } \\
\text { 2-methylprop-2-enoate }\end{array}$ & $\begin{array}{l}\mathrm{CC}(=0) \mathrm{CC}(=0) \mathrm{OCCOC}(= \\
\mathrm{O}) \mathrm{C}(\mathrm{C})=\mathrm{C}\end{array}$ & & $\begin{array}{l}\text { Non- } \\
\text { sensitizer }\end{array}$ & & & & & REACH (2008-2014) & \\
\hline $\begin{array}{l}\text { 2-(4-Oxopentyl)-1h- } \\
\text { isoindole-1,3(2h)-dione }\end{array}$ & $\begin{array}{l}\mathrm{CC}(=0) \operatorname{CCCN} 1 \mathrm{C}(=0) \operatorname{coc} \\
\operatorname{ccc} 2 \mathrm{C} 1=0\end{array}$ & & $\begin{array}{l}\text { Non- } \\
\text { sensitizer }\end{array}$ & & & & & ICCVAM (2013) & \\
\hline
\end{tabular}




\begin{tabular}{|c|c|c|c|c|c|c|c|c|c|}
\hline $\begin{array}{l}\text { 2-(4-chloro-2- } \\
\text { nitrophenyl)diazenyl-N- } \\
\text { (2-chlorophenyl)-3- } \\
\text { oxobutanamide } \\
\end{array}$ & $\begin{array}{l}\mathrm{CC}(=\mathrm{O}) \mathrm{C}(=\mathrm{NNc1} \operatorname{ccc}(\mathrm{Cl}) \\
\operatorname{cc} 1 \mathrm{~N}(=\mathrm{O})=\mathrm{O}) \mathrm{C}(=\mathrm{O}) \mathrm{Nc1} \\
\operatorname{ccccc} 1 \mathrm{Cl}\end{array}$ & & $\begin{array}{l}\text { Non- } \\
\text { sensitizer }\end{array}$ & & & & & REACH (2008-2014) & \\
\hline $\begin{array}{l}\text { 2-(4-chloro-2- } \\
\text { nitrophenyl)diazenyl-N- } \\
\text { (2-methoxyphenyl)-3- } \\
\text { oxobutanamide }\end{array}$ & $\begin{array}{l}\operatorname{coc} 1 \operatorname{ccccc} 1 \mathrm{NC}(=0) \mathrm{C}= \\
\mathrm{NNc} 1 \operatorname{ccc}(\mathrm{Cl}) \operatorname{cc} 1 \mathrm{~N}(=0)= \\
\mathrm{O}) \mathrm{C}(\mathrm{C})=0\end{array}$ & & $\begin{array}{l}\text { Non- } \\
\text { sensitizer }\end{array}$ & & & & & REACH (2008-2014) & \\
\hline Compound name & SMILES & $\begin{array}{l}\text { Human } \\
\text { result }\end{array}$ & $\begin{array}{l}\text { LLNA } \\
\text { result }\end{array}$ & DPRA & KeratinoSens & h-CLAT & $\begin{array}{l}\text { Human } \\
\text { reference }\end{array}$ & LLNA reference & Non-animal reference \\
\hline $\begin{array}{l}\text { 2-(4-ethoxyphenyl)-3- } \\
\text { [4-(methyl- } \\
\text { sulfonyl)phenyl- } \\
\text { ]pyrazolo[1,5-b]- } \\
\text { pyridazine }\end{array}$ & $\begin{array}{l}\operatorname{ccOc} 1 \operatorname{ccc}(\operatorname{cc} 1)- \\
\mathrm{c} 1 \mathrm{nn} 2 \mathrm{ncccc} 2 \mathrm{c} 1- \\
\operatorname{c1ccc}(\operatorname{cc} 1) \mathrm{S}(\mathrm{C})(=0)=0\end{array}$ & & $\begin{array}{l}\text { Non- } \\
\text { sensitizer }\end{array}$ & & & & & ICCVAM (2013) & \\
\hline $\begin{array}{l}\text { 2-(4-methoxy-2- } \\
\text { nitrophenyl)diazenyl-N- } \\
\text { (2-methoxyphenyl)-3- } \\
\text { oxobutanamide }\end{array}$ & $\begin{array}{l}\operatorname{COc1ccc}(N N=C(C(C)=0 \\
C(=0) N c 2 \operatorname{ccccc} 2 O C) c( \\
c 1) N(=0)=0\end{array}$ & & $\begin{array}{l}\text { Non- } \\
\text { sensitizer }\end{array}$ & & & & & REACH (2008-2014) & \\
\hline $\begin{array}{l}\text { 2-(4-methyl-1-cyclohex- } \\
\text { 3-enyl)propan-2-yl } \\
\text { acetate }\end{array}$ & $\begin{array}{l}\mathrm{CC}(=0) \mathrm{OC}(\mathrm{C})(\mathrm{C}) \mathrm{C} 1 \mathrm{CCC}( \\
\mathrm{C})=\mathrm{CC} 1\end{array}$ & & $\begin{array}{l}\text { Non- } \\
\text { sensitizer }\end{array}$ & & & & & REACH (2008-2014) & \\
\hline $\begin{array}{l}\text { 2-(4-methyl-2- } \\
\text { nitrophenyl)diazenyl-3- } \\
\text { oxo- } \mathrm{N} \text { - } \\
\text { phenylbutanamide }\end{array}$ & $\begin{array}{l}\mathrm{CC}(=0) \mathrm{C}(=\mathrm{NN} 1 \mathrm{ccc}(\mathrm{C}) \mathrm{c} \\
\mathrm{c} 1 \mathrm{~N}(=\mathrm{O})=0) \mathrm{C}(=\mathrm{O}) \mathrm{Nc} 1 \mathrm{c} \\
\operatorname{cccc1}\end{array}$ & & $\begin{array}{l}\text { Non- } \\
\text { sensitizer }\end{array}$ & & & & & REACH (2008-2014) & \\
\hline $\begin{array}{l}\text { 2-(4- } \\
\text { nitrophenyl)diazenyl-3- } \\
\text { oxo-N-(2-oxo-1,3- } \\
\text { dihydrobenzimidazol-5- } \\
\text { yl)butanamide }\end{array}$ & $\begin{array}{l}\mathrm{CC}(=\mathrm{O}) \mathrm{C}(=\mathrm{NNc} 1 \mathrm{ccc}(\mathrm{cc} 1 \\
) \mathrm{N}(=\mathrm{O})=\mathrm{O}) \mathrm{C}(=0) \mathrm{Nc} 1 \mathrm{ccc} \\
2 \mathrm{NC}(=0) \mathrm{Nc} 2 \mathrm{c1}\end{array}$ & & $\begin{array}{l}\text { Non- } \\
\text { sensitizer }\end{array}$ & & & & & REACH (2008-2014) & \\
\hline $\begin{array}{l}\text { 2-(benzyl)tert- } \\
\text { butyl)amino)-1-( } \alpha, 4- \\
\text { dihydroxy-m- } \\
\text { tolyl)ethane }\end{array}$ & $\begin{array}{l}\mathrm{CC}(\mathrm{C})(\mathrm{C}) \mathrm{N}(\mathrm{CC}(\mathrm{O}) \mathrm{c} 1 \mathrm{ccc}( \\
\mathrm{O}) \mathrm{c}(\mathrm{CO}) \mathrm{c} 1) \mathrm{Cc} 1 \mathrm{ccccc} 1\end{array}$ & & $\begin{array}{l}\text { Non- } \\
\text { sensitizer }\end{array}$ & & & & & ICCVAM (2013) & \\
\hline $\begin{array}{l}2- \\
\text { (chloromethyl)oxirane; } \\
\text { 2,6-dibromo-4-[2-(3,5- } \\
\text { dibromo-4- } \\
\text { hydroxyphenyl)propan- } \\
\text { 2-yl]phenol }\end{array}$ & $\begin{array}{l}\mathrm{CC}(\mathrm{C})(\mathrm{c} 1 \mathrm{cc}(\mathrm{Br}) \mathrm{c}(\mathrm{O}) \mathrm{c}(\mathrm{Br}) \\
\mathrm{c} 1) \mathrm{c} 1 \mathrm{cc}(\mathrm{Br}) \mathrm{c}(\mathrm{O}) \mathrm{c}(\mathrm{Br}) \mathrm{c1}\end{array}$ & & $\begin{array}{l}\text { Non- } \\
\text { sensitizer }\end{array}$ & & & & & REACH (2008-2014) & \\
\hline $\begin{array}{l}2- \\
\text { (phenoxy)ethoxybenze } \\
\text { ne }\end{array}$ & $\begin{array}{l}\mathrm{C}(\operatorname{COc} 1 \mathrm{ccccc} 1) \operatorname{Oc} 1 \mathrm{cccc} \\
\mathrm{c1}\end{array}$ & & $\begin{array}{l}\text { Non- } \\
\text { sensitizer }\end{array}$ & & & & & REACH (2008-2014) & \\
\hline $\begin{array}{l}\text { 2-(phenoxy)ethyl 2- } \\
\text { methylpropanoate }\end{array}$ & $\begin{array}{l}\mathrm{CC}(\mathrm{C}) \mathrm{C}(=0) 0 \mathrm{OCCOc} 1 \mathrm{ccc} \\
\mathrm{cc1}\end{array}$ & & $\begin{array}{l}\text { Non- } \\
\text { sensitizer }\end{array}$ & & & & & REACH (2008-2014) & \\
\hline
\end{tabular}




\begin{tabular}{|c|c|c|c|c|c|c|c|c|c|}
\hline $\begin{array}{l}\text { 2-Amino-3- } \\
\text { methylbutanoic acid }\end{array}$ & $\mathrm{CC}(\mathrm{C}) \mathrm{C}(\mathrm{N}) \mathrm{C}(\mathrm{O})=\mathrm{O}$ & & $\begin{array}{l}\text { Non- } \\
\text { sensitizer }\end{array}$ & & & & & REACH (2008-2014) & \\
\hline 2-Aminoacetic acid & $\mathrm{NCC}(\mathrm{O})=\mathrm{O}$ & & $\begin{array}{l}\text { Non- } \\
\text { sensitizer }\end{array}$ & & & & & REACH (2008-2014) & \\
\hline $\begin{array}{l}\text { 2-Aminoethyl- } \\
\text { methylsulfone }\end{array}$ & $\mathrm{CS}(=\mathrm{O})(=\mathrm{O}) \mathrm{CCN}$ & & $\begin{array}{l}\text { Non- } \\
\text { sensitizer }\end{array}$ & & & & & ICCVAM (2013) & \\
\hline $\begin{array}{l}\text { 2-Bromo-5- } \\
\text { propoxybenzoic acid }\end{array}$ & $\begin{array}{l}\operatorname{CCCOc} 1 \mathrm{ccc}(\mathrm{Br}) \mathrm{c}(\mathrm{c} 1) \mathrm{C}( \\
\mathrm{O})=0\end{array}$ & & $\begin{array}{l}\text { Non- } \\
\text { sensitizer }\end{array}$ & & & & & ICCVAM (2013) & \\
\hline Compound name & SMILES & $\begin{array}{l}\text { Human } \\
\text { result }\end{array}$ & $\begin{array}{l}\text { LLNA } \\
\text { result }\end{array}$ & DPRA & KeratinoSens & h-CLAT & $\begin{array}{l}\text { Human } \\
\text { reference }\end{array}$ & LLNA reference & Non-animal reference \\
\hline $\begin{array}{l}\text { 2-Chloro-6-methoxy-4- } \\
\text { methylquinoline }\end{array}$ & $\begin{array}{l}\text { COc1ccc2nc(Cl)cc(C)c2 } \\
\text { c1 }\end{array}$ & & $\begin{array}{l}\text { Non- } \\
\text { sensitizer }\end{array}$ & & & & & ICCVAM (2013) & \\
\hline 2-Chloro-6-nitrotoluene & $\operatorname{Cc} 1 \mathrm{c}(\mathrm{Cl}) \operatorname{ccc} 1 \mathrm{~N}(=\mathrm{O})=0$ & & $\begin{array}{l}\text { Non- } \\
\text { sensitizer }\end{array}$ & & & & & ICCVAM (2013) & \\
\hline 2-Chloroethanol & $\mathrm{OCCCl}$ & & $\begin{array}{l}\text { Non- } \\
\text { sensitizer }\end{array}$ & & & & & REACH (2008-2014) & \\
\hline 2-Ethylhexyl benzoate & $\begin{array}{l}\operatorname{ccccc}(\operatorname{CC}) \operatorname{cOC}(=0) \operatorname{c1c} \\
\operatorname{cccc} 1\end{array}$ & & $\begin{array}{l}\text { Non- } \\
\text { sensitizer }\end{array}$ & & & & & REACH (2008-2014) & \\
\hline $\begin{array}{l}\text { 2-Hydroxy-N,N- } \\
\text { dimethylpropanamide }\end{array}$ & $\mathrm{CC}(\mathrm{O}) \mathrm{C}(=\mathrm{O}) \mathrm{N}(\mathrm{C}) \mathrm{C}$ & & $\begin{array}{l}\text { Non- } \\
\text { sensitizer }\end{array}$ & & & & & REACH (2008-2014) & \\
\hline $\begin{array}{l}2- \\
\text { Hydroxybenzothiazole }\end{array}$ & $\mathrm{O}=\mathrm{C} 1 \mathrm{Nc} 2 \operatorname{ccccc} 2 \mathrm{~S} 1$ & & $\begin{array}{l}\text { Non- } \\
\text { sensitizer }\end{array}$ & & & & & ICCVAM (2013) & \\
\hline $\begin{array}{l}\text { 2- } \\
\text { Mercaptobenzoxazole }\end{array}$ & $\mathrm{S}=\mathrm{C} 1 \mathrm{Nc} 2 \operatorname{ccccc} 2 \mathrm{O} 1$ & & $\begin{array}{l}\text { Non- } \\
\text { sensitizer }\end{array}$ & & & & & ICCVAM (2013) & \\
\hline 2-Methylbutan-2-ol & $\mathrm{CCC}(\mathrm{C})(\mathrm{C}) \mathrm{O}$ & & $\begin{array}{l}\text { Non- } \\
\text { sensitizer }\end{array}$ & & & & & REACH (2008-2014) & \\
\hline 2-Methyloxolane & CC1CCCO1 & & $\begin{array}{l}\text { Non- } \\
\text { sensitizer }\end{array}$ & & & & & REACH (2008-2014) & \\
\hline 2-Nitroaniline & $\operatorname{Nc} 1 \operatorname{cccc} 1 \mathrm{~N}(=0)=0$ & & $\begin{array}{l}\text { Non- } \\
\text { sensitizer }\end{array}$ & & & & & REACH (2008-2014) & \\
\hline $\begin{array}{l}2-[(5,5-d i m e t h y l-2- \\
\text { sulfanylidene-1,3-dioxa- } \\
2 \$ \mid \wedge\{5\}- \\
\text { phosphacyclohex-2- } \\
\text { yl)oxy]-5,5-dimethyl-2- } \\
\text { sulfanylidene-1,3-dioxa- } \\
\left.2 \$\right|^{\wedge}\{5\}- \\
\text { phosphacyclohexane }\end{array}$ & $\begin{array}{l}\mathrm{CC} 1(\mathrm{C}) \mathrm{COP}(=\mathrm{S})(\mathrm{OP} 2(=\mathrm{S} \\
\operatorname{OOCC}(\mathrm{C})(\mathrm{C}) \mathrm{CO} 2) \mathrm{OC} 1\end{array}$ & & $\begin{array}{l}\text { Non- } \\
\text { sensitizer }\end{array}$ & & & & & REACH (2008-2014) & \\
\hline $\begin{array}{l}\text { 2-[(E)-2-(4- } \\
\text { methylamino-2- } \\
\text { sulfonatophenyl)etheny } \\
\text { I]-5-[[4-morpholin-4-yl- } \\
6 \text {-[(4- } \\
\text { sulfonatophenyl)amino] } \\
\text {-1,3,5-triazin-2- } \\
\text { yl]amino]benzenesulfo }\end{array}$ & $\begin{array}{l}\text { OS }(=0)(=0) c 1 c c c(N c 2 n \\
c(N c 3 c c c(C=C c 4 c c c(N c \\
5 n c(N c 6 c c c(c c 6) S(O)(= \\
O)=0) n c(n 5) N 5 c c O C C \\
5) \operatorname{cc} 4 S(O)(=0)=0) c(c 3) \\
S(O)(=0)=O) n c(n 2) N 2 C \\
\operatorname{cOCC} 2) c c 1\end{array}$ & & $\begin{array}{l}\text { Non- } \\
\text { sensitizer }\end{array}$ & & & & & REACH (2008-2014) & \\
\hline
\end{tabular}




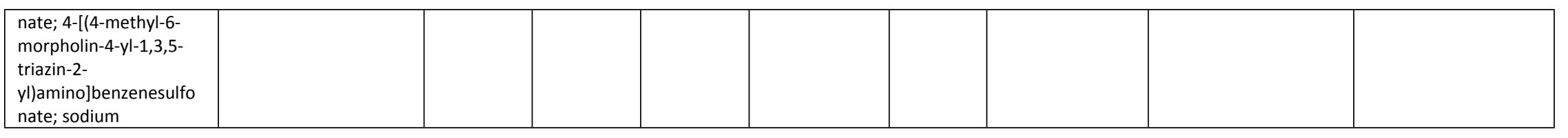

\begin{tabular}{|c|c|c|c|c|c|c|c|c|c|}
\hline Compound name & SMILES & $\begin{array}{l}\text { Human } \\
\text { result }\end{array}$ & $\begin{array}{l}\text { LLNA } \\
\text { result }\end{array}$ & DPRA & KeratinoSens & h-CLAT & $\begin{array}{l}\text { Human } \\
\text { reference }\end{array}$ & LLNA reference & Non-animal reference \\
\hline $\begin{array}{l}\text { 2-[(E)-2-cyano-3-(4- } \\
\text { diethylaminophenyl)pr } \\
\text { op-2-enoyl]oxyethyl-[3- } \\
\text { [(E)-2-cyano-3-(4- } \\
\text { diethylaminophenyl)pr } \\
\text { op-2-enoyl]oxypropyl]- } \\
\text { dimethylazanium } \\
\text { chloride }\end{array}$ & $\begin{array}{l}\mathrm{CCN}(\mathrm{CC}) \mathrm{c} 1 \mathrm{ccc}(\mathrm{C}=\mathrm{C}(\mathrm{C \#} \\
\mathrm{N}) \mathrm{C}(=\mathrm{O}) \mathrm{OCCC}[\mathrm{N}+](\mathrm{C})(\mathrm{C} \\
) \mathrm{CCOC}(=\mathrm{O}) \mathrm{C}(=\mathrm{Cc} 2 \mathrm{ccc}(\mathrm{c} \\
\mathrm{c} 2) \mathrm{N}(\mathrm{CC}) \mathrm{CC}) \mathrm{C \# N}) \mathrm{cc} 1\end{array}$ & & $\begin{array}{l}\text { Non- } \\
\text { sensitizer }\end{array}$ & & & & & REACH (2008-2014) & \\
\hline $\begin{array}{l}\text { 2-[(benzyloxy)- } \\
\text { imino]malonic acid }\end{array}$ & $\begin{array}{l}O C(=O) C(=N O C c 1 c c c c c \\
1) C(O)=O\end{array}$ & & $\begin{array}{l}\text { Non- } \\
\text { sensitizer }\end{array}$ & & & & & ICCVAM (2013) & \\
\hline $\begin{array}{l}\text { 2-[1,3-dioxo-1-[(2-oxo- } \\
\text { 1,3- } \\
\text { dihydrobenzimidazol-5- } \\
\text { yl)amino]butan-2- } \\
\text { yl]diazenylbenzoic acid }\end{array}$ & $\begin{array}{l}\mathrm{CC}(=0) \mathrm{C}(=\mathrm{NN} 1 \mathrm{ccccc} 1 \\
\mathrm{C}(\mathrm{O})=\mathrm{O}) \mathrm{C}(=\mathrm{O}) \mathrm{Nc} 1 \mathrm{ccc} 2 \\
\mathrm{NC}(=\mathrm{O}) \mathrm{Nc} 2 \mathrm{c} 1\end{array}$ & & $\begin{array}{l}\text { Non- } \\
\text { sensitizer }\end{array}$ & & & & & REACH (2008-2014) & \\
\hline $\begin{array}{l}\text { 2-[1-(1- } \\
\text { methoxypropan-2- } \\
\text { yloxy)propan-2- } \\
\text { yloxy]propan-1-ol }\end{array}$ & $\begin{array}{l}\operatorname{COCC}(\mathrm{C}) \mathrm{OCC}(\mathrm{C}) \mathrm{OC}(\mathrm{C}) \mathrm{C} \\
\mathrm{O}\end{array}$ & & $\begin{array}{l}\text { Non- } \\
\text { sensitizer }\end{array}$ & & & & & REACH (2008-2014) & \\
\hline $\begin{array}{l}\text { 2-[2,2- } \\
\text { bis(hydroxymethyl)buto } \\
\text { xymethyl]-2- } \\
\text { ethylpropane-1,3-diol }\end{array}$ & $\begin{array}{l}\mathrm{CCC}(\mathrm{CO})(\mathrm{CO}) \mathrm{COCC}(\mathrm{CC}) \\
(\mathrm{CO}) \mathrm{CO}\end{array}$ & & $\begin{array}{l}\text { Non- } \\
\text { sensitizer }\end{array}$ & & & & & REACH (2008-2014) & \\
\hline $\begin{array}{l}\text { 2-[2,5-dichloro-4-[2,5- } \\
\text { dichloro-4-[1-[(2,4- } \\
\text { dimethylphenyl)amino] } \\
-1,3-\text { dioxobutan-2- } \\
\text { yl]diazenylphenyl]phen } \\
\text { yl]diazenyl-N-(2,4- } \\
\text { dimethylphenyl)-3- } \\
\text { oxobutanamide }\end{array}$ & $\begin{array}{l}\mathrm{CC}(=\mathrm{O}) \mathrm{C}(=\mathrm{NNc} 1 \mathrm{cc}(\mathrm{Cl}) \mathrm{cl} \\
\mathrm{cc} 1 \mathrm{Cl})- \\
\mathrm{c1}(\mathrm{cc}(\mathrm{Cl}) \mathrm{c}(\mathrm{NN}=\mathrm{C}(\mathrm{C}(\mathrm{C})=\mathrm{O} \\
) \mathrm{C}=\mathrm{O}) \mathrm{Nc} 2 \mathrm{ccc}(\mathrm{C}) \mathrm{cc} 2 \mathrm{C}) \mathrm{c} \\
\mathrm{c} 1 \mathrm{Cl}) \mathrm{C}(=\mathrm{O}) \mathrm{Nc} 1 \mathrm{ccc}(\mathrm{C}) \mathrm{cc} \\
1 \mathrm{C}\end{array}$ & & $\begin{array}{l}\text { Non- } \\
\text { sensitizer }\end{array}$ & & & & & REACH (2008-2014) & \\
\hline $\begin{array}{l}\text { 2-[2-(2- } \\
\text { ethoxyethoxy)ethoxy]et } \\
\text { hyl 2-methylprop-2-- } \\
\text { enoate }\end{array}$ & $\begin{array}{l}\mathrm{CCOCCOC \operatorname {coc } O C}(=0) \\
\mathrm{C}(\mathrm{C})=\mathrm{C}\end{array}$ & & $\begin{array}{l}\text { Non- } \\
\text { sensitizer }\end{array}$ & & & & & REACH (2008-2014) & \\
\hline $\begin{array}{l}\text { 2-[2-(2-methoxy-5- } \\
\text { nitrophenyl)hydrazin-1- } \\
\text { ylidene]-N-(2- }\end{array}$ & $\begin{array}{l}\operatorname{COc} 1 \operatorname{ccccc} 1 N C(=0) C(= \\
\operatorname{NNc1cc}(\operatorname{ccc} 10 C) N(=0) \\
=0) C(C)=0\end{array}$ & & $\begin{array}{l}\text { Non- } \\
\text { sensitizer }\end{array}$ & & & & & REACH (2008-2014) & \\
\hline
\end{tabular}




\begin{tabular}{|l|l|l|l|l|l|l|l|}
\hline $\begin{array}{l}\text { methoxyphenyl)-3- } \\
\text { oxobutanamide }\end{array}$ & & & & & & & \\
\hline $\begin{array}{l}2-[2- \\
\text { (phenoxy)ethoxy]ethan } \\
\text { ol }\end{array}$ & OCCOCCOc1ccccc1 & & $\begin{array}{l}\text { Non- } \\
\text { sensitizer }\end{array}$ & & & & \\
\hline
\end{tabular}

\begin{tabular}{|c|c|c|c|c|c|c|c|c|c|}
\hline Compound name & SMILES & $\begin{array}{l}\text { Human } \\
\text { result }\end{array}$ & $\begin{array}{l}\text { LLNA } \\
\text { result }\end{array}$ & DPRA & KeratinoSens & h-CLAT & $\begin{array}{l}\text { Human } \\
\text { reference }\end{array}$ & LLNA reference & Non-animal reference \\
\hline $\begin{array}{l}\text { 2-[2-[2-(2- } \\
\text { ethylhexanoyloxy)ethox } \\
\text { y]ethoxy]ethyl 2- } \\
\text { ethylhexanoate }\end{array}$ & $\begin{array}{l}\mathrm{CCCCC}(\mathrm{CC}) \mathrm{C}(=0) \mathrm{OCCO} \\
\mathrm{CCOCCOC}(=0) \mathrm{C}(\mathrm{CC}) \mathrm{CC} \\
\mathrm{CC}\end{array}$ & & $\begin{array}{l}\text { Non- } \\
\text { sensitizer }\end{array}$ & & & & & REACH (2008-2014) & \\
\hline $\begin{array}{l}\text { 2-[2-[2-[2-[1,3-dioxo-1- } \\
\text { [(2-oxo-1,3- } \\
\text { dihydrobenzimidazol-5- } \\
\text { yl)amino]butan-2- } \\
\text { yl]diazenylphenoxy]eth } \\
\text { oxy]phenyl]diazenyl-3- } \\
\text { oxo-N-(2-oxo-1,3- } \\
\text { dihydrobenzimidazol-5- } \\
\text { yl)butanamide }\end{array}$ & $\begin{array}{l}\mathrm{CC}(=0) \mathrm{C}(=\mathrm{NN} \operatorname{Nc} 1 \mathrm{ccccc} 1 \\
\mathrm{OCCO} 1 \mathrm{ccccc} 1 \mathrm{NN}=\mathrm{C}(\mathrm{C} \\
(C)=0) \mathrm{C}(=0) \mathrm{Nc} 1 \mathrm{ccc} 2 \mathrm{~N} \\
\mathrm{C}(=0) \mathrm{Nc} 2 \mathrm{c} 1) \mathrm{C}(=\mathrm{O}) \mathrm{Nc1} \\
\operatorname{ccc} 2 \mathrm{NC}(=0) \mathrm{Nc} 2 \mathrm{c1}\end{array}$ & & $\begin{array}{l}\text { Non- } \\
\text { sensitizer }\end{array}$ & & & & & REACH (2008-2014) & \\
\hline $\begin{array}{l}\text { 2-[2-chloro-4-[3-chloro- } \\
\text { 4-[1,3-dioxo-1- } \\
\text { (phenylamino)butan-2- } \\
\text { yl]diazenylphenyl]phen } \\
\text { yl]diazenyl-3-oxo- } \mathrm{N} \text { - } \\
\text { phenylbutanamide }\end{array}$ & 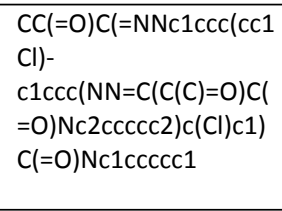 & & $\begin{array}{l}\text { Non- } \\
\text { sensitizer }\end{array}$ & & & & & REACH (2008-2014) & \\
\hline $\begin{array}{l}\text { 2-[2-chloro-4-[3-chloro- } \\
4-[1-[(2,4- \\
\text { dimethylphenyl)amino] } \\
\text {-1,3-dioxobutan-2- } \\
\text { yl]diazenylphenyl]phen } \\
\text { yl]diazenyl-N-(2,4- } \\
\text { dimethylphenyl)-3- } \\
\text { oxobutanamide }\end{array}$ & $\begin{array}{l}\mathrm{CC}(=0) \mathrm{C}(=\mathrm{NN} \operatorname{cocc}(\mathrm{cc} 1 \\
\mathrm{Cl})- \\
\mathrm{c1} 1 \mathrm{ccc}(\mathrm{NN}=\mathrm{C}(\mathrm{C}(\mathrm{C})=\mathrm{O}) \mathrm{Cl} \\
=0) \mathrm{Nc} 2 \mathrm{ccc}(\mathrm{C}) \mathrm{cc} 2 \mathrm{C}) \mathrm{c}(\mathrm{Cl} \\
) \mathrm{c} 1) \mathrm{C}(=\mathrm{O}) \mathrm{Nc} 1 \mathrm{ccc}(\mathrm{C}) \mathrm{cc} 1 \\
\mathrm{C}\end{array}$ & & $\begin{array}{l}\text { Non- } \\
\text { sensitizer }\end{array}$ & & & & & REACH (2008-2014) & \\
\hline $\begin{array}{l}\text { 2-[2-chloro-4-[3-chloro- } \\
\text { 4-[1-[(2- } \\
\text { methoxyphenyl)amino]- } \\
\text { 1,3-dioxobutan-2- } \\
\text { yl]diazenylphenyl]phen } \\
\text { yl]diazenyl-N-(2- } \\
\text { methoxyphenyl)-3- } \\
\text { oxobutanamide }\end{array}$ & $\begin{array}{l}\operatorname{COc1} 1 \operatorname{ccccc} 1 N C(=0) C(= \\
N N c 1 \operatorname{ccc}(\operatorname{cc} 1 \mathrm{Cl})- \\
\operatorname{c1ccc}(\mathrm{NN}=\mathrm{C}(\mathrm{C}(\mathrm{C})=0) \mathrm{C}( \\
=0) \operatorname{Nc} 2 \operatorname{ccccc} 2 \mathrm{OC}) \mathrm{c}(\mathrm{Cl}) \mathrm{c} \\
1) \mathrm{C}(\mathrm{C})=0\end{array}$ & & $\begin{array}{l}\text { Non- } \\
\text { sensitizer }\end{array}$ & & & & & REACH (2008-2014) & \\
\hline $\begin{array}{l}\text { 2-[2-chloro-4-[3-chloro- } \\
\text { 4-[1-[(2- } \\
\text { methylphenyl)amino]- }\end{array}$ & $\begin{array}{l}\mathrm{CC}(=0) \mathrm{C}(=\mathrm{NN} 1 \mathrm{ccc}(\mathrm{cc} 1 \\
\mathrm{Cl})- \\
\mathrm{c1} \operatorname{ccc}(\mathrm{NN}=\mathrm{C}(\mathrm{C}(\mathrm{C})=\mathrm{O}) \mathrm{Cl}(\end{array}$ & & $\begin{array}{l}\text { Non- } \\
\text { sensitizer }\end{array}$ & & & & & REACH (2008-2014) & \\
\hline
\end{tabular}




\begin{tabular}{|l|l|l|l|l|l|l|l|}
\hline $\begin{array}{l}1,3-\text { dioxobutan-2- } \\
\text { yl]diazenylphenyl]phen } \\
\text { yl]diazenyl-N-(2- } \\
\text { methylphenyl)-3- } \\
\text { oxobutanamide }\end{array}$ & $\begin{array}{l}=0) \text { Nc2cccc2C)(Cl)c1 } \\
\text { C(=O)Nc1ccccc1C }\end{array}$ & & & & & & \\
\hline
\end{tabular}

\begin{tabular}{|c|c|c|c|c|c|c|c|c|c|}
\hline Compound name & SMILES & $\begin{array}{l}\text { Human } \\
\text { result }\end{array}$ & $\begin{array}{l}\text { LLNA } \\
\text { result }\end{array}$ & DPRA & KeratinoSens & h-CLAT & $\begin{array}{l}\text { Human } \\
\text { reference }\end{array}$ & LLNA reference & Non-animal reference \\
\hline $\begin{array}{l}\text { 2-[2-chloro-4-[3-chloro- } \\
\text { 4-[1-[(4-chloro-2,5- } \\
\text { dimethoxyphenyl)amin } \\
\text { o]-1,3-dioxobutan-2- } \\
\text { yl]diazenylphenyl]phen } \\
\text { yl]diazenyl-N-(4-chloro- } \\
\text { 2,5-dimethoxyphenyl)- } \\
\text { 3-oxobutanamide }\end{array}$ & $\begin{array}{l}\operatorname{COc1cc}(N C(=O) C(=N N c \\
2 \operatorname{ccc}(\operatorname{cc} 2 \mathrm{Cl})- \\
\mathrm{c} 2 \mathrm{ccc}(\mathrm{NN}=\mathrm{C}(\mathrm{C}(\mathrm{C})=\mathrm{O}) \mathrm{Cl} \\
=\mathrm{O}) \mathrm{Nc} 3 \mathrm{cc}(\mathrm{OC}) \mathrm{c}(\mathrm{Cl}) \mathrm{cc} 3 \\
\mathrm{OC}) \mathrm{c}(\mathrm{Cl}) \mathrm{c} 2) \mathrm{C}(\mathrm{C})=\mathrm{O}) \mathrm{c}(\mathrm{O} \\
\text { C) } \mathrm{cc1Cl}\end{array}$ & & $\begin{array}{l}\text { Non- } \\
\text { sensitizer }\end{array}$ & & & & & REACH (2008-2014) & \\
\hline $\begin{array}{l}\text { 2-[2-chloro-4-[3-chloro- } \\
\text { 4-[1-[(4- } \\
\text { ethoxyphenyl)amino]- } \\
\text { 1,3-dioxobutan-2- } \\
\text { yl]diazenylphenyl]phen } \\
\text { yl]diazenyl-N-(4- } \\
\text { ethoxyphenyl)-3- } \\
\text { oxobutanamide }\end{array}$ & $\begin{array}{l}\mathrm{CCOc1} 1 \mathrm{ccc}(\mathrm{NC}(=\mathrm{O}) \mathrm{C}(=\mathrm{N} \\
\mathrm{Nc} 2 \operatorname{ccc}(\mathrm{cc} 2 \mathrm{Cl})- \\
\mathrm{c} 2 \mathrm{ccc}(\mathrm{NN}=\mathrm{C}(\mathrm{C}(\mathrm{C})=\mathrm{O}) \mathrm{C}( \\
=0) \mathrm{Nc} 3 \operatorname{ccc}(\mathrm{OCC}) \mathrm{cc} 3) \mathrm{c}( \\
\mathrm{Cl}) \mathrm{c} 2) \mathrm{C}(\mathrm{C})=0) \mathrm{cc} 1\end{array}$ & & $\begin{array}{l}\text { Non- } \\
\text { sensitizer }\end{array}$ & & & & & REACH (2008-2014) & \\
\hline $\begin{array}{l}\text { 2-[2-chloro-4-[3-chloro- } \\
\text { 4-[1-[(4- } \\
\text { methylphenyl)amino]- } \\
\text { 1,3-dioxobutan-2- } \\
\text { yl]diazenylphenyl]phen } \\
\text { yl]diazenyl-N-(4- } \\
\text { methylphenyl)-3- } \\
\text { oxobutanamide }\end{array}$ & $\begin{array}{l}\mathrm{CC}(=0) \mathrm{C}(=\mathrm{NN} \operatorname{cocc}(\mathrm{cc} 1 \\
\mathrm{Cl})- \\
\mathrm{c1} 1 \mathrm{ccc}(\mathrm{NN}=\mathrm{C}(\mathrm{C}(\mathrm{C})=\mathrm{O}) \mathrm{Cl} \\
=0) \mathrm{Nc} 2 \mathrm{ccc}(\mathrm{C}) \mathrm{cc} 2) \mathrm{c}(\mathrm{Cl}) \mathrm{c} \\
1) \mathrm{C}(=0) \mathrm{Nc} 1 \mathrm{ccc}(\mathrm{C}) \mathrm{cc} 1\end{array}$ & & $\begin{array}{l}\text { Non- } \\
\text { sensitizer }\end{array}$ & & & & & REACH (2008-2014) & \\
\hline $\begin{array}{l}\text { 2-[2-ethoxy-5-(4- } \\
\text { ethylpiperazin-1- } \\
\text { yl)sulfonylphenyl]-5- } \\
\text { methyl-7-propyl-1H- } \\
\text { imidazo[5,1- } \\
\text { f][1,2,4]triazin-4-one }\end{array}$ & $\begin{array}{l}\mathrm{CCCc} 1 \mathrm{nc}(\mathrm{C}) \mathrm{c} 2 \mathrm{C}(=\mathrm{O}) \mathrm{N}= \\
\mathrm{C}(\mathrm{Nn} 12) \mathrm{c} 1 \mathrm{cc}(\operatorname{ccc} 1 \mathrm{OCC}) \\
\mathrm{S}(=\mathrm{O})(=\mathrm{O}) \mathrm{N} 1 \mathrm{CCN}(\mathrm{CC}) \mathrm{C} \\
\mathrm{C} 1\end{array}$ & & $\begin{array}{l}\text { Non- } \\
\text { sensitizer }\end{array}$ & & & & & REACH (2008-2014) & \\
\hline $\begin{array}{l}\text { 2-[3,3-bis(3-tert-butyl- } \\
\text { 4- } \\
\text { hydroxyphenyl)butanoy } \\
\text { loxy]ethyl 3,3-bis(3- } \\
\text { tert-butyl-4- } \\
\text { hydroxyphenyl)butanoa } \\
\text { te }\end{array}$ & $\begin{array}{l}\mathrm{CC}(\mathrm{C})(\mathrm{C}) \mathrm{c} 1 \mathrm{cc}(\mathrm{ccc} 10) \mathrm{C}( \\
\mathrm{C})(\mathrm{CC}(=\mathrm{O}) \mathrm{OCCOC}(=\mathrm{O}) \mathrm{C} \\
\mathrm{C}(\mathrm{C})(\mathrm{c} 1 \mathrm{ccc}(\mathrm{O}) \mathrm{c}(\mathrm{c} 1) \mathrm{C}(\mathrm{C})( \\
\mathrm{C}) \mathrm{C}) \mathrm{c} 1 \mathrm{ccc}(\mathrm{O}) \mathrm{c}(\mathrm{c} 1) \mathrm{C}(\mathrm{C})( \\
\mathrm{C)C}) \mathrm{c} 1 \mathrm{ccc}(\mathrm{O}) \mathrm{c}(\mathrm{c} 1) \mathrm{C}(\mathrm{C})( \\
\mathrm{C}) \mathrm{C}\end{array}$ & & $\begin{array}{l}\text { Non- } \\
\text { sensitizer }\end{array}$ & & & & & REACH (2008-2014) & \\
\hline
\end{tabular}




\begin{tabular}{|l|l|l|l|l|l|l|l|}
\hline $\begin{array}{l}\text { 2-[4-[2-[4-(2- } \\
\text { hydroxyethoxy)phenyl] } \\
\text { propan-2- } \\
\text { yl]phenoxy]ethanol }\end{array}$ & $\begin{array}{l}\mathrm{CC}(\mathrm{C})(\mathrm{c} 1 \mathrm{ccc}(\mathrm{OCCO}) \mathrm{cc} 1) \\
\mathrm{c1ccc}(\mathrm{OCCO}) \mathrm{cc} 1\end{array}$ & $\begin{array}{l}\text { Non- } \\
\text { sensitizer }\end{array}$ & & & & & \\
\hline
\end{tabular}

\begin{tabular}{|c|c|c|c|c|c|c|c|c|c|}
\hline Compound name & SMILES & $\begin{array}{l}\text { Human } \\
\text { result }\end{array}$ & $\begin{array}{l}\text { LLNA } \\
\text { result }\end{array}$ & DPRA & KeratinoSens & h-CLAT & $\begin{array}{l}\text { Human } \\
\text { reference }\end{array}$ & LLNA reference & Non-animal reference \\
\hline $\begin{array}{l}\text { 2-[[3-hydroxy-2,2- } \\
\text { bis(hydroxymethyl)prop } \\
\text { oxy]methyl]-2- } \\
\text { (hydroxymethyl)propan } \\
\text { e-1,3-diol }\end{array}$ & $\begin{array}{l}\mathrm{OCC}(\mathrm{CO})(\mathrm{CO}) \mathrm{COCC}(\mathrm{CO}) \\
(\mathrm{CO}) \mathrm{CO}\end{array}$ & & $\begin{array}{l}\text { Non- } \\
\text { sensitizer }\end{array}$ & & & & & REACH (2008-2014) & \\
\hline $\begin{array}{l}\text { 2-[[4-[bis[4- } \\
\text { (phenylamino)phenyl]m } \\
\text { ethylidene]-1- } \\
\text { cyclohexa-2,5- } \\
\text { dienylidene]amino]ben } \\
\text { zenesulfonic acid } \\
\end{array}$ & $\begin{array}{l}\mathrm{OS}(=\mathrm{O})(=\mathrm{O}) \mathrm{c} 1 \mathrm{ccccc} 1 \mathrm{~N}= \\
\mathrm{C} 1 \mathrm{C}=\mathrm{CC}(\mathrm{C}=\mathrm{C} 1)=\mathrm{C}(\mathrm{c} 1 \mathrm{ccc} \\
(\mathrm{Nc} 2 \mathrm{ccccc} 2) \mathrm{cc} 1) \mathrm{c} 1 \mathrm{ccc}( \\
\mathrm{Nc} 2 \mathrm{ccccc} 2) \mathrm{cc} 1\end{array}$ & & $\begin{array}{l}\text { Non- } \\
\text { sensitizer }\end{array}$ & & & & & REACH (2008-2014) & \\
\hline $\begin{array}{l}\text { 2-amino-3-(1H-indol-3- } \\
\text { yl)propanoic acid }\end{array}$ & $\begin{array}{l}\mathrm{NC}(\mathrm{Cc} 1 \mathrm{c}[\mathrm{nH}] \mathrm{c} 2 \mathrm{ccccc} 12 \\
\mathrm{C}(\mathrm{O})=\mathrm{O}\end{array}$ & & $\begin{array}{l}\text { Non- } \\
\text { sensitizer }\end{array}$ & & & & & REACH (2008-2014) & \\
\hline $\begin{array}{l}\text { 2-amino-4,5- } \\
\text { dichlorobenzenesulfoni } \\
\text { c acid }\end{array}$ & $\begin{array}{l}\mathrm{Nc} 1 \mathrm{cc}(\mathrm{Cl}) \mathrm{c}(\mathrm{Cl}) \mathrm{cc} 1 \mathrm{~S}(\mathrm{O})( \\
=0)=0\end{array}$ & & $\begin{array}{l}\text { Non- } \\
\text { sensitizer }\end{array}$ & & & & & REACH (2008-2014) & \\
\hline $\begin{array}{l}\text { 2-ethyl-2- } \\
\text { (hydroxymethyl)propan } \\
\text { e-1,3-diol }\end{array}$ & $\mathrm{CCC}(\mathrm{CO})(\mathrm{CO}) \mathrm{CO}$ & & $\begin{array}{l}\text { Non- } \\
\text { sensitizer }\end{array}$ & & & & & REACH (2008-2014) & \\
\hline $\begin{array}{l}\text { 2-ethyl-N,N-bis(2- } \\
\text { ethylhexyl)hexan-1- } \\
\text { amine }\end{array}$ & $\begin{array}{l}\mathrm{CCCCC}(\mathrm{CC}) \mathrm{CN}(\mathrm{CC}(\mathrm{CC}) \mathrm{C} \\
\mathrm{CCC}) \mathrm{CC}(\mathrm{CC}) \mathrm{CCCC}\end{array}$ & & $\begin{array}{l}\text { Non- } \\
\text { sensitizer }\end{array}$ & & & & & REACH (2008-2014) & \\
\hline $\begin{array}{l}\text { 2-ethylanthracene- } \\
\text { 9,10-dione }\end{array}$ & $\begin{array}{l}\mathrm{cCc} 1 \mathrm{ccc} 2 \mathrm{C}(=0) \mathrm{c} 3 \mathrm{ccccc} \\
3 \mathrm{C}(=0) \mathrm{c} 2 \mathrm{c} 1\end{array}$ & & $\begin{array}{l}\text { Non- } \\
\text { sensitizer }\end{array}$ & & & & & REACH (2008-2014) & \\
\hline 2-ethylsulfanylethanol & CCSCCO & & $\begin{array}{l}\text { Non- } \\
\text { sensitizer }\end{array}$ & & & & & REACH (2008-2014) & \\
\hline $\begin{array}{l}\text { 2-fluoro-5- } \\
\text { methanesulfonylbenzoi } \\
\text { c acid }\end{array}$ & $\begin{array}{l}\mathrm{CS}(=0)(=0) \operatorname{c} 1 \mathrm{ccc}(\mathrm{F}) \mathrm{c}(\mathrm{c} \\
1) \mathrm{C}(0)=0\end{array}$ & & $\begin{array}{l}\text { Non- } \\
\text { sensitizer }\end{array}$ & & & & & REACH (2008-2014) & \\
\hline $\begin{array}{l}\text { 2-hydroxy-1-(4-\{[4-(2- } \\
\text { hydroxy-2- } \\
\text { methylpropanoyl)pheny } \\
\text { I]methyl\}phenyl)-2- } \\
\text { methylpropan-1-one }\end{array}$ & $\begin{array}{l}\mathrm{CC}(\mathrm{C})(\mathrm{O}) \mathrm{C}(=0) \mathrm{c} 1 \mathrm{ccc}(\mathrm{Cc} \\
2 \mathrm{ccc}(\mathrm{cc} 2) \mathrm{C}(=0) \mathrm{C}(\mathrm{C})(\mathrm{C}) \\
0) \mathrm{cc} 1\end{array}$ & & $\begin{array}{l}\text { Non- } \\
\text { sensitizer }\end{array}$ & & & & & REACH (2008-2014) & \\
\hline 2-methyl-1H-imidazole & Cc1ncc[nH]1 & & $\begin{array}{l}\text { Non- } \\
\text { sensitizer }\end{array}$ & & & & & REACH (2008-2014) & \\
\hline
\end{tabular}




\begin{tabular}{|c|c|c|c|c|c|c|c|c|c|}
\hline $\begin{array}{l}\text { 2-methyl-4- } \\
\text { phenylpentan-1-ol }\end{array}$ & $\mathrm{CC}(\mathrm{CO}) \mathrm{CC}(\mathrm{C}) \mathrm{c} 1 \mathrm{ccccc} 1$ & & $\begin{array}{l}\text { Non- } \\
\text { sensitizer }\end{array}$ & & & & & REACH (2008-2014) & \\
\hline $\begin{array}{l}\text { 2-methyl-6- } \\
\text { (pentyloxy)oxane-3,4,5- } \\
\text { triol }\end{array}$ & $\begin{array}{l}\text { CCCCCOC1OC(C)C(O)C( } \\
\text { o) C10 }\end{array}$ & & $\begin{array}{l}\text { Non- } \\
\text { sensitizer }\end{array}$ & & & & & REACH (2008-2014) & \\
\hline $\begin{array}{l}\text { 2-methyl-N-propan-2- } \\
\text { ylprop-2-enamide }\end{array}$ & $C C(C) N C(=O) C(C)=C$ & & $\begin{array}{l}\text { Non- } \\
\text { sensitizer }\end{array}$ & & & & & REACH (2008-2014) & \\
\hline $\begin{array}{l}\text { 2-methylprop-2- } \\
\text { enamide }\end{array}$ & $\mathrm{CC}(=\mathrm{C}) \mathrm{C}(\mathrm{N})=\mathrm{O}$ & & $\begin{array}{l}\text { Non- } \\
\text { sensitizer }\end{array}$ & & & & & REACH (2008-2014) & \\
\hline Compound name & SMILES & $\begin{array}{l}\text { Human } \\
\text { result }\end{array}$ & $\begin{array}{l}\text { LLNA } \\
\text { result }\end{array}$ & DPRA & KeratinoSens & h-CLAT & $\begin{array}{l}\text { Human } \\
\text { reference }\end{array}$ & LLNA reference & Non-animal reference \\
\hline $\begin{array}{l}\text { 2-morpholin-4- } \\
\text { ylethanol }\end{array}$ & OCCN1CCOCC1 & & $\begin{array}{l}\text { Non- } \\
\text { sensitizer }\end{array}$ & & & & & REACH (2008-2014) & \\
\hline $\begin{array}{l}\text { 2-naphthalen-2- } \\
\text { yloxyethanol }\end{array}$ & OCCOc1ccc2ccccc2c1 & & $\begin{array}{l}\text { Non- } \\
\text { sensitizer }\end{array}$ & & & & & REACH (2008-2014) & \\
\hline $\begin{array}{l}\text { 2-oxo-N-[4-[[4-[(2- } \\
\text { oxoazepane-1- } \\
\text { carbonyl)amino]phenyl] } \\
\text { methyl]phenyl]azepane } \\
\text {-1-carboxamide }\end{array}$ & $\begin{array}{l}\mathrm{O}=\mathrm{C}(\mathrm{Nc} 1 \mathrm{ccc}(\mathrm{Cc} 2 \mathrm{ccc}(\mathrm{NC} \\
(=\mathrm{O}) \mathrm{N} 3 \mathrm{CCCCCC} 3=\mathrm{O}) \mathrm{cc} \\
2) \mathrm{cc} 1) \mathrm{N} 1 \mathrm{CCCCCC} 1=\mathrm{O}\end{array}$ & & $\begin{array}{l}\text { Non- } \\
\text { sensitizer }\end{array}$ & & & & & REACH (2008-2014) & \\
\hline $\begin{array}{l}\text { 2-propan-2-yloxyethyl } \\
\text { acetate }\end{array}$ & $\mathrm{CC}(\mathrm{C}) \mathrm{OCCOC}(\mathrm{C})=\mathrm{O}$ & & $\begin{array}{l}\text { Non- } \\
\text { sensitizer }\end{array}$ & & & & & REACH (2008-2014) & \\
\hline $\begin{array}{l}\text { 2-propan-2- } \\
\text { yloxypropane }\end{array}$ & $\mathrm{CC}(\mathrm{C}) \mathrm{OC}(\mathrm{C}) \mathrm{C}$ & & $\begin{array}{l}\text { Non- } \\
\text { sensitizer }\end{array}$ & & & & & REACH (2008-2014) & \\
\hline $\begin{array}{l}\text { 2-tert-butyl-4-[1-(5- } \\
\text { tert-butyl-4-hydroxy-2- } \\
\text { methylphenyl)butyl]-5- } \\
\text { methylphenol }\end{array}$ & $\begin{array}{l}\mathrm{CCCC}(\mathrm{c} 1 \mathrm{cc}(\mathrm{c}(\mathrm{O}) \mathrm{cc} 1 \mathrm{C}) \mathrm{Cl} \\
\mathrm{C})(\mathrm{C}) \mathrm{C}) \mathrm{c1cc}(\mathrm{c}(\mathrm{O}) \mathrm{cc1C}) \mathrm{C} \\
\text { (C)(C)C }\end{array}$ & & $\begin{array}{l}\text { Non- } \\
\text { sensitizer }\end{array}$ & & & & & REACH (2008-2014) & \\
\hline $\begin{array}{l}\text { 2-\{2-[3,3'-dichloro-4'- } \\
\text { (2-\{1-[(2,4- } \\
\text { dimethylphenyl)carbam } \\
\text { oyl]-2- } \\
\text { oxopropylidene\}hydrazi } \\
\text { n-1-yl)-[1,1'-biphenyl]- } \\
\text { 4-yl]hydrazin-1- } \\
\text { ylidene\}-N-(2- } \\
\text { methoxyphenyl)-3- } \\
\text { oxobutanamide }\end{array}$ & $\begin{array}{l}\operatorname{COc1ccccc} 1 N C(=0) C(= \\
N N \operatorname{ccc}(\operatorname{cc} 1 \mathrm{Cl})- \\
\mathrm{c} 1 \operatorname{ccc}(\mathrm{NN}=\mathrm{C}(\mathrm{C}(\mathrm{C})=\mathrm{O}) \mathrm{C}( \\
=0) \operatorname{Nc} 2 \operatorname{ccc}(\mathrm{C}) \mathrm{cc} 2 \mathrm{C}) \mathrm{c}(\mathrm{Cl} \\
\operatorname{c1}) \mathrm{C}(\mathrm{C})=0\end{array}$ & & $\begin{array}{l}\text { Non- } \\
\text { sensitizer }\end{array}$ & & & & & REACH (2008-2014) & \\
\hline $\begin{array}{l}\text { 2-\{[2,8-dimethyl-2- } \\
(4,8,12- \\
\text { trimethyltridecyl)-3,4- } \\
\text { dihydro-2H-1- } \\
\text { benzopyran-6-yl]oxy\}-6- } \\
\text { (hydroxymethyl)oxane- } \\
\text { 3,4,5-triol }\end{array}$ & $\begin{array}{l}\mathrm{CC}(\mathrm{C}) \mathrm{CCCC}(\mathrm{C}) \mathrm{CCCC}(\mathrm{C}) \mathrm{C} \\
\mathrm{CCC} 1(\mathrm{C}) \mathrm{CCc} 2 \mathrm{cc}(\mathrm{OC} 3 \mathrm{OC} \\
(\mathrm{CO}) \mathrm{C}(\mathrm{O}) \mathrm{C}(\mathrm{O}) \mathrm{C} 3 \mathrm{O}) \mathrm{cc}(\mathrm{C}) \\
\mathrm{c} 2 \mathrm{O} 1\end{array}$ & & $\begin{array}{l}\text { Non- } \\
\text { sensitizer }\end{array}$ & & & & & REACH (2008-2014) & \\
\hline
\end{tabular}




\begin{tabular}{|c|c|c|c|c|c|c|c|c|c|}
\hline Compound name & SMILES & $\begin{array}{l}\text { Human } \\
\text { result }\end{array}$ & $\begin{array}{l}\text { LLNA } \\
\text { result }\end{array}$ & DPRA & KeratinoSens & h-CLAT & $\begin{array}{l}\text { Human } \\
\text { reference }\end{array}$ & LLNA reference & Non-animal reference \\
\hline $\begin{array}{l}\text { 3'-[(2z)-[1-(3,4- } \\
\text { dimethylphenyl)-1,5- } \\
\text { dihydro-3-methyl)-5- } \\
\text { oxo-4h-pyrazol-4- } \\
\text { ylidene]hydrazino]-2'- } \\
\text { hydroxy-[1,1'-biphenyl]- } \\
\text { 3-carboxylic acid, } \\
\text { compound with 2- } \\
\text { aminoethanol (2:1) }\end{array}$ & $\begin{array}{l}\mathrm{CC} 1=\mathrm{C}(\mathrm{N}=\mathrm{Nc} 2 \mathrm{cccc}(- \\
\mathrm{c} 3 \operatorname{cccc}(\mathrm{c} 3) \mathrm{C}(\mathrm{O})=\mathrm{O}) \mathrm{c} 2 \mathrm{O}) \\
\mathrm{C}(=\mathrm{O}) \mathrm{N}(\mathrm{N} 1) \mathrm{c} 1 \mathrm{ccc}(\mathrm{C}) \mathrm{c}(\mathrm{C} \\
) \mathrm{c} 1\end{array}$ & & $\begin{array}{l}\text { Non- } \\
\text { sensitizer }\end{array}$ & & & & & ICCVAM (2013) & \\
\hline $\begin{array}{l}3,3,4,4,5,5,6,6,7,7,8,8,8 \\
\text {-tridecafluorooctyl 2- } \\
\text { methylprop-2-enoate }\end{array}$ & $\begin{array}{l}\mathrm{CC}(=\mathrm{C}) \mathrm{C}(=0) O C C C(\mathrm{~F})(\mathrm{F}) \\
\mathrm{C}(\mathrm{F})(\mathrm{F}) \mathrm{C}(\mathrm{F})(\mathrm{F}) \mathrm{C}(\mathrm{F})(\mathrm{F}) \mathrm{C}(\mathrm{F} \\
)(\mathrm{F}) \mathrm{C}(\mathrm{F})(\mathrm{F}) \mathrm{F}\end{array}$ & & $\begin{array}{l}\text { Non- } \\
\text { sensitizer }\end{array}$ & & & & & REACH (2008-2014) & \\
\hline $\begin{array}{l}3,3,4,4,5,5,6,6,7,7,8,8,8 \\
\text {-tridecafluorooctyl } \\
\text { prop-2-enoate }\end{array}$ & $\begin{array}{l}F C(F)(F) C(F)(F) C(F)(F) C( \\
F)(F) C(F)(F) C(F)(F) C C O \\
C(=O) C=C\end{array}$ & & $\begin{array}{l}\text { Non- } \\
\text { sensitizer }\end{array}$ & & & & & REACH (2008-2014) & \\
\hline $\begin{array}{l}\text { 3,3-bis(4- } \\
\text { hydroxyphenyl)-2- } \\
\text { benzofuran-1-one }\end{array}$ & $\begin{array}{l}\text { Oc1ccc }(\operatorname{cc} 1) \operatorname{c1}(\mathrm{OC}(=0) \\
\mathrm{c} 2 \operatorname{ccccc} 12) \operatorname{c1} 1 \mathrm{ccc}(\mathrm{O}) \mathrm{cc} 1\end{array}$ & & $\begin{array}{l}\text { Non- } \\
\text { sensitizer }\end{array}$ & & & & & REACH (2008-2014) & \\
\hline $\begin{array}{l}\text { 3,4,5,6-tetrahydroxy-2- } \\
{[(3,4,5,6 \text {-tetrahydroxy- }} \\
\text { 1-oxohexan-2- } \\
\text { yl)oxy]hexanal }\end{array}$ & $\begin{array}{l}\mathrm{OCC}(\mathrm{O}) \mathrm{C}(\mathrm{O}) \mathrm{C}(\mathrm{O}) \mathrm{C}(\mathrm{OCC} \\
\mathrm{C}=0) \mathrm{C}(\mathrm{O}) \mathrm{C}(\mathrm{O}) \mathrm{C}(\mathrm{O}) \mathrm{CO}) \\
\mathrm{C}=\mathrm{O}\end{array}$ & & $\begin{array}{l}\text { Non- } \\
\text { sensitizer }\end{array}$ & & & & & REACH (2008-2014) & \\
\hline $\begin{array}{l}\text { 3,9-bis(1-cyclohex-3- } \\
\text { enyl)-2,4,8,10- } \\
\text { tetraoxaspiro[5.5]unde } \\
\text { cane }\end{array}$ & $\begin{array}{l}\mathrm{C} 1 \mathrm{CC}(\mathrm{CC}=\mathrm{C} 1) \mathrm{C} 10 \mathrm{CC} 2( \\
\mathrm{CO} 1) \mathrm{COC}(\mathrm{OC} 2) \mathrm{C} 1 \mathrm{CCC}= \\
\mathrm{CC} 1\end{array}$ & & $\begin{array}{l}\text { Non- } \\
\text { sensitizer }\end{array}$ & & & & & REACH (2008-2014) & \\
\hline $\begin{array}{l}\text { 3,9-dioctadecoxy- } \\
\text { 2,4,8,10-tetraoxa-3,9- } \\
\text { diphosphaspiro[5.5]und } \\
\text { ecane }\end{array}$ & $\begin{array}{l}\text { CCCCCCCCCCCCCCCCC } \\
\text { COP1OCC2(CO1)COP( } \\
\text { OCCCCCCCCCCCCCCCC } \\
\text { CC)OC2 }\end{array}$ & & $\begin{array}{l}\text { Non- } \\
\text { sensitizer }\end{array}$ & & & & & REACH (2008-2014) & \\
\hline $\begin{array}{l}\text { 3-(2H-Benzotriazol-2- } \\
\text { yl)-5-sec-butyl-4- } \\
\text { hydroxybenzenesulfoni } \\
\text { c acid }\end{array}$ & $\begin{array}{l}\mathrm{CCC}(\mathrm{C}) \mathrm{c} 1 \mathrm{cc}(\mathrm{cc}(\mathrm{c} 10)- \\
\mathrm{n} 1 \mathrm{nc} 2 \operatorname{ccccc} 2 \mathrm{n} 1) \mathrm{S}(0)(= \\
0)=0\end{array}$ & & $\begin{array}{l}\text { Non- } \\
\text { sensitizer }\end{array}$ & & & & & REACH (2008-2014) & \\
\hline
\end{tabular}




\begin{tabular}{|c|c|c|c|c|c|c|c|c|c|}
\hline $\begin{array}{l}\text { 3-(3,5-di-tert-butyl-4- } \\
\text { hydroxyphenyl)-N- } \\
\text { octadecylpropanamide }\end{array}$ & $\begin{array}{l}\text { CCCCCCCCCCCCCCCCCC } \\
\mathrm{CNC}(=0) \mathrm{CCC} 1 \mathrm{cc}(\mathrm{c}(\mathrm{O}) \mathrm{c}( \\
\mathrm{c} 1) \mathrm{C}(\mathrm{C})(\mathrm{C}) \mathrm{C}) \mathrm{C}(\mathrm{C})(\mathrm{C}) \mathrm{C}\end{array}$ & & $\begin{array}{l}\text { Non- } \\
\text { sensitizer }\end{array}$ & & & & & REACH (2008-2014) & \\
\hline $\begin{array}{l}\text { 3-(5-methyl-2-propan- } \\
2- \\
\text { ylcyclohexyl)oxypropan } \\
\text { e-1,2-diol }\end{array}$ & $\begin{array}{l}\mathrm{CC}(\mathrm{C}) \mathrm{C} 1 \mathrm{CCC}(\mathrm{C}) \mathrm{CC} 10 \mathrm{CC} \\
\text { (O)CO }\end{array}$ & & $\begin{array}{l}\text { Non- } \\
\text { sensitizer }\end{array}$ & & & & & REACH (2008-2014) & \\
\hline $\begin{array}{l}\text { 3- } \\
\text { Aminobenzenesulfonic } \\
\text { acid }\end{array}$ & $\begin{array}{l}\operatorname{Nc1} 1 \mathrm{cccc}(\mathrm{c} 1) \mathrm{S}(\mathrm{O})(=0)= \\
\mathrm{O}\end{array}$ & & $\begin{array}{l}\text { Non- } \\
\text { sensitizer }\end{array}$ & & & & & REACH (2008-2014) & \\
\hline Compound name & SMILES & $\begin{array}{l}\text { Human } \\
\text { result }\end{array}$ & $\begin{array}{l}\text { LLNA } \\
\text { result }\end{array}$ & DPRA & KeratinoSens & h-CLAT & $\begin{array}{l}\text { Human } \\
\text { reference }\end{array}$ & LLNA reference & Non-animal reference \\
\hline $\begin{array}{l}\text { 3-Hydroxy-2,4,7,14- } \\
\text { tetramethyl-9-oxo-4- } \\
\text { vinyltricyclo[5.4.3.01,8] } \\
\text { tetradec-6-yl glycolate }\end{array}$ & $\begin{array}{l}\mathrm{CC} 1 \mathrm{CCC} 23 \mathrm{CCC}(=)) \mathrm{C2C} \\
1(\mathrm{C}) \mathrm{C}(\mathrm{CC}(\mathrm{C})(\mathrm{C}=\mathrm{C}) \mathrm{C}(\mathrm{O}) \mathrm{C} \\
3 \mathrm{C}) \mathrm{OC}(=0) \mathrm{CO}\end{array}$ & & $\begin{array}{l}\text { Non- } \\
\text { sensitizer }\end{array}$ & & & & & ICCVAM (2013) & \\
\hline $\begin{array}{l}\text { 3-Hydroxy-2- } \\
\text { nitropyridine }\end{array}$ & Oc1cconc1N $(=0)=0$ & & $\begin{array}{l}\text { Non- } \\
\text { sensitizer }\end{array}$ & & & & & ICCVAM (2013) & \\
\hline $\begin{array}{l}\text { 3-Hydroxy-2-phenyl-4- } \\
\text { quinolinecarboxylic acid }\end{array}$ & $\begin{array}{l}\text { OC(=0)c1c(O)c(nc2ccc } \\
\text { cc12)-c1ccccc1 }\end{array}$ & & $\begin{array}{l}\text { Non- } \\
\text { sensitizer }\end{array}$ & & & & & ICCVAM (2013) & \\
\hline $\begin{array}{l}\text { 3-Hydroxy-4- } \\
\text { methoxybenzaldehyde }\end{array}$ & $\operatorname{coc} 1 \operatorname{ccc}(\mathrm{C}=0) \operatorname{cc} 10$ & & $\begin{array}{l}\text { Non- } \\
\text { sensitizer }\end{array}$ & & & & & ICCVAM (2013) & \\
\hline $\begin{array}{l}\text { 3- } \\
\text { Hydroxypropanenitrile }\end{array}$ & OCCC\#N & & $\begin{array}{l}\text { Non- } \\
\text { sensitizer }\end{array}$ & & & & & REACH (2008-2014) & \\
\hline 3-Methyl-1-butanol & $\mathrm{CC}(\mathrm{C}) \mathrm{CCO}$ & & $\begin{array}{l}\text { Non- } \\
\text { sensitizer }\end{array}$ & & & & & $\begin{array}{l}\text { ICCVAM }(2013)+ \\
\operatorname{REACH}(2008-2014)\end{array}$ & \\
\hline 3-Propoxybenzoic acid & $\operatorname{ccCOc} 1 \operatorname{ccc}(\mathrm{c} 1) \mathrm{C}(0)=0$ & & $\begin{array}{l}\text { Non- } \\
\text { sensitizer }\end{array}$ & & & & & ICCVAM (2013) & \\
\hline $\begin{array}{l}\text { 3-[(2R)-3-[[2-(2,3- } \\
\text { Dihydro-1H-inden-2-yl)- } \\
\text { 1,1-dimethyl- } \\
\text { ethyl]amino]-2- } \\
\text { hydroxypropoxy]-4,5- } \\
\text { difluoro-benzene } \\
\text { propanoic acid }\end{array}$ & $\begin{array}{l}\mathrm{CC}(\mathrm{C})(\mathrm{CC} 1 \mathrm{Cc} 2 \mathrm{ccccc} 2 \mathrm{C} 1 \\
) \mathrm{NCC}(\mathrm{O}) \mathrm{CO} 1 \mathrm{cc}(\mathrm{CCC}(\mathrm{O} \\
)=0) \mathrm{cc}(\mathrm{F}) \mathrm{c} 1 \mathrm{~F}\end{array}$ & & $\begin{array}{l}\text { Non- } \\
\text { sensitizer }\end{array}$ & & & & & ICCVAM (2013) & \\
\hline $\begin{array}{l}\text { 3-[3,5-bis(3- } \\
\text { dimethylaminopropyl)- } \\
\text { 1,3,5-triazinan-1-yl]- } \\
\text { N,N-dimethylpropan-1- } \\
\text { amine }\end{array}$ & $\begin{array}{l}\mathrm{CN}(\mathrm{C}) \mathrm{CCCN} 1 \mathrm{CN}(\mathrm{CCCN}( \\
\mathrm{C}) \mathrm{C}) \mathrm{CN}(\mathrm{CCCN}(\mathrm{C}) \mathrm{C}) \mathrm{C} 1\end{array}$ & & $\begin{array}{l}\text { Non- } \\
\text { sensitizer }\end{array}$ & & & & & REACH (2008-2014) & \\
\hline $\begin{array}{l}\text { 3-[4-[(6- } \\
\text { bromohexyl)oxy]- } \\
\text { butyl]benzene- } \\
\text { sulfonamide } \\
\end{array}$ & $\begin{array}{l}\mathrm{NS}(=0)(=0) \mathrm{c} 1 \operatorname{cccc}(\mathrm{CCC} \\
\operatorname{cOCCCCCCBr}) \mathrm{c} 1\end{array}$ & & $\begin{array}{l}\text { Non- } \\
\text { sensitizer }\end{array}$ & & & & & ICCVAM (2013) & \\
\hline $\begin{array}{l}\text { 3-amino-1- } \\
\text { methylthiourea }\end{array}$ & $\mathrm{CNC}(=\mathrm{S}) \mathrm{NN}$ & & $\begin{array}{l}\text { Non- } \\
\text { sensitizer }\end{array}$ & & & & & REACH (2008-2014) & \\
\hline
\end{tabular}




\begin{tabular}{|c|c|c|c|c|c|c|c|c|c|}
\hline $\begin{array}{l}\text { 3-aminoprop-2-ynoic } \\
\text { acid }\end{array}$ & $\mathrm{NCCC}(\mathrm{O})=\mathrm{O}$ & & $\begin{array}{l}\text { Non- } \\
\text { sensitizer }\end{array}$ & & & & & REACH (2008-2014) & \\
\hline $\begin{array}{l}\text { 3-benzyl-1-\{6- } \\
\text { [(benzylcarbamoyl)ami } \\
\text { no]hexyl\}urea }\end{array}$ & $\begin{array}{l}\mathrm{O}=\mathrm{C}(\mathrm{NCCCCCCNC}(=\mathrm{O}) \\
\mathrm{NCC} 1 \mathrm{ccccc} 1) \mathrm{NCc} 1 \mathrm{ccccc} \\
1\end{array}$ & & $\begin{array}{l}\text { Non- } \\
\text { sensitizer }\end{array}$ & & & & & REACH (2008-2014) & \\
\hline $\begin{array}{l}\text { 3-bromo-2,2- } \\
\text { bis(bromomethyl)propa } \\
\text { n-1-ol }\end{array}$ & $\mathrm{OCC}(\mathrm{CBr})(\mathrm{CBr}) \mathrm{CBr}$ & & $\begin{array}{l}\text { Non- } \\
\text { sensitizer }\end{array}$ & & & & & REACH (2008-2014) & \\
\hline $\begin{array}{l}\text { 3-hydroxy-2- } \\
\text { (hydroxymethyl)-2- } \\
\text { methylpropanal }\end{array}$ & $\mathrm{CC}(\mathrm{CO})(\mathrm{CO}) \mathrm{C}=\mathrm{O}$ & & $\begin{array}{l}\text { Non- } \\
\text { sensitizer }\end{array}$ & & & & & REACH (2008-2014) & \\
\hline Compound name & SMILES & $\begin{array}{l}\text { Human } \\
\text { result }\end{array}$ & $\begin{array}{l}\text { LLNA } \\
\text { result }\end{array}$ & DPRA & KeratinoSens & h-CLAT & $\begin{array}{l}\text { Human } \\
\text { reference }\end{array}$ & LLNA reference & Non-animal reference \\
\hline $\begin{array}{l}\text { 3-hydroxy-4-[2-(4- } \\
\text { methyl-2- } \\
\text { sulfophenyl)diazen-1- } \\
\text { yl]naphthalene-2- } \\
\text { carboxylic acid }\end{array}$ & $\begin{array}{l}\operatorname{Cc} 1 \mathrm{ccc}(\mathrm{N}=\mathrm{Nc} 2 \mathrm{c}(\mathrm{O}) \mathrm{c}(\mathrm{cc} \\
3 \operatorname{ccccc} 23) \mathrm{C}(\mathrm{O})=\mathrm{O}) \mathrm{c}(\mathrm{c} 1) \\
\mathrm{S}(\mathrm{O})(=\mathrm{O})=\mathrm{O}\end{array}$ & & $\begin{array}{l}\text { Non- } \\
\text { sensitizer }\end{array}$ & & & & & REACH (2008-2014) & \\
\hline 3-hydroxynonyl acetate & $\begin{array}{l}\operatorname{CCCCCCC}(0) \operatorname{CcOC}(C)= \\
0\end{array}$ & & $\begin{array}{l}\text { Non- } \\
\text { sensitizer }\end{array}$ & & & & & REACH (2008-2014) & \\
\hline 3-methyl-2H-pyrazole & $\mathrm{Cc} 1 \mathrm{ccn}[\mathrm{nH}] 1$ & & $\begin{array}{l}\text { Non- } \\
\text { sensitizer }\end{array}$ & & & & & REACH (2008-2014) & \\
\hline $\begin{array}{l}\text { 3-methyl-4-\{4-[4-(prop- } \\
\text { 2- } \\
\text { enoyloxy)butoxy]benzo } \\
\text { yloxy\}phenyl 4-[4- } \\
\text { (prop-2- } \\
\text { enoyloxy)butoxy]benzo } \\
\text { ate }\end{array}$ & $\begin{array}{l}\mathrm{Cc} 1 \mathrm{cc}(\mathrm{OC}(=)) \mathrm{c} 2 \mathrm{ccc}(\mathrm{OC} \\
\mathrm{cccOC}(=\mathrm{O}) \mathrm{C}=\mathrm{C}) \mathrm{cc} 2) \mathrm{ccc} \\
1 \mathrm{OC}(=\mathrm{O}) \mathrm{c} 1 \mathrm{ccc}(\mathrm{OCCCC} \\
\mathrm{OC}(=\mathrm{O}) \mathrm{C}=\mathrm{C}) \mathrm{cc} 1\end{array}$ & & $\begin{array}{l}\text { Non- } \\
\text { sensitizer }\end{array}$ & & & & & REACH (2008-2014) & \\
\hline $\begin{array}{l}\text { 3-octanoyloxypropyl } \\
\text { octanoate }\end{array}$ & $\begin{array}{l}\text { CCCCCCCCC }(=0) \text { OCCCO } \\
\mathrm{C}(=0) \operatorname{ccccccc}\end{array}$ & & $\begin{array}{l}\text { Non- } \\
\text { sensitizer }\end{array}$ & & & & & REACH (2008-2014) & \\
\hline $\begin{array}{l}\text { 3-oxo-N-(2-oxo-1,3- } \\
\text { dihydrobenzimidazol-5- } \\
\text { yl)-2-[2- } \\
\text { (trifluoromethyl)phenyl } \\
\text { ]diazenylbutanamide }\end{array}$ & $\begin{array}{l}\mathrm{CC}(=\mathrm{O}) \mathrm{C}(=\mathrm{NN} \operatorname{Nc} 1 \mathrm{ccccc} 1 \\
\mathrm{C}(\mathrm{F})(\mathrm{F}) \mathrm{F}) \mathrm{C}(=\mathrm{O}) \mathrm{Nc1} \mathrm{ccc} 2 \\
\mathrm{NC}(=\mathrm{O}) \mathrm{Nc} 2 \mathrm{c} 1\end{array}$ & & $\begin{array}{l}\text { Non- } \\
\text { sensitizer }\end{array}$ & & & & & REACH (2008-2014) & \\
\hline $\begin{array}{l}\text { 4'-(Trifluoro-methyl)- } \\
{\left[1,1^{1} \text {-biphenyl]-4- }\right.} \\
\text { carboxaldehyde }\end{array}$ & $\begin{array}{l}\mathrm{FC}(\mathrm{F})(\mathrm{F}) \mathrm{c} 1 \mathrm{ccc}(\mathrm{cc} 1)- \\
\mathrm{c} 1 \mathrm{ccc}(\mathrm{C}=0) \mathrm{cc} 1\end{array}$ & & $\begin{array}{l}\text { Non- } \\
\text { sensitizer }\end{array}$ & & & & & ICCVAM (2013) & \\
\hline $\begin{array}{l}\text { 4,4'- } \\
\text { bis(methoxymethyl)- } \\
\text { 1,1'-biphenyl }\end{array}$ & $\begin{array}{l}\operatorname{cOCc} 1 \operatorname{ccc}(\mathrm{cc} 1)- \\
\operatorname{c1ccc}(\operatorname{COC}) \operatorname{cc} 1\end{array}$ & & $\begin{array}{l}\text { Non- } \\
\text { sensitizer }\end{array}$ & & & & & REACH (2008-2014) & \\
\hline $\begin{array}{l}\text { 4-(1- } \\
\text { phenylethyl)benzene- } \\
\text { 1,3-diol }\end{array}$ & $\begin{array}{l}\mathrm{CC}(\mathrm{c} 1 \mathrm{ccccc} 1) \mathrm{c} 1 \mathrm{ccc}(0) \mathrm{c} \\
\mathrm{c} 10\end{array}$ & & $\begin{array}{l}\text { Non- } \\
\text { sensitizer }\end{array}$ & & & & & REACH (2008-2014) & \\
\hline
\end{tabular}




\begin{tabular}{|c|c|c|c|c|c|c|c|c|c|}
\hline $\begin{array}{l}\text { 4-(4- } \\
\text { aminophenyl)sulfonylan } \\
\text { iline }\end{array}$ & $\begin{array}{l}\operatorname{Nc1} 1 \operatorname{ccc}(\operatorname{cc} 1) S(=0)(=0) c \\
1 \operatorname{ccc}(N) \operatorname{cc} 1\end{array}$ & & $\begin{array}{l}\text { Non- } \\
\text { sensitizer }\end{array}$ & & & & & REACH (2008-2014) & \\
\hline $\begin{array}{l}\text { 4-(4- } \\
\text { hydroxyphenyl)sulfonyl } \\
\text { phenol }\end{array}$ & $\begin{array}{l}\operatorname{Oc} 1 \operatorname{ccc}(\operatorname{cc} 1) S(=0)(=0) c \\
1 \operatorname{ccc}(0) \operatorname{cc} 1\end{array}$ & & $\begin{array}{l}\text { Non- } \\
\text { sensitizer }\end{array}$ & & & & & REACH (2008-2014) & \\
\hline $\begin{array}{l}\text { 4-(decanoyloxy)benzoic } \\
\text { acid }\end{array}$ & $\begin{array}{l}\text { CCCCCCCCCC(=0)Oc1c } \\
\operatorname{cc}(\operatorname{cc} 1) \mathrm{C}(\mathrm{O})=\mathrm{O}\end{array}$ & & $\begin{array}{l}\text { Non- } \\
\text { sensitizer }\end{array}$ & & & & & REACH (2008-2014) & \\
\hline $\begin{array}{l}\text { 4-Fluoro-2-pyrrolidine- } \\
\text { carboxamide }\end{array}$ & $\mathrm{NC}(=0) \mathrm{C} 1 \mathrm{CC}(\mathrm{F}) \mathrm{CN} 1$ & & $\begin{array}{l}\text { Non- } \\
\text { sensitizer }\end{array}$ & & & & & ICCVAM (2013) & \\
\hline $\begin{array}{l}\text { 4-Isopropyl-1- } \\
\text { methylenecyclohexane }\end{array}$ & $\mathrm{CC}(\mathrm{C}) \mathrm{C} 1 \mathrm{CCC}(=\mathrm{C}) \mathrm{CC} 1$ & & $\begin{array}{l}\text { Non- } \\
\text { sensitizer }\end{array}$ & & & & & ICCVAM (2013) & \\
\hline Compound name & SMILES & $\begin{array}{l}\text { Human } \\
\text { result }\end{array}$ & $\begin{array}{l}\text { LLNA } \\
\text { result }\end{array}$ & DPRA & KeratinoSens & h-CLAT & $\begin{array}{l}\text { Human } \\
\text { reference }\end{array}$ & LLNA reference & Non-animal reference \\
\hline $\begin{array}{l}\text { 4- } \\
\text { Methylbenzenesulfona } \\
\text { mide }\end{array}$ & $\operatorname{Cc} 1 \operatorname{ccc}(\operatorname{cc} 1) S(N)(=0)=0$ & & $\begin{array}{l}\text { Non- } \\
\text { sensitizer }\end{array}$ & & & & & REACH (2008-2014) & \\
\hline 4-Phenylbutan-2-one & $\mathrm{CC}(=0) \operatorname{CCc} 1 \mathrm{ccccc} 1$ & & $\begin{array}{l}\text { Non- } \\
\text { sensitizer }\end{array}$ & & & & & REACH (2008-2014) & \\
\hline $\begin{array}{l}\text { 4-[(4-amino-3- } \\
\text { chlorophenyl)methyl]- } \\
\text { 2-chloroaniline }\end{array}$ & $\begin{array}{l}\mathrm{Nc} 1 \mathrm{ccc}(\mathrm{Cc} 2 \operatorname{ccc}(\mathrm{N}) \mathrm{c}(\mathrm{Cl}) \mathrm{c} \\
2) \operatorname{cc} 1 \mathrm{Cl}\end{array}$ & & $\begin{array}{l}\text { Non- } \\
\text { sensitizer }\end{array}$ & & & & & REACH (2008-2014) & \\
\hline $\begin{array}{l}\text { 4-[2-(4- } \\
\text { hydroxycyclohexyl)prop } \\
\text { an-2-yl]cyclohexan-1-ol }\end{array}$ & $\begin{array}{l}\mathrm{CC}(\mathrm{C})(\mathrm{C} 1 \mathrm{CCC}(\mathrm{O}) \mathrm{CC} 1) \mathrm{C} 1 \\
\mathrm{CCC}(\mathrm{O}) \mathrm{CC} 1\end{array}$ & & $\begin{array}{l}\text { Non- } \\
\text { sensitizer }\end{array}$ & & & & & REACH (2008-2014) & \\
\hline $\begin{array}{l}\text { 4-[2-chloro-4-[3-chloro- } \\
\text { 4-[[3-methyl-1-(4- } \\
\text { methylphenyl)-5-oxo- } \\
\text { 4H-pyrazol-4- } \\
\text { yl]diazenyl]phenyl]phen } \\
\text { yl]diazenyl-5-methyl-2- } \\
\text { (4-methylphenyl)-4H- } \\
\text { pyrazol-3-one }\end{array}$ & $\begin{array}{l}\mathrm{CC1}=\mathrm{NN}(\mathrm{C}(=\mathrm{O}) \mathrm{C} 1 \mathrm{~N}=\mathrm{Nc} \\
1 \mathrm{ccc}(\mathrm{cc} 1 \mathrm{Cl})- \\
\mathrm{c} 1 \mathrm{ccc}(\mathrm{N}=\mathrm{NC} 2 \mathrm{C}(\mathrm{C})=\mathrm{NN}( \\
\mathrm{C} 2=\mathrm{O}) \mathrm{c} 2 \mathrm{ccc}(\mathrm{C}) \mathrm{cc} 2) \mathrm{c}(\mathrm{Cl}) \\
\mathrm{c} 1) \mathrm{c} 1 \mathrm{ccc}(\mathrm{C}) \mathrm{cc} 1\end{array}$ & & $\begin{array}{l}\text { Non- } \\
\text { sensitizer }\end{array}$ & & & & & REACH (2008-2014) & \\
\hline $\begin{array}{l}\text { 4-[4-[[(3R)-1-butyl-3- } \\
{[(\mathrm{R}) \text {-cyclohexyl- }} \\
\text { hydroxymethyl]-2,5- } \\
\text { dioxo-1,4,9- } \\
\text { triazaspiro[5.5]-undec- } \\
\text { 9- } \\
\text { yl]methyl]phenoxy]ben } \\
\text { zoic acid } \\
\end{array}$ & $\begin{array}{l}\mathrm{CCCCN} 1 \mathrm{C}(=\mathrm{O}) \mathrm{C}(\mathrm{NC}(=\mathrm{O}) \\
\mathrm{C} 11 \mathrm{CCN}(\mathrm{Cc} 2 \mathrm{ccc}(\mathrm{Oc} 3 \mathrm{cc} \\
\mathrm{c}(\mathrm{cc} 3) \mathrm{C}(\mathrm{O})=0) \mathrm{cc} 2) \mathrm{CC} 1) \\
\mathrm{C}(\mathrm{O}) \mathrm{C} 1 \mathrm{CCCCC} 1\end{array}$ & & $\begin{array}{l}\text { Non- } \\
\text { sensitizer }\end{array}$ & & & & & ICCVAM (2013) & \\
\hline $\begin{array}{l}\text { 4-[4-[[4-chloro-3- } \\
\text { (trifluoromethyl)phenyl } \\
\text { ]carbamoylamino]phen } \\
\text { oxy]-N-methylpyridine- } \\
\text { 2-carboxamide }\end{array}$ & $\begin{array}{l}\mathrm{CNC}(=0) \operatorname{c1cc}(\mathrm{Oc} 2 \mathrm{ccc}(\mathrm{N} \\
\mathrm{C}(=\mathrm{O}) \mathrm{Nc} 3 \operatorname{ccc}(\mathrm{Cl}) \mathrm{c}(\mathrm{c} 3) \mathrm{C}( \\
\mathrm{F})(\mathrm{F}) \mathrm{F}) \mathrm{cc} 2) \mathrm{ccn} 1\end{array}$ & & $\begin{array}{l}\text { Non- } \\
\text { sensitizer }\end{array}$ & & & & & REACH (2008-2014) & \\
\hline
\end{tabular}




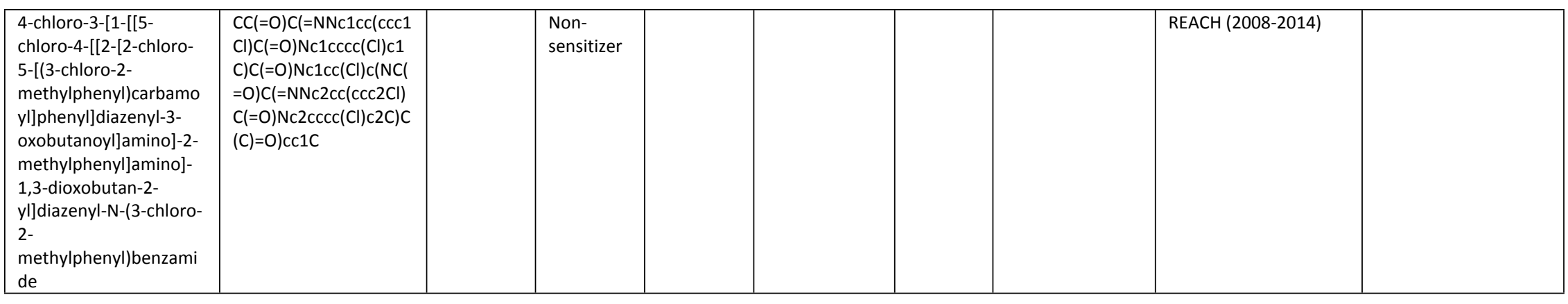

\begin{tabular}{|c|c|c|c|c|c|c|c|c|c|}
\hline Compound name & SMILES & $\begin{array}{l}\text { Human } \\
\text { result }\end{array}$ & $\begin{array}{l}\text { LLNA } \\
\text { result }\end{array}$ & DPRA & KeratinoSens & h-CLAT & $\begin{array}{l}\text { Human } \\
\text { reference }\end{array}$ & LLNA reference & Non-animal reference \\
\hline 4-ethenoxybutan-1-ol & $\mathrm{OCCCCOC}=\mathrm{C}$ & & $\begin{array}{l}\text { Non- } \\
\text { sensitizer }\end{array}$ & & & & & REACH (2008-2014) & \\
\hline $\begin{array}{l}\text { 4-fluoro-4-methyl-2- } \\
\text { [(2,2,2-trifluoro-1-\{4'- } \\
\text { methanesulfonyl-[1,1'- } \\
\text { biphenyl]-4- } \\
\text { yl\}ethyl)amino]pentano } \\
\text { ic acid }\end{array}$ & $\begin{array}{l}C C(C)(F) C C(N C(c 1 c c c(c \\
c 1)- \\
c 1 c c c(c c 1) S(C)(=0)=0) \\
C(F)(F) F) C(O)=0\end{array}$ & & $\begin{array}{l}\text { Non- } \\
\text { sensitizer }\end{array}$ & & & & & REACH (2008-2014) & \\
\hline $\begin{array}{l}\text { 4-methyl-1,3- } \\
\text { dihydrobenzimidazole- } \\
\text { 2-thione }\end{array}$ & Cc1cccc2NC(=S)Nc12 & & $\begin{array}{l}\text { Non- } \\
\text { sensitizer }\end{array}$ & & & & & REACH (2008-2014) & \\
\hline $\begin{array}{l}\text { 4-methyl-2-propyloxan- } \\
\text { 4-yl acetate }\end{array}$ & $\begin{array}{l}\mathrm{CCCC1CC}(\mathrm{C})(\mathrm{CCO} 1) \mathrm{OC}( \\
\mathrm{C})=\mathrm{O}\end{array}$ & & $\begin{array}{l}\text { Non- } \\
\text { sensitizer }\end{array}$ & & & & & REACH (2008-2014) & \\
\hline $\begin{array}{l}\text { 4S-1-(tert- } \\
\text { Butoxycarbonyl)-4- } \\
\text { fluoro-L-prolinamide }\end{array}$ & $\begin{array}{l}\mathrm{CC}(\mathrm{C})(\mathrm{C}) \mathrm{OC}(=\mathrm{O}) \mathrm{N} 1 \mathrm{CC}(\mathrm{F} \\
\operatorname{CC1C}(\mathrm{N})=0\end{array}$ & & $\begin{array}{l}\text { Non- } \\
\text { sensitizer }\end{array}$ & & & & & ICCVAM (2013) & \\
\hline $\begin{array}{l}\text { 5,5,11'a-trimethyl- } \\
\text { 3',3'a,3'b,4',5',8',9',9'a,9 } \\
\text { 'b,10',11',11'a- } \\
\text { dodecahydro-2'H- } \\
\text { dispiro[1,3-dioxane- } \\
\text { 2,1'- } \\
\text { cyclopenta[a]phenanthr } \\
\text { ene-7',2"- } \\
\text { [1,3]dithiolan]-10'-one }\end{array}$ & $\begin{array}{l}\text { CC12CC(=O)C3C(CCC4= } \\
\text { CC5 (CCC34)SCCS5)C1C } \\
\text { CC21OCC(C)(C)CO1 }\end{array}$ & & $\begin{array}{l}\text { Non- } \\
\text { sensitizer }\end{array}$ & & & & & REACH (2008-2014) & \\
\hline $\begin{array}{l}\text { 5-Amino-2- } \\
\text { methylbenzene- } \\
\text { sulfonamide }\end{array}$ & $\begin{array}{l}\operatorname{Cc} 1 \operatorname{ccc}(\mathrm{N}) \operatorname{cc} 1 \mathrm{~S}(\mathrm{~N})(=\mathrm{O})= \\
\mathrm{O}\end{array}$ & & $\begin{array}{l}\text { Non- } \\
\text { sensitizer }\end{array}$ & & & & & ICCVAM (2013) & \\
\hline $\begin{array}{l}\text { 5-Chloro-2,6- } \\
\text { dimethoxy-4- } \\
\text { methylquinoline }\end{array}$ & $\begin{array}{l}\operatorname{cOc} 1 \mathrm{cc}(\mathrm{C}) \mathrm{c} 2 \mathrm{c}(\mathrm{Cl}) \mathrm{c}(\mathrm{OC}) \\
\operatorname{ccc} 2 \mathrm{n} 1\end{array}$ & & $\begin{array}{l}\text { Non- } \\
\text { sensitizer }\end{array}$ & & & & & ICCVAM (2013) & \\
\hline
\end{tabular}




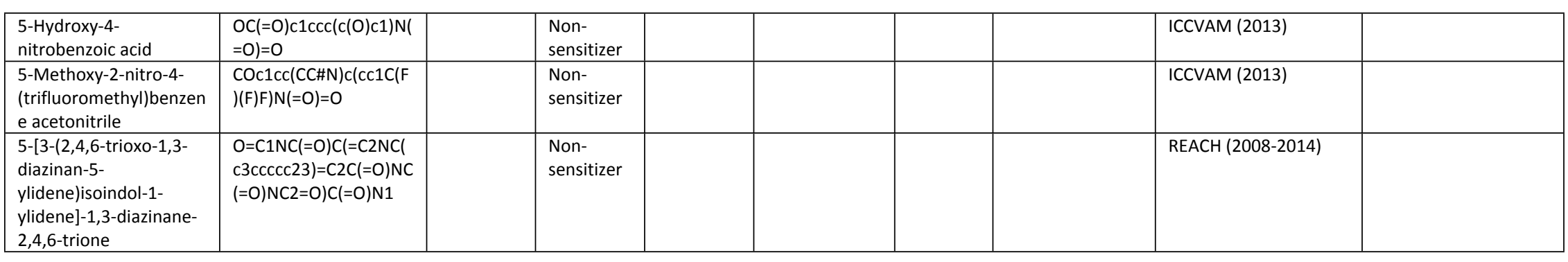

\begin{tabular}{|c|c|c|c|c|c|c|c|c|c|}
\hline Compound name & SMILES & $\begin{array}{l}\text { Human } \\
\text { result }\end{array}$ & $\begin{array}{l}\text { LLNA } \\
\text { result }\end{array}$ & DPRA & KeratinoSens & h-CLAT & $\begin{array}{l}\text { Human } \\
\text { reference }\end{array}$ & LLNA reference & Non-animal reference \\
\hline $\begin{array}{l}\text { 5-[[4-[(2,3-dimethyl-2h- } \\
\text { indazol-6-yl)- } \\
\text { methylamino]-2- } \\
\text { pyrimidinyl]amino]-2- } \\
\text { methylbenzene- } \\
\text { sulfonamide }\end{array}$ & $\begin{array}{l}\mathrm{CN}(\operatorname{c1ccc} 2 \mathrm{c}(\mathrm{C}) n(\mathrm{C}) n \mathrm{nc} c \\
1) \mathrm{c} 1 \mathrm{ccnc}(\mathrm{Nc} 2 \operatorname{ccc}(\mathrm{C}) \mathrm{c}(\mathrm{c} \\
2) \mathrm{S}(\mathrm{N})(=\mathrm{O})=0) n 1\end{array}$ & & $\begin{array}{l}\text { Non- } \\
\text { sensitizer }\end{array}$ & & & & & ICCVAM (2013) & \\
\hline $\begin{array}{l}\text { 5-amino-3- } \\
\text { methylthiophene-2,4- } \\
\text { dicarbonitrile }\end{array}$ & Cc1c(sc(N)c1C\#N)C\#N & & $\begin{array}{l}\text { Non- } \\
\text { sensitizer }\end{array}$ & & & & & REACH (2008-2014) & \\
\hline $\begin{array}{l}\text { 5-chloro-2-(5-methyl- } \\
\text { 1,4-diazepan-1-yl)-1,3- } \\
\text { benzoxazole }\end{array}$ & $\begin{array}{l}\text { CC1CCN(CCN1)c1nc2cc } \\
\text { (Cl)ccc2o1 }\end{array}$ & & $\begin{array}{l}\text { Non- } \\
\text { sensitizer }\end{array}$ & & & & & REACH (2008-2014) & \\
\hline $\begin{array}{l}\text { 5-cyclohexyl-2- } \\
\text { methylpentan-1-ol }\end{array}$ & $\mathrm{CC}(\mathrm{CO}) \mathrm{CCCC} 1 \mathrm{CCCCC1}$ & & $\begin{array}{l}\text { Non- } \\
\text { sensitizer }\end{array}$ & & & & & REACH (2008-2014) & \\
\hline $\begin{array}{l}\text { 5-ethyl-2,8-dimethyl-5- } \\
\{[(\text { propan-2- } \\
\text { ylidene)amino]oxy\}-4,6- } \\
\text { dioxa-3,7-diaza-5- } \\
\text { silanona-2,7-diene }\end{array}$ & $\begin{array}{l}\mathrm{CC}[\mathrm{Si}](\mathrm{ON}=\mathrm{C}(\mathrm{C}) \mathrm{C})(\mathrm{ON}= \\
\mathrm{C}(\mathrm{C}) \mathrm{C}) \mathrm{ON}=\mathrm{C}(\mathrm{C}) \mathrm{C}\end{array}$ & & $\begin{array}{l}\text { Non- } \\
\text { sensitizer }\end{array}$ & & & & & REACH (2008-2014) & \\
\hline $\begin{array}{l}\text { 5-methyl-2-(2H-1,2,3- } \\
\text { triazol-2-yl)benzoic acid }\end{array}$ & $\begin{array}{l}\text { Cc1ccc(c(c1)C(0)=0)- } \\
\text { n1nccn1 }\end{array}$ & & $\begin{array}{l}\text { Non- } \\
\text { sensitizer }\end{array}$ & & & & & REACH (2008-2014) & \\
\hline $\begin{array}{l}\text { 5-methyl-2-(propan-2- } \\
\text { yl)-N-[2-(pyridin-2- } \\
\text { yl)ethyl]cyclohexane-1- } \\
\text { carboxamide }\end{array}$ & $\begin{array}{l}\mathrm{CC}(\mathrm{C}) \mathrm{C} 1 \mathrm{CCC}(\mathrm{C}) \mathrm{CC} 1 \mathrm{C}= \\
\text { O) NCCc1cccon1 }\end{array}$ & & $\begin{array}{l}\text { Non- } \\
\text { sensitizer }\end{array}$ & & & & & REACH (2008-2014) & \\
\hline $\begin{array}{l}\text { 6,13-dichloro- } \\
\text { 5,7,12,14-tetrahydro- } \\
\text { 5,12-diazapentacene- } \\
\text { 7,14-dione }\end{array}$ & $\begin{array}{l}\mathrm{Clc1} 1 \mathrm{c} 2 \mathrm{Nc} 3 \operatorname{ccccc} 3 \mathrm{C}(=\mathrm{O}) \\
\mathrm{c} 2 \mathrm{c}(\mathrm{Cl}) \mathrm{c} 2 \mathrm{Nc} 3 \operatorname{ccccc} 3 \mathrm{C}(= \\
\mathrm{O}) \mathrm{c} 12\end{array}$ & & $\begin{array}{l}\text { Non- } \\
\text { sensitizer }\end{array}$ & & & & & REACH (2008-2014) & \\
\hline $\begin{array}{l}\text { 6-(4- } \\
\text { methylbenzenesulfona } \\
\text { mido)hexanoic acid }\end{array}$ & $\begin{array}{l}\operatorname{Cc} 1 \operatorname{ccc}(\operatorname{cc} 1) S(=0)(=0) N \\
\operatorname{cccccC}(O)=0\end{array}$ & & $\begin{array}{l}\text { Non- } \\
\text { sensitizer }\end{array}$ & & & & & REACH (2008-2014) & \\
\hline
\end{tabular}




\begin{tabular}{|c|c|c|c|c|c|c|c|c|c|}
\hline $\begin{array}{l}\text { 6-(trifluoro-methyl)- } \\
\text { 2,3-dihydro-5-methyl- } \\
\text { 1h-indole, } \\
\text { hydrochloride }\end{array}$ & $\begin{array}{l}\mathrm{Cc} 1 \mathrm{cc} 2 \mathrm{CCN} 2 \mathrm{cc} 1 \mathrm{C}(\mathrm{F})(\mathrm{F} \\
) \mathrm{F}\end{array}$ & & $\begin{array}{l}\text { Non- } \\
\text { sensitizer }\end{array}$ & & & & & ICCVAM (2013) & \\
\hline 6-Chloro-1-hexanol & OCCCCCCCl & & $\begin{array}{l}\text { Non- } \\
\text { sensitizer }\end{array}$ & & & & & ICCVAM (2013) & \\
\hline 6-Iodo-quinazolin-4-ol & $\begin{array}{l}\text { Ic1ccc2NC=NC(=O)c2c } \\
1\end{array}$ & & $\begin{array}{l}\text { Non- } \\
\text { sensitizer }\end{array}$ & & & & & ICCVAM (2013) & \\
\hline $\begin{array}{l}\text { 6-Methoxy-4-methyl- } \\
\text { 2(1H)-quinolinone }\end{array}$ & $\begin{array}{l}\operatorname{COc} 1 \mathrm{ccc} 2 \mathrm{NC}(=0) \mathrm{C}=\mathrm{C}(\mathrm{C} \\
) \mathrm{c} 2 \mathrm{c} 1\end{array}$ & & $\begin{array}{l}\text { Non- } \\
\text { sensitizer }\end{array}$ & & & & & ICCVAM (2013) & \\
\hline $\begin{array}{l}\text { 6- } \\
\text { Methoxynaphthalene- } \\
\text { 2-carbaldehyde }\end{array}$ & $\begin{array}{l}\operatorname{cOc} 1 \operatorname{ccc} 2 \operatorname{cc}(\mathrm{C}=0) \operatorname{ccc} 2 c \\
1\end{array}$ & & $\begin{array}{l}\text { Non- } \\
\text { sensitizer }\end{array}$ & & & & & ICCVAM (2013) & \\
\hline Compound name & SMILES & $\begin{array}{l}\text { Human } \\
\text { result }\end{array}$ & $\begin{array}{l}\text { LLNA } \\
\text { result }\end{array}$ & DPRA & KeratinoSens & h-CLAT & $\begin{array}{l}\text { Human } \\
\text { reference }\end{array}$ & LLNA reference & Non-animal reference \\
\hline $\begin{array}{l}\text { 6-Phenyl-1,3,5-triazine- } \\
\text { 2,4-diamine }\end{array}$ & $\begin{array}{l}\text { Nc1nc(N)nc(n1)- } \\
\text { c1ccccc1 }\end{array}$ & & $\begin{array}{l}\text { Non- } \\
\text { sensitizer }\end{array}$ & & & & & REACH (2008-2014) & \\
\hline $\begin{array}{l}\text { 6-amino-1,3- } \\
\text { dimethylpyrimidine- } \\
\text { 2,4-dione }\end{array}$ & $\begin{array}{l}C N 1 C(N)=C C(=O) N(C) C \\
1=0\end{array}$ & & $\begin{array}{l}\text { Non- } \\
\text { sensitizer }\end{array}$ & & & & & REACH (2008-2014) & \\
\hline $\begin{array}{l}\text { 7-methyl-3- } \\
\text { methylideneocta-1,6- } \\
\text { diene }\end{array}$ & $\mathrm{CC}(\mathrm{C})=\mathrm{CCCC}(=\mathrm{C}) \mathrm{C}=\mathrm{C}$ & & $\begin{array}{l}\text { Non- } \\
\text { sensitizer }\end{array}$ & & & & & REACH (2008-2014) & \\
\hline $\begin{array}{l}\text { 8,9,10,11-tetrachloro- } \\
\text { 12-phthaloperinone }\end{array}$ & $\begin{array}{l}\mathrm{Clc1} 1 \mathrm{c} 2 \mathrm{C}(=\mathrm{O}) \mathrm{N} 3 \mathrm{C}(=\mathrm{Nc} 4 \\
\operatorname{cccc5} 5 \mathrm{cccc} 3 \mathrm{c} 45) \mathrm{c} 2 \mathrm{c}(\mathrm{Cl}) \mathrm{c} \\
(\mathrm{Cl}) \mathrm{c} 1 \mathrm{Cl}\end{array}$ & & $\begin{array}{l}\text { Non- } \\
\text { sensitizer }\end{array}$ & & & & & REACH (2008-2014) & \\
\hline $\begin{array}{l}\text { 8- } \\
\text { Acetoxycarvotanaceton } \\
\text { e }\end{array}$ & $\begin{array}{l}\mathrm{CC}(=0) O C(C)(C) C 1 C C= \\
C(C) C(=0) C 1\end{array}$ & & $\begin{array}{l}\text { Non- } \\
\text { sensitizer }\end{array}$ & & & & & ICCVAM (2013) & \\
\hline $\begin{array}{l}\text { 8-Amino-6-methoxy-4- } \\
\text { methylquinoline }\end{array}$ & $\begin{array}{l}\operatorname{COc1cc}(\mathrm{N}) \mathrm{c} 2 \mathrm{nccc}(\mathrm{C}) \mathrm{c} 2 \\
\mathrm{c} 1\end{array}$ & & $\begin{array}{l}\text { Non- } \\
\text { sensitizer }\end{array}$ & & & & & ICCVAM (2013) & \\
\hline $\begin{array}{l}\text { 8-Hydroxy-5-[(1R)-1- } \\
\text { hydroxy-2-[[2-[4-[(6- } \\
\text { methoxy[1,1'-biphenyl]- } \\
\text { 3-yl)amino]phenyl]- } \\
\text { ethyl]amino]ethyl]- } \\
\text { 2(1H)-quinolinone }\end{array}$ & $\begin{array}{l}\operatorname{cOc} 1 \operatorname{ccc}(\operatorname{Nc} 2 \operatorname{ccc}(\mathrm{CCNC} \\
\mathrm{C}(\mathrm{O}) \mathrm{c} 3 \operatorname{ccc}(\mathrm{O}) \mathrm{c} 4 \mathrm{NC}(=\mathrm{O}) \\
\mathrm{C}=\mathrm{Cc} 34) \mathrm{cc} 2) \operatorname{cc1} 1- \\
\mathrm{c} 1 \mathrm{ccccc} 1\end{array}$ & & $\begin{array}{l}\text { Non- } \\
\text { sensitizer }\end{array}$ & & & & & ICCVAM (2013) & \\
\hline $\begin{array}{l}\text { 8-[(4-Phthalimido-1- } \\
\text { methylbutyl)amino]- } \\
\text { 2,6-dimethoxy-4- } \\
\text { methyl-5-(3- } \\
\text { trifluoromethylphenoxy } \\
\text { )quinoline }\end{array}$ & $\begin{array}{l}\operatorname{COc1cc(C)c2c(Oc3cccc} \\
\mathrm{c} 3) \mathrm{C}(\mathrm{F})(\mathrm{F}) \mathrm{F}) \mathrm{c}(\mathrm{OC}) \mathrm{cc}(\mathrm{NC} \\
(\mathrm{C}) \mathrm{cCCN} 3 \mathrm{C}(=\mathrm{O}) \mathrm{c} 4 \mathrm{ccccc} \\
4 \mathrm{C} 3=0) \mathrm{c} 2 \mathrm{n} 1\end{array}$ & & $\begin{array}{l}\text { Non- } \\
\text { sensitizer }\end{array}$ & & & & & ICCVAM (2013) & \\
\hline $\begin{array}{l}\text { 8-methylnonyl 2- } \\
\text { methylprop-2-enoate }\end{array}$ & $\begin{array}{l}\mathrm{CC}(\mathrm{C}) \mathrm{CCCCCCCOC}(=0) \\
\mathrm{C}(\mathrm{C})=\mathrm{C}\end{array}$ & & $\begin{array}{l}\text { Non- } \\
\text { sensitizer }\end{array}$ & & & & & REACH (2008-2014) & \\
\hline Adipic acid & $\mathrm{OC}(=0) \operatorname{CCCCC}(0)=0$ & & $\begin{array}{l}\text { Non- } \\
\text { sensitizer }\end{array}$ & & & & & ICCVAM (2013) & \\
\hline
\end{tabular}




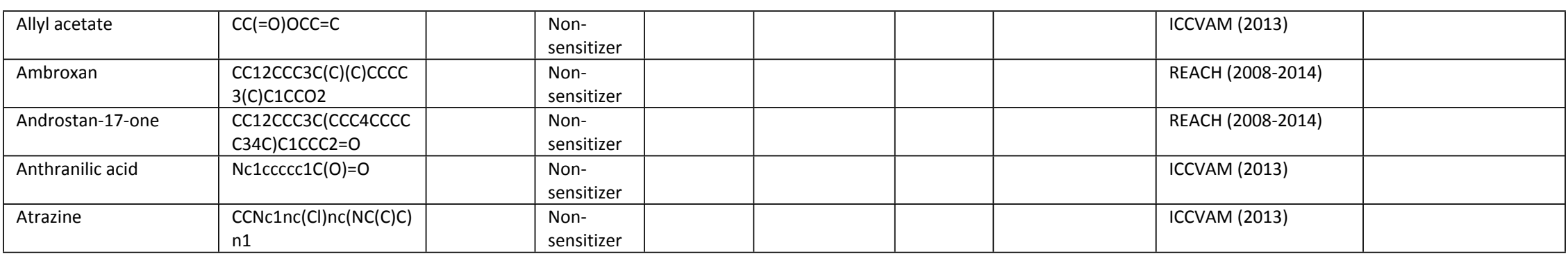

\begin{tabular}{|c|c|c|c|c|c|c|c|c|c|}
\hline Compound name & SMILES & $\begin{array}{l}\text { Human } \\
\text { result }\end{array}$ & $\begin{array}{l}\text { LLNA } \\
\text { result }\end{array}$ & DPRA & KeratinoSens & h-CLAT & $\begin{array}{l}\text { Human } \\
\text { reference }\end{array}$ & LLNA reference & Non-animal reference \\
\hline $\begin{array}{l}\text { Beclomethasone-17- } \\
\text { monopropionate }\end{array}$ & $\begin{array}{l}\mathrm{CCC}(=0) O C 1(\mathrm{C}(\mathrm{C}) \mathrm{CC} 2 \mathrm{C} \\
3 \mathrm{CCC} 4=\mathrm{CC}(=\mathrm{O}) \mathrm{C}=\mathrm{CC} 4(\mathrm{C} \\
) \mathrm{C} 3(\mathrm{Cl}) \mathrm{C}(\mathrm{O}) \mathrm{CC} 12 \mathrm{C}) \mathrm{C}(= \\
\mathrm{O}) \mathrm{CO}\end{array}$ & & $\begin{array}{l}\text { Non- } \\
\text { sensitizer }\end{array}$ & & & & & ICCVAM (2013) & \\
\hline $\begin{array}{l}\text { Benzenepropanol, } \\
\text { acetate }\end{array}$ & $C C(=0) O C C C \operatorname{cocccc} 1$ & & $\begin{array}{l}\text { Non- } \\
\text { sensitizer }\end{array}$ & & & & & ICCVAM (2013) & \\
\hline $\begin{array}{l}\text { Bicyclo[2.2.1]hept-2- } \\
\text { ene }\end{array}$ & $\mathrm{C} 1 \mathrm{CC} 2 \mathrm{CC} 1 \mathrm{C}=\mathrm{C} 2$ & & $\begin{array}{l}\text { Non- } \\
\text { sensitizer }\end{array}$ & & & & & REACH (2008-2014) & \\
\hline $\begin{array}{l}\text { Bis(nonafluorobutyl)ph } \\
\text { osphinic acid }\end{array}$ & $\begin{array}{l}\text { OP(=O)(C(F)(F)C(F)(F)C } \\
(F)(F) C(F)(F) F) C(F)(F) C( \\
F)(F) C(F)(F) C(F)(F) F\end{array}$ & & $\begin{array}{l}\text { Non- } \\
\text { sensitizer }\end{array}$ & & & & & REACH (2008-2014) & \\
\hline Carbon disulfide & $\mathrm{S}=\mathrm{C}=\mathrm{S}$ & & $\begin{array}{l}\text { Non- } \\
\text { sensitizer }\end{array}$ & & & & & REACH (2008-2014) & \\
\hline Chlorpyrifos & $\begin{array}{l}\text { CCOP(=S)(OCC)Oc1ncl } \\
\mathrm{Cl}) \mathrm{c}(\mathrm{Cl}) \mathrm{cc} 1 \mathrm{Cl}\end{array}$ & & $\begin{array}{l}\text { Non- } \\
\text { sensitizer }\end{array}$ & & & & & ICCVAM (2013) & \\
\hline Cholesterol & $\begin{array}{l}\mathrm{CC}(\mathrm{C}) \mathrm{CCCC}(\mathrm{C}) \mathrm{C} 1 \mathrm{CCC} 2 \mathrm{C} \\
3 \mathrm{CC}=\mathrm{C} 4 \mathrm{CC}(\mathrm{O}) \mathrm{CCC} 4(\mathrm{C}) \mathrm{C} \\
3 \mathrm{CCC} 12 \mathrm{C}\end{array}$ & & $\begin{array}{l}\text { Non- } \\
\text { sensitizer }\end{array}$ & & & & & REACH (2008-2014) & \\
\hline $\begin{array}{l}\text { Cinnamyl } \\
\text { hexopyranoside }\end{array}$ & $\begin{array}{l}\mathrm{OCC} 10 \mathrm{OC}(\mathrm{OCC}=\mathrm{Cc} 2 \mathrm{cccc} \\
\mathrm{c} 2) \mathrm{C}(\mathrm{O}) \mathrm{C}(\mathrm{O}) \mathrm{C} 10\end{array}$ & & $\begin{array}{l}\text { Non- } \\
\text { sensitizer }\end{array}$ & & & & & REACH (2008-2014) & \\
\hline Clarithromycin & $\begin{array}{l}\text { CCC1OC(=O)C(C)C(OC2 } \\
\mathrm{CC}(\mathrm{C})(\mathrm{OC}) \mathrm{C}(\mathrm{O}) \mathrm{C}(\mathrm{C}) \mathrm{O} 2) \\
\mathrm{C}(\mathrm{C}) \mathrm{C}(\mathrm{OC} 2 \mathrm{OC}(\mathrm{C}) \mathrm{CC}(\mathrm{C} 2 \\
\mathrm{O}) \mathrm{N}(\mathrm{C}) \mathrm{C}) \mathrm{C}(\mathrm{C})(\mathrm{CC}(\mathrm{C}) \mathrm{C}(= \\
\mathrm{O}) \mathrm{C}(\mathrm{C}) \mathrm{C}(\mathrm{O}) \mathrm{C1}(\mathrm{C}) \mathrm{O}) \mathrm{OC}\end{array}$ & & $\begin{array}{l}\text { Non- } \\
\text { sensitizer }\end{array}$ & & & & & ICCVAM (2013) & \\
\hline Crotonyl thioglycerol & $\mathrm{OCC}(\mathrm{O}) \mathrm{CSC}(=\mathrm{O}) \mathrm{CC}=\mathrm{C}$ & & $\begin{array}{l}\text { Non- } \\
\text { sensitizer }\end{array}$ & & & & & ICCVAM (2013) & \\
\hline Cyclohexane & C1CCCCC1 & & $\begin{array}{l}\text { Non- } \\
\text { sensitizer }\end{array}$ & & & & & REACH (2008-2014) & \\
\hline Cyclooctanol & oc1CCCCCCC1 & & $\begin{array}{l}\text { Non- } \\
\text { sensitizer }\end{array}$ & & & & & ICCVAM (2013) & \\
\hline
\end{tabular}




\begin{tabular}{|c|c|c|c|c|c|c|c|c|c|}
\hline Cyclopentanepropanol & OCCCC1CCCC1 & & $\begin{array}{l}\text { Non- } \\
\text { sensitizer }\end{array}$ & & & & & ICCVAM (2013) & \\
\hline Cytosine hemihydrate & $\mathrm{NC} 1=\mathrm{NC}(=\mathrm{O}) \mathrm{NC}=\mathrm{C} 1$ & & $\begin{array}{l}\text { Non- } \\
\text { sensitizer }\end{array}$ & & & & & ICCVAM (2013) & \\
\hline D-Isosorbide & $\mathrm{OC} 1 \mathrm{COC} 2 \mathrm{C}(\mathrm{O}) \mathrm{COC} 12$ & & $\begin{array}{l}\text { Non- } \\
\text { sensitizer }\end{array}$ & & & & & REACH (2008-2014) & \\
\hline $\begin{array}{l}\text { Diallyldimethylammoni } \\
\text { um chloride }\end{array}$ & $\mathrm{C}[\mathrm{N}+](\mathrm{C})(\mathrm{CC}=\mathrm{C}) \mathrm{CC}=\mathrm{C}$ & & $\begin{array}{l}\text { Non- } \\
\text { sensitizer }\end{array}$ & & & & & REACH (2008-2014) & \\
\hline $\begin{array}{l}\text { Diaminophosphoryloxy } \\
\text { benzene }\end{array}$ & $N P(N)(=0) O c 1 \operatorname{ccccc} 1$ & & $\begin{array}{l}\text { Non- } \\
\text { sensitizer }\end{array}$ & & & & & REACH (2008-2014) & \\
\hline $\begin{array}{l}\text { Diazanium 2-(2-oxido-2- } \\
\text { oxoethyl)disulfanylacet } \\
\text { ate }\end{array}$ & $\mathrm{OC}(=0) \operatorname{csscC}(0)=0$ & & $\begin{array}{l}\text { Non- } \\
\text { sensitizer }\end{array}$ & & & & & REACH (2008-2014) & \\
\hline Compound name & SMILES & $\begin{array}{l}\text { Human } \\
\text { result }\end{array}$ & $\begin{array}{l}\text { LLNA } \\
\text { result }\end{array}$ & DPRA & KeratinoSens & h-CLAT & $\begin{array}{l}\text { Human } \\
\text { reference }\end{array}$ & LLNA reference & Non-animal reference \\
\hline Dibromoanthanthrone & $\begin{array}{l}\mathrm{Brc} 1 \mathrm{cc} 2 \mathrm{C}(=0) \operatorname{c3cccc} 4 \mathrm{c}( \\
\mathrm{Br}) \operatorname{cc} 5 \mathrm{C}(=0) \operatorname{coc} c \operatorname{cc} 1 \mathrm{c} 6 \mathrm{c} \\
2-\mathrm{c} 5 \mathrm{c} 34\end{array}$ & & $\begin{array}{l}\text { Non- } \\
\text { sensitizer }\end{array}$ & & & & & REACH (2008-2014) & \\
\hline Dichloromethane & $\mathrm{ClCCl}$ & & $\begin{array}{l}\text { Non- } \\
\text { sensitizer }\end{array}$ & & & & & REACH (2008-2014) & \\
\hline Diethyl carbonate & $\mathrm{CCOC}(=0) \mathrm{OCC}$ & & $\begin{array}{l}\text { Non- } \\
\text { sensitizer }\end{array}$ & & & & & REACH (2008-2014) & \\
\hline $\begin{array}{l}\text { Dimethyl 4-cyano-4-(3- } \\
\text { cyclopentyloxy-4- } \\
\text { methoxyphenyl)- } \\
\text { pimelate }\end{array}$ & $\begin{array}{l}\mathrm{COC}(=\mathrm{O}) \operatorname{ccC}(\mathrm{CCC}(=\mathrm{O}) \\
\mathrm{OC})(\mathrm{C \# N}) \mathrm{c} 1 \mathrm{ccc}(\mathrm{OC}) \mathrm{c}(\mathrm{O} \\
\mathrm{C} 2 \mathrm{CCCC} 2) \mathrm{c} 1\end{array}$ & & $\begin{array}{l}\text { Non- } \\
\text { sensitizer }\end{array}$ & & & & & ICCVAM (2013) & \\
\hline Dimethyl butanedioate & $\operatorname{COC}(=0) \mathrm{CCC}(=0) O C$ & & $\begin{array}{l}\text { Non- } \\
\text { sensitizer }\end{array}$ & & & & & REACH (2008-2014) & \\
\hline Dimethyl carbonate & $\mathrm{COC}(=0) O C$ & & $\begin{array}{l}\text { Non- } \\
\text { sensitizer }\end{array}$ & & & & & ICCVAM (2013) & \\
\hline Dimethyl isophthalate & $\begin{array}{l}\operatorname{cOC}(=0) \operatorname{c1} \operatorname{cccc}(\mathrm{c} 1) \mathrm{C}(= \\
\text { O)OC }\end{array}$ & & $\begin{array}{l}\text { Non- } \\
\text { sensitizer }\end{array}$ & & & & & $\begin{array}{l}\text { ICCVAM }(2013)+ \\
\text { REACH }(2008-2014)\end{array}$ & \\
\hline $\begin{array}{l}\text { Dimethyl } \\
\text { pentanedioate }\end{array}$ & $\operatorname{cOC}(=0) \operatorname{CCCC}(=0) O C$ & & $\begin{array}{l}\text { Non- } \\
\text { sensitizer }\end{array}$ & & & & & REACH (2008-2014) & \\
\hline Dimethyl sulfone & $\operatorname{cs}(C)(=0)=0$ & & $\begin{array}{l}\text { Non- } \\
\text { sensitizer }\end{array}$ & & & & & ICCVAM (2013) & \\
\hline $\begin{array}{l}\text { Disodium sulfite } \\
\text { heptahydrate }\end{array}$ & $O S(O)=0$ & & $\begin{array}{l}\text { Non- } \\
\text { sensitizer }\end{array}$ & & & & & REACH (2008-2014) & \\
\hline Equol & $\begin{array}{l}\text { Oc1ccc(cc1)C1COc2ccl } \\
\text { O)ccc2 } 2 \text { 1 } 1\end{array}$ & & $\begin{array}{l}\text { Non- } \\
\text { sensitizer }\end{array}$ & & & & & ICCVAM (2013) & \\
\hline Ethenyl acetate & $\mathrm{CC}(=0) \mathrm{OC}=\mathrm{C}$ & & $\begin{array}{l}\text { Non- } \\
\text { sensitizer }\end{array}$ & & & & & REACH (2008-2014) & \\
\hline Ethenyl dodecanoate & $\begin{array}{l}\text { CCCCCCCCCCCC }(=0) O C \\
=C\end{array}$ & & $\begin{array}{l}\text { Non- } \\
\text { sensitizer }\end{array}$ & & & & & REACH (2008-2014) & \\
\hline Ethoxyethane & CCOCC & & $\begin{array}{l}\text { Non- } \\
\text { sensitizer }\end{array}$ & & & & & REACH (2008-2014) & \\
\hline
\end{tabular}




\begin{tabular}{|c|c|c|c|c|c|c|c|c|c|}
\hline $\begin{array}{l}\text { Ethyl (Z)- } \alpha \text {-((2-(tert- } \\
\text { butoxy)-1,1-dimethyl-2- } \\
\text { oxoethoxy)imino)-2- } \\
\text { (tritylamino)thiazol-4- } \\
\text { acetate }\end{array}$ & $\begin{array}{l}\mathrm{CCOC}(=0) \mathrm{C}(=\mathrm{NOC}(\mathrm{C})(\mathrm{C} \\
\mathrm{C}(=\mathrm{O}) \mathrm{OC}(\mathrm{C})(\mathrm{C}) \mathrm{C}) \mathrm{c} 1 \mathrm{csc}( \\
\mathrm{NC}(\mathrm{c} 2 \mathrm{ccccc} 2)(\mathrm{c} 2 \operatorname{ccccc} 2 \\
) \operatorname{c} 2 \operatorname{ccccc} 2) \mathrm{n} 1\end{array}$ & & $\begin{array}{l}\text { Non- } \\
\text { sensitizer }\end{array}$ & & & & & ICCVAM (2013) & \\
\hline $\begin{array}{l}\text { Ethyl } 1 \mathrm{H} \text {-1,2,4-triazole- } \\
\text { 3-carboxylate }\end{array}$ & $\mathrm{CCOC}(=0) \mathrm{c} 1 \mathrm{nnc}[\mathrm{nH}] 1$ & & $\begin{array}{l}\text { Non- } \\
\text { sensitizer }\end{array}$ & & & & & ICCVAM (2013) & \\
\hline $\begin{array}{l}\text { Ethyl 2,6-dichloro-5- } \\
\text { fluoro- } \beta \text {-oxo-3- } \\
\text { pyridinepropanoate }\end{array}$ & $\begin{array}{l}\mathrm{CCOC}(=0) \mathrm{CC}(=0) \mathrm{c} 1 \mathrm{cc}(\mathrm{F} \\
) \mathrm{c}(\mathrm{Cl}) \mathrm{nc} 1 \mathrm{Cl}\end{array}$ & & $\begin{array}{l}\text { Non- } \\
\text { sensitizer }\end{array}$ & & & & & ICCVAM (2013) & \\
\hline Formulation 21 (Sonet) & $\begin{array}{l}\mathrm{FC}(\mathrm{F}) \mathrm{C}(\mathrm{F})(\mathrm{F}) \mathrm{Oc} 1 \mathrm{c}(\mathrm{Cl}) \mathrm{cc}( \\
\mathrm{NC}(=\mathrm{O}) \mathrm{NC}(=\mathrm{O}) \mathrm{c} 2 \mathrm{c}(\mathrm{F}) \mathrm{cc} \\
\mathrm{cc} 2 \mathrm{~F}) \mathrm{cc} 1 \mathrm{Cl}\end{array}$ & & $\begin{array}{l}\text { Non- } \\
\text { sensitizer }\end{array}$ & & & & & ICCVAM (2013) & \\
\hline Compound name & SMILES & $\begin{array}{l}\text { Human } \\
\text { result }\end{array}$ & $\begin{array}{l}\text { LLNA } \\
\text { result }\end{array}$ & DPRA & KeratinoSens & h-CLAT & $\begin{array}{l}\text { Human } \\
\text { reference }\end{array}$ & LLNA reference & Non-animal reference \\
\hline $\begin{array}{l}\text { Formulation } 34 \\
\text { (Aminopyralid) } \\
\end{array}$ & $\begin{array}{l}\mathrm{Nc1} 1 \mathrm{cc}(\mathrm{Cl}) \mathrm{nc}(\mathrm{C}(\mathrm{O})=0) \mathrm{c1} \\
\mathrm{Cl}\end{array}$ & & $\begin{array}{l}\text { Non- } \\
\text { sensitizer }\end{array}$ & & & & & ICCVAM (2013) & \\
\hline $\begin{array}{l}\text { Formulation } 4 \\
\text { (Clopyralid) }\end{array}$ & $\mathrm{OC}(=0) \mathrm{c} 1 \mathrm{nc}(\mathrm{Cl}) \mathrm{ccc} 1 \mathrm{Cl}$ & & $\begin{array}{l}\text { Non- } \\
\text { sensitizer }\end{array}$ & & & & & ICCVAM (2013) & \\
\hline $\begin{array}{l}\text { Furan-2,5-dicarboxylic } \\
\text { acid }\end{array}$ & $\begin{array}{l}\mathrm{OC}(=0) \operatorname{c1ccc}(01) \mathrm{C}(0)= \\
\mathrm{O}\end{array}$ & & $\begin{array}{l}\text { Non- } \\
\text { sensitizer }\end{array}$ & & & & & REACH (2008-2014) & \\
\hline Geranyl nitrile & $\mathrm{CC}(\mathrm{C})=\mathrm{CCCC}(\mathrm{C})=\mathrm{CC} \# \mathrm{~N}$ & & $\begin{array}{l}\text { Non- } \\
\text { sensitizer }\end{array}$ & & & & & ICCVAM (2013) & \\
\hline Heptan-2-one & $\operatorname{ccccc}(C)=0$ & & $\begin{array}{l}\text { Non- } \\
\text { sensitizer }\end{array}$ & & & & & REACH (2008-2014) & \\
\hline $\begin{array}{l}\text { Hexadecoxycarbonylox } \\
\text { y hexadecyl carbonate }\end{array}$ & $\begin{array}{l}\text { CCCCCCCCCCCCCCCCO } \\
\text { C(=O)OOC(=O)OCCCCC } \\
\text { CCCCCCCCCCC }\end{array}$ & & $\begin{array}{l}\text { Non- } \\
\text { sensitizer }\end{array}$ & & & & & REACH (2008-2014) & \\
\hline Hexanedihydrazide & $\mathrm{NNC}(=0) \mathrm{CCCCC}(=0) \mathrm{NN}$ & & $\begin{array}{l}\text { Non- } \\
\text { sensitizer }\end{array}$ & & & & & REACH (2008-2014) & \\
\hline Indanthrene blue & $\begin{array}{l}\mathrm{O}=\mathrm{C} 1 \mathrm{c} 2 \operatorname{ccccc} 2 \mathrm{C}(=0) \mathrm{c} 2 \\
\mathrm{c} 3 \mathrm{~N} c 4 \operatorname{ccc} 5 \mathrm{C}(=0) \operatorname{coc} 6 \mathrm{ccc} \\
\mathrm{c} 6 \mathrm{C}(=0) \cos 4 \mathrm{c}) 3 \mathrm{ccc} 12\end{array}$ & & $\begin{array}{l}\text { Non- } \\
\text { sensitizer }\end{array}$ & & & & & REACH (2008-2014) & \\
\hline Isoindole-1,3-dione & $\mathrm{O}=\mathrm{C} 1 \mathrm{NC}(=0) \operatorname{coc} \operatorname{cccc} 12$ & & $\begin{array}{l}\text { Non- } \\
\text { sensitizer }\end{array}$ & & & & & REACH (2008-2014) & \\
\hline $\begin{array}{l}\text { Isopropyl } \\
\text { dicyandiamide }\end{array}$ & $\mathrm{CC}(\mathrm{C}) \mathrm{N}(\mathrm{C \# N}) \mathrm{CHN}$ & & $\begin{array}{l}\text { Non- } \\
\text { sensitizer }\end{array}$ & & & & & ICCVAM (2013) & \\
\hline Isopropyl eugenol & $\begin{array}{l}\mathrm{CC}(\mathrm{C}) \operatorname{Oc} 1 \mathrm{cc}(\mathrm{CC}=\mathrm{C}) \mathrm{ccc} 1 \\
\mathrm{O}\end{array}$ & & $\begin{array}{l}\text { Non- } \\
\text { sensitizer }\end{array}$ & & & & & ICCVAM (2013) & \\
\hline Isopropyl glycerol ether & $\mathrm{CC}(\mathrm{C}) \mathrm{OCC}(\mathrm{O}) \mathrm{CO}$ & & $\begin{array}{l}\text { Non- } \\
\text { sensitizer }\end{array}$ & & & & & ICCVAM (2013) & \\
\hline Linalool alcohol & $\begin{array}{l}\mathrm{CC}(\mathrm{C})(\mathrm{O}) \mathrm{C}=\mathrm{CCC}(\mathrm{C})(\mathrm{O}) \mathrm{C} \\
=\mathrm{C}\end{array}$ & & $\begin{array}{l}\text { Non- } \\
\text { sensitizer }\end{array}$ & & & & & ICCVAM (2013) & \\
\hline Lipase, triacylglycerol & $\begin{array}{l}\mathrm{OC} 1=\mathrm{CC}(=\mathrm{O}) \mathrm{C}=\mathrm{CC} 1=\mathrm{N} \\
\mathrm{Nc} 1 \mathrm{cccc} 1\end{array}$ & & $\begin{array}{l}\text { Non- } \\
\text { sensitizer }\end{array}$ & & & & & REACH (2008-2014) & \\
\hline
\end{tabular}




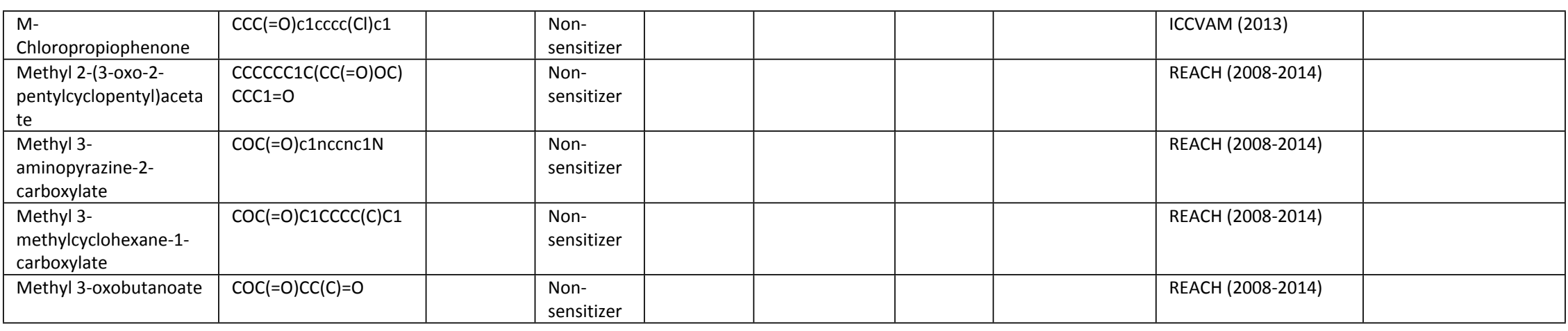

\begin{tabular}{|c|c|c|c|c|c|c|c|c|c|}
\hline Compound name & SMILES & $\begin{array}{l}\text { Human } \\
\text { result }\end{array}$ & $\begin{array}{l}\text { LLNA } \\
\text { result }\end{array}$ & DPRA & KeratinoSens & h-CLAT & $\begin{array}{l}\text { Human } \\
\text { reference }\end{array}$ & LLNA reference & Non-animal reference \\
\hline $\begin{array}{l}\text { Methyl 5-amino-4- } \\
\text { cyano-3- } \\
\text { methylthiophene-2- } \\
\text { carboxylate }\end{array}$ & $\begin{array}{l}\mathrm{COC}(=\mathrm{O}) \mathrm{c} 1 \mathrm{sc}(\mathrm{N}) \mathrm{c}(\mathrm{C \# N}) \\
\mathrm{c} 1 \mathrm{C}\end{array}$ & & $\begin{array}{l}\text { Non- } \\
\text { sensitizer }\end{array}$ & & & & & REACH (2008-2014) & \\
\hline Methyl benzoate & $\operatorname{coC}(=0) \operatorname{c1ccccc1}$ & & $\begin{array}{l}\text { Non- } \\
\text { sensitizer }\end{array}$ & & & & & REACH (2008-2014) & \\
\hline $\begin{array}{l}\text { Methyl hexadecyl } \\
\text { sulfonate }\end{array}$ & $\begin{array}{l}\text { ccCCCCCCCCCCCCCCS( } \\
=0)(=0) O C\end{array}$ & & $\begin{array}{l}\text { Non- } \\
\text { sensitizer }\end{array}$ & & & & & ICCVAM (2013) & \\
\hline $\begin{array}{l}\text { Morpholin-4-yl } \\
\text { morpholine-4- } \\
\text { carbodithioate }\end{array}$ & $\begin{array}{l}\mathrm{S}=\mathrm{C}(\mathrm{SN} 1 \mathrm{CCOCC} 1) \mathrm{N} 1 \mathrm{CC} \\
\mathrm{OCC} 1\end{array}$ & & $\begin{array}{l}\text { Non- } \\
\text { sensitizer }\end{array}$ & & & & & REACH (2008-2014) & \\
\hline $\begin{array}{l}\mathrm{N}, \mathrm{N} \text {-Dimethyl-3- } \\
\text { oxobutanamide }\end{array}$ & $\mathrm{CN}(\mathrm{C}) \mathrm{C}(=\mathrm{O}) \mathrm{CC}(\mathrm{C})=\mathrm{O}$ & & $\begin{array}{l}\text { Non- } \\
\text { sensitizer }\end{array}$ & & & & & REACH (2008-2014) & \\
\hline $\begin{array}{l}\mathrm{N}, \mathrm{N} \text {-dimethyloctan-1- } \\
\text { amine oxide }\end{array}$ & $\begin{array}{l}\operatorname{CCCCCCCC}[N+](C)(C)[0 \\
-]\end{array}$ & & $\begin{array}{l}\text { Non- } \\
\text { sensitizer }\end{array}$ & & & & & REACH (2008-2014) & \\
\hline $\begin{array}{l}\text { N,N-dimethylpropan-1- } \\
\text { amine }\end{array}$ & $\mathrm{CCCN}(\mathrm{C}) \mathrm{C}$ & & $\begin{array}{l}\text { Non- } \\
\text { sensitizer }\end{array}$ & & & & & REACH (2008-2014) & \\
\hline $\begin{array}{l}\mathrm{N}-(2,2- \\
\text { dimethoxyethyl)prop-2- } \\
\text { enamide }\end{array}$ & $\operatorname{COC}(\mathrm{CNC}(=\mathrm{O}) \mathrm{C}=\mathrm{C}) \mathrm{OC}$ & & $\begin{array}{l}\text { Non- } \\
\text { sensitizer }\end{array}$ & & & & & REACH (2008-2014) & \\
\hline $\begin{array}{l}\mathrm{N} \text {-(2,6-difluorophenyl)- } \\
5 \text {-methyl- } \\
{[1,2,4] \text { triazolo[1,5- }} \\
\text { a]pyrimidine-2- } \\
\text { sulfonamide }\end{array}$ & $\begin{array}{l}\mathrm{Cc} 1 \mathrm{ccn} 2 \mathrm{nc}(\mathrm{nc} 2 \mathrm{n} 1) \mathrm{S}(=\mathrm{O} \\
)(=0) \mathrm{Nc} 1 \mathrm{c}(\mathrm{F}) \operatorname{cccc} 1 \mathrm{~F}\end{array}$ & & $\begin{array}{l}\text { Non- } \\
\text { sensitizer }\end{array}$ & & & & & ICCVAM (2013) & \\
\hline $\begin{array}{l}\mathrm{N} \text {-(2-chloro-4- } \\
\text { pyrimidinyl)-2,3- } \\
\text { dimethyl-2H-indazol-6- } \\
\text { amine }\end{array}$ & $\begin{array}{l}\text { Cc1n(C)nc2cc(Nc3ccnc } \\
(\mathrm{Cl}) \mathrm{n} 3) \mathrm{ccc} 12\end{array}$ & & $\begin{array}{l}\text { Non- } \\
\text { sensitizer }\end{array}$ & & & & & ICCVAM (2013) & \\
\hline
\end{tabular}




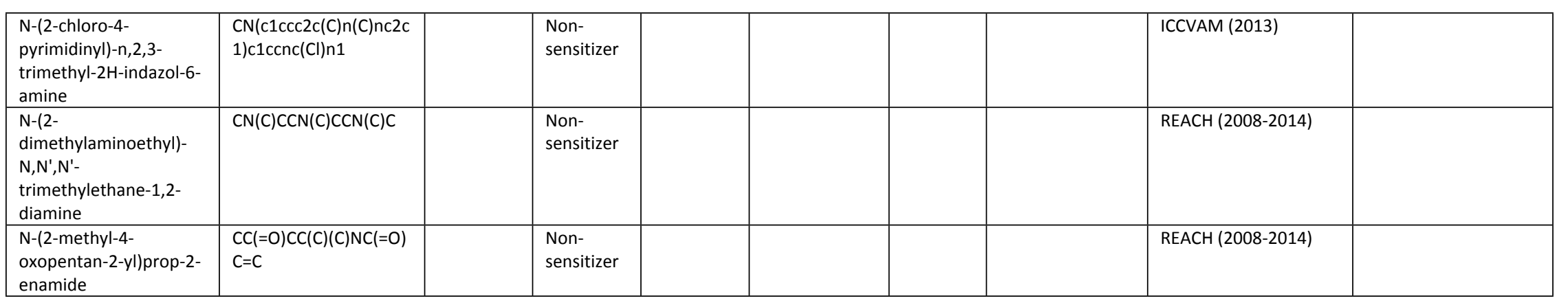

\begin{tabular}{|c|c|c|c|c|c|c|c|c|c|}
\hline Compound name & SMILES & $\begin{array}{l}\text { Human } \\
\text { result }\end{array}$ & $\begin{array}{l}\text { LLNA } \\
\text { result }\end{array}$ & DPRA & KeratinoSens & h-CLAT & $\begin{array}{l}\text { Human } \\
\text { reference }\end{array}$ & LLNA reference & Non-animal reference \\
\hline $\begin{array}{l}\text { N-(4-carbamoylphenyl)- } \\
\text { 4-[1,3-dioxo-1-[(2-oxo- } \\
\text { 1,3- } \\
\text { dihydrobenzimidazol-5- } \\
\text { yl)amino]butan-2- } \\
\text { yl]diazenylbenzamide }\end{array}$ & $\begin{array}{l}\mathrm{CC}(=0) \mathrm{C}(=\mathrm{NNC} 1 \mathrm{ccc}(\mathrm{cc} 1 \\
\mathrm{C}(=\mathrm{O}) \mathrm{Nc1} \operatorname{ccc}(\mathrm{cc} 1) \mathrm{C}(\mathrm{N}) \\
=\mathrm{O}) \mathrm{C}(=0) \mathrm{Nc} 1 \mathrm{ccc} 2 \mathrm{NC}(= \\
\mathrm{O}) \mathrm{Nc} 2 \mathrm{c} 1\end{array}$ & & $\begin{array}{l}\text { Non- } \\
\text { sensitizer }\end{array}$ & & & & & REACH (2008-2014) & \\
\hline $\begin{array}{l}\mathrm{N}-(4-\text { chloro-2,5- } \\
\text { dimethoxyphenyl)-2- } \\
{[2,5 \text {-dimethoxy-4- }} \\
\text { (phenylsulfamoyl)phen } \\
\text { yl]diazenyl-3- } \\
\text { oxobutanamide }\end{array}$ & $\begin{array}{l}\operatorname{cOc} 1 \mathrm{cc}(\mathrm{NC}(=\mathrm{O}) \mathrm{C}(=\mathrm{NNc} \\
2 \mathrm{cc}(\mathrm{OC}) \mathrm{c}(\mathrm{cc} 2 \mathrm{OC}) \mathrm{S}(=\mathrm{O})( \\
=0) \mathrm{Nc} 2 \mathrm{ccccc} 2) \mathrm{C}(\mathrm{C})=0) \\
\mathrm{c}(\mathrm{OC}) \mathrm{cc} 1 \mathrm{Cl}\end{array}$ & & $\begin{array}{l}\text { Non- } \\
\text { sensitizer }\end{array}$ & & & & & REACH (2008-2014) & \\
\hline $\begin{array}{l}\text { N-(4-chloro-2,5- } \\
\text { dimethoxyphenyl)-2-\{2- } \\
{[3,3 \text { '-dichloro-4'-(2-\{1- }} \\
{[(2,4-} \\
\text { dimethylphenyl)carbam } \\
\text { oyl]-2- } \\
\text { oxopropylidene }\} \text { hydrazi } \\
\text { n-1-yl)-[1,1'-biphenyl]- } \\
\text { 4-yl]hydrazin-1- } \\
\text { ylidene\}-3- } \\
\text { oxobutanamide }\end{array}$ & $\begin{array}{l}\operatorname{COc1} 1 \mathrm{cc}(\mathrm{NC}(=\mathrm{O}) \mathrm{C}(=\mathrm{NNc} \\
2 \operatorname{ccc}(\mathrm{cc} 2 \mathrm{Cl})- \\
\operatorname{c2ccc}(\mathrm{NN}=\mathrm{C}(\mathrm{C}(\mathrm{C})=\mathrm{O}) \mathrm{Cl} \\
=0) \mathrm{Nc} 3 \operatorname{ccc}(\mathrm{C}) \mathrm{cc} 3 \mathrm{C}) \mathrm{c}(\mathrm{Cl} \\
\operatorname{c2} 2) \mathrm{C}(\mathrm{C})=\mathrm{O}) \mathrm{c}(\mathrm{OC}) \mathrm{cc1Cl}\end{array}$ & & $\begin{array}{l}\text { Non- } \\
\text { sensitizer }\end{array}$ & & & & & REACH (2008-2014) & \\
\hline $\begin{array}{l}\mathrm{N} \text {-(4-chloro-2,5- } \\
\text { dimethoxyphenyl)-3- } \\
\text { oxobutanamide }\end{array}$ & $\begin{array}{l}\operatorname{COc} 1 \mathrm{cc}(\mathrm{NC}(=\mathrm{O}) \mathrm{CC}(\mathrm{C})= \\
\mathrm{O}) \mathrm{c}(\mathrm{OC}) \operatorname{cc} 1 \mathrm{Cl}\end{array}$ & & $\begin{array}{l}\text { Non- } \\
\text { sensitizer }\end{array}$ & & & & & REACH (2008-2014) & \\
\hline $\begin{array}{l}\mathrm{N} \text {-(5-chloro-2- } \\
\text { methoxyphenyl)-2-(2- } \\
\text { methoxy-4- } \\
\text { nitrophenyl)diazenyl-3- } \\
\text { oxobutanamide }\end{array}$ & $\begin{array}{l}\operatorname{cOc} 1 \operatorname{ccc}(C l) \operatorname{cc} 1 N C(=0) \\
C(=N N \operatorname{coc}(\operatorname{cc} 1 O C) N( \\
=0)=O) C(C)=0\end{array}$ & & $\begin{array}{l}\text { Non- } \\
\text { sensitizer }\end{array}$ & & & & & REACH (2008-2014) & \\
\hline
\end{tabular}




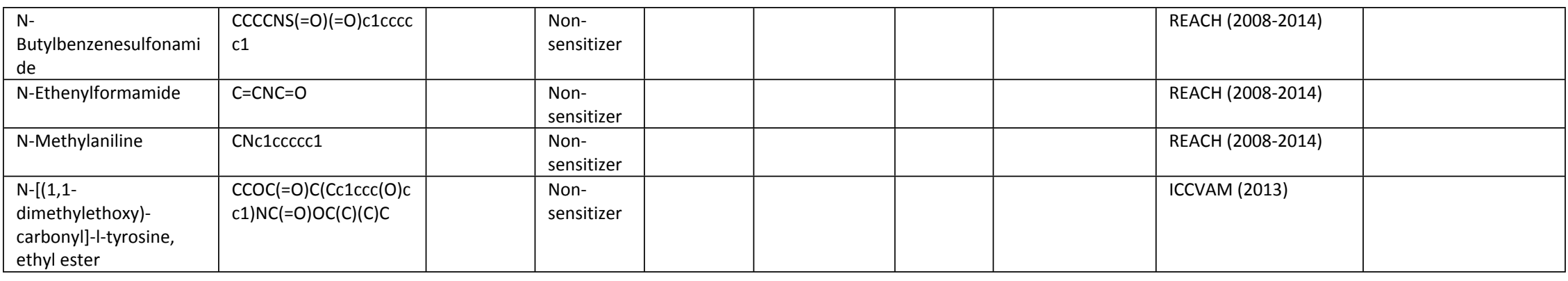

\begin{tabular}{|c|c|c|c|c|c|c|c|c|c|}
\hline Compound name & SMILES & $\begin{array}{l}\text { Human } \\
\text { result }\end{array}$ & $\begin{array}{l}\text { LLNA } \\
\text { result }\end{array}$ & DPRA & KeratinoSens & h-CLAT & $\begin{array}{l}\text { Human } \\
\text { reference }\end{array}$ & LLNA reference & Non-animal reference \\
\hline $\begin{array}{l}\mathrm{N}-[(1-\text { butyl-4- } \\
\text { piperidinyl)methyl]-3,4- } \\
\text { dihydro-2h- } \\
{[1,3] \text { oxazino[3,2- }} \\
\text { a]indole-10- } \\
\text { carboxamide }\end{array}$ & $\begin{array}{l}\text { CCCCN1CCC }(\mathrm{CNC}(=0) \mathrm{C} \\
2 \mathrm{c} 30 \mathrm{CcCn} 3 \mathrm{c} 3 \operatorname{ccccc} 23) \\
\mathrm{CC} 1\end{array}$ & & $\begin{array}{l}\text { Non- } \\
\text { sensitizer }\end{array}$ & & & & & ICCVAM (2013) & \\
\hline $\begin{array}{l}\mathrm{N}-[11- \\
\text { (dichloromethylidene)tr } \\
\text { icyclo[6.2.1.02,7]undeca } \\
\text {-2(7),3,5-trien-3-yl]-3- } \\
\text { (difluoromethyl)-1- } \\
\text { methyl-1H-pyrazole-4- } \\
\text { carboxamide }\end{array}$ & $\begin{array}{l}\mathrm{Cn} 1 \mathrm{cc}(\mathrm{C}(=\mathrm{O}) \mathrm{Nc} 2 \operatorname{cccc} 3 \mathrm{C} \\
4 \mathrm{CCC}(\mathrm{C} 4=\mathrm{C}(\mathrm{Cl}) \mathrm{Cl}) \mathrm{c} 23) \mathrm{c}( \\
\mathrm{n} 1) \mathrm{C}(\mathrm{F}) \mathrm{F}\end{array}$ & & $\begin{array}{l}\text { Non- } \\
\text { sensitizer }\end{array}$ & & & & & REACH (2008-2014) & \\
\hline $\begin{array}{l}\mathrm{N} \text {-[2-benzyloxy-5-(2- } \\
\text { bromo-1-hydroxy- } \\
\text { ethyl)-phenyl]- } \\
\text { formamide }\end{array}$ & $\begin{array}{l}\mathrm{OC}(\mathrm{CBr}) \mathrm{c} 1 \mathrm{ccc}(\mathrm{OCc} 2 \mathrm{ccc} \\
\mathrm{cc} 2) \mathrm{c}(\mathrm{NC}=0) \mathrm{c} 1\end{array}$ & & $\begin{array}{l}\text { Non- } \\
\text { sensitizer }\end{array}$ & & & & & ICCVAM (2013) & \\
\hline $\begin{array}{l}\mathrm{N}- \\
\text { cyclohexylidenehydroxy } \\
\text { lamine }\end{array}$ & $\mathrm{ON}=\mathrm{C} 1 \mathrm{CCCCC} 1$ & & $\begin{array}{l}\text { Non- } \\
\text { sensitizer }\end{array}$ & & & & & REACH (2008-2014) & \\
\hline $\begin{array}{l}\mathrm{N}- \\
\text { octadecyloctadecanami } \\
\text { de }\end{array}$ & $\begin{array}{l}\text { CCCCCCCCCCCCCCCCC } \\
\text { CNC(=O) }) C C C C C C C C C C \\
\text { CCCCCC }\end{array}$ & & $\begin{array}{l}\text { Non- } \\
\text { sensitizer }\end{array}$ & & & & & REACH (2008-2014) & \\
\hline $\begin{array}{l}\text { N-propan-2- } \\
\text { ylhydroxylamine; } \\
\text { sulfuric acid }\end{array}$ & $\mathrm{CC}(\mathrm{C}) \mathrm{NO}$ & & $\begin{array}{l}\text { Non- } \\
\text { sensitizer }\end{array}$ & & & & & REACH (2008-2014) & \\
\hline $\begin{array}{l}\text { N-propan-2- } \\
\text { ylidenehydroxylamine }\end{array}$ & $\mathrm{CC}(\mathrm{C})=\mathrm{NO}$ & & $\begin{array}{l}\text { Non- } \\
\text { sensitizer }\end{array}$ & & & & & REACH (2008-2014) & \\
\hline $\begin{array}{l}\mathrm{N}-\{[(1,1- \\
\text { dimethylethyl)oxy]carb } \\
\text { onyl\}-4-fluoro- } \beta-(4-\end{array}$ & $\begin{array}{l}\mathrm{CC}(\mathrm{C})(\mathrm{C}) \mathrm{OC}(=\mathrm{O}) \mathrm{NC}(\mathrm{C}(\mathrm{c} \\
1 \mathrm{ccc}(\mathrm{F}) \mathrm{cc} 1) \mathrm{c} 1 \mathrm{ccc}(\mathrm{F}) \mathrm{cc} 1 \\
\mathrm{C}(\mathrm{O})=0\end{array}$ & & $\begin{array}{l}\text { Non- } \\
\text { sensitizer }\end{array}$ & & & & & ICCVAM (2013) & \\
\hline
\end{tabular}




\begin{tabular}{|c|c|c|c|c|c|c|c|c|c|}
\hline $\begin{array}{l}\text { fluorophenyl)-I- } \\
\text { phenylalanine }\end{array}$ & & & & & & & & & \\
\hline $\begin{array}{l}\text { N1-[3-(1H-imidazol-1- } \\
\text { yl)propyl]-3- } \\
\text { methylbenzene-1,4- } \\
\text { diamine }\end{array}$ & $\begin{array}{l}\operatorname{Cc} 1 \mathrm{cc}(\mathrm{NCCCn} 2 \mathrm{ccnc} 2) \mathrm{cc} \\
\mathrm{c} 1 \mathrm{~N}\end{array}$ & & $\begin{array}{l}\text { Non- } \\
\text { sensitizer }\end{array}$ & & & & & REACH (2008-2014) & \\
\hline $\begin{array}{l}\mathrm{N} 2-(4,6-\text { diamino-1,3,5- } \\
\text { triazin-2-yl)-1,3,5- } \\
\text { triazine-2,4,6-triamine }\end{array}$ & $\begin{array}{l}\text { Nc1nc(N)nc(Nc2nc(N)n } \\
c(N) n 2) n 1\end{array}$ & & $\begin{array}{l}\text { Non- } \\
\text { sensitizer }\end{array}$ & & & & & REACH (2008-2014) & \\
\hline Nitrobenzene & $\mathrm{O}=\mathrm{N}(=\mathrm{O}) \mathrm{c} 1 \mathrm{ccccc} 1$ & & $\begin{array}{l}\text { Non- } \\
\text { sensitizer }\end{array}$ & & & & & REACH (2008-2014) & \\
\hline $\begin{array}{l}\text { O-propan-2-yl } \\
\text { ethylaminomethanethi } \\
\text { oate }\end{array}$ & $\mathrm{CCNC}(=\mathrm{S}) \mathrm{OC}(\mathrm{C}) \mathrm{C}$ & & $\begin{array}{l}\text { Non- } \\
\text { sensitizer }\end{array}$ & & & & & REACH (2008-2014) & \\
\hline Compound name & SMILES & $\begin{array}{l}\text { Human } \\
\text { result }\end{array}$ & $\begin{array}{l}\text { LLNA } \\
\text { result }\end{array}$ & DPRA & KeratinoSens & h-CLAT & $\begin{array}{l}\text { Human } \\
\text { reference }\end{array}$ & LLNA reference & Non-animal reference \\
\hline Octane-1,2-diol & $\operatorname{ccccccc}(0) \operatorname{co}$ & & $\begin{array}{l}\text { Non- } \\
\text { sensitizer }\end{array}$ & & & & & REACH (2008-2014) & \\
\hline Oxolane & C1Ccoc1 & & $\begin{array}{l}\text { Non- } \\
\text { sensitizer }\end{array}$ & & & & & REACH (2008-2014) & \\
\hline $\begin{array}{l}\text { Pentaerythritol ester of } \\
\text { wood resin }\end{array}$ & $\begin{array}{l}\mathrm{CC}(\mathrm{C}) \mathrm{C} 1=\mathrm{CC} 2=\mathrm{CCC} 3 \mathrm{C}(\mathrm{C} \\
)(\mathrm{CCCC} 33 \mathrm{C}(=0) \mathrm{OCC} 45 \mathrm{C} \\
\mathrm{C}(\mathrm{C} 4) \mathrm{C} 5) \mathrm{C} 2 \mathrm{CC} 1\end{array}$ & & $\begin{array}{l}\text { Non- } \\
\text { sensitizer }\end{array}$ & & & & & REACH (2008-2014) & \\
\hline Pentane-2,4-dione & $\mathrm{CC}(\mathrm{O})=\mathrm{CC}(\mathrm{C})=\mathrm{O}$ & & $\begin{array}{l}\text { Non- } \\
\text { sensitizer }\end{array}$ & & & & & REACH (2008-2014) & \\
\hline Pentyl propanoate & $\operatorname{cccCCOC}(=0) C C$ & & $\begin{array}{l}\text { Non- } \\
\text { sensitizer }\end{array}$ & & & & & REACH (2008-2014) & \\
\hline Phenethyl alcohol & OCCc1ccccc1 & & $\begin{array}{l}\text { Non- } \\
\text { sensitizer }\end{array}$ & & & & & $\begin{array}{l}\text { ICCVAM (2013) + } \\
\text { REACH (2008-2014) }\end{array}$ & \\
\hline $\begin{array}{l}\text { Phenylmethyl 2-(4- } \\
\text { fluoro-2-methylphenyl)- } \\
\text { 4-oxo-3,4-dihydro- } \\
\text { 1(2h)-pyridine- } \\
\text { carboxylate }\end{array}$ & $\begin{array}{l}\operatorname{Cc} 1 \mathrm{cc}(\mathrm{F}) \operatorname{ccc} 1 \mathrm{C} 1 \mathrm{CC}(=0) \\
\mathrm{C}=\mathrm{CN} 1 \mathrm{C}(=0) 0 \mathrm{Cc} 1 \mathrm{ccccc} \\
1\end{array}$ & & $\begin{array}{l}\text { Non- } \\
\text { sensitizer }\end{array}$ & & & & & ICCVAM (2013) & \\
\hline Pigment Violet 29 & $\begin{array}{l}\mathrm{O}=\mathrm{C} 1 \mathrm{NC}(=0) \mathrm{c} 2 \mathrm{ccc} 3 \mathrm{c} 4 \mathrm{c} \\
\mathrm{cc5}(=0) \mathrm{NC}(=0) \mathrm{c} 6 \mathrm{ccc}( \\
\mathrm{c7} \operatorname{ccc} 1 \mathrm{c} 2 \mathrm{c} 37) \mathrm{c} 4 \mathrm{c} 56\end{array}$ & & $\begin{array}{l}\text { Non- } \\
\text { sensitizer }\end{array}$ & & & & & REACH (2008-2014) & \\
\hline $\begin{array}{l}\text { Piperidine-1- } \\
\text { carbothioylsulfanyldisul } \\
\text { fanyldisulfanyl } \\
\text { piperidine-1- } \\
\text { carbodithioate } \\
\end{array}$ & $\begin{array}{l}\mathrm{S}=\mathrm{C}(\mathrm{SSSSSSC}(=\mathrm{S}) \mathrm{N} 1 \mathrm{CC} \\
\mathrm{CCC} 1) \mathrm{N} 1 \mathrm{CCCCC1}\end{array}$ & & $\begin{array}{l}\text { Non- } \\
\text { sensitizer }\end{array}$ & & & & & REACH (2008-2014) & \\
\hline Propanal & $\mathrm{CCC}=\mathrm{O}$ & & $\begin{array}{l}\text { Non- } \\
\text { sensitizer }\end{array}$ & & & & & REACH (2008-2014) & \\
\hline Quinacridone & $\begin{array}{l}\mathrm{O}=\mathrm{C} 1 \mathrm{c} 2 \mathrm{ccccc} 2 \mathrm{Nc} 2 \mathrm{cc} 3 \mathrm{C} \\
(=0) \mathrm{c} 4 \mathrm{ccccc} 4 \mathrm{Nc} 3 \mathrm{cc} 12\end{array}$ & & $\begin{array}{l}\text { Non- } \\
\text { sensitizer }\end{array}$ & & & & & REACH (2008-2014) & \\
\hline
\end{tabular}




\begin{tabular}{|c|c|c|c|c|c|c|c|c|c|}
\hline Quinoxyfen & $\begin{array}{l}\text { Fc1ccc(Oc2 } \mathrm{ccnc} 3 \mathrm{cc}(\mathrm{Cl}) \\
\mathrm{cc}(\mathrm{Cl}) \mathrm{c23}) \mathrm{cc} 1\end{array}$ & & $\begin{array}{l}\text { Non- } \\
\text { sensitizer }\end{array}$ & & & & & ICCVAM (2013) & \\
\hline Retapamulin & $\begin{array}{l}\mathrm{CC} 1 \mathrm{CCC} 23 \mathrm{CCC}(=0) \mathrm{C} 2 \mathrm{C} \\
1(\mathrm{C}) \mathrm{C}(\mathrm{CC}(\mathrm{C})(\mathrm{C}=\mathrm{C}) \mathrm{C}(\mathrm{O}) \mathrm{C} \\
3 \mathrm{C}) \mathrm{OC}(=\mathrm{O}) \mathrm{CSC} 1 \mathrm{CC} 2 \mathrm{CC} \\
\mathrm{C}(\mathrm{C} 1) \mathrm{N} 2 \mathrm{C}\end{array}$ & & $\begin{array}{l}\text { Non- } \\
\text { sensitizer }\end{array}$ & & & & & ICCVAM (2013) & \\
\hline Rifamycin & $\begin{array}{l}\mathrm{COC} 1 \mathrm{C}=\mathrm{COC} 2(\mathrm{C}) \mathrm{Oc} 3 \mathrm{c}( \\
\mathrm{C} 2=0) \mathrm{c} 2 \mathrm{c}(\mathrm{O}) \mathrm{cc}(\mathrm{NC}=\mathrm{O}) \\
\mathrm{C}(\mathrm{C})=\mathrm{CC}=\mathrm{CC}(\mathrm{C}) \mathrm{C}(\mathrm{O}) \mathrm{C}(\mathrm{C} \\
) \mathrm{C}(\mathrm{O}) \mathrm{C}(\mathrm{C}) \mathrm{C}(\mathrm{OC}(\mathrm{C})=\mathrm{O}) \mathrm{C} \\
1 \mathrm{C}) \mathrm{c}(\mathrm{O}) \mathrm{c} 2 \mathrm{c}(\mathrm{O}) \mathrm{c} 3 \mathrm{C}\end{array}$ & & $\begin{array}{l}\text { Non- } \\
\text { sensitizer }\end{array}$ & & & & & ICCVAM (2013) & \\
\hline $\begin{array}{l}\text { Sodium (2R)-2-[(2R)- } \\
4,5 \text {-dihydroxy-3- } \\
\text { oxofuran-2-yl]-2- } \\
\text { hydroxyethanolate }\end{array}$ & $\begin{array}{l}\mathrm{OCC}(0) \mathrm{C} 1 \mathrm{OC}(=0) \mathrm{C}(0)= \\
\mathrm{C} 10\end{array}$ & & $\begin{array}{l}\text { Non- } \\
\text { sensitizer }\end{array}$ & & & & & REACH (2008-2014) & \\
\hline Compound name & SMILES & $\begin{array}{l}\text { Human } \\
\text { result }\end{array}$ & $\begin{array}{l}\text { LLNA } \\
\text { result }\end{array}$ & DPRA & KeratinoSens & h-CLAT & $\begin{array}{l}\text { Human } \\
\text { reference }\end{array}$ & LLNA reference & Non-animal reference \\
\hline Sodium 2-chloroacetate & $\mathrm{OC}(=\mathrm{O}) \mathrm{CCl}$ & & $\begin{array}{l}\text { Non- } \\
\text { sensitizer }\end{array}$ & & & & & REACH (2008-2014) & \\
\hline $\begin{array}{l}\text { Sodium 2- } \\
\text { hydroxyethanesulfonat } \\
\text { e }\end{array}$ & $\operatorname{OCCS}(0)(=0)=0$ & & $\begin{array}{l}\text { Non- } \\
\text { sensitizer }\end{array}$ & & & & & REACH (2008-2014) & \\
\hline Sodium Thiocyanate & SC\#N & & $\begin{array}{l}\text { Non- } \\
\text { sensitizer }\end{array}$ & & & & & REACH (2008-2014) & \\
\hline Sodium octyl sulfate & $\begin{array}{l}\operatorname{cccccccc} 0 \operatorname{sos}(0)(=0)= \\
0\end{array}$ & & $\begin{array}{l}\text { Non- } \\
\text { sensitizer }\end{array}$ & & & & & REACH (2008-2014) & \\
\hline Succinic acid & $\mathrm{OC}(=0) \mathrm{CCC}(0)=0$ & & $\begin{array}{l}\text { Non- } \\
\text { sensitizer }\end{array}$ & & & & & ICCVAM (2013) & \\
\hline Yellow E-JD 3442 & $\begin{array}{l}\mathrm{CCC}(\mathrm{O}) \operatorname{COc} 1 \operatorname{ccc}(\mathrm{cc} 1) \mathrm{N}= \\
\mathrm{Nc} 1 \mathrm{ccc}(\mathrm{N}=\mathrm{Nc} 2 \operatorname{ccc}(\mathrm{c} 2) \\
\mathrm{S}(\mathrm{O})(=\mathrm{O})=\mathrm{O}) \mathrm{c}(\mathrm{NC}(\mathrm{C})=\mathrm{O} \\
\mathrm{c} 1\end{array}$ & & $\begin{array}{l}\text { Non- } \\
\text { sensitizer }\end{array}$ & & & & & ICCVAM (2013) & \\
\hline $\begin{array}{l}\text { [(1S,2R,5S)-5-methyl-2- } \\
\text { propan-2-ylcyclohexyl] } \\
\text { acetate }\end{array}$ & $\begin{array}{l}\mathrm{CC}(\mathrm{C}) \mathrm{C} 1 \mathrm{CCC}(\mathrm{C}) \mathrm{CC} 10 \mathrm{C}( \\
\mathrm{C})=0\end{array}$ & & $\begin{array}{l}\text { Non- } \\
\text { sensitizer }\end{array}$ & & & & & REACH (2008-2014) & \\
\hline $\begin{array}{l}\text { [2-[2,2-bis(prop-2- } \\
\text { enoyloxymethyl)butoxy } \\
\text { methyl]-2-(prop-2- } \\
\text { enoyloxymethyl)butyl] } \\
\text { prop-2-enoate }\end{array}$ & $\begin{array}{l}\mathrm{CCC}(\operatorname{COCC}(\mathrm{CC})(\mathrm{COC}(=0 \\
) \mathrm{C}=\mathrm{C}) \operatorname{coc}(=\mathrm{O}) \mathrm{C}=\mathrm{C})(\mathrm{CO} \\
\mathrm{C}(=\mathrm{O}) \mathrm{C}=\mathrm{C}) \operatorname{coc}(=0) \mathrm{C}=\mathrm{C}\end{array}$ & & $\begin{array}{l}\text { Non- } \\
\text { sensitizer }\end{array}$ & & & & & REACH (2008-2014) & \\
\hline $\begin{array}{l}\text { [2-amino-3-(4- } \\
\text { ethoxyphenyl)propyl](2 } \\
\text {-aminoethyl)amine }\end{array}$ & $\begin{array}{l}\mathrm{CCOc} 1 \mathrm{ccc}(\mathrm{CC}(\mathrm{N}) \mathrm{CNCC} \\
\mathrm{N}) \mathrm{cc} 1\end{array}$ & & $\begin{array}{l}\text { Non- } \\
\text { sensitizer }\end{array}$ & & & & & REACH (2008-2014) & \\
\hline $\begin{array}{l}\text { [4-(ethoxymethyl)-2,6- } \\
\text { dimethoxyphenyl]- } \\
\text { boronic acid }\end{array}$ & $\begin{array}{l}\mathrm{cCOCc} 1 \mathrm{cc}(\mathrm{OC}) \mathrm{c}(\mathrm{B}(\mathrm{O}) \mathrm{O}) \\
\mathrm{c}(\mathrm{OC}) \mathrm{c} 1\end{array}$ & & $\begin{array}{l}\text { Non- } \\
\text { sensitizer }\end{array}$ & & & & & ICCVAM (2013) & \\
\hline
\end{tabular}




\begin{tabular}{|c|c|c|c|c|c|c|c|c|c|}
\hline $\begin{array}{l}\text { [4S-[1(E),4a,5 } \alpha]]-1-[3- \\
\text { [2-[4-Methoxy-2- } \\
\text { (phenylmethoxy)- } \\
\text { benzoyl]-4- } \\
\text { propoxyphenyl]-1-oxo- } \\
\text { 2-propenyl]-3,4- } \\
\text { dimethyl-5-phenyl-2- } \\
\text { imidazoli-dinone }\end{array}$ & $\begin{array}{l}\mathrm{CCCOc} 1 \mathrm{ccc}(\mathrm{C}=\mathrm{CC}(=\mathrm{O}) \mathrm{N} \\
2 \mathrm{C}(\mathrm{C}(\mathrm{C}) \mathrm{N}(\mathrm{C}) \mathrm{C} 2=\mathrm{O}) \mathrm{c} 2 \mathrm{cc} \\
\operatorname{ccc} 2) \mathrm{c}(\mathrm{c} 1) \mathrm{C}(=\mathrm{O}) \mathrm{c} 1 \mathrm{ccc}( \\
\mathrm{OC}) \mathrm{cc} 10 \mathrm{Cc} 1 \mathrm{ccccc} 1\end{array}$ & & $\begin{array}{l}\text { Non- } \\
\text { sensitizer }\end{array}$ & & & & & ICCVAM (2013) & \\
\hline $\begin{array}{l}\text { acetic acid; } 2 \text {-(bis(2- } \\
\text { hydroxyethyl)amino)et } \\
\text { hanol }\end{array}$ & OCCN(CCO)CCO & & $\begin{array}{l}\text { Non- } \\
\text { sensitizer }\end{array}$ & & & & & REACH (2008-2014) & \\
\hline $\begin{array}{l}\text { azanium carbamate } \\
\text { hydrate }\end{array}$ & $N C(O)=O$ & & $\begin{array}{l}\text { Non- } \\
\text { sensitizer }\end{array}$ & & & & & REACH (2008-2014) & \\
\hline $\begin{array}{l}\text { benzyl- } \\
\text { trimethylazanium } \\
\text { chloride }\end{array}$ & $\mathrm{C}[\mathrm{N}+](\mathrm{C})(\mathrm{C}) \mathrm{Cc} 1 \mathrm{ccccc} 1$ & & $\begin{array}{l}\text { Non- } \\
\text { sensitizer }\end{array}$ & & & & & REACH (2008-2014) & \\
\hline Compound name & SMILES & $\begin{array}{l}\text { Human } \\
\text { result }\end{array}$ & $\begin{array}{l}\text { LLNA } \\
\text { result }\end{array}$ & DPRA & KeratinoSens & h-CLAT & $\begin{array}{l}\text { Human } \\
\text { reference }\end{array}$ & LLNA reference & Non-animal reference \\
\hline $\begin{array}{l}\text { bis(2-butoxyethyl) } \\
\text { hexanedioate }\end{array}$ & $\begin{array}{l}\text { CCCCOCCOC }(=0) C C C C \\
C(=0) \text { OCCOCCCC }\end{array}$ & & $\begin{array}{l}\text { Non- } \\
\text { sensitizer }\end{array}$ & & & & & REACH (2008-2014) & \\
\hline $\begin{array}{l}\text { bis(2-ethylhexyl) } \\
\text { butanedioate }\end{array}$ & $\begin{array}{l}\mathrm{CCCCC}(\mathrm{CC}) \mathrm{COC}(=0) \mathrm{CC} \\
\mathrm{C}(=0) \mathrm{OCC}(\mathrm{CC}) \mathrm{CCCC}\end{array}$ & & $\begin{array}{l}\text { Non- } \\
\text { sensitizer }\end{array}$ & & & & & REACH (2008-2014) & \\
\hline $\begin{array}{l}\text { bis-1,3-(2',5'- } \\
\text { dimethylphenyl)- } \\
\text { propane-1,3-dione }\end{array}$ & $\begin{array}{l}\operatorname{Cc} 1 \operatorname{ccc}(C) c(c 1) C(O)=C C \\
(=0) \operatorname{c1cc}(C) \operatorname{ccc} 1 C\end{array}$ & & $\begin{array}{l}\text { Non- } \\
\text { sensitizer }\end{array}$ & & & & & ICCVAM (2013) & \\
\hline but-2-enoic acid & $\mathrm{CC}=\mathrm{CC}(\mathrm{O})=\mathrm{O}$ & & $\begin{array}{l}\text { Non- } \\
\text { sensitizer }\end{array}$ & & & & & REACH (2008-2014) & \\
\hline $\begin{array}{l}\text { butyl 2-[(2-butoxy-2- } \\
\text { oxoethyl)sulfanylmethyl } \\
\text { sulfanyl]]acetate }\end{array}$ & $\begin{array}{l}\operatorname{ccccOC}(=0) \operatorname{cscscC}(= \\
\text { O) OCCCC }\end{array}$ & & $\begin{array}{l}\text { Non- } \\
\text { sensitizer }\end{array}$ & & & & & REACH (2008-2014) & \\
\hline $\begin{array}{l}\text { cis-4-Cyano-4-[3- } \\
\text { (cyclopentyloxy)-4- } \\
\text { methoxyphenyl]cyclo- } \\
\text { hexanecarboxylic acid }\end{array}$ & $\begin{array}{l}\operatorname{cOc} 1 \mathrm{ccc}(\mathrm{cc} 10 \mathrm{C} 1 \mathrm{CCCC} 1 \\
) \mathrm{C1}(\mathrm{CCC}(\mathrm{CC} 1) \mathrm{C}(\mathrm{O})=0) \mathrm{C} \\
\# \mathrm{~N}\end{array}$ & & $\begin{array}{l}\text { Non- } \\
\text { sensitizer }\end{array}$ & & & & & ICCVAM (2013) & \\
\hline $\begin{array}{l}\text { decanoic acid; 2-ethyl- } \\
2- \\
\text { (hydroxymethyl)propan } \\
\text { e-1,3-diol; octanoic acid }\end{array}$ & $\operatorname{cccccccccc}(0)=0$ & & $\begin{array}{l}\text { Non- } \\
\text { sensitizer }\end{array}$ & & & & & REACH (2008-2014) & \\
\hline $\begin{array}{l}\text { decyl (Z)-octadec-9- } \\
\text { enoate }\end{array}$ & $\begin{array}{l}\text { CCCCCCCCCCOC(=O)CC } \\
\text { CCCCCC }=\text { CCCCCCCCC }\end{array}$ & & $\begin{array}{l}\text { Non- } \\
\text { sensitizer }\end{array}$ & & & & & REACH (2008-2014) & \\
\hline $\begin{array}{l}\text { dibutylcarbamothioylsu } \\
\text { Ifanylmethyl } \\
\text { (dibutylamino)methane } \\
\text { dithioate }\end{array}$ & $\begin{array}{l}\text { CCCCN }(C C C C) C(=S) S C S \\
C(=S) N(C C C C) C C C C\end{array}$ & & $\begin{array}{l}\text { Non- } \\
\text { sensitizer }\end{array}$ & & & & & REACH (2008-2014) & \\
\hline $\begin{array}{l}\text { didodecyl (E)-but-2- } \\
\text { enedioate }\end{array}$ & $\begin{array}{l}\text { CCCCCCCCCCCCOC( }=0) \\
\mathrm{C}=\mathrm{CC}(=0) \text { OccccccccC } \\
\mathrm{CCC}\end{array}$ & & $\begin{array}{l}\text { Non- } \\
\text { sensitizer }\end{array}$ & & & & & REACH (2008-2014) & \\
\hline
\end{tabular}




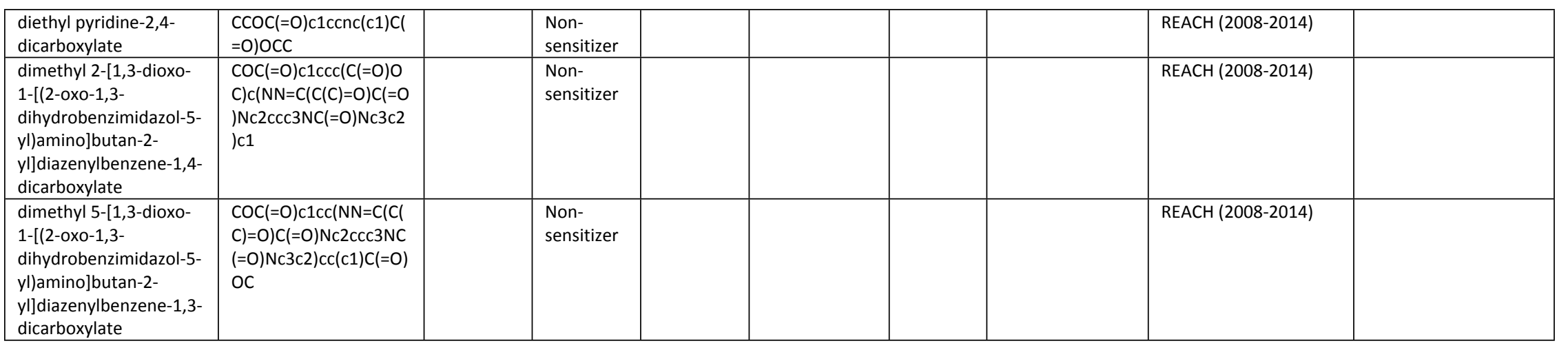

\begin{tabular}{|c|c|c|c|c|c|c|c|c|c|}
\hline Compound name & SMILES & $\begin{array}{l}\text { Human } \\
\text { result }\end{array}$ & $\begin{array}{l}\text { LLNA } \\
\text { result }\end{array}$ & DPRA & KeratinoSens & h-CLAT & $\begin{array}{l}\text { Human } \\
\text { reference }\end{array}$ & LLNA reference & Non-animal reference \\
\hline $\begin{array}{l}\text { disodium 3-methyl-5- } \\
\text { (3-methyl-5- } \\
\text { sulfonatophenoxy)benz } \\
\text { enesulfonate }\end{array}$ & $\begin{array}{l}\operatorname{cc1} 1 \mathrm{cc}(\mathrm{Oc} 2 \mathrm{cc}(\mathrm{C}) \mathrm{cc}(\mathrm{c} 2) \mathrm{S}( \\
\mathrm{O})(=0)=0) \mathrm{cc}(\mathrm{c} 1) \mathrm{s}(\mathrm{O})(= \\
\mathrm{O})=0\end{array}$ & & $\begin{array}{l}\text { Non- } \\
\text { sensitizer }\end{array}$ & & & & & REACH (2008-2014) & \\
\hline $\begin{array}{l}\text { ethyl (2S)-2- } \\
\text { hydroxypropanoate }\end{array}$ & $\operatorname{ccOC}(=0) C(C) O$ & & $\begin{array}{l}\text { Non- } \\
\text { sensitizer }\end{array}$ & & & & & REACH (2008-2014) & \\
\hline $\begin{array}{l}\text { ethyl 1-(4- } \\
\text { methoxyphenyl)-7-oxo- } \\
\text { 6-[4-(2-oxopiperidin-1- } \\
\text { yl)phenyl]- } \\
1 \mathrm{H}, 4 \mathrm{H}, 5 \mathrm{H}, 6 \mathrm{H}, 7 \mathrm{H} \text { - } \\
\text { pyrazolo[3,4-c]pyridine- } \\
\text { 3-carboxylate }\end{array}$ & $\begin{array}{l}\mathrm{CCOC}(=0) \mathrm{c} 1 \mathrm{nn}(\mathrm{c} 2 \mathrm{c} 1 \mathrm{CC} \\
\mathrm{N}(\mathrm{C2}=\mathrm{O}) \mathrm{c} 1 \mathrm{ccc}(\mathrm{cc} 1) \mathrm{N} 1 \mathrm{C} \\
\mathrm{CCCC} 1=0)- \\
\mathrm{c} 1 \mathrm{ccc}(\mathrm{OC}) \mathrm{cc} 1\end{array}$ & & $\begin{array}{l}\text { Non- } \\
\text { sensitizer }\end{array}$ & & & & & REACH (2008-2014) & \\
\hline $\begin{array}{l}\text { ethyl } 2,2,2- \\
\text { trifluoroacetate }\end{array}$ & $\mathrm{CCOC}(=0) \mathrm{C}(\mathrm{F})(\mathrm{F}) \mathrm{F}$ & & $\begin{array}{l}\text { Non- } \\
\text { sensitizer }\end{array}$ & & & & & REACH (2008-2014) & \\
\hline $\begin{array}{l}\text { ethyl 2- } \\
\text { diethoxyphosphorylace } \\
\text { tate }\end{array}$ & $\begin{array}{l}\mathrm{CCOC}(=\mathrm{O}) \mathrm{CP}(=\mathrm{O})(\mathrm{OCC}) \\
\mathrm{OCC}\end{array}$ & & $\begin{array}{l}\text { Non- } \\
\text { sensitizer }\end{array}$ & & & & & REACH (2008-2014) & \\
\hline $\begin{array}{l}\text { ethyl 4-[2-chloro-4-[3- } \\
\text { chloro-4-[(3- } \\
\text { ethoxycarbonyl-5-oxo- } \\
\text { 1-phenyl-4H-pyrazol-4- } \\
\text { yl)diazenyl]phenyl]phen } \\
\text { yl]diazenyl-5-oxo-1- } \\
\text { phenyl-4H-pyrazole-3- } \\
\text { carboxylate } \\
\end{array}$ & $\begin{array}{l}\mathrm{CCOC}(=\mathrm{O}) \mathrm{C} 1=\mathrm{C}(\mathrm{N}=\mathrm{Nc} 2 \\
\operatorname{ccc}(\mathrm{cc} 2 \mathrm{Cl})- \\
\operatorname{c2ccc}(\mathrm{N}=\mathrm{NC} 3=\mathrm{C}(\mathrm{NN}(\mathrm{C} 3 \\
=\mathrm{O}) \mathrm{c} 3 \mathrm{ccccc} 3) \mathrm{C}(=\mathrm{O}) \mathrm{OCC} \\
) \mathrm{c}(\mathrm{Cl}) \mathrm{c} 2) \mathrm{C}(=\mathrm{O}) \mathrm{N}(\mathrm{N} 1) \mathrm{c} 1 \mathrm{c} \\
\operatorname{cccc} 1\end{array}$ & & $\begin{array}{l}\text { Non- } \\
\text { sensitizer }\end{array}$ & & & & & REACH (2008-2014) & \\
\hline imidazolidin-2-one & $\mathrm{O}=\mathrm{C} 1 \mathrm{NCCN} 1$ & & $\begin{array}{l}\text { Non- } \\
\text { sensitizer }\end{array}$ & & & & & REACH (2008-2014) & \\
\hline
\end{tabular}




\begin{tabular}{|c|c|c|c|c|c|c|c|c|c|}
\hline $\begin{array}{l}\text { lithium 12- } \\
\text { hydroxyoctadecanoate }\end{array}$ & $\begin{array}{l}\text { CCCCCCC(O)CCCCCCCC } \\
\operatorname{ccc}(0)=0\end{array}$ & & $\begin{array}{l}\text { Non- } \\
\text { sensitizer }\end{array}$ & & & & & REACH (2008-2014) & \\
\hline $\begin{array}{l}\text { methyl 4- } \\
\text { (dimethylcarbamoyl)-2- } \\
\text { methylbutanoate }\end{array}$ & $\begin{array}{l}\operatorname{COC}(=O) C(C) C C C(=0) N \\
\text { (C)C }\end{array}$ & & $\begin{array}{l}\text { Non- } \\
\text { sensitizer }\end{array}$ & & & & & REACH (2008-2014) & \\
\hline $\begin{array}{l}\text { methyl 4-[(2,5- } \\
\text { dichlorophenyl)carbam } \\
\text { oyl]-2-[(2Z)-2-[3-[(2- } \\
\text { methoxyphenyl)carbam } \\
\text { oyl]-2-oxonaphthalen- } \\
1- \\
\text { ylidene]hydrazinyl]benz } \\
\text { oate }\end{array}$ & $\begin{array}{l}\operatorname{COC}(=0) \operatorname{c1ccc}(\operatorname{cc} 1 \mathrm{~N}=\mathrm{N} \\
\mathrm{c} 1 \mathrm{c}(\mathrm{O}) \mathrm{c}(\operatorname{cc} 2 \operatorname{ccccc} 12) \mathrm{Cl} \\
=0) \mathrm{Nc} 1 \mathrm{ccccc} 1 \mathrm{OC}) \mathrm{C}(=\mathrm{O} \\
) \mathrm{Nc1} 1 \mathrm{cc}(\mathrm{Cl}) \operatorname{ccc} 1 \mathrm{Cl}\end{array}$ & & $\begin{array}{l}\text { Non- } \\
\text { sensitizer }\end{array}$ & & & & & REACH (2008-2014) & \\
\hline methyl dec-9-enoate & $\operatorname{cOC}(=0) \operatorname{cccccccC}=C$ & & $\begin{array}{l}\text { Non- } \\
\text { sensitizer }\end{array}$ & & & & & REACH (2008-2014) & \\
\hline oxan-2-one & $\mathrm{O}=\mathrm{C} 1 \mathrm{CCCCO} 1$ & & $\begin{array}{l}\text { Non- } \\
\text { sensitizer }\end{array}$ & & & & & REACH (2008-2014) & \\
\hline Compound name & SMILES & $\begin{array}{l}\text { Human } \\
\text { result }\end{array}$ & $\begin{array}{l}\text { LLNA } \\
\text { result }\end{array}$ & DPRA & KeratinoSens & h-CLAT & $\begin{array}{l}\text { Human } \\
\text { reference }\end{array}$ & LLNA reference & Non-animal reference \\
\hline oxepan-2-one & $\mathrm{O}=\mathrm{C} 1 \mathrm{CCCCCO} 1$ & & $\begin{array}{l}\text { Non- } \\
\text { sensitizer }\end{array}$ & & & & & REACH (2008-2014) & \\
\hline oxiran-2-ylmethanol & OCC1CO1 & & $\begin{array}{l}\text { Non- } \\
\text { sensitizer }\end{array}$ & & & & & REACH (2008-2014) & \\
\hline oxolan-2-one & $\mathrm{O}=\mathrm{C} 1 \mathrm{CCCO} 1$ & & $\begin{array}{l}\text { Non- } \\
\text { sensitizer }\end{array}$ & & & & & REACH (2008-2014) & \\
\hline oxolan-2-ylmethanol & OCC1CCCO1 & & $\begin{array}{l}\text { Non- } \\
\text { sensitizer }\end{array}$ & & & & & REACH (2008-2014) & \\
\hline $\begin{array}{l}\text { potassium } \\
\text { (2R,3S,4R,5R)-2,3,4,5,6- } \\
\text { pentahydroxyhexanoat } \\
\text { e }\end{array}$ & $\begin{array}{l}\mathrm{OCC}(\mathrm{O}) \mathrm{C}(\mathrm{O}) \mathrm{C}(\mathrm{O}) \mathrm{C}(\mathrm{O}) \mathrm{C}( \\
\mathrm{O})=\mathrm{O}\end{array}$ & & $\begin{array}{l}\text { Non- } \\
\text { sensitizer }\end{array}$ & & & & & REACH (2008-2014) & \\
\hline $\begin{array}{l}\text { potassium } 2,2,2- \\
\text { trifluoroacetate }\end{array}$ & $\mathrm{OC}(=\mathrm{O}) \mathrm{C}(\mathrm{F})(\mathrm{F}) \mathrm{F}$ & & $\begin{array}{l}\text { Non- } \\
\text { sensitizer }\end{array}$ & & & & & REACH (2008-2014) & \\
\hline $\begin{array}{l}\text { potassium 6-methyl- } \\
\text { 2,2-dioxooxathiazin-4- } \\
\text { olate }\end{array}$ & $\begin{array}{l}\mathrm{CC1}=\mathrm{CC}(=0) \mathrm{NS}(=0)(=0 \\
\mathrm{O} 1\end{array}$ & & $\begin{array}{l}\text { Non- } \\
\text { sensitizer }\end{array}$ & & & & & REACH (2008-2014) & \\
\hline $\begin{array}{l}\text { potassium N- } \\
\text { carbamoylcarbamate }\end{array}$ & $N C(=0) N C(O)=0$ & & $\begin{array}{l}\text { Non- } \\
\text { sensitizer }\end{array}$ & & & & & REACH (2008-2014) & \\
\hline $\begin{array}{l}\text { propyl (2S)-2- } \\
\text { hydroxypropanoate }\end{array}$ & $\operatorname{CCCOC}(=0) C(C) O$ & & $\begin{array}{l}\text { Non- } \\
\text { sensitizer }\end{array}$ & & & & & REACH (2008-2014) & \\
\hline $\begin{array}{l}\text { sodium 2-hydroxy-3- } \\
\text { prop-2-enoxypropane- } \\
\text { 1-sulfonate }\end{array}$ & $\begin{array}{l}\mathrm{OC}(\mathrm{COCC}=\mathrm{C}) \mathrm{CS}(\mathrm{O})(=\mathrm{O}) \\
=\mathrm{O}\end{array}$ & & $\begin{array}{l}\text { Non- } \\
\text { sensitizer }\end{array}$ & & & & & REACH (2008-2014) & \\
\hline $\begin{array}{l}\text { sodium 3,5- } \\
\text { bis(methoxycarbonyl)b } \\
\text { enzenesulfonate }\end{array}$ & $\begin{array}{l}\operatorname{COC}(=0) \operatorname{c1cc}(\operatorname{cc}(\mathrm{c} 1) \mathrm{S}(\mathrm{O} \\
)(=0)=0) \mathrm{C}(=0) \mathrm{OC}\end{array}$ & & $\begin{array}{l}\text { Non- } \\
\text { sensitizer }\end{array}$ & & & & & REACH (2008-2014) & \\
\hline
\end{tabular}




\begin{tabular}{|c|c|c|c|c|c|c|c|c|c|}
\hline $\begin{array}{l}\text { tert-Butyl-3- } \\
\text { aminobenzoate }\end{array}$ & $\begin{array}{l}\mathrm{CC}(\mathrm{C})(\mathrm{C}) \mathrm{OC}(=0) \mathrm{c} 1 \mathrm{cccc}( \\
\mathrm{N}) \mathrm{c} 1\end{array}$ & & $\begin{array}{l}\text { Non- } \\
\text { sensitizer }\end{array}$ & & & & & ICCVAM (2013) & \\
\hline $\begin{array}{l}\text { tert-butyl N-(2-\{3- } \\
\text { cyano-2- } \\
\text { azabicyclo[3.1.0]hexan- } \\
\text { 2-yl\}-1-(3- } \\
\text { hydroxyadamantan-1- } \\
\text { yl)-2- } \\
\text { oxoethyl)carbamate }\end{array}$ & $\begin{array}{l}\mathrm{CC}(\mathrm{C})(\mathrm{C}) \mathrm{OC}(=\mathrm{O}) \mathrm{NC}(\mathrm{C}(= \\
\mathrm{O}) \mathrm{N} 1 \mathrm{C} 2 \mathrm{CC} 2 \mathrm{CC} 1 \mathrm{CHN}) \mathrm{C1} \\
2 \mathrm{CC} 3 \mathrm{CC}(\mathrm{CC}(\mathrm{O})(\mathrm{C} 3) \mathrm{C} 1) \mathrm{C} \\
2\end{array}$ & & $\begin{array}{l}\text { Non- } \\
\text { sensitizer }\end{array}$ & & & & & REACH (2008-2014) & \\
\hline $\begin{array}{l}\text { tetramethylazanium } \\
\text { chloride }\end{array}$ & $\mathrm{C}[\mathrm{N}+](\mathrm{C})(\mathrm{C}) \mathrm{C}$ & & $\begin{array}{l}\text { Non- } \\
\text { sensitizer }\end{array}$ & & & & & REACH (2008-2014) & \\
\hline $\begin{array}{l}\text { trans-2-Methyl-2- } \\
\text { butenal }\end{array}$ & $\mathrm{CC}=\mathrm{C}(\mathrm{C}) \mathrm{C}=\mathrm{O}$ & & $\begin{array}{l}\text { Non- } \\
\text { sensitizer }\end{array}$ & & & & & ICCVAM (2013) & \\
\hline $\begin{array}{l}\text { tricyclo[3.3.1.1.1,7]tetras } \\
\text { iloxane-1,3,5,7-tetrol }\end{array}$ & $\begin{array}{l}\mathrm{O}[\mathrm{Si}] 12 \mathrm{O}[\mathrm{Si}] 3(\mathrm{O}) \mathrm{O}[\mathrm{Si}]( \\
\mathrm{O})(\mathrm{O} 1) \mathrm{O}[\mathrm{Si}](\mathrm{O})(\mathrm{O} 2) \mathrm{O} 3\end{array}$ & & $\begin{array}{l}\text { Non- } \\
\text { sensitizer }\end{array}$ & & & & & REACH (2008-2014) & \\
\hline $\begin{array}{l}\text { triethoxy- } \\
\text { hydroxyphosphanium }\end{array}$ & $\operatorname{CCOP}(=0)(O C C) O C C$ & & $\begin{array}{l}\text { Non- } \\
\text { sensitizer }\end{array}$ & & & & & REACH (2008-2014) & \\
\hline Compound name & SMILES & $\begin{array}{l}\text { Human } \\
\text { result }\end{array}$ & $\begin{array}{l}\text { LLNA } \\
\text { result }\end{array}$ & DPRA & KeratinoSens & h-CLAT & $\begin{array}{l}\text { Human } \\
\text { reference }\end{array}$ & LLNA reference & Non-animal reference \\
\hline $\begin{array}{l}\text { trimethyl-[3-(2- } \\
\text { methylprop-2- } \\
\text { enoylamino)propyl]aza } \\
\text { nium chloride }\end{array}$ & $\begin{array}{l}\mathrm{CC}(=\mathrm{C}) \mathrm{C}(=\mathrm{O}) \mathrm{NCCC}[\mathrm{N}+]( \\
\text { C)(C)C }\end{array}$ & & $\begin{array}{l}\text { Non- } \\
\text { sensitizer }\end{array}$ & & & & & REACH (2008-2014) & \\
\hline $\begin{array}{l}\text { trimethyl-[3-(prop-2- } \\
\text { enoylamino)propyl]aza } \\
\text { nium chloride }\end{array}$ & $\begin{array}{l}\mathrm{C}[\mathrm{N}+](\mathrm{C})(\mathrm{C}) \mathrm{CCCNC}(=\mathrm{O}) \\
\mathrm{C}=\mathrm{C}\end{array}$ & & $\begin{array}{l}\text { Non- } \\
\text { sensitizer }\end{array}$ & & & & & REACH (2008-2014) & \\
\hline $\begin{array}{l}\text { tris(1-chloropropan-2- } \\
\text { yl) phosphate }\end{array}$ & $\begin{array}{l}\mathrm{CC}(\mathrm{CCl}) \mathrm{OP}(=0)(\mathrm{OC}(\mathrm{C}) \mathrm{C} \\
\mathrm{Cl}) \mathrm{OC}(\mathrm{C}) \mathrm{CCl}\end{array}$ & & $\begin{array}{l}\text { Non- } \\
\text { sensitizer }\end{array}$ & & & & & REACH (2008-2014) & \\
\hline $\begin{array}{l}\text { tris(4-nonylphenyl) } \\
\text { phosphite }\end{array}$ & 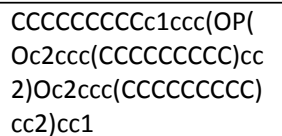 & & $\begin{array}{l}\text { Non- } \\
\text { sensitizer }\end{array}$ & & & & & REACH (2008-2014) & \\
\hline $\begin{array}{l}\text { tris(\{[1,1'-biphenyl]-4- } \\
\text { yl\})-1,3,5-triazine }\end{array}$ & $\begin{array}{l}\text { c1ccc(cc1)-c1ccc(cc1)- } \\
\text { c1nc(nc(n1)- } \\
\text { c1ccc(cc1)-c1ccccc1)- } \\
\text { c1ccc(cc1)-c1 } 1 \text { ccccc1 }\end{array}$ & & $\begin{array}{l}\text { Non- } \\
\text { sensitizer }\end{array}$ & & & & & REACH (2008-2014) & \\
\hline$\beta$-Terpinene & $\mathrm{CC}(\mathrm{C}) \mathrm{C} 1=\mathrm{CCC}(=\mathrm{C}) \mathrm{CC} 1$ & & $\begin{array}{l}\text { Non- } \\
\text { sensitizer }\end{array}$ & & & & & ICCVAM (2013) & \\
\hline $\begin{array}{l}\text { Tosylchloramide } \\
\text { sodium (Chloramine T) }\end{array}$ & $\begin{array}{l}\mathrm{CC} 1=\mathrm{CC}=\mathrm{C}(\mathrm{C}=\mathrm{C} 1) \mathrm{S}(=\mathrm{O})( \\
=0)[\mathrm{N}-] \mathrm{Cl} .[\mathrm{Na}+]\end{array}$ & & & Positive & Positive & Positive & & & Urbisch et al. (2015) \\
\hline $\begin{array}{l}2,4,6- \\
\text { Trinitrobenzenesulfonic } \\
\text { acid }\end{array}$ & $\begin{array}{l}\mathrm{O}=\mathrm{S}(=\mathrm{O})(\mathrm{O}) \mathrm{c} 1 \mathrm{c}(\mathrm{cc}(\mathrm{cc} 1[ \\
\mathrm{N}+]([\mathrm{O}-])=\mathrm{O})[\mathrm{N}+]([\mathrm{O}- \\
])=\mathrm{O})[\mathrm{N}+]([\mathrm{O}-])=\mathrm{O}\end{array}$ & & & Positive & Positive & & & & Urbisch et al. (2015) \\
\hline $\begin{array}{l}\text { 5-Chloro-2-methyl-4- } \\
\text { isothiazolin-3- } \\
\text { one (MCl) }\end{array}$ & $\operatorname{S1N}(C) C(=0) C=C 1(C l)$ & & & Positive & Positive & & & & Urbisch et al. (2015) \\
\hline
\end{tabular}




\begin{tabular}{|c|c|c|c|c|c|c|c|c|c|}
\hline Fumaric acid & $C(C=C C(=0) O)(O)=0$ & & & Positive & Negative & & & & Urbisch et al. (2015) \\
\hline Glucose & $\begin{array}{l}C(C(C(C(C(C O) O) O) O) O \\
)=0\end{array}$ & & & Positive & Negative & & & & Urbisch et al. (2015) \\
\hline $\begin{array}{l}\text { Isophorone } \\
\text { diisocyanate }\end{array}$ & $\begin{array}{l}\mathrm{CC1}(\mathrm{C}) \mathrm{CC}(\mathrm{N}=\mathrm{C}=0) \mathrm{CC}(\mathrm{C} \\
)(\mathrm{CN}=\mathrm{C}=\mathrm{O}) \mathrm{C1}\end{array}$ & & & Positive & & & & & Urbisch et al. (2015) \\
\hline Dimethyl formamide & $\mathrm{O}=\mathrm{CN}(\mathrm{C}) \mathrm{C}$ & & & Negative & Negative & Negative & & & Urbisch et al. (2015) \\
\hline $\begin{array}{l}\text { Carbonic acid, dioctyl } \\
\text { ester }\end{array}$ & $\begin{array}{l}\text { CCCCCCCCOC(=0)OCC } \\
\text { CCCCCC }\end{array}$ & & & Negative & & & & & Urbisch et al. (2015) \\
\hline $\begin{array}{l}\text { Cocamidopropyl } \\
\text { betaine }\end{array}$ & $\begin{array}{l}\text { [O- } \\
] \mathrm{C}(=\mathrm{O}) \mathrm{C}[\mathrm{N}+](\mathrm{CCCNC}= \\
\mathrm{O}) \mathrm{CCCCCCCCCCC})(\mathrm{C}) \mathrm{C}\end{array}$ & & & Negative & & & & & Urbisch et al. (2015) \\
\hline Dioctyl ether & $\begin{array}{l}\mathrm{C}(\mathrm{CC}) \mathrm{CCCCCOCCCCCCC} \\
\mathrm{C}\end{array}$ & & & Negative & & & & & Urbisch et al. (2015) \\
\hline $\begin{array}{l}\text { Hexadecyltrimethylam } \\
\text { monium bromide } \\
\text { (Cetrimide) }\end{array}$ & $\begin{array}{l}\mathrm{cCCCCCCCCCCCCCCCC[} \\
\mathrm{N}+](\mathrm{C})(\mathrm{C}) \mathrm{C} \text {. [Br-] }\end{array}$ & & & Negative & & & & & Urbisch et al. (2015) \\
\hline Compound name & SMILES & $\begin{array}{l}\text { Human } \\
\text { result }\end{array}$ & $\begin{array}{l}\text { LLNA } \\
\text { result }\end{array}$ & DPRA & KeratinoSens & h-CLAT & $\begin{array}{l}\text { Human } \\
\text { reference }\end{array}$ & LLNA reference & Non-animal reference \\
\hline $\begin{array}{l}\text { Octanoic acid, 4- } \\
\text { methyl-2-pentylbutyl } \\
\text { ester }\end{array}$ & $\begin{array}{l}\mathrm{O}=\mathrm{C}(\mathrm{OCC}(\mathrm{CCC}) \mathrm{CCCCC}) \\
\mathrm{CCCCCCC}\end{array}$ & & & Negative & & & & & Urbisch et al. (2015) \\
\hline 1-Benzoylacetone & $\mathrm{O}=\mathrm{C}(\mathrm{c} 1 \mathrm{ccccc} 1) \mathrm{CC}(=0) \mathrm{C}$ & & & & Positive & Positive & & & Urbisch et al. (2015) \\
\hline 1-Vinyl pyridine & $\mathrm{c} 1(\mathrm{C}=\mathrm{C}) \operatorname{ccccn} 1$ & & & & Positive & Positive & & & Urbisch et al. (2015) \\
\hline Dextran & $\begin{array}{l}\mathrm{C}(\mathrm{C} 1 \mathrm{C}(\mathrm{C}(\mathrm{C}(\mathrm{C}(\mathrm{O} 1) \mathrm{OCC2} \\
\mathrm{C}(\mathrm{C}(\mathrm{C}(\mathrm{C}(\mathrm{O}) \mathrm{OCC}(\mathrm{C}(\mathrm{C}(\mathrm{Cl}( \\
\mathrm{C}=0) \mathrm{O}) \mathrm{O}) \mathrm{O}) \mathrm{O}) \mathrm{O}) \mathrm{O}) \mathrm{O}) \\
\text { O)O)O)O }\end{array}$ & & & & Negative & Negative & & & Urbisch et al. (2015) \\
\hline
\end{tabular}

